\title{
From street-level to screen-level bureaucracy: Front-line public servants' recordkeeping behaviours in ICT-enabled agencies
}

\author{
By \\ Anita Jane Rapson
}

A thesis

submitted to the Victoria University of Wellington in fulfilment of the requirements for the degree of Doctor of Philosophy

Victoria University of Wellington 2018 


\section{Abstract}

Recordkeeping is a ubiquitous task within the public sector: public agencies rely on evidence of activities, in the form of public records, to coordinate activities, track resources, establish precedents, monitor performance as well as maintain consistency and continuity. Many public servants are responsible for recordkeeping; however, to date, little is known about the recordkeeping behaviours of these individuals, particularly about the recordkeeping behaviours of public servants who interact with members of the public on a daily basis.

The purpose of this study is to explore the recordkeeping behaviours of New Zealand front-line public servants within ICT-enabled state sector agencies to understand these behaviours and their associated governance implications. This inductive study began with multiple case studies that sought to explore the recordkeeping behaviours of front-line public servants. The second data collection phase involved a series of qualitative focus group interviews that explored the wider implications of the case studies, with the specific focus on understanding the governance implications. The empirical data was reviewed, drawing on the three theoretical concepts that established the analytical lens for this study: recordkeeping informatics, personal information management strategies, and the influencing factors on front-line public servants' decision-making behaviours.

Findings from this study suggest that front-line public servants have varying degrees of discretion to perform recordkeeping behaviours. A key outcome of this research is the identification of factors that influence the recordkeeping behaviours of front-line public servants: personal characteristics, organisational characteristics and the broader community. 


\section{Acknowledgements}

The writing of a doctoral thesis is not an easy task and one that cannot be completed without the efforts, expertise and support of many people.

I owe an immense debt of gratitude to my primary supervisor, Professor Miriam Lips, for her support, insightful feedback, patience and understanding throughout this long, long journey. I would also like to thank my secondary supervisors, Associate Professor Michael Macaulay and the early involvement of Dr Chris Eichbaum for their careful reading, assistance, ideas, and very constructive and thought-provoking feedback. I'd also like to extend my thanks to Tony Hooper who was instrumental in encouraging me to undertake postgraduate research.

I particularly wish to thank the volunteers and the organisations who supported me with their time, access and willingness to contribute to this research. Without them, and their involvement, this research would not have been possible.

This thesis has been a part-time undertaking with an extraordinarily long gestation, during which births, deaths, and everything in between have continued. As such, I offer special thanks to my parents for their continued support - and their many offers of childcare. Thanks to my children, Ada, Hazel, and our third daughter, who is soon to make her appearance, for their constant willingness to provide distractions. And finally, to Matt, for 'holding down the fort' and for encouraging me to work hard (maybe now he'll find time to read the final thesis).

While this thesis would not have been possible without the support and cooperation of many people, errors, omissions and weaknesses are mine entirely. 


\section{Table of Contents}

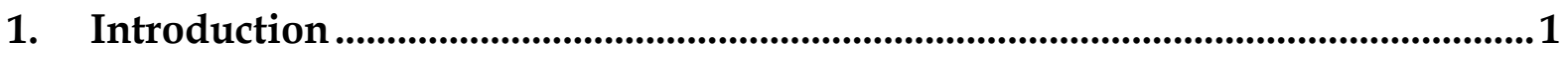

1.1 Introduction to this chapter ......................................................................................................1

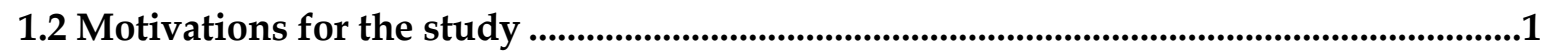

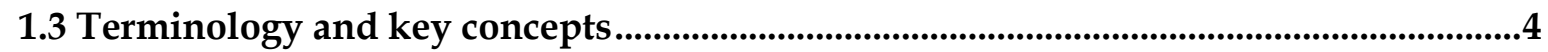

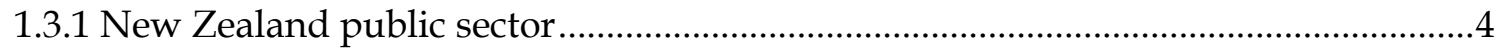

1.3.2 Information and Communication Technologies ............................................................

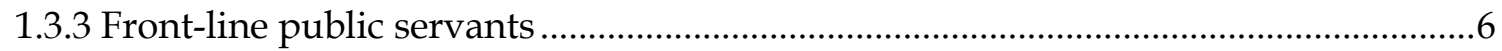

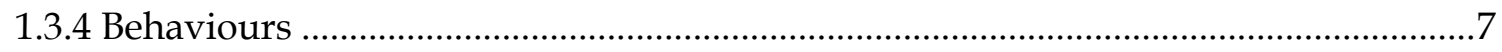

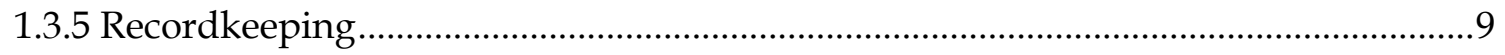

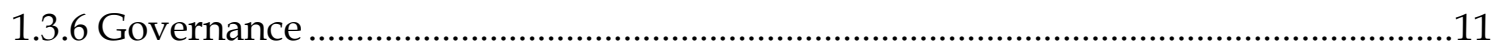

1.4 The New Zealand public sector context for recordkeeping ................................................11

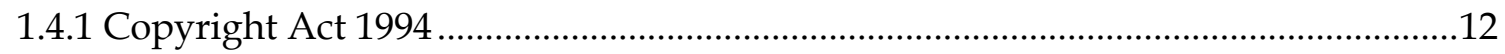

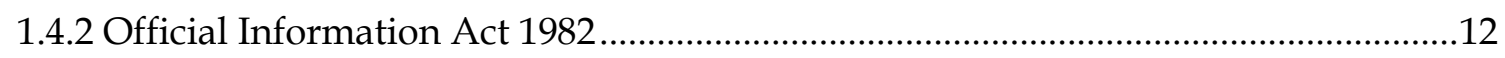

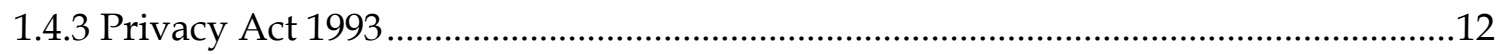

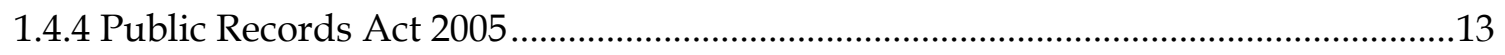

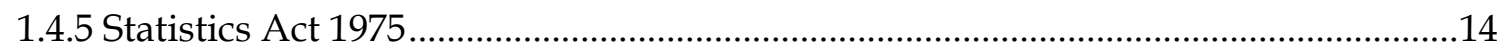

1.4.6 Contract and Commercial Law Act.................................................................................14

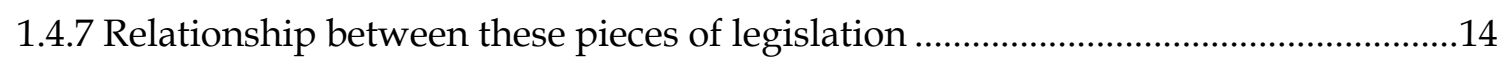

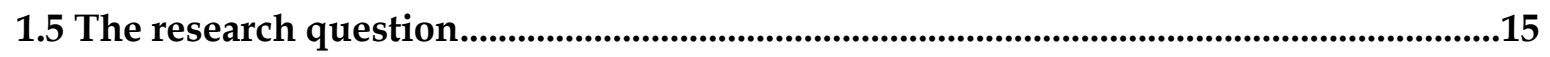

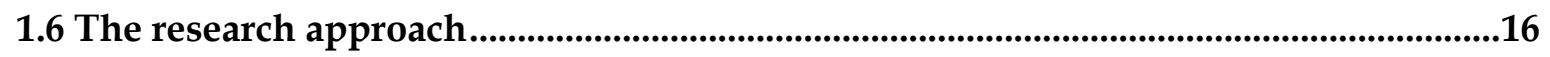

1.7 The significance of the study ......................................................................................................19

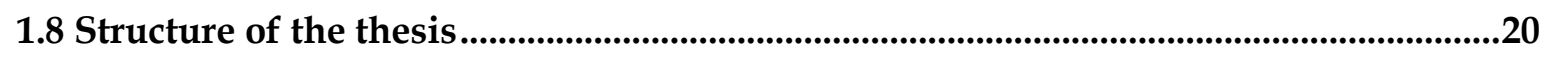

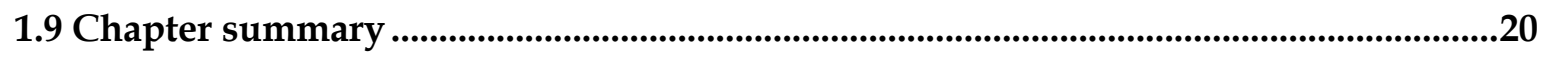

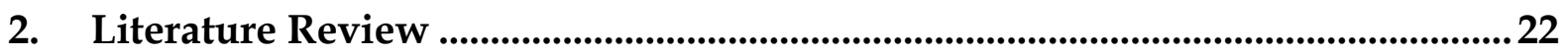

2.1 Introduction to this chapter ....................................................................................................22

2.2 Creating, capturing, organising and disseminating recorded information as

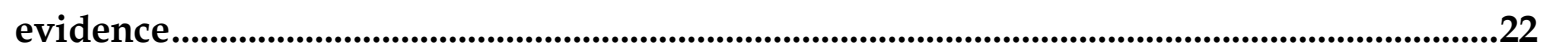

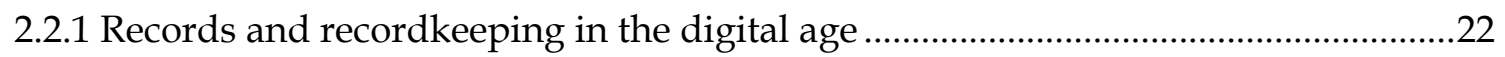

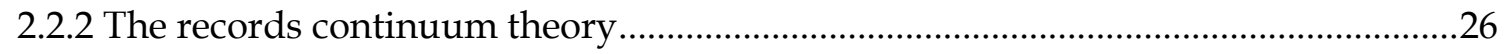

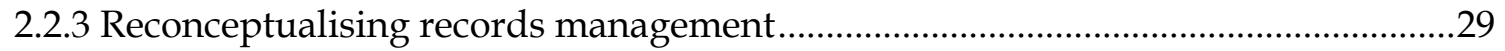

2.2.4 Personal information management strategies ...............................................................

2.2.5 Understanding the recordkeeping behaviours of individuals ....................................36

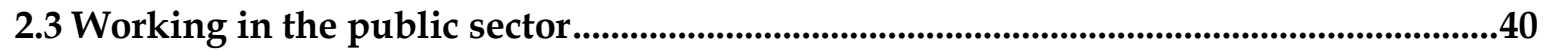


2.3.1 The nature of recordkeeping in the public sector . .40

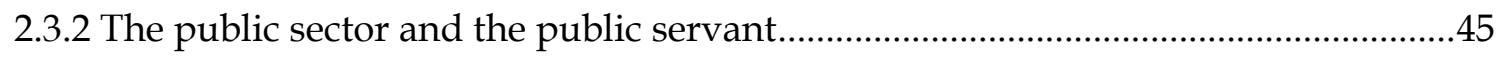

2.3.3 Front-line public servants in ICT-enabled agencies.................................................... 48

2.4 Recordkeeping behaviours in the public sector..........................................................53

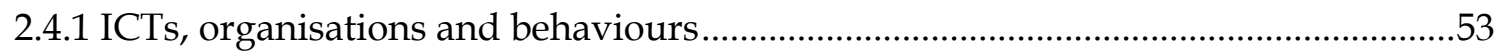

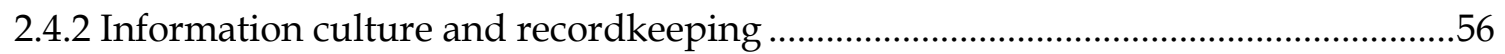

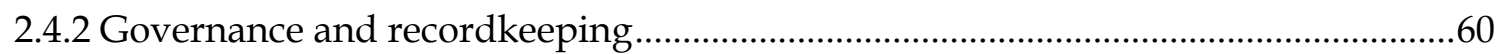

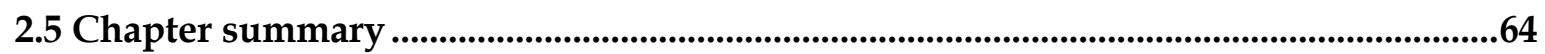

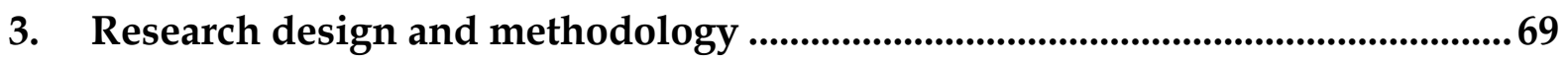

3.1 Introduction to this chapter ........................................................................................................69

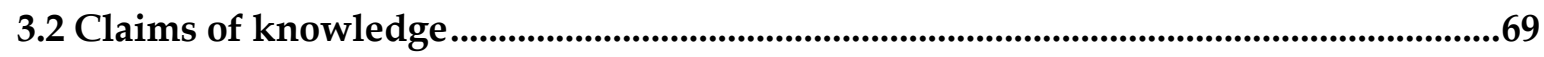

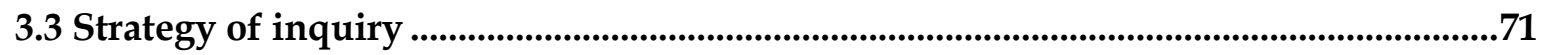

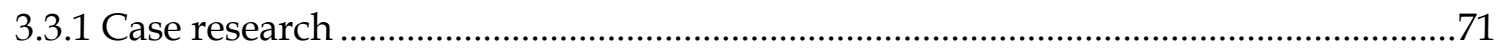

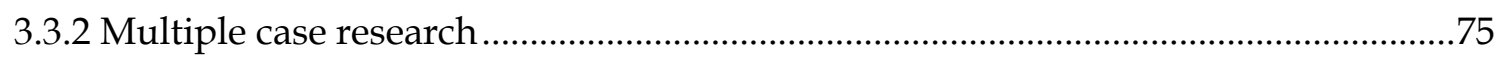

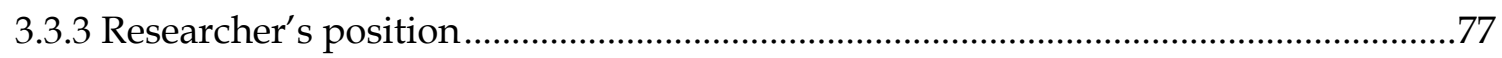

3.4 Methods of data collection and analysis...................................................................................77

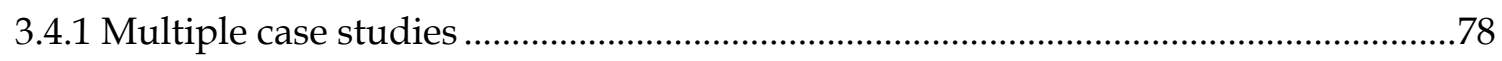

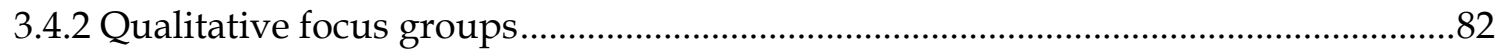

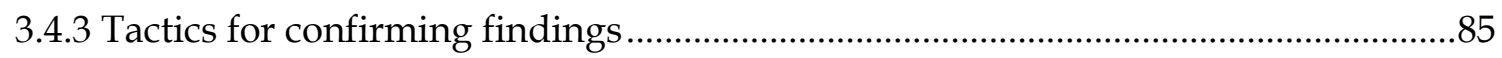

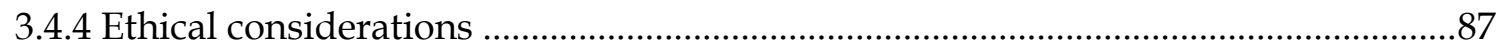

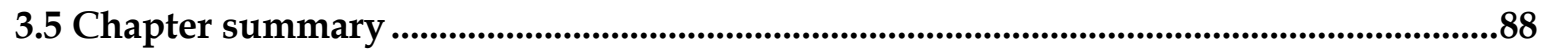

4. Recordkeeping behaviours at Environmental Risk Management Authority.. 90

4.1 The Environmental Risk Management Authority ........................................................90

4.1.1 Information management at ERMA New Zealand...................................................91

4.1.2 Information technology at ERMA New Zealand ....................................................93

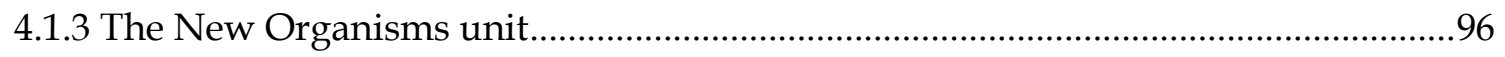

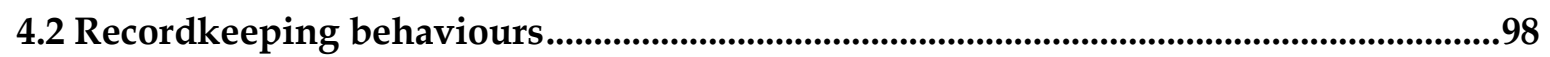

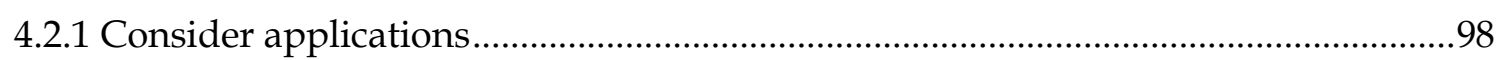

4.2.2. Monitor and review the enforcement of the HSNO Act ........................................109

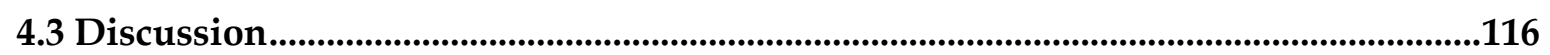

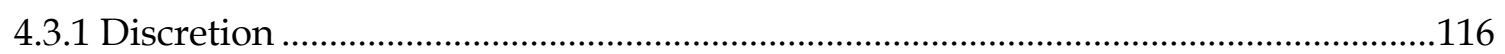

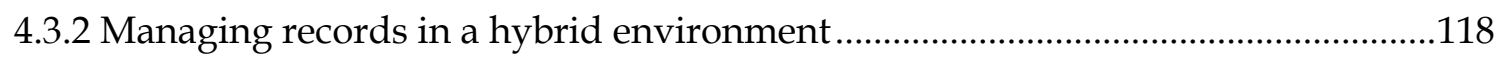

5. Recordkeeping behaviours at Te Puni Kōkiri ................................................. 122

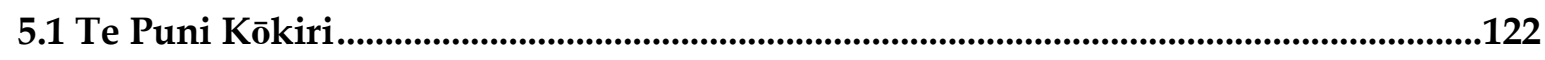

5.1.1 Information management at Te Puni Kōkiri ...........................................................124 
5.1.2 Information technology at Te Puni Kōkiri 125

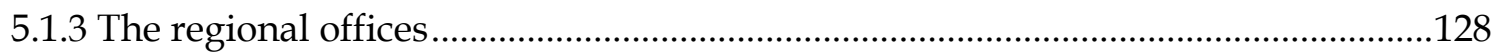

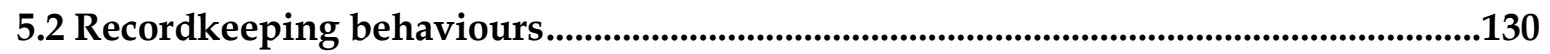

5.2.1 Assist Māori to develop and implement initiatives .................................................131

5.2.2 Gather information to inform policy initiatives and developments .......................135

5.2.3 Liaise between Government and Māori organisations and communities...............139

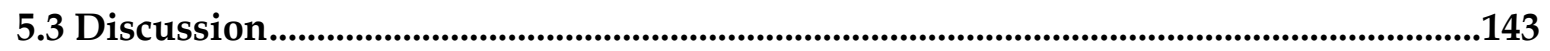

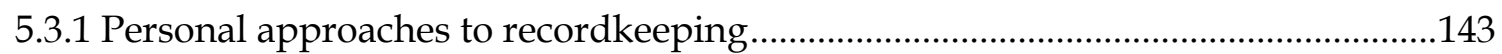

5.3.2 An evolving ICT-enabled work environment .........................................................146

6. Recordkeeping behaviours at Inland Revenue ...........................................149

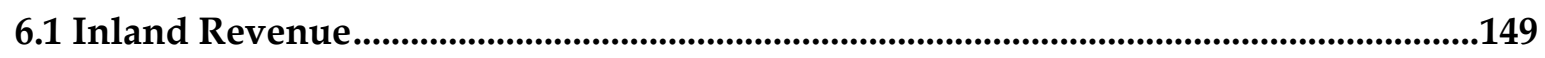

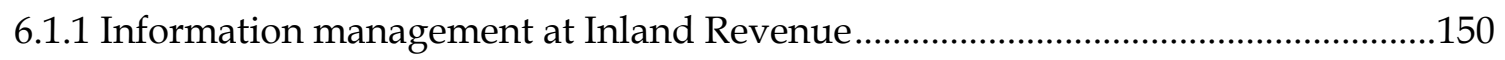

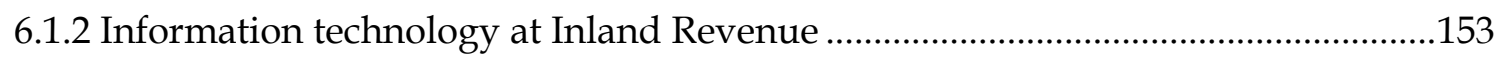

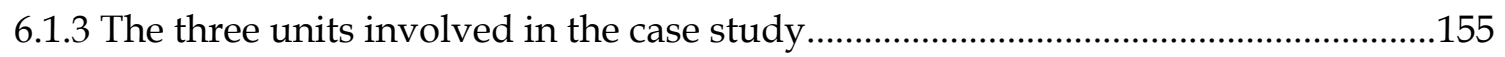

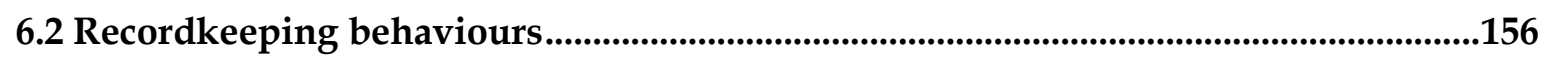

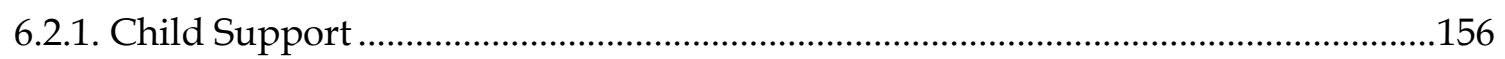

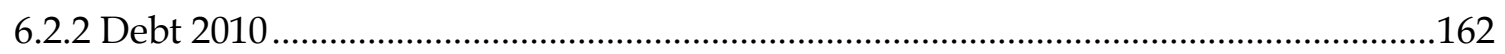

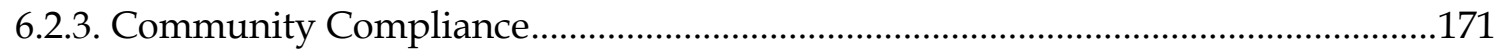

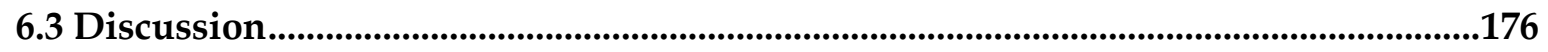

6.3.1. Front-line staff were highly motivated to protect customer details ........................176

6.3.2. Staff made concessions for the limitations of ICTs ....................................................179

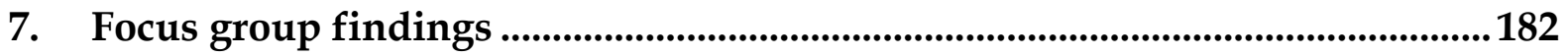

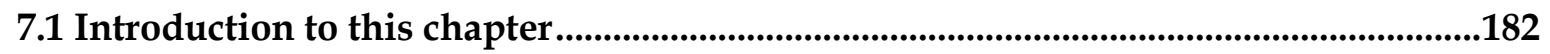

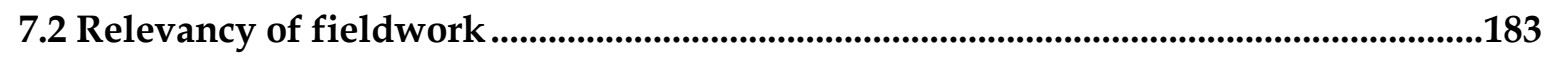

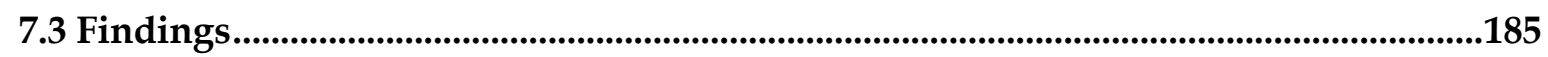

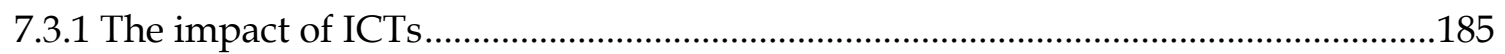

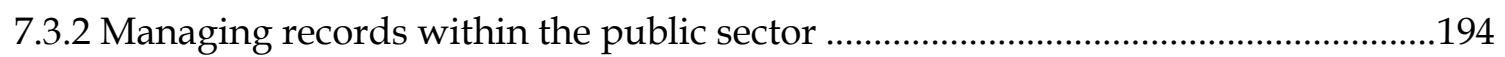

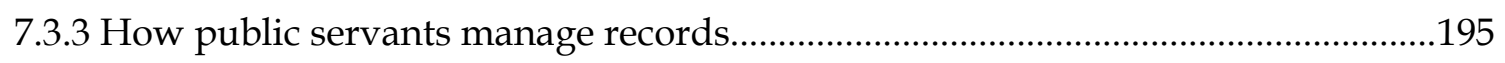

7.3.4 The influence of records managers on staff recordkeeping behaviours ..................197

7.3.5 The role and nature of the governance framework ................................................199

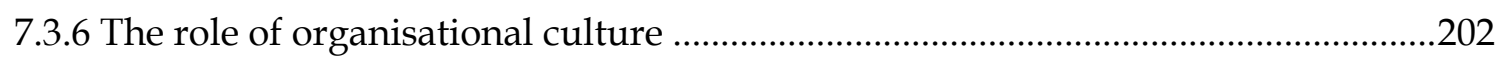

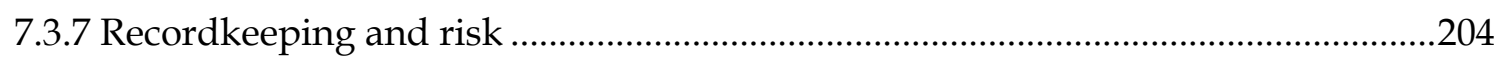

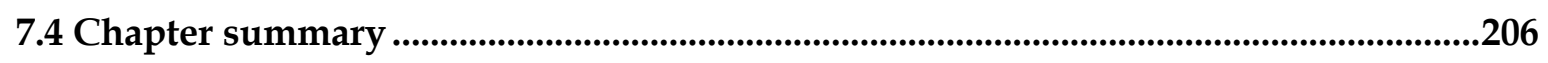

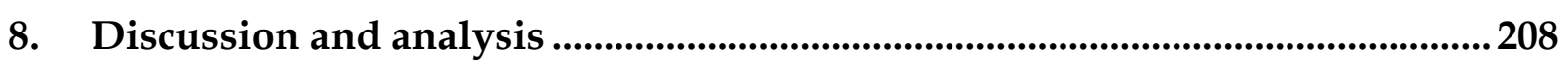

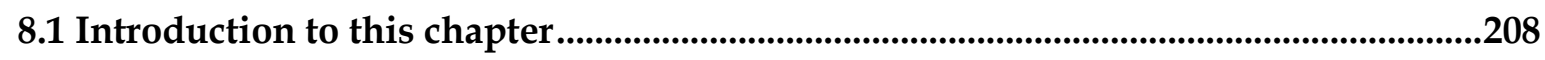


8.2 Recordkeeping informatics .............................................................................................208

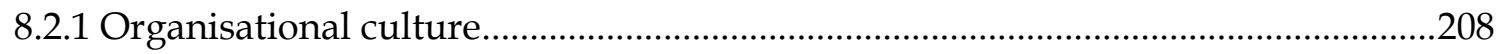

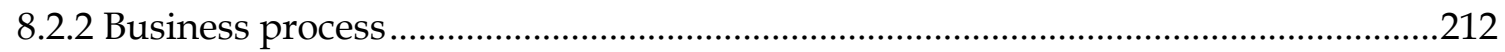

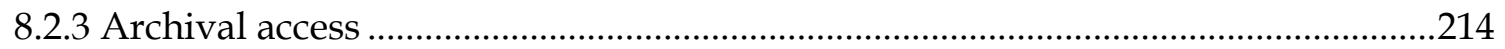

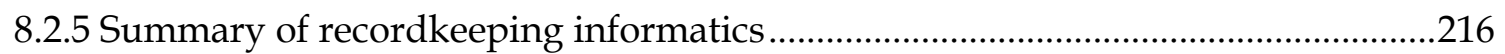

8.3 Personal information management strategies ..........................................................217

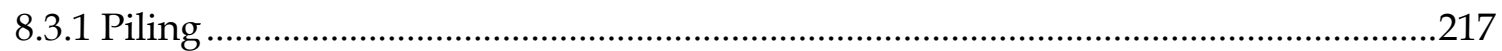

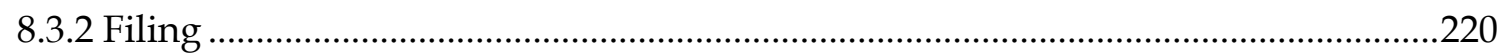

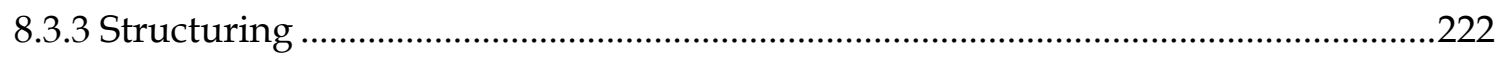

8.3.4 Summary of personal information management strategies ....................................224

8.4 Influencing factors on front-line public servants' decision-making behaviours.....225

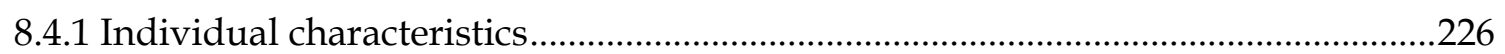

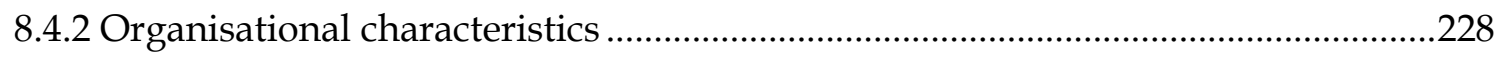

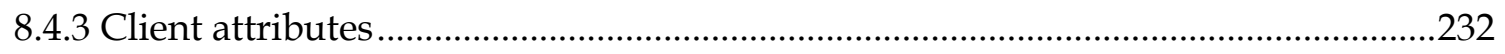

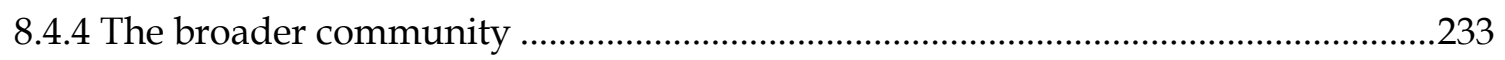

8.4.5 Summary of influencing factors on front-line public servants' decision-making

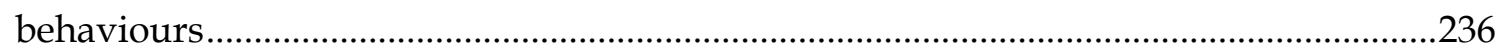

8.5 Recordkeeping and governance .........................................................................................237

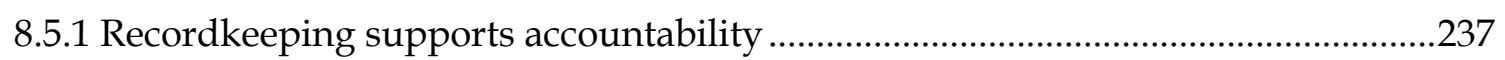

8.5.2 Recordkeeping supports compliance with legislation .............................................239

8.5.3 Recordkeeping provides evidence of business activities ...........................................239

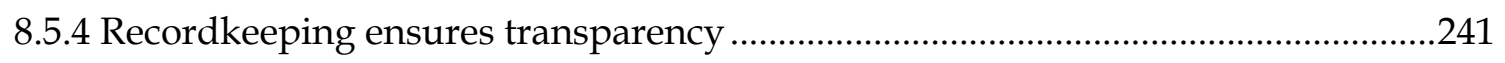

8.5.5 Recordkeeping protects secure and restricted records.............................................242

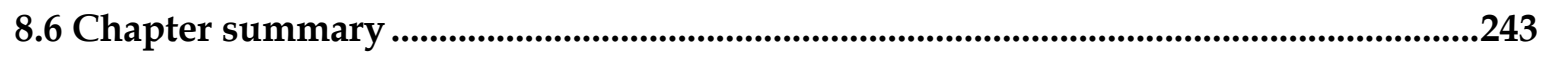

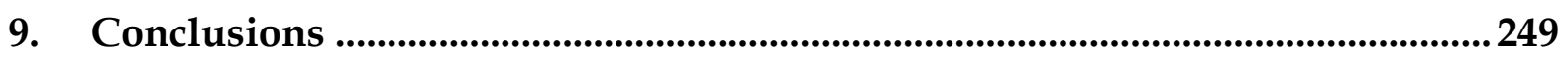

9.1 Introduction to this chapter ....................................................................................................249

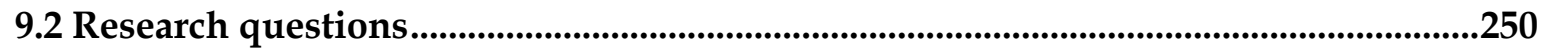

9.2.1 What recordkeeping behaviours do New Zealand front-line public servants

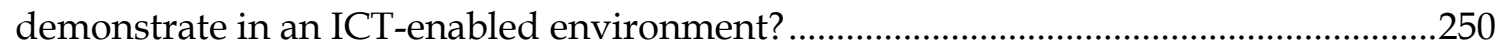

9.2.2 How can these recordkeeping behaviours be understood?.....................................251

9.2.3 What are the governance implications of the recordkeeping behaviours of New

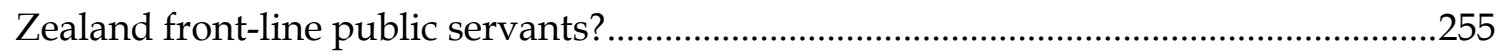

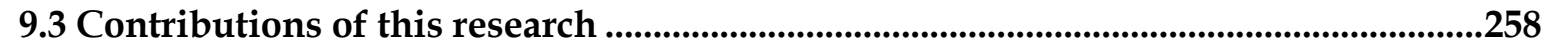

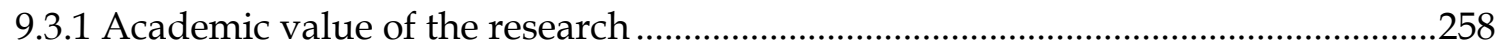

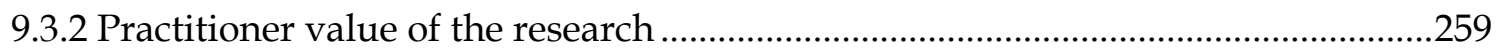




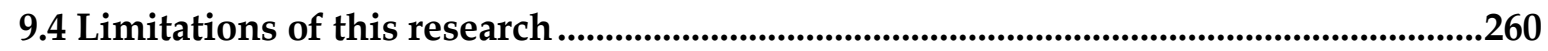

9.5 Future research opportunities ................................................................................................261

Appendix A: Case study participants ............................................................................. 265

Appendix B: Case study interview questions ................................................................. 267

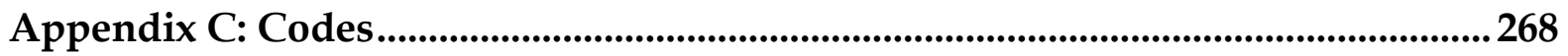

Appendix D: Focus group participants ...........................................................................269

Appendix E: Discussion topics discussed at focus group 1...................................... 272

Appendix F: Organisations contacted for focus group 2........................................... 273

Appendix G: Slide deck for focus groups 2 and 3 ...................................................... 275

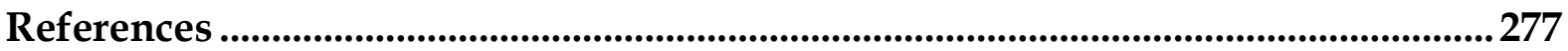




\section{List of Figures}

Figure 2.1: The records continuum (Upward, 2000, 2010) ........................................... 28

Figure 2.2: A conceptual model for personal information management of office support staff workers (Pare, 2011, p. 143) ...................................................................... 34

Figure 2.3: Information culture framework (Oliver \& Foscarini, 2014, p. 18; Oliver et

al., 2018) 59

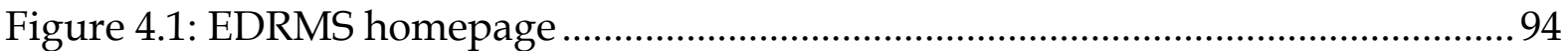

Figure 4.2: New document metadata profile, including default metadata .................. 95

Figure 4.3: Example of email metadata profile in the EDRMS .......................................95

Figure 5.1: The EDRMS homepage (Department of Internal Affairs, 2005)............... 125

Figure 5.2: Te Puni Kōkiri work area, showing large filing cabinets ...........................130

Figure 6.1: Inland Revenue's functional areas (Inland Revenue, 2014c, p. 9)..........150

Figure 8.1: Information security poster at Inland Revenue ........................................ 230

Figure 8.2: Influencing factors on recordkeeping behaviours of New Zealand frontline public servants in ICT-enabled environments......................................................... 246 


\section{List of Tables}

Table 2.1: Selection of salient personal information management strategies 32

Table 2.2: Characteristics of street-, screen- and system-level bureaucracies (Bovens \& Zouridis, 2002, p. 180; Reddick, Abdelsalam, \& Elkadi, 2011, p. 393) ...................... 49

Table 2.3: Key ways that recordkeeping contributes to governance............................ 61

Table 3.1: Case research strategy characteristics (Benbasat et al., 1987, p. 371) ........72

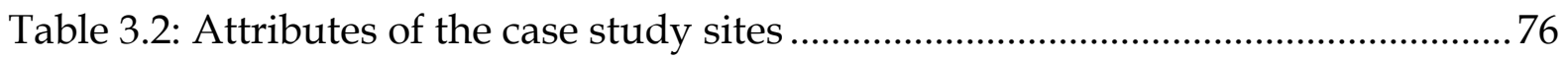

Table 4.1: ERMA New Zealand application process ................................................... 98

Table 5.1: The EDRMS document metadata (Te Puni Kōkiri, n.d.)............................ 127

Table 6.1: Inland Revenue ICTs used by participants .............................................. 154 


\section{List of Vignettes}

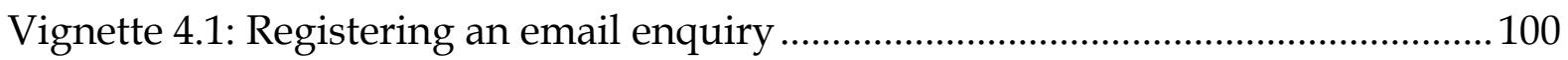

Vignette 4.2: Managing a completed New Organism application ................................103

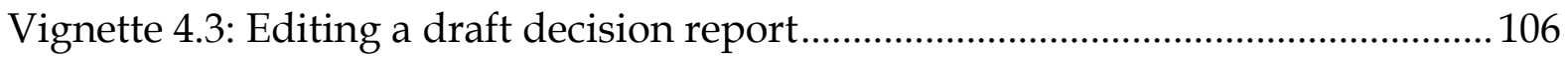

Vignette 4.4: Saving documents to personal drive .........................................................108

Vignette 4.5: Preparing summaries of recent incidents ................................................. 111

Vignette 4.6: Preparing communications with external organisation...........................113

Vignette 4.7: Identifying locations to store digital records..........................................119

Vignette 5.1: Responding to an email query .................................................................. 131

Vignette 5.2: Entering information into the funding management system ................134

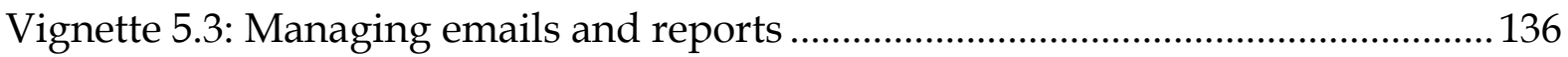

Vignette 5.4: Capturing details from a phone conversation with a member of the

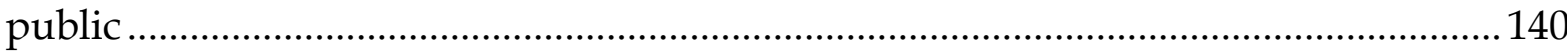

Vignette 5.5: Capturing details from a member of the public's visit ..........................141

Vignette 6.1: Responding to a query from a custodial parent......................................158

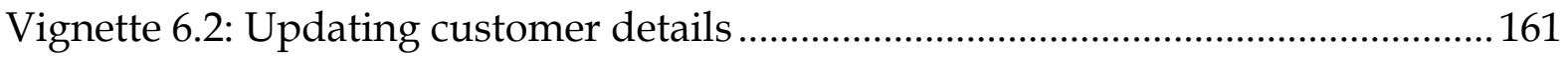

Vignette 6.3: Requesting information from organisations............................................. 164

Vignette 6.4: Reviewing a customer's situation .............................................................. 166

Vignette 6.5: Arranging debt repayments................................................................ 169

Vignette 6.6: Preparing information for company visits................................................172

Vignette 6.7: Preparing a submission report ……………………………………...... 175

Vignette 8.1: Organisational poster for 'Keeping Our Information Safe' ................... 229

Vignette 8.2: Information sharing between agencies................................................... 234 


\section{Introduction}

\subsection{Introduction to this chapter}

What do we know about how front-line public servants manage records? How can we understand these behaviours within Information and Communication Technology (ICT) enabled agencies and what are the governance implications of these behaviours? Understanding the recordkeeping behaviours of front-line public servants and the broader governance implications of them provides an empirical understanding of the administrative functioning of public agencies within the digital age, while providing further knowledge about the role that individuals play in creating, managing and using documented evidence of government activities. This study attempts to respond to these questions through a series of case studies and focus groups.

\subsection{Motivations for the study}

Public agencies rely on evidence of activities, in the form of public records, to coordinate activities, track resources, establish precedents, monitor performance as well as maintain consistency and continuity. In many public agencies, the responsibilities to create and manage public records are delegated to front-line public servants. It is these front-line public servants who decide what details to retain or discard, either for immediate use or as records of long-term value, on a daily basis (White, 2007, p. 258). However, to date, we know little about the working realities of the recordkeeping behaviours of these individuals.

Understanding how front-line public servants create, manage and use records provides unique insights into the mechanics of the public sector and of public agencies in the digital age. ICTs and the changes to work practices that they introduce have significantly altered the concept of a record and many recordkeeping 
processes, in addition to changing the working environments within public agencies and the relationships between front-line public servants and citizens. Prior to the use of ICTs and for much of the previous century, public servants had secretaries, record clerks and records managers to create and manage physical records on their behalf (Cook, 1994, p. 302). As organisations progressively introduced ICTs for undertaking business activities, the nature of records changed, agencies created, managed and used records in digital formats, and staff members became increasingly involved in the day-to-day creation and management of public records.

While technical innovations and ICT-enabled organisations have been occurring for decades, and as traditional approaches for managing records become less effective, there continues to be a need to explore new ways and models of working that emphasise the importance of information activities from different disciplinary perspectives (Moss, Endicott-Popovsky, \& Dupuis, 2018, p. XVI) Over twenty years ago, Cook (1994, p. 301) described the situation: "For the first time in 3500 years of records management and archival activity, we have too much rather than too little information. For the first time, we have records that do not exist to the human eye".

Research conducted over the past ten to fifteen years provides insights into the recordkeeping behaviours of individuals; including research that explores EDRMS use by individuals (Joseph, 2010; Lewellen, 2015), the impact of information culture on records management (Oliver, 2005, 2008, 2011; Oliver \& Foscarini, 2014; Sundqvist \& Svärd, 2016; Svärd, 2014; T. Wright, 2013), as well as exploration of genre theory and information studies (Andersen, 2015; Foscarini, 2013; Spinuzzi, 2003). Sundqvist and Svärd (2016, p. 9) argued that despite investments in technology and legal frameworks that controlled the management of information resources, and the significant knowledge produced within the records management community, attaining good information and records management within 
organisations remained a challenge, and they suggested that other, less tangible, factors impacted on the information and recordkeeping behaviours of individuals.

While existing knowledge in the recordkeeping environment is valuable in providing contextual information surrounding recordkeeping behaviours of individuals, there is little empirical research available that helps us to understand and explain how the practical aspects of front-line public servants create, manage and use records, across both digital and paper-based formats and using the multitude of ICTs available at their desktops. Furthermore, research is lacking that explores the effects of these recordkeeping actions within the context of the New Zealand ICT-enabled public agencies and the broader NZ public sector as a whole. As Strong et al. (2014, p. 55) highlighted it is less common to examine the micro-level (individual user) recordkeeping behaviours alongside the macro-level institutions in which they are embedded.

However, there are multiple calls for research in three closely related areas: on the behaviours of front-line public servants, on the impacts that the use of ICTs introduce for front-line public servants and recordkeeping, and on the relationships between of e-government, ICTs and recordkeeping.

Ethnographic studies on the behaviours of front-line public servants, with particular focus on the use of ICTs suggested that further research was required to provide insights and understanding into the effects of governance strategies on individual practices (Lundberg \& Syltevik, 2016, p. 163; Pors, 2015, p. 188). In addition, French and Williamson (2016, p. 737) claimed that the information practices of welfare workers in the Australian services sector has been limited so far, reflecting a similar situation in New Zealand. 
Furthermore, Buffat (2015, p. 9) suggested that as existing knowledge is inconclusive regarding the impacts of ICTs on front-line discretion, more empirical research is needed; in particular, research that addresses issues of discretion and accountability in ICT-enabled public agencies.

Kallberg (2013, p. 91) identified the need for further research into the relationship between e-government and recordkeeping. She suggested that the use of ICTs to deliver services to citizens and businesses raises practical and theoretical recordkeeping issues regarding the context of the creation of records and recordkeeping legislation.

This multidisciplinary research draws on the public management, information management and information systems disciplines to respond to these calls for empirical research.

\subsection{Terminology and key concepts}

This section defines several key concepts that are discussed throughout this study and to set the context for this research.

\subsubsection{New Zealand public sector}

The public sector encompasses the institutions, government departments, museums, schools, hospitals, and other specialist bodies that regulate everything from casinos to civil aviation (R. Shaw \& Eichbaum, 2008, pp. 5-6). The focus of this study is the New Zealand state sector, which includes the core public sector departments as well as Crown entities, State-owned enterprises, non-public service departments, the offices of Parliament and the Reserve Bank of New Zealand. 
As a consequence of the use of ICTs within the state sector, Noordegraaf (2016, pp. 790-791) suggested that professional work in public agencies has been fundamentally reconfigured in three ways: professional work might have been reorganised, re-stratified, or relocated. In particular, Noordegraaf (2016, p. 799) argued the introduction of ICTs contributed to the digital relocation of public professional work; for example, universities offer digital study programs that can be followed at a distance and ICTs affect how medical diagnosis and treatment take place (Noordegraaf, 2016, p. 796). Katsonis and Sullivan (2014, p. 16) also explored changes to the public service of the 21st century and identified factors impacting this change, including the impact of globalisation, the ageing population, changing citizen expectations for more personalised services and technological change. A significant component of these changes proposed by Katsonis and Sullivan (2014, p. 16) related to the use of ICTs to administer and deliver public services, including the uptake of smart devices, the popularity of social media, the emergence of cloud computing, and the rise of big data and analytics, giving rise to new skill sets that sit alongside the traditional ones expected of public servants.

The increasing use of digital tools and applications in public administration, the shift in how government services are delivered, and the provision of governmental online services is referred to as 'e-government' (Buffat, 2015, p. 150). In New Zealand, egovernment has been defined as encompassing a broad range of government activities within the New Zealand public sector that involve the use of ICTs as a means to improve operational performance (O'Neill, 2009, p. 15).

\subsubsection{Information and Communication Technologies}

Information and Communication Technologies (ICTs) is defined for the purposes of this study as the range of technologies that ensure information is available across a 
variety of devices and that enable communication between people and between systems (O’Neill, 2009, p. 16).

Information, in varying forms, is a key component of any ICT or e-government initiative. Within New Zealand, the Government ICT Strategy was revised in 2015 to support the government's aim of an ICT-enabled transformation of public services to New Zealanders; one of the four high-level opportunities of this strategy was unlocking the value of information (New Zealand Government Chief Information Officer, 2016a), reinforcing the importance of information including physical and digital records, within the digital age and within the New Zealand public sector.

The use of ICTs and e-government initiatives has changed how the public sector functions, particularly for those public servants representing the government in the delivery of services, and for citizens engaging with government.

\subsubsection{Front-line public servants}

This study is specifically focused on those employees within the New Zealand state sector who interact with members of the public on a regular basis as a significant component of their role. In 2015, the New Zealand state sector employed 302,000 people, approximately 13 percent of the total employed workforce, across 255 different occupations (State Services Commission, 2015, p. 8).

As Carroll and Siegel (1999) highlighted most attention in both the popular media and academic work is on high-profile politicians and senior public servants. Whilst understandable as it is this group of public servants who make significant decisions with far-reaching implications, this ignores the large number of people who interpret the policies, deliver public services and who represent the face of government to citizens receiving these public services. 
All of the participants in the case studies held roles that included responsibilities to interact with members of the public on a regular basis; participants also defined themselves as front-line public servants. While the majority of vignettes within the three case study chapters of this thesis describe interactions between the participants and members of the public, some of the vignettes illustrate tasks conducted by the participants that do not include interactions with members of the public.

\subsubsection{Behaviours}

What individuals do and the ways they act within organisations are important in order to build an understanding of the way organisations function, and equally important when attempting to understand factors that influence those behaviours.

In an exploration of the individual in the organisation, Furnham $(2005$, p. 52) defined organisational or work psychology as being the study of how organisations influence the thoughts (including the beliefs and values), feelings and, importantly, the behaviours of all those working in the organisation. While a person can have thousands of attitudes, organisational behaviour focuses attention on a limited number of work-related behaviours within the workplace. Robbins and Judge (2008, p. 2) explained that organisational behaviour is specifically concerned with employment-related situations, and consequently emphasises behaviour in relation to jobs, work, absenteeism, employment turnover, productivity, human performance and management.

When focusing on the behaviours of individuals within public agencies the German sociologist, Max Weber, provides a valuable starting point. Weber viewed bureaucracy as a mechanistic construct, where an optimal degree of managerial control was exercised over large numbers of 'functionaries' whose efforts were directed towards the achievement of the 'owner's' purpose (Gregory, 1982, p. 3). In 
this depersonalised system of authority, the bureaucrat behaved not as an individual social being whose attitudes, values, expectations and preferences were shaped by his or her life experiences, but as a functionary competently fulfilling the legal requirements of the office (Gregory, 1982, p. 4).

Herring (1936, p. 7) described the concept of administrative discretion, which he characterised as a process whereby Congress passed a statute setting forth a general principle, the details were filled in by supplementary regulation, while the public servant was left to decide as to the conditions that necessitate the application of the law. Herring (1936, pp. 7-8) suggested that public servants are in a better position than the legislators to perform these duties as their daily occupation brings them into direct contact with the situation that the law is intended to meet. Thus, Herring described a shift from the Weberian approach where public servants were seen primarily as functionaries, fulfilling legal requirements, to one where bureaucrats were acknowledged as social beings who behaved in ways that brought their life experiences to their roles and had opportunity to influence policy decisions.

In the 1950s organisational theorist, Simon, began to systematically study organisations, with particular focus on decision-making, and identified the specific and important role that front-line public servants held. Simon $(1976$, p. 2) found that the physical task of carrying out an organisation's objectives often fell on those individuals at the lowest levels of the administrative hierarchy; in this way, all employees within an organisation played an essential role in the accomplishment of organisational objectives.

Lipsky (1980, p. 3) acknowledged this important role of front-line public servants, describing front-line public servants as 'street-level bureaucrats'. Lipsky (1980, p. xii) argued that the decisions of street-level bureaucrats, the routines they established 
and the devices they used to cope with uncertainties and work pressures effectively become the public policies that they carried out:

“...Public policy is not best understood as made in legislatures or top-floor suites of high-ranking administrators, because in important ways it is actually made in the crowded offices and daily encounters of street level workers".

Drawing on previous research exploring the nature of public servants behaviours within organisations, the unit of analysis for this study is the individual within the organisation, in particular NZ state sector agencies, and more specifically, front-line public servants. Furthermore, this study focuses on one particular set of behaviours; the recordkeeping behaviours undertaken by these front-line public servants.

\subsubsection{Recordkeeping}

It is important to be explicit about what recordkeeping means within the context of this study.

Based on the discussion by Upward, Reed, Oliver and Evans (2013, p. 38), recordkeeping is defined for the purposes of this study as "the processes by which we create, capture, organise and disseminate recorded information as evidence". It is the creation, management and ongoing use of recorded information by individuals that is central to this study; processes that bridge recordkeeping, archives management, information systems and business processes within the New Zealand public sector (Upward et al., 2013, p. 38).

This study uses the phrase recordkeeping system to refer to the broad framework used within organisations to create, manage and provide access to use records over time. Within this context, recordkeeping systems include: 
- recordkeeping professionals as well as records creators and users,

- a set of authorised policies, assigned responsibilities and delegations of authority,

- procedures, practices manuals and user guidelines that are used to promulgate the policies,

- the records (in both physical and digital formats),

- specialised information and records systems used to control the physical and digital records, which may or may not include an Electronic Document and Records Management System (EDRMS), and

- equipment, including software, hardware and stationary, and other resources (International Council on Archives, 2008, p. 64).

Recordkeeping is consciously spelled as one word throughout this study reflecting the interrelated processes for the creation, management and use of records, cognisant of spelling set out by the records continuum theory. The alternative spelling, using two words, record keeping, refers to the keeping of records as physical objects (Upward et al., 2013; Wareham, 2002).

Recordkeeping behaviours tend to be embedded in business processes; the saving of files, naming documents, filing, completing metadata and other behaviours are often considered routine and mundane (Trace, 2002, p. 159). However, it is these behaviours that have a direct correlation to the functioning of agencies as these agencies rely on public records as evidence of activities. 


\subsubsection{Governance}

This study is also focused on exploring the governance implications of the recordkeeping behaviours of New Zealand front-line public servants. Shaw and Eichbaum (R. Shaw \& Eichbaum, 2008, p. 278) used the term governance to describe the wider constellation of policy actors, the formal and informal institutional arrangements within which they interrelate, and the type and quality of those interrelationships. O'Neill (2009, p. 14) defined governance as the framework that public servants operated in including, but not restricted to, accountability and responsibility regimes, constitutional protocols, policy and operational functions, systems and processes. Within the context of this study, the term governance draws on these definitions and is used to reflect not just the activities and organisation of government agencies, but also to take into consideration the broader arrangements and relationships between the public bodies that shape the scope and nature of public administrative duties (O'Neill, 2009, p. 18; R. Shaw \& Eichbaum, 2008, p. 278). Exploring the implications from this perspective provided an opportunity to explore principle governance mechanisms linked to recordkeeping, in particular law and ethics.

\subsection{The New Zealand public sector context for recordkeeping}

The legislative framework for recordkeeping makes recordkeeping in the New Zealand public sector unique and further defines the context for this study. In 2011, the New Zealand government approved a set of principles for managing the data and information that it holds. These principles established that government data and information should be open, readily available, well managed, reasonably priced and re-usable unless there are necessary reasons for its protection; personal and classified information should remain protected, and government data and information should be trusted and authoritative (New Zealand Government Chief Information Officer, 
2016b). These principles for managing data and information held by the New Zealand Government also define the legislative framework for data and information managed by the New Zealand state sector, based on the Copyright Act 1994, the Official Information Act 1982, the Privacy Act 1993, the Public Records Act 2005 and the Statistics Act 1975. This framework, with the inclusion of the Contract and Commercial Law Act 2017, forms the basis of the legislative framework underpinning this study.

\subsubsection{Copyright Act 1994}

The Copyright Act 1994 consolidated and amended previous copyright laws (Copyright Act, 1994). Copyright is a property right that exists in original works of literary, dramatic, musical, or artistic works, sound recordings, films, communication works, and typographical arrangements of published editions (Copyright Act, 1994).

\subsubsection{Official Information Act 1982}

Access to information held by New Zealand government agencies is governed by the Official Information Act 1982 (OIA), which provides members of the public with the right to access government information held about themselves and about the activities of government agencies (Official Information Act, 1982). The Act is premised on the belief that access to official information is essential to holding governments to account and is critical to people's ability to participate in the policy process (R. Shaw \& Eichbaum, 2008). The Act requires that all official information is made available upon request unless there is good reason for withholding it and that agencies are responsible for releasing this information when required.

\subsubsection{Privacy Act 1993}


The Privacy Act 1993 is primarily a data protection statute, concerned with the protection of personal information about individuals, and defines personal information as the information needed to identify that person or be capable of identifying that person (Privacy Act, 1993). The Act contains twelve privacy principles dealing with collecting, holding, using, disclosing of personal information and assigning unique identifiers, such as Inland Revenue numbers and passport numbers.

\subsubsection{Public Records Act 2005}

The Public Records Act 2005 (PRA) outlines the recordkeeping requirements for all public offices within New Zealand, including local government and schools (Public Records Act, 2005). The PRA replaced the Archives Act of 1957 in 2005, reflecting developments in technology and recordkeeping, including the records continuum theory, while continuing the general position that the Chief Archivist must authorise the disposal of records. The PRA outlined responsibilities for all public offices to create and maintain full and accurate records of their activities in accordance with normal, prudent business practice, and that public records remain accessible over time.

There is evidence that just as the OIA and the Privacy Act are vehicles for the public to question how the government is handling public information, the PRA has a similar role, and that this is increasingly being employed by members of the public. Archives New Zealand (2015, p. 10) stated that in 2014/2105 requests for intervention from the Chief Archivist by individuals about the recordkeeping behaviours within public offices and local authorities increased from two requests in 2013/2104 to 19 requests. Archives New Zealand (2015, p. 10) suggested that the increase in these requests for intervention reflected growing public attention on the 
importance of good information management by government and may be a result of a public desire to have public records accessible and safe for future generations.

\subsubsection{Statistics Act 1975}

The Statistics Act 1975 amended the Statistics Act 1955, made provisions for official statistics, for the Statistics New Zealand department as well as the role and independence of the office of the Government Statistician (Statistics Act, 1975). Official statistics are defined within the Act as statistics derived by government departments from statistical surveys, administrative and registration records, as well as other forms and papers the statistical analyses of which are regularly published (Statistics Act, 1975).

\subsubsection{Contract and Commercial Law Act}

The Electronic Transactions Act 2002 was repealed in 2017, by section 345(1)(e) of the Contract and Commercial Law Act 2017. In relation to electronic transactions, the Act facilitates the use of ICTs by reducing uncertainty regarding the legal effect of information that is in electronic form or that is communicated by electronic means; clarify the time and place of dispatch and receipt of electronic communications; and provides that certain paper-based legal requirements may be met by using electronic technology as long as it is functionally equivalent (Contract and Commercial Law Act, 2017).

\subsubsection{Relationship between these pieces of legislation}

In theory, these pieces of legislation have a mutually supportive relationship. The PRA informs what information is retained within agencies, thus forming the foundation for the OIA, which governs how decisions are made on what is released publicly. The Privacy Act protects information about individuals and places 
restrictions on what can and cannot be released into the public arena when public records contain personal information. The Statistics Act makes provisions specifically for creation, management and use of data derived by government departments from statistical surveys. Use and re-use of government works, including documents and data, is defined by the Copyright Act. The Contract and Commercial Law Act sets out that information is not denied legal effect solely because it is in digital form. In reality, however, there are suggestions that these pieces of legislation may place conflicting requirements on agencies and may not reflect current ICT-enabled work practices.

In research into the OIA in New Zealand, White (2007, p. 257) suggested that the OIA and the PRA were set too far from the working reality of the public sector. White (2007, p. 258) argued that the idea that all records were kept and that destruction only occurred with authority from the Chief Archivist was out of step with the way that public servants created and deleted records, particularly in ICTenabled public agencies.

Furthermore, the Law Commission (2012, p. 27) suggested that there was anecdotal evidence that at times individuals subject to official information legislation were tempted not to create or capture information, for example individuals provided advice, but tended to convey this orally to avoid disclosure, meaning records were not created. White (2007, p. 271) also suggested that at times the OIA impacted how and what information was created and captured, even though this contravened the spirit of both the OIA and the PRA.

\subsection{The research question}

The purpose of this study is to explore the recordkeeping behaviours of New Zealand front-line public servants within ICT-enabled state sector agencies in order 
to better understand these behaviours and their associated governance implications. Underpinning this research purpose, the primary research question for this study is:

- What recordkeeping behaviours do New Zealand front-line public servants demonstrate in an ICT-enabled environment?

Converted into research objectives, the aims of the research are:

- To develop an empirical understanding of recordkeeping behaviours of New Zealand front-line public servants within ICT-enabled environments.

- To explore the governance implications of the recordkeeping behaviours of New Zealand front-line public servants.

\subsection{The research approach}

The approach for this inductive study consists of two key data collection phases. The study began with three case studies, involving in-depth interviews and nonparticipant observations that explored the behaviours of front-line public servants creating, managing and using records. Existing theories in the fields of public administration and recordkeeping were used to explore the behaviours identified during this fieldwork. This study employed a case study approach as this research strategy supports empirical inquiries investigating contemporary phenomenon, in this case recordkeeping behaviours, in depth and within their real-world context (Yin, 2014, p. 16).

The second data collection phase involved a series of focus groups that explored the governance implications of the individual behaviours that were observed during the fieldwork, providing a macro-level perspective on the findings of the case studies. This phase of the study utilised focus groups as they provided an opportunity to 
collect multiple understandings and meanings within a group environment and to explore the degree of consensus on the discussion topics.

The nature of this study is extensive and multidisciplinary. Therefore, it was necessary at the outset of the research to establish some boundaries so that the research problem was able to be addressed in a manageable and meaningful way.

The focus of this study is on recordkeeping behaviours. As outlined above, behaviours are understood for the context of this research as what individuals do within employment-related situations. The specific behaviours that form the central focus of this study relate to the processes by which recorded information is created, managed and used as evidence.

Limiting the study to the New Zealand context was a deliberate choice. As an information management professional, the researcher brings existing knowledge and familiarity with the relevant legislation, the New Zealand state sector as well as the recordkeeping environment within New Zealand. Focusing the research in New Zealand provided an opportunity to delve deeper into the specific state sector and recordkeeping contexts at play that would have been difficult within an international study.

This study purposefully concentrated on front-line public servants. Front-line public servants provide an interface between public service internal procedures and information, and the users of services they encounter on a daily basis (Bjerregaard \& Klitmoller, 2010, p. 421), or as Snellen (1998, p. 500) described, the street-level bureaucrat is the link between two information streams: the internal process of information about rules, formal categories and procedures and the external process of information about the circumstances and wishes of clients. Investigating the realities of work for those public servants who are directly engaged with citizens, 
while also moving beyond examining records going into and out of an EDRMS to consider the broad recordkeeping systems within organisations and across the public sector provides a unique perspective to develop an understanding of frontline public servants' recordkeeping behaviours.

The selection of research sites was limited to three case studies, focused on teams of public servants who worked directly with members of the public within the New Zealand state services. The three organisations involved included two public service departments (Inland Revenue Department and Te Puni Kōkiri, the Ministry of Māori Development) and one statutory crown entity (Environmental Risk Management Authority). The focus on state sector agencies was intentional as public service departments and crown entities make up a significant component of the New Zealand central government and deal substantially with the public through public service provision. In addition, these agencies operated within a similar legislative environment.

This study specifically focused on exploring the governance implications of New Zealand front-line public servants' recordkeeping behaviours. As Meijer and Homburg (2008, p. 708) highlighted many of the fundamental changes that coincide with e-government technologies are transforming public agencies for democracy, bureaucracy and other cornerstones of public administration, including governance. However, a difficulty in understanding e-government is that one has to understand the technological changes that are taking place at a micro level in public agencies to comprehend the institutional transformations in public administration at a macro level (Meijer \& Homburg, 2008, p. 708). Understanding recordkeeping behaviours from this macro-perspective, in addition to the micro-perspective of individuals, provided a unique opportunity to explore the multiple ways that recordkeeping contributes to public governance in the digital age. 


\subsection{The significance of the study}

The outcomes of this study offer both academic and practical benefits. From an academic perspective, this study analyses the empirical data through the application of three disparate theoretical concepts: recordkeeping informatics, personal information management strategies, and Loyens and Maesschalck's (2010, p. 72) set of factors that influence front-line public servants' decision-making behaviours. As a consequence of this analysis, factors influencing the recordkeeping behaviours of front-line public servants are identified and explored. In addition, the application of ethnographically-informed research instruments in public sector recordkeeping provide this study with important insights into recordkeeping behaviours that might not have been identified using alternative research methods. As Cooper (2016, p. 269) highlighted there is a growing movement towards conducting and real benefits as a consequence of employing ethnographic research, or using ethnographicallyinformed research methods, within information organisational settings, including in library, archival and recordkeeping research.

From a practical perspective, this study provides in-depth case studies that explore the recordkeeping behaviours of individuals within the context of ICT-enabled public agencies, providing empirical understandings into the use of ICTs by frontline public servants, the role of recordkeeping functionality within ICTs, as well as an empirical understanding of the changing behaviours of front-line public servants and the changing nature of public records. These learnings provide useful insights for organisations setting information-related standards within New Zealand, for recordkeeping staff setting and regulating policies and procedures within public agencies as well as for software engineers developing ICT-enabled business and recordkeeping systems. 
Understanding the recordkeeping behaviours of individuals from a governance perspective provides an empirical understanding of the role that both legislation and ethics play in the recordkeeping behaviours of front-line public servants. This study provides empirical evidence of the multiple ways that recordkeeping contributes to governance, including how recordkeeping supports accountability, underpins compliance with legislation, provides evidence of business activities, ensures transparency, as well as protects secure and restricted records.

\subsection{Structure of the thesis}

This chapter introduces this study and provides a starting point for the remainder of the thesis. Chapter 2 presents a review of the literature, drawing on relevant research from two key disciplines: public administration and records management. The literature review describes theoretical concepts from both disciplines, providing the analytical framework for this study. Chapter 3 describes the research design and in the process, defines the claims of knowledge that are made, the strategies of inquiry informing the research design and the methods of data collection and analysis used. Chapters 4, 5 and 6 present the three case studies, in the order in which they were conducted. Chapter 7 presents the findings from the focus groups. Chapter 8 analyses the findings from the three case studies and focus groups using the analytical framework as the foundation. The final chapter, chapter 9, outlines the conclusions of this study in relation to the research question and research aims, and discusses the implications for theory, policy and practice. It also summarises the limitations of this study and suggests areas for further research.

\subsection{Chapter summary}

The use of ICTs within government has shifted the creation and management of records from centralised control to individuals; organisations delegate the 
responsibilities to create, manage and use these public records to front-line staff, including deciding what details to retain or discard, either for immediate use or as records of long-term value (Meijer, 2001, p. 263; White, 2007, p. 258). Furthermore, recordkeeping and governance are intrinsically linked; records are a source of legal evidence, and records document the relationships between citizens and the state (Iacovino, 2005, pp. 257-258). However, to date, little is known about the recordkeeping behaviours of front-line public servants or the governance implications of these behaviours. The aim of this research is to address this knowledge gap through a series of case studies and focus groups to develop an understanding of the role that individuals play in creating, managing and using records of government activities and to explore the governance implications of these behaviours. 


\section{Literature Review}

\subsection{Introduction to this chapter}

The literature review is an analytical synthesis of research and theory related to a specific subject of inquiry (Ridley, 2008, pp. 2-4). This literature review draws on relevant research from two key disciplines: public administration and records management; and describes relevant theoretical concepts from both disciplines, providing the context for this study. Each section of this literature review builds on concepts introduced in the previous section, with the aim of presenting a comprehensive understanding of the current knowledge of the recordkeeping behaviours of individuals in the New Zealand public sector.

The first section examines existing research in recordkeeping, providing an overview of the records continuum theory, recordkeeping informatics and personal information management strategies. The second section explores concepts that address the nature of public sector work, paying particular attention to the unique working context for front-line public servants. The third section links the two earlier sections, paying particular attention to related concepts that run through both the public administration and recordkeeping disciplines, including the nature of behaviour with organisations, how front-line public servants' work is changing with increased use of ICTs, information culture as a framework for studying recordkeeping behaviours of front-line public servants, as well as the interdependent association between governance and recordkeeping.

\subsection{Creating, capturing, organising and disseminating recorded information as evidence}

\subsubsection{Records and recordkeeping in the digital age}


Reed (2007, p. 102) argued that a critical characteristic of a record is that it is linked to doing something; that is, it is transactional. Reed (2007, p. 102) explained that it is the transactional nature of records that distinguish them from other information resources, such as data and documents; however, records consist of data, documents and information. In order to further distinguish records from other information resources, Lewellen (2015, p. 10) proposed that data, documents and information are not records until they have been placed under systematic control that can guarantee the key characteristics required for use as evidence.

These key characteristics, regardless of the records' formats, are defined within ISO 15489 (ISO, 2001, p. 7) as records that are authentic, reliable, useable and where their integrity is protected for as long as required. The recordkeeping system supporting the records must align with the complementary principles of reliability, integrity, compliance, comprehensiveness and being systematic (ISO, 2001, pp. 8-9). These core characteristics of records and recordkeeping system principles apply equally to physical and to digital contexts; however, the rapid technological changes since the 1980s and the increasing dependence of society on ICTs present a number of potential challenges to managing digital records.

As described by Evans et al. (2014, p. 206), continuous ICT innovations and an ongoing drive for increasingly digital recordkeeping have diminished traditional paper-based recordkeeping processes, leaving many individuals and organisations with ineffective information resources and increased exposure to access, security and accountability risks. Previously, one of the primary activities of records managers was to maintain paper-based inventories of records as they were created and received within organisations; this focus has shifted to working alongside multiple organisational contexts to understand business processes in order to 
identify where decisions are made and consequently where records are created (Oliver, Evans, Reed, \& Upward, 2010).

Potential challenges specifically relating to managing records in digital formats include issues related to the rapid accumulation of digital artifacts with limited appraisal, the possibilities of human and technical failures (e.g. accidental deletion, back-up failures) and the ease of altering digital records without leaving a trace. In addition, the rate of technological change and lack of integration between systems introduce further potential challenges (Barry, 2010, p. 158). Digital preservation, or permanent access to information and the longevity of digital records, continues to be an issue, which introduces further challenges, including technological obsolescence, lack of awareness and financial sustainability (Adu \& Ngulube, 2017). The application of traditional archival theory and principles, such as original order, appraisal theory and archival bond to digital records can also require significant consideration (O'Meara \& Tuomala, 2012; Zhang, 2012). Appraisal theory and the fundamental purpose of recordkeeping and the role archives was questioned by Findlay $(2013$, p. 8) with regard to the impact of WikiLeaks; “WikiLeaks shows us how archives can be formed and pluralised directly from the affairs that the records document, so serving an extremely powerful purpose in society". Challenges also exist with regard to public records that only exist or are fully functional in a digital environment and without a physical equivalent (Meijer, 2003, p. 16).

The vast majority of New Zealand cultural institutions hold born-digital archival material, that is content created digitally and without an analogue original or equivalent (Moran, 2017, p. 3). Born-digital records may be data in business systems or a line in a database and not stored in an EDRMS, possibly without individual 
metadata $^{1}$ associated with the line items, where the individual data cannot be separated from the business system or database without losing its context (K. Wright, 2014, p. 219). The increasing use of ICTs to create records also changed the way organisations collected, processed and stored individuals' private details, making this information more readily available than ever before and potentially liable to breaches of privacy (Cullen \& Reilly, 2008, p. 61).

During the 1980s and 1990s, the recordkeeping and archival communities acknowledged that the impact of records created digitally required new ways of thinking about managing records (Bearman, 1994; Cook, 1994, 1997). As Cumming (2010, p. 46) reflected it was clear at that time that with increasing creation and use of digital records, the long-term preservation of records as well as the maintenance of their meaning and evidentiality, was dependent on how they were created and managed from the time of their creation. McDonald (1995, p. 70) described the recordkeeping environment of this time as 'the wild frontier': "Office workers can create and send electronic messages and documents to whomever they wish. They can store them according to their own individual needs and then delete them without turning to anyone else for approval." As Lomas (2013, p. 51) described, the majority of research at this time focused on issues surrounding archival concerns of accountability over time, including the role of metadata, definitions and attributes of digital records, and authenticity. As a result of the research conducted during the 1980s and 1990s, researchers developed the records continuum theory and created standardised recordkeeping metadata schemas.

\footnotetext{
${ }^{1}$ Metadata is defined by Archives New Zealand as information that helps people to find, understand, authenticate, trust, use and manage information and records. If information and records have metadata, we know what it is, what it has been used for, and how to use it. Metadata also makes information and records easier to find (Archives New Zealand, 2016b).
} 


\subsubsection{The records continuum theory}

There are two dominant theoretical approaches to records management. The lifecycle theory was defined by the National Archives of the United States of America in the 1930s. This theory is based on the premise that it is possible to divide the life of a record into eight distinct stages, starting with a records management phase consisting of:

1. Creation or receipt of records,

2. Classification of the records in a logical system,

3. Maintenance and use of the records, and

4. Disposition of the records through either destruction or transfer to an archive.

This is then followed by a second, archival phase consisting of:

5. Selection or acquisition of the records by an archive,

6. Description of the records in inventories and finding aids,

7. Preservation of the records or, possibly, the content of the records, and

8. Reference and use of the archives by researchers and scholars (Atherton, 1985, p. 44).

However, limitations of the lifecycle approach include:

- The increasing use of ICTs has meant the stages in the life cycle cannot necessarily be separated; creation is an ongoing process rather than an event 
in time, and the record is likely to be modified and altered a number of times during its period of current use (Atherton, 1985, p. 47).

- Records creation is not the first step in a comprehensive model; system design is the first stage in the development of a recordkeeping system (Tough, 2006).

- Some records may be of value for historical and cultural purposes while simultaneously be of value for practical or administrative purposes (Tough, 2006).

- The lifecycle approach creates a distinction between current recordkeeping and archives management and between the work of records managers and archivists, which does not recognise the interdependence of managing current records and archives management (Atherton, 1985; McKemmish, 1997).

The development of the records continuum theory was an attempt to address some of the limitations of this purely linear, lifecycle approach to records and archival practices and provides a framework for interpreting records and archiving thinking and practice (McKemmish, 2001) (see Figure 2.1). 


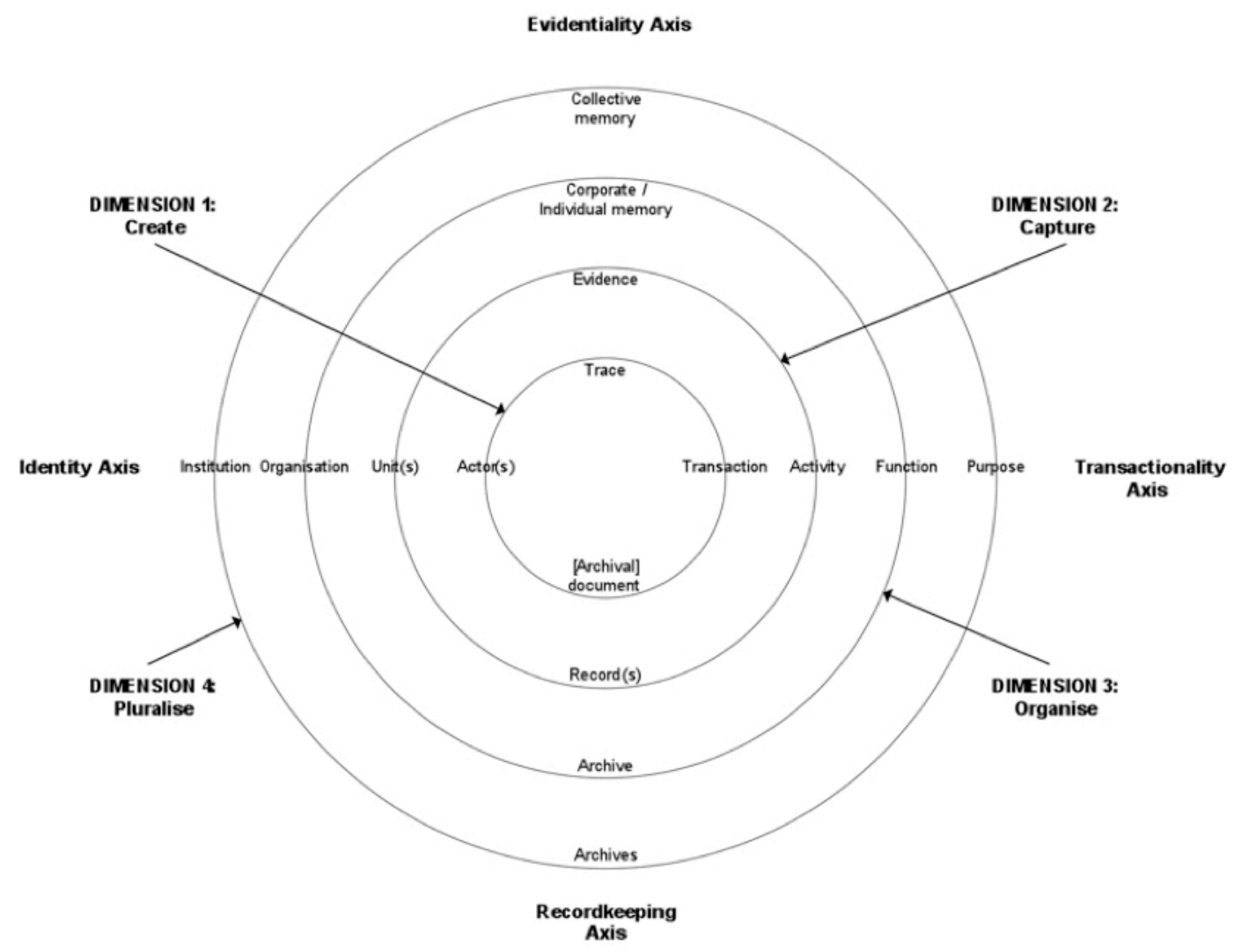

Figure 2.1: The records continuum (Upward, 2000, 2010)

The records continuum is based on a 'create - capture - organise - pluralise' rhythm of processing, reflected in the continuum model as four dimensions. The first dimension occurs when records are created, where the focus is on the importance of individual actions (Upward \& McKemmish, 2006, p. 222). The second dimension, capture, involves the routine storage and linking of records in systems in ways that enable sharing and re-use in the immediate business activities where the transactions are recorded (Upward \& McKemmish, 2006, p. 222). The third dimension, organise, relates to organising records so that others not directly involved in specific business and social processes can access and use what has been created and captured (Upward \& McKemmish, 2006, p. 223). The fourth dimension, pluralise, relates to the broader social environment in which records operate, including the legal and regulatory environment. This dimension also represents the 
capacity of a record to exist beyond the boundaries of a single creating entity (Reed, 2007, p. 20).

In addition to the four dimensions of the records continuum theory, there are four axes:

- The recordkeeping axis, which represents the vehicles for the storage of recorded details about activities or transactions, including the document, the record, the archive and the archives.

- The evidence axis, which consists of the trace of actions, the evidence which records can provide, and their role in corporate and collective memory.

- The transactional axis, which presents the act, activities, functions and purposes of the records.

- The identity axis, which represents the actor, the work unit, the organisation and how the identity of these elements are institutionalised in the broader social context (Upward, 1996, pp. 279-280).

The records continuum theory represented a significant change in recordkeeping and archival thinking.

\subsubsection{Reconceptualising records management}

More recently, recordkeeping informatics builds on the foundation provided by the records continuum. In recognition of the vast changes introduced by advances in ICTs, recordkeeping informatics is a reconceptualised approach to recordkeeping, which incorporates the way recorded information is captured, archived and disseminated as evidence using currently available ICTs (Oliver, Evans, Reed, \& 
Upward, 2009; Oliver et al., 2010; Upward et al., 2013). Recordkeeping informatics aims to provide a framework for tackling recordkeeping challenges in all their technological, organisational and societal complexity (Evans et al., 2014, p. 209).

Recordkeeping informatics has records continuum and recordkeeping metadata as its two foundational building blocks and specifies three key facets of analysis: organisational culture, business process and archival access. The first facet, organisational culture, emphasises the importance to recognise and understand the values, attitudes and behaviours influencing information management in organisations, which ultimately reflects their information culture (Oliver et al., 2010, p. 44). The second facet of analysis is a review of business processes in ways that identify the ongoing use and the adequate capture of recorded information at appropriate points in business processes (Upward et al., 2013, p. 45). The third facet of analysis is access; one of the most challenging aspects of the recordkeeping agenda, exposing the connections between recordkeeping informatics with political and social concerns (Upward et al., 2013, p. 46).

Evans et al. (2014) employed a recordkeeping informatics approach to examine the data, information, records and knowledge management challenges in a research data context. A key finding identified as a result of this approach was the attention on collaboration and community; "the emphasis on the conceptual frame of continuum thinking and metadata, and then the culture, process and access facets of analysis can be a mechanism for bringing recordkeeping and other information management professionals together with management and workers to address complexities through shared explication of problems and translation into solutions" (Evans et al., 2014, p. 220). 
Recordkeeping informatics is an emerging approach, which requires further research and development to address the recordkeeping challenges that exist within complex data, information, knowledge and organisational contexts (Evans et al., 2014, p. 220); however, the focus on organisational culture, business process and archival access underpinned with continuum thinking and metadata provide a relevant and thought-provoking extension of records continuum.

\subsubsection{Personal information management strategies}

Few studies have looked specifically at the ICT-enabled recordkeeping behaviours of front-line public servants; however, research into personal information management provides a theoretical basis for understanding the information behaviours of individuals, in combination with the recordkeeping informatics approach and records continuum theory.

Personal information management is a distinct area of study, which explores how individuals manage the information, including records, documents and data, that they use within their workplace (Boardman \& Sasse, 2004, p. 583). A large part of the early personal information management research focused on tools and the organisation of personal collections, as well as the processes of saving, organising, and retrieving of information; recent personal information management literature has underlined the significance of the contextuality of personal information and its use (Huvila, Eriksen, Häusner, \& Jansson, 2014).

An influential study in personal information management is Tom Malone's 1983 research into how office workers organise documents. Malone (1983) conducted exploratory observations and interviews with ten officer workers and identified two basic personal information management strategies: filing and piling. "Filers" maintained clean desks and did not allow papers to pile up; they arranged their 
papers systematically (e.g. alphabetically or chronologically). In contrast, "pilers" had messy desks cluttered with piles of paper and made few attempts to organise the documents. Since Malone's (1983) influential work, further strategies for the management of personal information have been identified, across different information formats (refer to Table 2.1).

Table 2.1: Selection of salient personal information management strategies

\begin{tabular}{|c|c|c|c|}
\hline Reference & Format & Strategies & Description \\
\hline Mackay (1988) & Email & $\begin{array}{l}\text { Prioritisers } \\
\text { Archivers } \\
\text { Requesters } \\
\text { Responders }\end{array}$ & $\begin{array}{l}\text { Conducted a series of interviews and email } \\
\text { usage analysis to identify three major } \\
\text { forms of work management: } \\
\text { - email as a time management tool, } \\
\text { - email as an information management } \\
\text { - system, and } \\
\text { - email for task management. }\end{array}$ \\
\hline $\begin{array}{l}\text { Whittaker and } \\
\text { Sidner (1996) }\end{array}$ & Email & $\begin{array}{l}\text { Frequent filer } \\
\text { Spring cleaner } \\
\text { No-filer }\end{array}$ & $\begin{array}{l}\text { Identified strategies based on analysis of: } \\
\text { - number of inbox items and total number } \\
\text { of items } \\
\text { - \% of new and old items, } \\
\text { - } \quad \text { inbox conversation threads, } \\
\text { - number of folders and number of } \\
\text { - failed folders, and }\end{array}$ \\
\hline $\begin{array}{l}\text { Abrams, } \\
\text { Baecker, and } \\
\text { Chignell (1998) }\end{array}$ & $\begin{array}{l}\text { Web } \\
\text { bookmarks }\end{array}$ & $\begin{array}{l}\text { No filers } \\
\text { Creation-time } \\
\text { filers } \\
\text { End-of-session } \\
\text { filers } \\
\text { Sporadic filers }\end{array}$ & $\begin{array}{l}\text { Conducted a study of personal Web } \\
\text { information spaces, surveyed } 322 \text { Web } \\
\text { users and analysed the bookmark archives } \\
\text { of } 50 \text { Web users. }\end{array}$ \\
\hline Bälter (2000) & Email & $\begin{array}{l}\text { Frequent filers } \\
\text { Spring cleaners } \\
\text { Folder-less } \\
\text { cleaners } \\
\text { Folder-less } \\
\text { spring cleaner }\end{array}$ & $\begin{array}{l}\text { Used key stroke analysis to identify email } \\
\text { organisational strategies, using the } \\
\text { following variables: } \\
\text { - number of incoming messages, } \\
\text { - number of folders, } \\
\text { - number of searched messages, and } \\
\text { - number of messages in inbox and total } \\
\text { number of messages. }\end{array}$ \\
\hline
\end{tabular}




\begin{tabular}{|c|c|c|c|}
\hline $\begin{array}{l}\text { Gwizdka } \\
\text { (2004) }\end{array}$ & Email & $\begin{array}{l}\text { Cleaners } \\
\text { Keepers }\end{array}$ & $\begin{array}{l}\text { Identified behaviour clusters based on } \\
\text { email variables: } \\
\text { - email interrupts other tasks, } \\
\text { - uses search in email, } \\
\text { - keeps events in email, } \\
\text { - keeps to-dos in email, and } \\
\text { - emails self-reminders. }\end{array}$ \\
\hline $\begin{array}{l}\text { Boardman and } \\
\text { Sasse (2004) }\end{array}$ & $\begin{array}{l}\text { Digital } \\
\text { documents, } \\
\text { email and web } \\
\text { bookmarks }\end{array}$ & $\begin{array}{l}\text { Pro-organising } \\
\text { Organising } \\
\text { neutral }\end{array}$ & $\begin{array}{l}\text { Investigated if participants were } \\
\text { total/frequent filers, extensive filers, } \\
\text { occasional/partial filers or no filers to } \\
\text { determine the level of organising effort. }\end{array}$ \\
\hline $\begin{array}{l}\text { Henderson } \\
(2009)\end{array}$ & $\begin{array}{l}\text { Digital } \\
\text { documents }\end{array}$ & $\begin{array}{l}\text { Filing } \\
\text { Piling } \\
\text { Structuring }\end{array}$ & $\begin{array}{l}\text { Conducted interviews, a survey and file } \\
\text { system snapshot to understand how } \\
\text { people manage their personal document } \\
\text { collections and to develop guidelines for } \\
\text { the development of tools to support } \\
\text { personal document management. }\end{array}$ \\
\hline
\end{tabular}

In addition to describing various strategies outlining how individuals relate to information, Henderson's (2009) investigation into how individuals manage different types of information identified that individuals used different categories of documents and data in the workplace depending on the value they placed on it. These categories are based on three broad classifications: archived, working and ephemeral (Barreau, 2008, p. 308). Archived documents and data have long-term value, but are unrelated to current work and are generally completed (Barreau, 2008, p. 308). Studies have shown that archived documents and data are rarely used (Barreau, 2008, p. 308; Barreau \& Nardi, 1995, p. 42; Boardman \& Sasse, 2004). Working documents and data are frequently used over a period of weeks, months or years, whereas ephemeral documents and data have a relatively short shelf life, but required prominent placement in the workspace so that they are not overlooked (Barreau, 2008, p. 308).

Barreau (1995, p. 327) proposed that personal information management behaviours may be influenced by the hardware and software used by organisations, and the 
personal information management behaviours may impact personal as well as organisational efficiency. Pare (2011) investigated factors that influenced personal information management behaviours and identified seven categories of factors that can contribute to shaping a worker's personal information management system (see Figure 2.2).

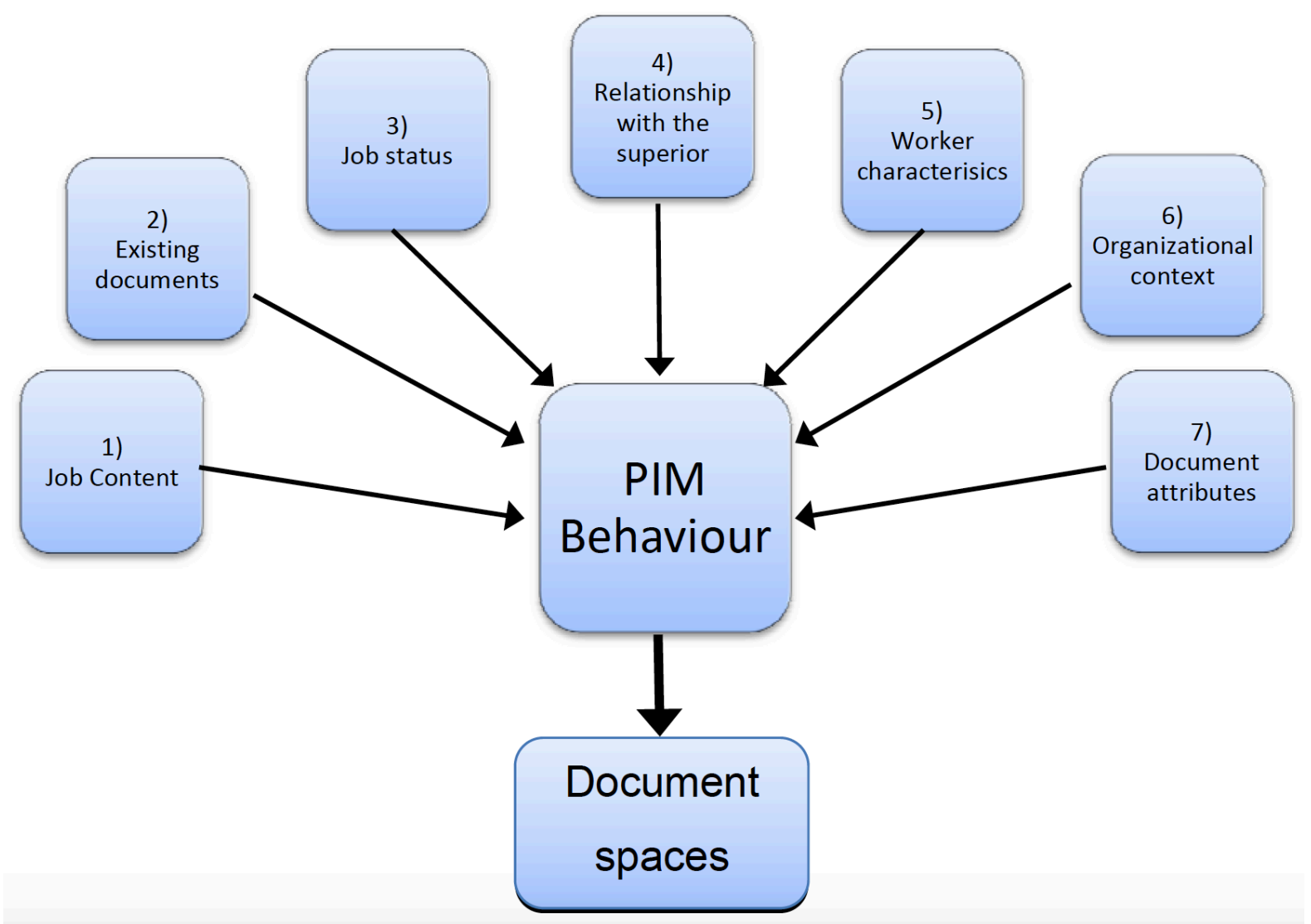

Figure 2.2: A conceptual model for personal information management of office support staff workers (Pare, 2011, p. 143)

While personal information management research is limited in its identification and confirmation of specific influences on information behaviours, in their research both Barreau (1995) and Pare (2011) highlighted that the range of factors are broad, complex and situational. 
Within personal information management research, generally there is limited acknowledgement of the specific requirements relating to recordkeeping within organisations although a small number of studies acknowledge recordkeeping. Winget, Chang and Tibbo (2007) conducted a three-year project examining the desktop computing environments and behaviours of staff at two major US universities, in an attempt to understand how individuals interacted with digital records. The research attempted to address the 'competing concerns' between users applying personal information management strategies and the requirements of records managers and archivists. Winget et al. (2007) found that user concerns revolved around the volume of files with which they are forced to contend, leading to wasted time, improperly deleted files, confusion, and general dissatisfaction with the technical environment. On the other hand, records managers and archivists were concerned with the preservation of digital materials and organisational issues, often at odds with users' daily practice. As Winget et al. (2007) outlined personal information management strategies made life easier for users as they provided a means for coping with the management of digital records, but they often did not take into account the archival need to preserve the context in which a digital record was created or received.

The major difference between recordkeeping and personal information management relates to the perspectives of the two disciplines. Huvila et al. (2014) explored how contextual aspects, conceptualised as axes of the record continuum, influenced the practices of capturing, organising and pluralising within a personal information management context. A further difference between the records continuum model and the specific context of personal information management outlined by Huvila et al. (2014) relates to the individual versus collective perspectives of the models. Recordkeeping informatics within the broader context of the workplace is essentially 
a societal model whereas personal information management is focused on individuals (Huvila et al., 2014).

\subsubsection{Understanding the recordkeeping behaviours of individuals}

While records continuum and recordkeeping informatics provide a basis for understanding the recordkeeping context of individuals and personal information management strategies provide an understanding of the behaviours of individuals in managing data and documents, neither specifically address the unique context of how individuals create, manage and use records within public agencies. As mentioned previously, there is limited research into the recordkeeping behaviours of individuals within ICT-enabled organisations from the perspective of the front-line public servants as the recordkeepers; some scholars suggest this lack of research and organisational focus is, in part, related to the invisible nature of recordkeeping.

Yakel (1997, p. 21) emphasised the opportunity offered by studying recordkeeping processes in situ to demonstrate how recordkeeping is situated within the context of interrelated processes and to illustrate the limits of records and recordkeeping as actual representation of events and processes. Yakel (1997, p. 2) explained "recordkeeping is ubiquitous. However, its routineness and the fact that it is often embedded in large processes, make recordkeeping easy to overlook. In order to fully understand the context of recordkeeping activities, a broad examination of organisational context is necessary".

Shankar (2004, p. 368) argued that recordkeeping processes in organisations eventually become transparently embedded into other internal organisational processes and systems, and if successful the recordkeeping infrastructure becomes invisible and 'natural' to those who use it, unless it breaks down, or is the object of study. 
With the ubiquitous, yet invisible, nature of recordkeeping within the organisation in mind, understanding recordkeeping from the perspective of individuals as recordkeepers provides a meaningful lens to better understand organisational and broader societal recordkeeping contexts. Meijer (2001, p. 264) explained that the increasing use of ICTs emphasised individual autonomy, shifting the control over the creation and management of information from centralised organisational control to individual control. In many organisations, for example, individuals manage email accounts and messages, decide which messages are saved or deleted and thus control the creation and capture of records.

Meijer (2001, p. 264) described how compared to organisations, individuals are focused on their current work activities and tend to be less aware of long-term, broader, interests, impacting the potential organisational memory. Similarly, Atherton (1985, p. 49) argued that records are created during a transaction or as evidence of a transaction to serve an immediate operational purpose. A report reviewing the United Kingdom government's approach to managing digital records (Allan, 2015) surmised that when departments adopted an EDRMS or a structured shared drive approach, it was the responsibility of individuals to identify records that were significant and required for preservation. Individuals were then expected to save those records in the EDRMS or corporate file plan. While some staff did this scrupulously, many found it an unwanted burden as the act of saving a record into an EDRMS required staff to complete a range of metadata to provide the details to identify the record and its content, and it was often easier for staff to find and retrieve records they had stored on their personal drives (Allan, 2015, pp. 4-5). The report identified two components that influenced this situation: firstly, organisation culture and attitudes towards information, reflecting a lack of awareness and understanding of the importance of good recordkeeping; and secondly technology, 
reflecting that the ICTs made it burdensome for staff to save records (Allan, 2015, p. $5)$.

This suggestion that ICTs make it burdensome for staff to save records was also explored by S.G Shaw, Pedersen, Cooley and Callingham (2013) who investigated how pre-service teachers used an EDRMS to collect, record, interpret and use classroom data on their students during professional experience. The research identified a range of themes that prevented or limited the ability of the participants to use the EDRMS in ways that aligned with their intentions, including:

- accessibility (including the lack of portability of the EDRMS, technical issues, issues with trust, and inconvenience),

- prioritisation (in particular, that the pre-service teachers prioritised other events in the classroom over recordkeeping),

- duplication (as the majority of pre-service teachers made hand-written notes and then, at a convenient time for them, duplicated the details into digital form to enter in ICTs, making this a time-consuming process) and

- relationships (whereby the pre-teachers found it difficult to keep records on their students before a relationship with the students was established) (S. G. Shaw et al., 2013, p. 79).

Recognising that the success of EDRMS implementations in general, as measured by acceptance and use by individuals, has been mixed, Lewellen (2015) conducted New Zealand-based research that sought to determine the factors that influenced staff members' intentions to use an EDRMS. Lewellen (2015, p. 221) identified that the three most important constructs influencing whether staff members used the 
EDRMS were in order of significance, the perceived value of the records, effort expectancy and social influence. Lewellen (2015, p. 223) suggested that emphasising the ease of use of the system to staff, rather than potential opportunities to create gains in job performance would motivate staff to use the EDRMS; likewise, focusing on the importance of contextual metadata and emphasising the value of records would increase the perceived benefit of using the EDRMS.

In research designed to understand factors impacting the use of an EDRMS at a single agency in Botswana, Mosweu, Bwalya and Mutshewa (2016, p. 59) identified that attitudes to computers, including computer anxiety, incompatibility of the EDRMS with current work habits, staff practices and beliefs, as well as the perceived complexity of the system negatively influenced the adoption of the EDRMS.

Investigating 'the messiness of everyday work life' from the perspective of the genre of the documentary texts and examining the interactions in which staff routinely engaged as they used ICTs to accomplish their work activities, Spinuzzi $(2003$, p. 4) argued places user driven innovations at centre stage. Spinuzzi (2003) advocated examining the approaches that individuals take when working with information in ICT-enabled environments drawing on genre theory and activity theory; whereby genre theory provided the unit of analysis and activity theory provided a foundation for studying the different levels of activity within an organisation. Spinuzzi (2003, p. 58) argued that a genre tracing approach to information design valued the wide array of unofficial innovations that individuals used to customise ICTs for their own uses.

Within the context of this study, it is this complex and indistinguishable relationship between public servants and the recorded information they work with on a daily basis, the systems they use and the work behaviours they employ that forms the 
crux of the study; acknowledging that both the materiality of organisational life and the unofficial innovations that individuals use assist in developing an understanding the recordkeeping behaviours. In summary, while there is limited empirical research that builds an understanding of the recordkeeping behaviours of public servants, partly as a result of the invisible nature of records within business processes, a combination of recordkeeping informatics drawing on records continuum, and personal information management provide part of a framework to better understand these behaviours. Each of these underscore the context that the behaviours occur within; in particular, the public sector, the public agencies and the specific work unit or team.

\subsection{Working in the public sector}

\subsubsection{The nature of recordkeeping in the public sector}

As the United Kingdom's Cabinet Office (2017, p. 7) explained it was during the 1990s that public service activity began to transition away from paper. Prior to this, in the most part, public offices conducted their business using physical records and had the supporting recordkeeping systems, including mail systems, typing pools, filing cabinets, and records clerks to manage paper workflows. The United Kingdom's Cabinet Office (2017, p. 7) described the use of physical records within government departments in the paper-based environment:

\footnotetext{
Paper ways of working made information management reasonably straightforward: records were a tangible asset which physically resided in one place only. When a file's short-term business value came to an end the author or owner would take a view on its long term value and either destroy it or transfer it to the relevant archive within the department. At the thirty-year stage $\mathrm{KIM}^{2}$ professionals would select what needed to go to The National Archives (destroying what was now superfluous) and then review selected documents before either storing them locally in a secure holding (if they
}

\footnotetext{
${ }^{2}$ Knowledge and Information (KIM) professionals.
} 
continued to be highly sensitive) or transferring them to The National Archives open or closed, if of lesser sensitivity.

With the introduction of ICTs and digital ways of working, public servants conducted daily business digitally and government information was increasingly created, managed and used in digital formats. The responsibility for day-to-day recordkeeping fell largely to public servants (Cabinet Office Digital Records and Information Management Team, 2017, p. 5). Paper memos became emails, reports were created in word processors, spreadsheets and presentation software, and internal paper mail services were reduced. Over time, as the United Kingdom's Cabinet Office (2017, p. 8) described, there was a reduction in information management activity at a time when the amount of information generated by government dramatically increased. Some public servants realised that saving digital information was important but they did this themselves, in their own way, rather than using agency systems. Some agencies forced staff to manage information in particular ways by closely restricting where and how much information could be saved (e.g. email inbox size) but others had few restrictions meaning that information accumulated in an unstructured way and became fragmented on personal drives, shared drives and email folders.

Changes to the way governments operate introduced through the increasing use of ICTs have also changed the way public agencies interact with citizens and how individuals' personal information is collected, processed and stored (Cullen \& Reilly, 2008, p. 61). From a citizen's point of view, manual form filling or presenting physical official identity documents and other forms of identity have become increasingly superfluous; while to the public servant, the management and use of large collections of physical records stored in a personal file related to a particular citizen increasingly belong to the past (Lips, Taylor, \& Organ, 2009, p. 851). In ICT- 
enabled government agencies, public servants carry out much of their daily work using computers, rather than using physical records, which has also allowed public servants to become increasingly mobile in their work practices.

While a still developing initiative, the use of social media offers governments and public agencies extensive and new opportunities to engage with citizens (Omar, Stockdale, \& Scheepers, 2014, p. 666). As Meijer and Torenvlied (2016, p. 143) explored in empirical research investigating the use of microblogging by Dutch police departments; microblogging provides a direct channel for many police officers to communicate externally in ways that are not directly controlled by central communications offices. However, Meijer and Torenvlied (2016, p. 157) reinforced that the transformative nature of social media to government communication should not be exaggerated as much police communication was still taking place through traditional communication methods, for example face-to-face contacts, telephone conversations, email, and written letters.

In their investigation of local governments implementing social media initiatives within local government organisations in the State of Victoria, Australia, Omar et al. (2014, p. 673) found that governments are limited in their use of social media tools, often with no clear aim to interact with the citizens, and that agencies are more motivated by the desire to have a presence in and experiment with social media. This investigation identified potential issues of social media use by local government, including challenges controlling content, uncertainty, fear of risk, lack of knowledge and resources, technology ownership, and lack of trust (Omar et al., 2014, pp. 669-672). The ability to capture and control social media conversations as records was mentioned by interview participants, although not expanded upon in the research (Omar et al., 2014, p. 670). 
The relationship that citizens have with government is a unique one, particularly when it comes to sharing information. In many situations, requests from government organisations for citizen's personal information are mandatory; in contrast when individuals exchange personal information with private organisations they are able to decide the organisation and, to some extent, in what ways they provide their personal details (Cullen \& Reilly, 2008, p. 64). Adding to this unique relationship is the sensitive nature of the personal information that citizens provide to government organisations. For example, citizen's financial data provides an important picture of who they are; financial data reveals financial status, where people work, the nature of their occupation, as well as details on a person's health, marital status, lifestyle and hobbies (Sharman, 2009, p. 719).

Historically in public service provision, identification of citizens was undertaken through completion of a paper-based form, often coupled with the submission of physical identification documents to the inspecting public official; resulting in public offices becoming vast repositories of physical records (Lips, Taylor, et al., 2009, p. 835). However, the introduction and use of ICTs in public service relationships has had a substantial impact on informational relationships between citizens and government agencies (Lips, Taylor, et al., 2009, p. 850).

Research into the online behaviours of New Zealanders found that 68 percent of those involved in the research had transacted online with a government agency in a 12 month period (Lips, Eppel, Sim, Barlow, \& Lofgren, 2014, p. 44). This research found that in general, people were quite private in online transactions with government, with the large majority restricting the personal information they share online. The most frequently provided types of personal information in government online relationships were name (93\%), email address (92\%), home address (88\%), and mobile phone number (73\%); IRD number (73\%) and financial details (58\%), 
such as credit card or bank details, were also frequently shared with government online (Lips et al., 2014, p. 44).

This trend of increasing access of government services online was also identified in a 2012 report on online engagement with the New Zealand government, which suggested a gradual yet steadily growing confidence in accessing government agencies and services online (Gibson, Crothers, Smith, Aguirre, \& Bell, 2012, p. 14). Research conducted in 2013 by the World Internet Project on New Zealander's usage of and attitudes towards the Internet also identified New Zealanders were engaging with government online. Of those internet users who responded to the survey, the majority $(59 \%)$ indicated they had used government or council services that were delivered online, $47 \%$ had logged in to secure areas on government or council websites and $51 \%$ had gone online to pay taxes, a fine, or a licence in that past year (Gibson, Miller, Smith, Bell, \& Crothers, 2013, p. 14).

There are numerous impacts as a result of the various personal information collection activities conducted by government organisations using ICTs, including:

- Personal information can flow freely and in ways that are difficult to trace, compared to personal information in face-to-face and paper-based transactions within the confines of a physical locale and relatively closed networks.

- Personal information can be copied and stored at almost no expense.

- An increased merging of previously compartmentalised identity personal information on the citizen. 
- Transactions become information dependent, with current identification systems relying on the confirmation of an individual's details.

- Transactional histories become more detailed and easily available to many.

- Trust depends on transactional history reports rather than on personal recognition.

- An increased blurring of lines between public and private places makes citizen personal information more publicly available (Lips, Taylor, et al., 2009, p. 837).

This changing environment has broad implications for the relationship between citizens and government, particularly when it comes to citizens' trust of the way public offices manage their personal information. An individual's ability to assess the trustworthiness of an organisation, particularly a public agency which is managing one's personal information, is related to expectations and knowledge of that organisation, including the intentions and competence of individuals within that organisation (Cullen \& Reilly, 2008, p. 63).

Research into cross-government information sharing in New Zealand found that the public servants involved were aware of their role to protect personal information. Privacy values were embedded in the way that public servants worked; for instance, public servants shared information on a 'need to know' basis and used signed consent forms as authorisation to share information with other agencies (Lips, O'Neill, \& Eppel, 2009, p. 81); thus reinforcing the changing nature of recordkeeping within the public sector and the impact of ICTs on how public servants work.

\subsubsection{The public sector and the public servant}


There is much research exploring differences between private and public sector working environments for employees. Baarspul and Wilderom (2011, p. 968) explored the proposition that significant organisational differences exist between private and public sectors and that these differences influenced the behaviours of individual employees, which led to their investigation of whether employees behaved differently in public and private sector organisations. The review of twentyeight research papers identified only one significant difference: public servants displayed a higher level of community-service motivation than private sector employees (Baarspul \& Wilderom, 2011, p. 987).

Distinctions between private and public sector employees were also explored by Rohr (1988), who explained that public sector ethics played a central role in guiding the decision-making of public servants during their daily working lives, distinguishing them from their private sector equivalents. Rohr (1988) defined public sector ethics as the public servants' ability to exercise their discretionary powers in a responsible manner and to show sound judgement in their decisions. DeLeon (2003, pp. 569-570) further discussed the influence of public sector ethics on the decisionmaking of public servants, explaining that as the work of public administrators could not be closely and constantly monitored, and in many ways the public sector sought empowered, creative and flexible employees, public servants should be granted the freedom to make decisions based on the public interest, constrained by their ethical responsibilities.

The boundary role that front-line public servants occupy in mediating between the public sector and members of the public has been a longstanding and topical theme in the public sector literature (Bjerregaard \& Klitmoller, 2010, p. 421; Vinzant \& Crothers, 1996, p. 457). Bjerregaard and Klitmoller (2010, p. 422) acknowledged front-line work was challenging, particularly as these staff members were under 
pressure to process an increasing number of citizens while there were scarce human resources, time and norms informing the front-line staff's strategies of handling perceived problems.

Within the public agencies, an underlying tension exists between the need for standard treatment of citizens and the need to consider the unique circumstances of each citizen (Scott, 1997, p. 53). The literature on front-line public servants has identified a broad variety of factors that are claimed to explain front-line decisionmaking, and while much empirical research has been conducted to examine the effects of these factors, the findings from these studies have reached mixed conclusions, reflecting the complexity of the front-line public servants' environment (Loyens \& Maesschalck, 2010, p. 72).

Scott $(1997$, p. 39) identified three broad sets of factors that influence decisionmaking of individuals in public agencies: characteristics of the client, organisational characteristics, and attributes of the service provider. Furthermore, on the basis of experimental qualitative research, $\operatorname{Scott}(1997$, p. 52) found that the organisational control factor played the most influential role, followed by client attributes, and lastly, individual decision-maker attributes. Vinzant and Crothers (1996) expanded this set of factors to include extra-organisational factors, for example the broader community, laws and regulations, the media, other service agencies, and general situational variables.

This, and other, research provided Loyens and Maesschalck (2010) with the basis for theorising the factors that influence front-line workers' decision-making behaviours, based on the following four categories:

- Individual decision-maker characteristics, for example, professional norms, workers' beliefs, and moral values of front-line officers. 
- Organisational characteristics, including the organisation's internal structure, workload pressure, rules and constraints, organisational culture, co-workers and supervisors.

- Client attributes, for example perceived level of need of the client.

- The broader community, including laws and regulations, the media and other service agencies.

\subsubsection{Front-line public servants in ICT-enabled agencies}

In 1980, Michael Lipsky theorised that public servants who interacted directly with citizens had a degree of policy discretion available to them in the execution of their work, describing these front-line public servants as 'street-level bureaucrats' (Lipsky, 1980, p. 3).

Keiser (2010, p. 254) explained that a defining characteristic of street-level bureaucracy theory was the importance of face-to-face interactions between clients and front-line public servants. However, as services and interactions between public servants and citizens are increasingly offered through ICTs and based on egovernment services, these interactions lack a face-to-face component.

Buffat (2015, p. 151) categorised existing literature on the impacts of ICTs on frontline staff and their discretionary abilities as either curtailing arguments, whereby street-level discretion decreases or disappears; or enabling arguments, where research highlighted more nuanced effects of ICTs.

Arguing that ICTs curtail front-line public servants decision-making, Snellen (1998, p. 501) suggested that the work situation of front-line public servants changed rapidly as a consequence of increased automation of decisions introduced through 
the use of ICTs. Snellen (1998, p. 503) stated that it is as a result of ICTs that frontline public servants lost their intermediary position between internal organisational procedures and the circumstances and wishes of clients. Bovens and Zouridis (2002, p. 180) also suggested that the discretion of front-line public servants reduced with the increased use of ICTs and e-government initiatives, proposing that street-level bureaucrats were increasingly being replaced with a network of screen-level bureaucracies. In screen-level bureaucracies, the decision-making process was routinised, and contacts with members of the public always ran through or were in the presence of a computer screen. Within a system-level bureaucracy as proposed by Bovens and Zouridis (2002, p. 180), individual case managers no longer exist as they are replaced by ICTs and process designers, large parts of the business process can be outsourced and client data is exchanged seamlessly between agencies. Bovens and Zouridis (2002, p. 181) proposed this would gradually change beyond screenlevel bureaucracies into a system-level bureaucracy, where legal frameworks were converted into concrete algorithms, decision trees, and modules (see Table 2.2 for a comparison of characteristics of these three forms of front-line bureaucracy).

Table 2.2: Characteristics of street-, screen- and system-level bureaucracies (Bovens \& Zouridis, 2002, p. 180; Reddick, Abdelsalam, \& Elkadi, 2011, p. 393)

\begin{tabular}{|c|l|l|l|}
\hline Role of ICTs & \multicolumn{1}{|c|}{$\begin{array}{c}\text { Street-level } \\
\text { bureaucracy }\end{array}$} & \multicolumn{1}{|c|}{$\begin{array}{c}\text { Screen-level } \\
\text { bureaucracy }\end{array}$} & \multicolumn{1}{|c|}{$\begin{array}{c}\text { System-level } \\
\text { bureaucracy }\end{array}$} \\
\hline Function of ICTs & Supportive & Leading & Decisive \\
\hline $\begin{array}{c}\text { Human interference } \\
\text { with individual case }\end{array}$ & Full contact & Case assessment & $\begin{array}{l}\text { Execution, control } \\
\text { and external } \\
\text { communication }\end{array}$ \\
\hline $\begin{array}{c}\text { Organisational } \\
\text { structure }\end{array}$ & Case managers & Production managers & Systems designers \\
\hline
\end{tabular}




\begin{tabular}{|c|l|l|l|}
\hline $\begin{array}{c}\text { Organisational } \\
\text { boundaries }\end{array}$ & $\begin{array}{l}\text { Strict boundaries and } \\
\text { separation } \\
\text { with other agencies }\end{array}$ & $\begin{array}{l}\text { Strict, both within } \\
\text { and between agencies }\end{array}$ & $\begin{array}{l}\text { Fluid boundaries } \\
\text { within and } \\
\text { between agencies }\end{array}$ \\
\hline Legal regime & Ample discretion & Little discretion & No discretion \\
\hline
\end{tabular}

However, Buffat (2015, p. 154) was critical of the idea that front-line public servant decision-making would disappear with use of ICTs and outlined four weaknesses in relation to the curtailing argument:

- The use of a narrow definition of discretion, particularly by Snellen (1998, p. 500), who defined it as the public servant' ability to manipulate information streams.

- The typology is most suitable for very specific street-level organisations. As Bovens and Zouridis (2002, p. 184) noted, the progression to system-level bureaucracy is only relevant for those public servants that are involved in the routine handling of large amounts of formal transactions, for example, by teachers, health workers, or police officers.

- There has been insufficient interest in the concrete uses of ICTs by front-line public servants and by citizens.

- The impact of ICTs may be overstated, for example technologies arrive and front-line discretion decreases or vanishes.

The 'curtailment argument' was also challenged by other research, which considered new technologies as only one contextual factor shaping front-line discretion (Buffat, 2015 , p. 154). Schuppan $(2015$, p. 248$)$ argued that a major critique that could be levelled against the work of Snellen (1998) and Bovens and Zouridis (2002) was that 
they concentrated solely on ICTs, neglecting other variables in the context of ICTs usage, such as the nature of the task itself (e.g. standardised or non-standardised work), the organisational environment (e.g. stable or dynamic) and the organisational form of work (e.g. networked or hierarchical).

Jorna and Wagenaar (2007) studied two subsidy allocation processes in the Netherlands and demonstrated that while ICTs provided increased managerial control over formal aspects of the daily organisational life, they did not capture informal dimensions of the decisions made by front-line staff. In this way, Jorna and Wagenaar (2007, p. 210) argued that ICTs, rather than decreasing the discretion of front-line public servants, obscured the informal discretionary behaviours of public servants.

Reddick, Abdelsalam and Elkadi (2011) conducted survey-based research that investigated the influence of e-government effectiveness on administrative discretion in local governments in Egypt. They found that ICTs removed the frontline public servant from of the operational processes and as a consequence reduced some elements of the discretion associated with face-to-face contact between public servants and citizens. They also found that collaboration and organisational change were influencing factors on the administrative discretion of front-line staff; however, top management support, information security, size of government and citizens' demand for e-government did not influence the discretion of front-line public servants.

In empirical research into a German government call centre, Schuppan (2015, p. 260) argued that "ICT expands the scope of action of street-level bureaucrats - or, in other words, extends their discretion, their function as policymakers and their particular craftsmanship in fulfilling their tasks - due to the uncertainties emerging in the new 
interaction mode with citizens." As far as system-level bureaucrats were concerned, Schuppan (2015, p. 260) did not observe any significant shift of the kind that Bovens and Zouridis (2002) described. Schuppan (2015, p. 258) identified that the work of public service call centre staff was so diverse and uncertain, and the interaction with citizens was situation-dependent; consequently, the public servants were continuously required to improvise in their decisions about the adequacy of the information they delivered.

Hansen, Lundberg and Syltevik (2016, p. 18) also empirically tested Bovens and Zouridis' (2002) proposition of screen- and system-level bureaucracy and did not encounter empirical evidence for system-level bureaucracy in their analysis of the ICTs implementation within the Norwegian Welfare and Labour Organization. Hansen et al. (2016, p. 18) confirmed that Norwegian Welfare and Labour Organization was a street-level bureaucracy that had some features of a screen-level bureaucracy for some groups; however, at the time of their research, ICTs had not replaced face-to-face encounters and had not altered the decision-making discretion of front-line public servants. The most typical interaction pattern between front-line public servants and citizens identified was to combine ICTs with face-to-face contact, as many citizens faced complex problems that did not fit into predefined algorithms (Hansen et al., 2016, p. 18).

There is general confirmation by researchers that the work of public servants has changed as a result of the increased use of ICTs, and that there are multiple factors influencing their decision-making behaviours of front-line public servants. Schuppan (2015, p. 243) argued that the introduction of e-government and ICTs in public agencies changed the structure and dynamics of how public work was organised. In ICT-enabled agencies, front-line public servants confronted a wider variety of citizens' requests and they made decisions about the delivery of larger 
amounts of information and multiple information sources (Schuppan, 2015, p. 243). However, recent empirical research argues that there is little evidence that interactions with citizens are conducted solely online or that the actions and decisions of front-line public servants are made by ICTs (Hansen et al., 2016; Schuppan, 2015).

\subsection{Recordkeeping behaviours in the public sector}

\subsubsection{ICTs, organisations and behaviours}

The place of technology in human activity systems in an organisational context has been examined through many different theoretical approaches, although as Orlikowski (2007, p. 1435) described much of this organisational research overlooked the ways in which organising is bound up with the material forms and spaces through which humans act and interact. Furthermore, most information systems research has taken a limited theoretical focus on the digital artifact (Anderson \& Robey, 2017, p. 100).

In developing an understanding of the nature of organisations, Orlikowski proposed researchers draw on ways of engaging with the everyday materiality of organisational life that recognises the inextricable relationships between the social and the material; "there is no social that is not also material, and no material that is not also social" (Orlikowski, 2007, p. 1437). As Orlikowski (2006, p. 460) explained, "everyday practices and the knowing generated as a result is deeply bound up in the material forms, artifacts, spaces, and infrastructures through which humans act".

Zuboff (1988) sought to understand how ICTs affect both individual and collective organisational behaviour as she investigated the impacts of technological changes within organisations at times when, for many of those people being studied, it was their first time they were expected to work with ICTs, and where ICTs were 
implemented in ways that fundamentally changed how people were required to complete their daily work. Furthermore, as Zuboff $(1988$, p. 10) described "in the office environment, the combination of on-line transaction systems, information systems, and communications systems creates a vast information presence that now includes data formerly stored in people's heads, in face-to-face conversations, in metal file drawers, and on widely dispersed pieces of paper". As well as automating many of the tasks previously completed by people, Zuboff $(1988$, p. 9) described how ICTs simultaneously generated new forms of information about the underlying productive and administrative processes through which an organisation accomplishes its work, providing a deeper level of transparency to activities that had been either partially or completely opaque. Ultimately, Zuboff (1988, p. 395) described an organisational working environment where "jobs are comprehensive, tasks are abstractions that depend upon insight and synthesis, and power is a roving force that comes to rest as dictated by function and need". While organisations may not (yet) fully function as Zuboff (1988) described, the nature of work conducted by staff has significantly shifted as activities are automated, as have the nature of the organisations with the introduction of ICTs.

Much focus of research on use of ICTs has been on how users come to accept and use a technology, including through technology acceptance, which has led to the development of the Technology Acceptance Model (Davis, 1986), the Technology Acceptance Model 2 (Venkatesh, \& Davis, 2000), the Unified Theory of Acceptance and Use of Technology (UTAUT) model (Venkatesh, Morris, Davis, \& Davis, 2003, p. 447), as well as the Technology Acceptance Model 3 (Venkatesh \& Bala, 2008, p. 280). Conceptually, technology acceptance is based on the observation that an actual behaviour (e.g., system use or 'tool use') is highly correlated with the intention to perform a behaviour; in addition, the intention to perform the behaviour is determined by the user's perception of the technology's usefulness and its ease of 
use (Lewellen, 2015, p. 36). These two constructs form the basis for one's attitude toward use, which together influence one's intention to use and, ultimately, use.

However, as Lewellen (2015, p. 44) pointed out while the TAM constructs target an individual's perceptions toward a particular technology, within the context of recordkeeping, EDRMS are necessarily used within an organisation; consequently, these systems occur within a context where environmental factors, particularly interactions with other individuals and cultural norms, can affect an individual's perceptions of the technology. Lee (2014) described TAM and UTAUT as 'incomplete models' because they failed to take into account the social structures in which the individual operated. Furthermore, TAM constructs focused primarily on perceived personal benefits and failed to consider organisational benefits, except through the lens of the individual (Hardgrave \& Johnson, 2003). Lewellen (2015, pp. 223-224) argued that technology acceptance did not work as expected in a recordkeeping context; suggesting that recordkeeping systems in public sector organisations have a unique set of factors influencing use. For example, Lewellen (2015, pp. 223-224) identified that those individuals who placed a high value on records were more likely to be interested in managing those records in the organisational recordkeeping system, aligning with the individual decision-making characteristics as presented by Loyens and Maesschalck (2010).

In longitudinal research into an electronic health record system implementation in a multi-site medical group, Strong et al. (2014) applied the concept of affordances to understand user behaviours and ICT-associated organisational change. Originally from the field of ecological psychology, affordance theory is used as a lens for theorising ICT-enabled change. In the development of the affordance-based theory of ICT-associated organisational change, Strong et al. (2014, p. 53) considered the materiality of the digital artifact, the non-deterministic process by which ICTs lead to 
organisational effects, the multi-level nature of ICT-associated change processes, and the intentionality of managers and individuals as agents of change. In addition, Strong et al. (2014, p. 72) identified that the abilities and preferences of the individual, the features of the system, and the characteristics of the work environment both supported and restricted an individual's affordance actualisation.

Also drawing on affordance theory and acknowledging the organisational context in examination of individual behaviours, in case study research, Anderson and Robey (2017) evaluated the use of an electronic medical records system to understand the nature of digital artifacts and their relationships to technology, staff and work practices. Anderson and Robey (2017) suggested that affordances can be realised in a variety of ways by staff seeking to attain specific goals; however, the findings reinforced that staff members' goals and perceptions must be understood in relation to specific technology features, technical abilities, and the wider organisational context in which the technologies are used (Anderson \& Robey, 2017, p. 111).

\subsubsection{Information culture and recordkeeping}

While complex to define, information culture is a theoretical construct used to explore the role of norms, attitudes and the way that organisations value information (Sundqvist \& Svärd, 2016, p. 9). Information culture is closely intertwined with the broader cultural constructs within organisations (Choo, Bergeron, Detlor, \& Heaton, 2008; Douglas, 2010; Oliver, 2011; Svärd, 2014).

Research on information culture has tended to focus on three areas: information culture and business performance (Ginmen, 1987; Widén-Wulff, 2000); information culture and information systems (Leidner, 1988; Owens, Wilson, \& Abell, 1995; Travica, 2008); and information culture in individual organisations (Choo et al., 2008; Curry \& Moore, 2003; Douglas, 2010). However, the relationship between 
information culture and records management or recordkeeping has rarely been explicitly discussed (Sundqvist \& Svärd, 2016, p. 12). Furthermore, there is limited evidence of the study of information culture within the public sector (Douglas, 2010).

From 2007 to 2010, a significant research project was conducted in the United Kingdom, which explored records management from three perspectives (i) people, including vision, awareness, culture, drivers and barriers; (ii) processes, including working practices, procedures, policies and standards; and (iii) systems/technology, in terms of the design principles for delivering effective recordkeeping (Childs, McLeod, \& Hardiman, 2011). Participants highlighted that people issues concerned all three of these facets; one of the significant findings from this study was that people issues were predominant, fundamental and challenging, because they concerned culture, philosophical attitudes, awareness of records management and electronic recordkeeping issues, preferences, knowledge and skills (Childs et al., 2011, p. 74).

Drawing on information culture assessment tools (Curry \& Moore, 2003), T. Wright (2013) explored the relationship between records management training provided to staff, staff self-perceptions of records management competencies and compliance with a formal records management programme within a regulated, government environment. The categories that T. Wright (2013, p. 19) employed to assess the information culture included strategy and objectives, information environment, professional associations, information systems, relationships, and communications. She found that there was a potential relationship between formal training delivered to staff and staff's perceptions of their records management competencies; however, as the records management training strategy was informal, T. Wright (2013, p. 14) found it was difficult to determine the influence of the training programme on the organisation's information culture. 
In 2005, Oliver investigated information culture within three distance education universities in Australia, Germany and Hong Kong, where information management processes encompassed recordkeeping and library activities as well as publishing functions. Oliver (2005, p. 287) identified that geography, economics, language, and politics influenced the information culture within organisations, thus extending the concept of information culture to include the concept of national culture. Oliver (2005, p. 287) also suggested that occupational influences and corporate culture had some influence on the information culture within the case studies she conducted.

Oliver extended this research to develop a framework for the analysis of information culture within organisations, comprising of three levels (Oliver, 2011; Oliver \& Foscarini, 2014; Oliver, Foscarini, Sinclair, Nicholls, \& Loriente, 2018). The first level deals with the fundamental layer of an organisation's culture; that is, the factors that are very hard to change, including:

- the value accorded to records, or respect for information as evidence;

- $\quad$ preferences for different communication media and formats, as well as preferences with regard to sharing information;

- language requirements; and

- regional technological infrastructure (Oliver \& Foscarini, 2014, p. 18).

The second level addresses employee skills, knowledge and experience related to information management. This is located in the middle of the triangle because training and development will take into account those fundamental influences at the bottom (Oliver \& Foscarini, 2014, p. 18). The third level concerns information governance and trust in organisation information systems. These factors are 
significant for organisational recordkeeping and are the most susceptible to change (Oliver \& Foscarini, 2014, pp. 18-19).

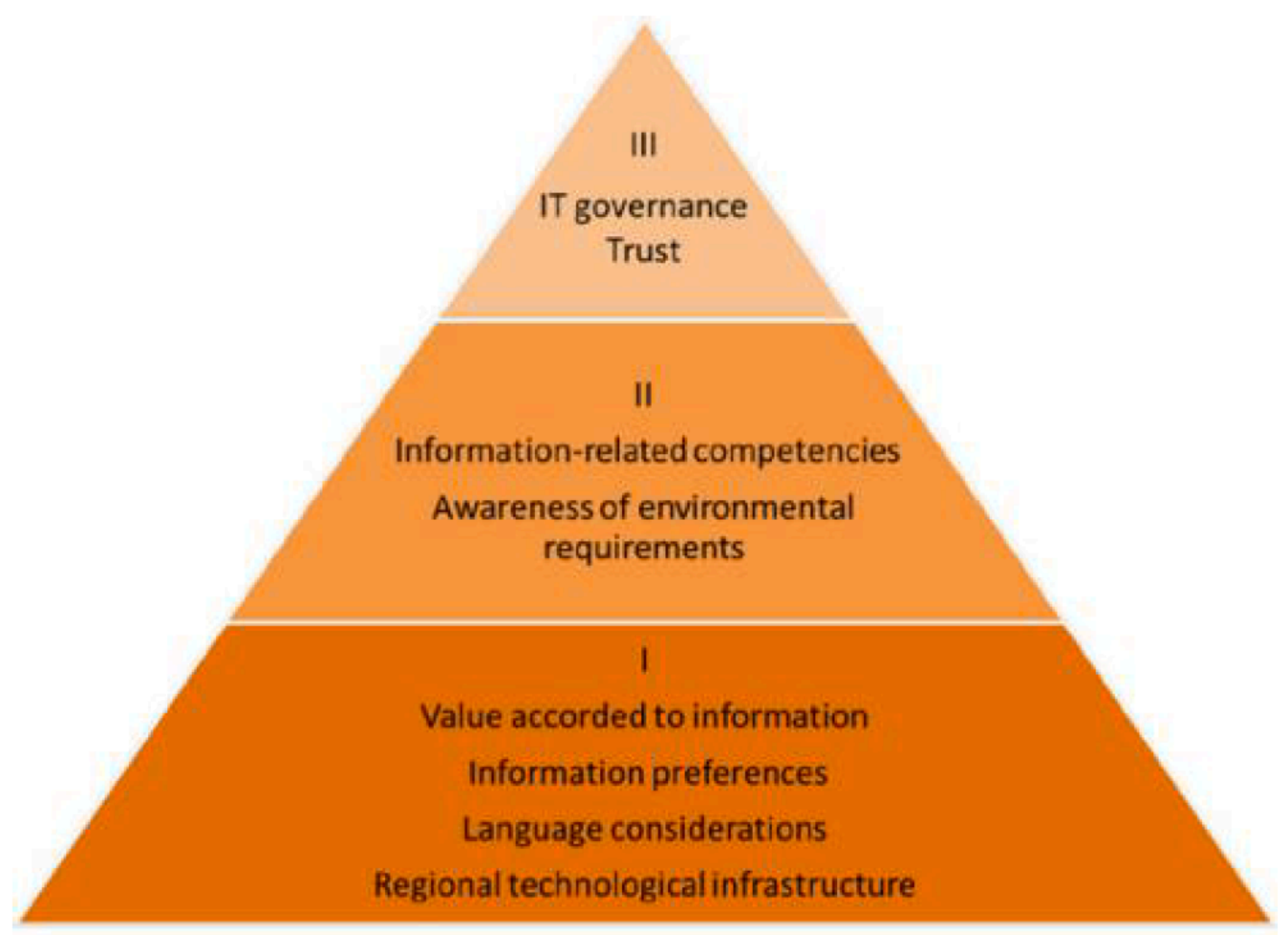

Figure 2.3: Information culture framework (Oliver \& Foscarini, 2014, p. 18; Oliver et al., 2018)

Svärd (2014) conducted case study research to identify the attitudes and norms that organisational employees in a medium-sized municipality in Belgium had towards the management of information and records, applying Oliver's (2011) information culture framework. This research confirmed that the attitudes that the different categories of employees had towards each other created barriers to promote information and management issues; reinforcing the organisational and cultural challenges faced by this particular organisation to encourage effective recordkeeping (Svärd, 2014, p. 17). 
More recently, Sundqvist and Svärd (2016) conducted an interpretative analysis of conceptualisations of information culture and its relation to information management and records management, through a comprehensive literature review. This analysis highlighted 'fuzziness' both in terms of definitions of information culture as well as the various ways that the concept of information culture is used within the studies: as an explanatory framework; as an analytical and evaluative tool; or as a normative standard (Sundqvist \& Svärd, 2016, p. 14). Sundqvist and Svärd (2016, p. 14) identified a lack of research examining information culture and records management, where just a few researchers have conducted empirical studies exploring the impact of cultural aspects on records management. In particular, Sundqvist and Svärd (2016, p. 14) argued that to date research has attempted to define information cultures with focus on information as an output of business processes, while the cultural impact on the input, how information is created, captured and preserved, has rarely been acknowledged; thus for information culture to function as an analytical framework, it needed to include how information is created, captured and preserved alongside how information is used, shared and disseminated. Ultimately, Sundqvist and Svärd (2016, p. 14) questioned whether there was a tangible correlation between information culture and recordkeeping behaviours.

\subsubsection{Governance and recordkeeping}

When examining how front-line public servants create, manage and use records, it is important to understand the context that this occurs within, the public governance framework, and thus impacting organisational culture and behaviours of individuals.

The two concepts of governance and recordkeeping are intrinsically linked. Harries (2009, p. 20) and Willis (2005, p. 90) reinforced this relationship by proposing that 
recordkeeping is one of the cornerstones of effective governance. Sound records management underpins, in a direct or indirect way, many of the vital aspects of governance at both a national and organisational level. As Dikopoulou and Mihiotis (2012, p. 133) stated, if the actions and decisions of an institution are derived from integrated, reliable and accurate records, governance cannot be questioned. Similarly, Kargbo (2010, p. 255) explained that the quality of governance in society is affected by the completeness and accuracy of records created by public servants when transacting public activities. Kargbo (2010) explored the relationship between governance and recordkeeping by focusing on development in Sierra Leone, viewing both governance and recordkeeping as pillars of development and as necessary concepts to promote democracy. "If good governance is to be attained, this must be underpinned by a solid policy framework that will set standards and guidelines for the creation, maintenance, corporation and coordination of records institutions" (Kargbo, 2010, p. 259).

Dikopoulou and Mihiotis (2012, pp. 137-138), Iacovino (2005, p. 255) and Willis (2005, pp. 86-87) outlined the central role that records play in governance, with legislation and public sector ethics performing important governance mechanisms. Analysis identified commonality between the specific ways that recordkeeping contributes to governance (Dikopoulou \& Mihiotis, 2012; Iacovino, 2005; Willis, 2005) (refer to Table 2.3).

Table 2.3: Key ways that recordkeeping contributes to governance

\begin{tabular}{|l|c|c|c|}
\hline & $\begin{array}{c}\text { Iacovino ((2005, pp. } \\
255-256)\end{array}$ & $\begin{array}{c}\text { Dikopoulou \& Mihiotis } \\
(2012, \text { pp. 137-138) }\end{array}$ & Willis (2005, p. 89) \\
\hline
\end{tabular}




\begin{tabular}{|c|c|c|c|}
\hline $\begin{array}{c}\text { Support } \\
\text { accountability }\end{array}$ & $\begin{array}{l}\text { Recordkeeping } \\
\text { contributes to personal, } \\
\text { organisational and } \\
\text { democratic } \\
\text { accountability, which } \\
\text { underpins an ethical } \\
\text { legal system. }\end{array}$ & $\begin{array}{l}\text { Accountability is } \\
\text { succeeded through } \\
\text { review of decisions and } \\
\text { transactions with } \\
\text { citizens and businesses, } \\
\text { internal or external } \\
\text { audits, parliamentary } \\
\text { monitoring, made } \\
\text { possible by records. }\end{array}$ & $\begin{array}{l}\text { Accountability - having } \\
\text { to answer for the things } \\
\text { one does. }\end{array}$ \\
\hline $\begin{array}{c}\text { Support } \\
\text { compliance } \\
\text { with } \\
\text { legislation }\end{array}$ & $\begin{array}{l}\text { Recordkeeping supports } \\
\text { legal and ethical rights } \\
\text { and obligations within a } \\
\text { socio-legal system. } \\
\text { Recordkeeping is } \\
\text { required to regulate } \\
\text { business and social } \\
\text { activity. } \\
\text { Recordkeeping forms an } \\
\text { integral part of the } \\
\text { governance of legal and } \\
\text { social relationships (for } \\
\text { example, doctor-patient, } \\
\text { citizen-state, parent- } \\
\text { child). }\end{array}$ & $\begin{array}{l}\text { Compliance with law } \\
\text { and statutory } \\
\text { framework can be } \\
\text { satisfied where } \\
\text { legislation requires } \\
\text { maintenance of records. }\end{array}$ & $\begin{array}{l}\text { Compliance - having } \\
\text { systems to ensure that } \\
\text { things are done } \\
\text { properly. } \\
\text { Meeting statutory and } \\
\text { common law } \\
\text { requirements - meeting } \\
\text { applicable legal } \\
\text { obligations. }\end{array}$ \\
\hline $\begin{array}{l}\text { Provide } \\
\text { evidence of } \\
\text { business } \\
\text { activities }\end{array}$ & $\begin{array}{l}\text { Recordkeeping provides } \\
\text { ongoing evidence or } \\
\text { proof of a particular } \\
\text { activity. }\end{array}$ & $\begin{array}{l}\text { Effective management } \\
\text { and appropriate access } \\
\text { to accurate and } \\
\text { complete public records } \\
\text { demonstrate processes } \\
\text { and activities have been } \\
\text { undertaken, executed, } \\
\text { reviewed, cancelled, etc. }\end{array}$ & $\begin{array}{l}\text { Due process - doing } \\
\text { things in an agreed, } \\
\text { documented, controlled } \\
\text { and appropriate way. }\end{array}$ \\
\hline $\begin{array}{c}\text { Ensure } \\
\text { transparency }\end{array}$ & & $\begin{array}{l}\text { Transparency is secured } \\
\text { since records held by an } \\
\text { agency make it possible } \\
\text { for people and bodies } \\
\text { who have a right or } \\
\text { obligation to be aware of } \\
\text { what has been done, } \\
\text { when and how has been } \\
\text { done, to get knowledge } \\
\text { of it. }\end{array}$ & $\begin{array}{l}\text { Transparency - doing } \\
\text { things in a way which is } \\
\text { open to appropriate } \\
\text { scrutiny }\end{array}$ \\
\hline
\end{tabular}




\begin{tabular}{|c|l|l|l|}
\hline $\begin{array}{c}\text { Protect secure } \\
\text { and restricted } \\
\text { records }\end{array}$ & $\begin{array}{l}\text { Policies and systems } \\
\text { used guarantee the } \\
\text { protection of secure and } \\
\text { restricted records. }\end{array}$ & $\begin{array}{l}\text { Security of personal and } \\
\text { corporate information - } \\
\text { having systems to } \\
\text { ensure protection of } \\
\text { information. }\end{array}$ \\
\hline
\end{tabular}

These five factors provide a basis for developing an understanding of the recordkeeping behaviours of front-line public servants within the context of both organisational and national governance. While the relationship between governance and recordkeeping is acknowledged, this relationship has not been explored in depth and the contribution of records management to good governance and accountability are often not recognised by other professions or organisational management (Isa, 2009, p. 2; Willis, 2005, p. 86).

In case study research to understand the relationship between records management and good governance, Isa (2009, pp. 248-251) identified two key factors that contributed to records management not being regarded as essential for good governance: a lack of awareness and commitment among senior management of the importance of good recordkeeping, and that records management was not embedded in businesses processes. Isa (2009, p. 256) also explored the relationship between recordkeeping, governance and risk, arguing that recordkeeping ensures the availability of records for risk assessment and systematically captures the records of risk management processes. However, Isa (2009, p. 257) found limited empirical evidence of this relationship within the case studies he conducted, with one exception; in one case study, the risk scorecards produced by the organisation's risk committees were subsequently used by the records management division to establish records retention schedules. The availability of reliable input meant the process of managing records was more economical as the priority was based on risks identified by the risk committees; consequently, records of higher risk were given 
higher priority in the records retention schedules than records of lower risks (Isa, 2009, p. 257).

\subsection{Chapter summary}

The subject of this study is extensive and multidisciplinary. This literature review has attempted to explore the main concepts, moving from the broadest concepts of recordkeeping and the nature of the public sector, linking them together with discussion on their connections to organisational behaviours, information culture and governance.

In order to provide context for this study, the literature review has explored the working environment of recordkeeping for front-line public servants. Salient aspects of this environment are that ICTs continue to significantly impact what public servants do, the ways they approach their roles and the ways that government representatives interact with citizens. As Schuppan (2015, p. 243) argued, the introduction of e-government and ICTs in public agencies changed the structure and dynamics of how work was organised; front-line public servants confront a wider variety of citizens' requests and they make decisions about the delivery of larger amounts of information from multiple information sources. Whilst this study does not conduct a comparative analysis between government processes and behaviours prior to the introduction of ICTs and in the digital age, assumptions are made that the working environment for public servants is significantly different from that prior to the introduction of ICTs. In addition to changing the working environment of public servants, this study also acknowledges that the introduction of ICTs has fundamentally changed the nature of recordkeeping.

Within this context, this study draws on three theories to form the analytical framework: recordkeeping informatics, personal information management, and the 
set of factors influencing the decision-making behaviours of front-line public servants.

Recordkeeping informatics is applied within this study as it recognises that recordkeeping behaviours of individuals occur within a broad and complex organisational context. While the approach is still emergent, it is underpinned by records continuum theory, which is a significant input to the conceptual thinking behind the setting of regulation and standards for recordkeeping within New Zealand. As well as being underpinned by records continuum theory, the approach recognises and provides a framework for analysis incorporating organisational culture, business processes and archival access to recorded information.

A challenge of drawing on personal information management strategies to understand recordkeeping behaviours is that personal information management strategies are solely focused on individual's behaviours, without appreciating the organisational and societal influences or legislative requirements for ensuring information is accessible beyond individual actions. Personal information management strategies do not acknowledge the existence of organisational records or the capture, organise or pluralise dimensions described within the records continuum. However, personal information management strategies provide valuable insights into individual recordkeeping behaviours, important in furthering the understanding of the recordkeeping behaviours of New Zealand front-line public servants in ICT-enabled environments. To address this limitation of personal information management strategies, the personal information management strategies are applied within this analytical framework that incorporates the recordkeeping informatics approach, with its broader focus on recordkeeping within business processes and organisational contexts. 
While the literature review explored research into factors that influence personal information management behaviours, this study has not leveraged these within the analytical framework for the reasons outlined whereby personal information management strategies are limited. In addition, the literature review explored the theoretical construct of information culture and, in particular, the information culture framework (Oliver, 2011; Oliver \& Foscarini, 2014; Oliver et al., 2018). Overall, while information culture is an interesting concept useful to explore the role of individual attitudes and the ways that organisations value information, this study does not use the information culture framework as a research lens. The information culture framework is structured according each element's susceptibility to change based on the organisation as the entity; which, while a meaningful approach for records managers and those responsible to guide recordkeeping within organisations, is limited in its ability to provide a taxonomy of factors that may influence the recordkeeping behaviours of public servants.

Instead, this study looked to public administration to understand factors that influence front-line public servants decision-making. Literature exploring the behaviours of public servants within government agencies is broad and multifaceted; within the context of this study, the focus of the literature reviewed is on studies that explore user behaviours and ICT-associated organisational change, with specific focus on studies exploring information-related systems, such as EDRMS (Anderson \& Robey, 2017; Lewellen, 2015; Strong et al., 2014). Influencing factors identified within these studies include the abilities and preferences of the individual, the features of the system and the wider organisational context in which the technologies are used (Anderson \& Robey, 2017, p. 111; Strong et al., 2014, p. 72). For the purposes of this study, these factors were found to be overly narrow in their focus; the four factors identified by Loyens and Maesschalck (2010, p. 72) presented 
an opportunity to explore a broad range of categories influencing the behaviours of public servants:

- Individual decision-maker characteristics, for example, professional norms, workers' beliefs, and moral values of front-line officers.

- Organisational characteristics, including the organisation's internal structure, workload pressure, rules and constraints, organisational culture, co-workers and supervisors.

- Client attributes, for example perceived level of need of the client.

- The broader community, including laws and regulations, the media and other service agencies.

Incorporating the four factors identified by Loyens and Maesschalck (2010, p. 72) in the analytical framework for this study, draws on public administration research, acknowledging that recordkeeping occurs within a specific working environment, in this study, the public sector, and in this way aligns with the organise and pluralise dimensions of the records continuum, a cornerstone of recordkeeping informatics.

In addition, the literature review identified five ways that recordkeeping contributes to governance through analysis of Dikopoulou and Mihiotis (2012), Iacovino (2005) and Willis (2005). While the concepts of governance and recordkeeping are intrinsically linked and this relationship between governance and recordkeeping is acknowledged, the implications of this relationship for public servants has not been explored in depth and the contribution of records management to good governance and accountability are often not recognised by other professions or organisational management (Isa, 2009, p. 2; Willis, 2005, p. 86). In the absence of existing empirical 
research, this framework provides a basis for understanding the governance implications of the recordkeeping behaviours of New Zealand front-line public servants. 


\section{Research design and methodology}

\subsection{Introduction to this chapter}

Creswell (2003, p. 5) suggested that there are three questions central to the design of any research:

- What claims of knowledge does the researcher make?

- What strategies of inquiry are informing the research?

- What methods of data collection and analysis does the researcher use?

This chapter describes the research design and methodology for this study, and in the process addresses each of these three questions.

\subsection{Claims of knowledge}

All research is based on underlying assumptions about what constitutes 'valid' research and what research methods are appropriate (Myers, 2009, p. 35). Also, all researchers bring to their research certain lenses for seeing the world and, therefore, make sense of it in different ways. To conduct research, identifying the research paradigm is a significant process, which consequently provides the study with a broad framework of philosophical perspectives. The research paradigm is underpinned by ontological assumptions about the nature of social reality and epistemological assumptions about how investigators obtain knowledge of this reality (Blaikie, 2007, pp. 12-13).

Ontology is the branch of philosophy that is concerned with the nature of what exists; in the social sciences, ontologies are used to answer the question "what is the nature of social reality?" (Blaikie, 2007, p. 13). Researchers may take two broad 
positions when referring to ontological positions: idealist and realist. An idealist position assumes that what is regarded as the external world has no independent existence apart from individual thoughts; in contrast, a realist position assumes that both natural and social phenomena have an existence that is independent of the activities of the human observer (Blaikie, 2007, pp. 12-13).

Closely related to questions of ontology are those concerned with epistemology. Epistemology refers to forms of knowledge, offering answers in the social sciences to the question "how can social reality be known?" (Blaikie, 2007, p. 18). There are two wide-ranging positions concerning an individual's epistemological beliefs: positivist and post-positivist. Positivism attempts to identify causal relationships and regularities between the constituent elements; this form of epistemology is based on the traditional approaches of the natural sciences. The second position, postpositivism, follows that the only way to understand the social world is by understanding the points of view of those under investigation.

This study applies an idealist ontological approach and post-positivist epistemology, and in this way, the philosophical paradigm for this research aligns with the interpretive tradition. Interpretive research seeks to "understand the deeper structure of a phenomenon which it is believed can then be used to inform other settings" and to obtain a shared understanding of the phenomenon (Orlikowski \& Baroudi, 1991, p. 5). This philosophical paradigm focuses on exploring meaning in context, with the aim to develop an in-depth understanding of the phenomenon under investigation (Myers, 2009, p. 39). The interpretive focus on meanings in action concentrates on participants' interpretations of their beliefs and practices (Rhodes, 2007, p. 1259), allowing participants to use their words and images, and to draw on their personal experiences (Orlikowski \& Baroudi, 1991, p. 15). Interpretive research involves researchers interpreting what they see, hear and understand; in 
this way, the researcher's interpretations cannot be separated from their backgrounds and prior understandings (Creswell, 2009, p. 176).

\subsection{Strategy of inquiry}

Strategies of inquiry provide the specific direction for procedures in the design of a study. This study consists of two phases that address the research objectives as outlined in Section 1.4; and that align with the interpretive paradigm with a specific focus on exploring meaning in context. The two research phases are as follows:

- Phase I: This study employed a case study approach as this strategy supports empirical inquiries investigating contemporary phenomenon, in this case recordkeeping behaviours, in depth and within their real-world context (Yin, 2014, p. 16). This phase addressed the first research objective: To develop an empirical understanding of recordkeeping behaviours of New Zealand frontline public servants within ICT-enabled environments.

- Phase II: This study employed focus groups, which explored the governance implications of the individual behaviours that were observed during the fieldwork, providing a macro-level perspective on the findings of the case studies. This phase addressed the second research objective: To explore the governance implications of the recordkeeping behaviours of New Zealand front-line public servants.

The two phases of the research design are discussed in more detail below.

\subsubsection{Case research}

The efforts of Bronislaw Malinowski, Frédéric Le Play and members of the Chicago School form the basis for the origins of case research (Hamel, Dufour, \& Fortin, 
1993). Polish-born Bronislaw Malinowski conducted a study on the Trobriand Islands, in Melanesia, for three years during World War I. He pioneered works of participant observation, where he catalogued every detail of the society under study, including the behaviours, beliefs and rituals that marked the life of the society. Malinowski's study of culture incorporated an understanding of the meanings that actors assigned to their patterns of behaviours, beliefs and rituals prevalent in their society (Hamel et al., 1993). The 19th-century works of Frederic Le Play also influenced early sociological case studies. Studying the working class populations of European nations, Le Play presumed that he could not study society as a single unit; as a result, he focused on the family as the social unit to provide a better understanding of the characteristic traits of society as a whole. The case study was the approach of choice for early sociological research in the United States at the end of the 19th and the beginning of the 20th centuries. These initial studies concerned small, local communities and urban neighbourhoods where rural and immigrant populations had recently settled. The University of Chicago soon became a leader of the case study approach in the United States. The case study approach, as applied by members of the Chicago School, was an experience containing the meanings and symbols involved in the interactions of the social actors. These meanings and symbols entered into the actors' interactions and defined their points of view on these interactions (Hamel et al., 1993).

Yin (2014, p. 16) defined case research as "an empirical inquiry that investigates a contemporary phenomenon (the 'case') in depth and within its real-world context, especially when the boundaries between phenomenon and context may not be clearly evident". Benbasat, Goldstein and Mead (1987, p. 371) outlined a series of the characteristics of case research (refer to Table 3.1).

Table 3.1: Case research strategy characteristics 
(Benbasat et al., 1987, p. 371)

\begin{tabular}{|l|}
\multicolumn{1}{|c|}{ Characteristics of case research strategy } \\
\hline The phenomenon is examined in a natural setting. \\
\hline Data are collected by multiple means. \\
\hline One or few units are examined. \\
\hline The complexity of the unit is studied intensively. \\
\hline $\begin{array}{l}\text { Case studies are more suitable for the exploration, classification and hypothesis development } \\
\text { stages of the knowledge building process; the investigator should have an open attitude } \\
\text { towards exploration. }\end{array}$ \\
\hline No experimental controls or manipulation are involved. \\
\hline The investigator may not specify the set of independent and dependent variables in advance. \\
\hline The results derived depend heavily on the integrative powers of the investigator. \\
\hline $\begin{array}{l}\text { Changes in site selection and data collection methods could take place as the investigator } \\
\text { develops new hypotheses. }\end{array}$ \\
\hline $\begin{array}{l}\text { Case research is useful in the study of "why" and "how" questions because these with } \\
\text { operational links to be traced over time rather than with frequency or incidence. }\end{array}$ \\
\hline The focus is on a contemporary phenomenon. \\
\hline
\end{tabular}

These characteristics align with the overall purpose and research questions for this study: an explorative study of a contemporary phenomenon in a natural setting, where no experimental controls or manipulations are involved.

While the characteristics of case research align strongly with the overall aims of this research, there are also weaknesses to this research strategy that must be acknowledged and considered. Cavaye (1996, p. 229) outlined weaknesses of case research, including that it is not possible to generalise findings statistically to a population, the researcher does not have control over independent variables, which limits the internal validity of any conclusions, and that case research cannot necessarily indicate the direction of causation between variables. 
As outlined by Yin (2014, p. 21), case studies are generalisable to theoretical propositions and not to populations; the goal of case research is to expand and generalise theories and not to extrapolate probabilities. Walsham (1995, p. 79) outlined four types of generalisations that are possible from interpretive case research: the development of concepts, the generation of theory, the drawing of specific implications, and the contribution of rich insight. Thus, as Walsham (1995, p. 79) described, researchers should view findings from case research as 'tendencies' that are valuable to explain other situations but are not wholly predictive for future scenarios.

Cavaye (1996, p. 229) also stated that there are limits to the internal validity of any case research conclusions and that case research cannot indicate the direction of causation. Yin (2014, p. 19) also suggested that possibly the greatest concern in doing case study research related to a presumed need for greater rigour. Creswell (2014, p. 201) described validity as referring to the researcher checks for the accuracy of the findings by employing certain procedures. For this study, triangulation and member checking are two validity strategies employed to address the need for rigour (refer to Section 3.4.3).

There are similarities between case research and other research strategies, including ethnography. While this research did not follow an ethnographic strategy of inquiry, ethnographically-informed approaches influenced this study, including previous ethnographic research conducted in the recordkeeping discipline (Gracy, 2004; Meehan, 1986; Shankar, 2004; Trace, 2002; Yakel, 1997, 2001) and the public administration discipline (Bjerregaard \& Klitmoller, 2010; Boll, 2015; Buffat, 2015; Noordegraaf \& Stewart, 2000; Pors, 2015; Rhodes, 2007). Ethnography involves the researcher participating, either overtly or covertly, in people's daily lives for an extended period of time, gathering data from a range of sources, including watching 
what happens, listening to what is said, collecting documents and artifacts as well as asking questions through interviews (Hammersley \& Atkinson, 2007, p. 3). The distinguishing features of ethnography include a longitudinal approach (for example, a presence in the field of at least a year) and the use of participant observation (Cavaye, 1996, p. 230), neither of which were possible with the agencies involved in this research or within the time constraints of this doctoral study. However, the use of ethnographically-informed approaches within this study supported the exploration of the context for recordkeeping behaviours, including the influence of individual characteristics, client attributes, organisational culture, which encompasses features that reflect the geographical situation of the organisation, the occupations of people who work within it, and any cultural characteristics that are unique to that organisation (Oliver, 2011, pp. 22-23), as well as the broader community.

\subsubsection{Multiple case research}

Where more than one cases are studied jointly to investigate a phenomenon, population, or general condition, Stake (2005, p. 445) referred to this as a multiple case study, a variant of the single case research strategy. Conducting multiple case studies better supports generalising the research findings and helps to strengthen the precision, validity, and stability of the research (Benbasat et al., 1987; Miles \& Huberman, 1994; Yin, 2014). As Benbasat et al. (1987, p. 373) explained, multiple case studies provide opportunities for cross-case analysis and are useful when the intent of the research is to build a rich description, theory building or theory testing.

Three case studies were conducted within this research to support the development of a broad knowledge basis, acknowledging the lack of generalisability arising from conducting single case research. The findings derived from the multiple case studies enable the comparison of evidence across cases and support better data 
triangulation. Completion of the three case studies was also pragmatic within the confines of this doctoral study.

The selection of sites was limited to state sector agencies where staff worked directly with the New Zealand public. The agencies involved included Inland Revenue Department and Te Puni Kōkiri, the Ministry of Māori Development (two public service departments) and Environmental Risk Management Authority (a statutory Crown entity). Agreement from all three agencies was provided by senior management and outlined that while the organisation would be identified, information relating to individuals involved in the fieldwork would be kept confidential and reported only in a non-attributable form.

The three sites reflect diversity across a range of attributes (refer to Table 3.2).

Table 3.2: Attributes of the case study sites

\begin{tabular}{|c|l|l|l|}
\hline Agency size & ERMA New Zealand & \multicolumn{1}{|c|}{ Te Puni Kōkiri } & \multicolumn{1}{|c|}{ Inland Revenue } \\
\hline $\begin{array}{c}\text { Small: 90 full-time } \\
\text { (Envivalent employees } \\
\text { Management } \\
\text { Authority, 2010, p. 34) }\end{array}$ & $\begin{array}{l}\text { Medium: 261 full-time } \\
\text { equivalent employees } \\
\text { (State Services } \\
\text { Commission, 2015, p. } \\
51 \text {. }\end{array}$ & $\begin{array}{l}\text { Large: 5,681 full-time } \\
\text { equivalent employees } \\
\text { (State Services } \\
\text { Commission, 2015, p. } \\
51 \text { ) }\end{array}$ \\
\hline $\begin{array}{c}\text { Availability of } \\
\text { EDRMS }\end{array}$ & EDRMS & EDRMS & Shared drive \\
\hline $\begin{array}{c}\text { Location of the case } \\
\text { study site }\end{array}$ & Head office & Regional office & Regional office \\
\hline $\begin{array}{c}\text { Relationship with } \\
\text { citizens }\end{array}$ & $\begin{array}{l}\text { Limited to citizens } \\
\text { seeking to introduce } \\
\text { new organisms to } \\
\text { New Zealand }\end{array}$ & Predominately Māori & $\begin{array}{l}\text { Potentially all New } \\
\text { Zealand citizens }\end{array}$ \\
\hline $\begin{array}{c}\text { Primary } \\
\text { communication } \\
\text { methods with } \\
\text { citizens }\end{array}$ & Telephone and email & $\begin{array}{l}\text { Telephone, email and } \\
\text { face-to-face }\end{array}$ & Telephone \\
\hline
\end{tabular}




\subsubsection{Researcher's position}

Certain aspects of the strategy of the inquiry were influenced by the researcher's previous experiences, prior knowledge and personal situation.

As a practitioner I have been involved in the recordkeeping community within New Zealand for a number of years, which provided some opportunities involving the fieldwork that might have otherwise been unavailable. In particular, I was employed at ERMA prior to initiating contact regarding involvement in this research; therefore, the senior managers who received the initial letter of engagement would have been familiar with my name and my professional interests and likewise, once ERMA agreed to be a case study site, the individual participants were also familiar with me and my professional interests. I had no prior knowledge or relationship with the other two case study sites involved in this research.

My involvement in the recordkeeping community did not, however, reduce the challenges associated with identifying and securing cases. There was only a small number of agencies interested in participating from which to select the case sites, and once case sites were confirmed, only a small number of participants agreed to be involved.

There was also a gap between the two stages of the research amounting to a number of years, owing to maternity leave that I took after the birth of my children.

\subsection{Methods of data collection and analysis}

The design for this inductive study consisted of two data collection phases. The study began with multiple case studies that sought to explore the recordkeeping behaviours of front-line public servants. The second data collection phase involved a series of focus groups that explored the wider implications of the observed 
recordkeeping behaviours, with a specific focus on understanding if the findings from the case studies were still relevant given the amount of time that had passed since conducting the case studies and on exploring governance implications of individual recordkeeping behaviours.

\subsubsection{Multiple case studies}

The purpose of the first data collection phase of the research, involving nonparticipant observations and semi-structured interviews, was to explore the recordkeeping behaviours of front-line public servants in situ. Documentation was also used as a source of evidence within this data collection phase.

Agencies that met the predefined selection criteria were invited to be involved; and for the small group of agencies that were interested in being involved, the researcher obtained formal approval from a senior manager or the Chief Executive before initiating any empirical research. The researcher also obtained approval to conduct the non-participant observations and semi-structured interviews for the case study phase from the Pipitea Human Ethics Committee at the Victoria University of Wellington.

Once the agency approved its involvement and was ready to proceed with the fieldwork, the researcher requested volunteers. Between three and five volunteers from the same team unit were invited to be involved where they would participate in one semi-structured interview each and a series of three observations in their normal working environment. In this way, there was already some degree of bias based on the voluntary involvement of participants as they were not randomly selected to be involved, which suggests they may have already been interested in recordkeeping to some degree. The researcher also requested semi-structured 
interviews with recordkeeping and ICTs managers at the three organisations (refer to Appendix A for a list of the 22 participants involved in this phase of the study).

Before the first observation, the researcher conducted interviews with each participant, involving a predetermined set of topics (refer to Appendix B for the set of topics addressed during these interviews). The semi-structured nature of the interview topics allowed for questions to be pre-formulated but set no requirement for strict adherence to them (Myers, 2009, p. 124). The questions were formed drawing on concepts discussed within the literature review. Participants' understandings of the legislative framework were also explored. The interview questions did not include a definition of records; the researcher risked biasing the interviews by stating definitions that might have been in conflict with the participant's prior understanding of the term.

Non-participant observations were conducted with each participant to develop an understanding of the recordkeeping behaviours of each participant. Tope, Chamberlain, Crowley and Hodson (2005, pp. 473-474) defined non-participant observation as observation in the field where the researcher is present while the activity takes place, observing what transpires but is not participating. This form of observation was conducted as the observations allowed data to be collected in a natural setting, supporting the capture of activities as they happened and any unusual aspects to be noted without disrupting participant's work (Creswell, 2009, p. 179). During each observation, field notes were taken, collecting both descriptive notes (including descriptions of physical locations, ICTs, and interactions with colleagues and citizens) and reflective notes. However, obtaining empirical data using non-participant observations is dependent on the researcher having good observation skills and ensuring that the participant does not feel intruded upon or that the research is overly intrusive (Creswell, 2009, p. 179). Researchers must be 
aware that individuals being observed may alter their behaviour in the scholar's presence (Gillespie \& Michelson, 2011, p. 262).To mitigate these concerns, and to minimise the researcher's impact on the research environment, the participants defined the time and place of the observations and during the observations engagement between the researcher and the participants was kept to an absolute minimum. Each participant was observed on three occasions; for some participants, they were not available on those particular days, in which case not all of the planned observations were conducted. Establishing trust with the participants and with the case study organisations was critical to facilitating the research relationship (Gillespie \& Michelson, 2011, p. 262). Combining the initial observations with the interviews was designed with the intention of increasing trust (J. Cooper, Lewis, \& Urquhart, 2004). Likewise, offering to share and discuss fieldwork with the organisations at the conclusion of the fieldwork, independent of the case study chapter, was one way of offering a tangible benefit to the three case organisations. Conducing longer observation periods over longer time period may also have increased trust between the researcher and research participants and reduced any feelings of intrusion; however, constraints on the study meant that longer periods were not possible. Engagement between the participants and the researcher varied between the three case studies. Some participants were more relaxed, engaging with the researcher in an informal and casual manner, as well as actively participating in the interview; while other participants were somewhat nervous, cautious and formal in their responses. The varied engagement is likely to be a combination of personal characteristics and organisational contexts impacting on the level of engagement with the researcher. The researcher employed various approaches to respond to this, including discussing Chief Executive level approval to conduct the research and the validation process to be followed with their responses and the case study.

Emphasising this study's and the researcher's independence from regulatory bodies, 
both internal and external to the organisation, also minimised risks of biasing the interviews towards responses based on how participants expected they should respond (Viseu, Clement, \& Aspinall, 2004, p. 95). The interviews were conducted either at the participants' desks or in a quiet room nearby to ensure the participant had access to any information they might require to answer the questions and to reduce feelings of discomfort.

Documentation was also a source of evidence within this data collection phase. As outlined by Yin (2009, p. 106), documentary sources can be advantageous as they are stable, unchanging and able to be repeatedly accessed; they are also unobtrusive as they are not created for or in response to the case study. However, documentary sources potentially involve selective bias if the collection of documentation is incomplete, reporting bias as the documents reflect the unknown bias of the authors, as well as challenges relating to retrievability and access. Documentary sources used in this study included annual reports, website content, reports available made public by the agencies, as well as internal documentation provided voluntarily by the agencies, including the ERMA New Zealand Code of Conduct and Records Management policy as well as training material on the use of metadata by Te Puni Kōkiri.

The process of data analysis for this data collection phase included preparing the data for analysis, conducting different analyses, developing codes and themes to make representations of the data and making an interpretation of the larger meaning of the data (Creswell, 2009, p. 190). NVivo, the qualitative data management software, was used during this analysis stage. Field notes from the observations were prepared for analysis, and the audio-recorded interviews were transcribed. Once transcribed, the outputs of the interviews and the observations were coded, seeking to identify a cohesive set of themes. The codes emerged during the data 
analysis and were refined based on concepts from the literature review. Once an initial code of the data for each case study was completed, the researcher refined the codes and identified relationships between the codes. This process was an iterative task, as when the data collection for each case study was conducted, the set of codes was adjusted and refined (see Appendix C for the final set of codes).

This study is informed by the analytical framework, which encompasses the three theoretical concepts explored within Chapter 2: recordkeeping informatics, personal information management theories, and the influencing factors on front-line public servants' decision-making behaviours. All three frameworks influenced the codes used for data analysis. Recordkeeping informatics is used in the discussion and analysis (Chapter 8 ) to understand how participants engaged with records across the three facets for analysis. The personal information management strategies of piling, filing and structuring provide meaningful ways of describing individual's information management behaviours and are used as a means to identify specific recordkeeping behaviours from the case studies and the focus groups. The analysis of the fieldwork also applies the four influencing factors on front-line public servants' decision-making behaviours to explore their applicability to the observed recordkeeping behaviours. The frameworks are explored in Chapter 2 and are applied to the analysis in Chapter 8.

\subsubsection{Qualitative focus groups}

The second data collection phase of the study involved conducting a series of three focus groups to build an understanding of the wider implications of the recordkeeping behaviours identified during the case study fieldwork. The focus groups were designed to explore macro-level perceptions associated with the individual recordkeeping behaviours identified during the case study fieldwork. 
The focus group is a qualitative data gathering technique whereby several people are systematically questioned simultaneously (Fontana \& Frey, 2005, p. 703). Group interviews have some advantages over individual interviews, as they are relatively inexpensive to conduct, often producing rich data that are cumulative and elaborative, they can be stimulating for respondents aiding in recall and interactions, and the format is flexible (Fontana \& Frey, 2005, pp. 704-705). Focus groups were used at this stage of the study as they provided an opportunity to collect multiple understandings and meanings within a group environment and to explore the degree of consensus on the topic. This opportunity for interaction among participants was a valuable one. The focus groups also provided a chance to test and elaborate on the relevance of the case study findings with different audiences.

The researcher obtained approval to conduct the focus group meetings for the second phase of data collection from the Pipitea Human Ethics Committee at the Victoria University of Wellington. Three focus group meetings were held between July and August 2016. Each focus group involved between seven and eight participants (refer to Appendix D for the list of the focus group participants). Focus group participants were purposefully invited, with a specific focus on arranging one focus group for interested recordkeeping professionals from a broad range of organisations (focus group meeting one), one meeting with representatives from recordkeeping and information management authorities (focus group meeting two) and the third with records managers (focus group meeting three).

Invitations to the first focus group meeting were sent to the New Zealand recordkeeping email distribution list, inviting all members. Eight participants with varying recordkeeping roles attended the meeting, held at a venue in central Wellington, convenient to public servants. The opening of the meeting was used to introduce both the researcher and the research conducted to date, and the majority 
of this first focus group meeting was spent discussing the three case studies. Each case study was summarised, providing the broad context of the case study, and one notable finding from each case was discussed, to understand if the findings from the case studies were still relevant given the amount of time that had passed since conducting the case studies and the range of organisations represented at the meeting. The discussion also explored whether the participants could relate to the findings (refer to Appendix E for the discussion topics discussed at Focus Group 1).

Participants for the second and third focus groups were purposely selected: for the second focus group meeting specific organisations were asked to nominate volunteers (refer to Appendix F for a list of the organisations contacted). Records managers at state sector agencies were invited to the third focus group meeting. The structure for the meetings was consistent for these two focus group meetings. The opening of the meeting was used to introduce the researcher and the research; then participants discussed three discussion topics prepared by the researcher through analysis of the case studies (see Appendix G for the slide deck used during these two focus group meetings). The researcher introduced each discussion topic by providing some context derived from the case studies and asked open-ended questions. At the conclusion of the allocated one-hour time scheduled for all three of the focus group meetings, the researcher summarised the discussions and thanked the participants for their involvement.

During the focus group meetings, the role of the researcher was to direct the inquiry and interaction among the participants. Potential issues with focus groups include that one or a small coalition of persons can dominate the group, that obstinate respondents do not participate, or that responses are not obtained from the entire group, and thus the fullest coverage of the topic is not obtained (Fontana \& Frey, 2005, p. 704). The researcher moderated the discussion as required to mitigate these 
potential issues, although in all three meetings the conversation flowed evenly amongst members, kept to the topic, opportunities for opposing opinions were encouraged, and participants were engaged in the discussions. The focus group meetings were audio recorded to allow the researcher to fully concentrate on the discussion without being required to take notes.

Data analysis of the focus group data followed a similar process to analysing the data gathered during the case study phase of this study. As the initial code identification for the three case studies was conducted before the facilitation of the focus groups, a set of codes was available. However, the codes were expanded to incorporate codes specifically discussed during the focus group meetings. Once all the data from both data collection phases was prepared and initial coding completed, the full set of codes was examined and refined. The coding of all data was reviewed to ensure adequate descriptions of the codes were used and that the coding was consistent across all data (refer to Appendix $C$ for the final set of codes).

The analysis of the focus groups was conducted alongside the case study data, allowing for rich description of both the micro-level recordkeeping behaviours and the macro-level perceptions associated with the individual recordkeeping behaviours. This comparative analysis involved the application of recordkeeping informatics, personal information management theories, and the influencing factors on front-line public servants' decision-making behaviours (Chapter 8).

\subsubsection{Tactics for confirming findings}

Qualitative analyses can be evocative, illuminating, masterful, but they can also be wrong (Miles \& Huberman, 1994, p. 262). This study adopted two key strategies to check the accuracy of the findings: triangulation and 'member-checking'. 
Triangulation involves using different data sources of information:

in effect triangulation is a way to get to the finding in the first place - by seeing or hearing multiple instances of it from different sources by using various methods and by squaring the finding with others it needs to be squared with (Miles \& Huberman, 1994, p. 267).

Combining various methods to triangulate data allows researchers to use different methods in different combinations; "the more methods used to study them [people], the better our chances will be to gain some understanding of how they construct their lives and the stories they tell us about them" (Fontana \& Frey, 2005, p. 722). The case study phase of this study involved multiple sources of evidence, including nonparticipant observations, semi-structured interviews with multiple participants from the same team, field notes collected by the researcher, documentation as well as interviews with managers at the organisations. Combining observations and interviews, along with these additional sources of evidence, provided the ability to triangulate during the case study phase of the study. Conducting multiple cases also gave an opportunity for data triangulation. The focus groups provided a further form of triangulation, as it provided a different source and different method of data collection, discussing the findings from the fieldwork.

Member-checking was also used to determine the accuracy of the qualitative findings by taking the transcripts and the findings back to the participants and determining whether these participants felt that they were accurate (Creswell, 2003, p. 196). During this study, member-checking was conducted on two separate occasions. The case study participants were provided with drafts of the cases for feedback and comments, and presentations were made to interested representatives from the three organisations, summarising the preliminary case study findings. The 
focus groups also provided a critical opportunity to review and discuss themes derived from the case studies.

\subsubsection{Ethical considerations}

Researchers conducting inductive studies enter the field with the goal of observing natural behaviour while taking steps to ensure they do not influence activity; from an ethical perspective, anonymity is impossible, while confidentiality is essential (Librett \& Perrone, 2010, p. 729). With research involving human participants, ethical concerns revolve around topics of:

- Informed consent: has consent been received from the participant after carefully and truthfully informed them about the research?

- The right to privacy: is the identity of the participant protected?

- Protection from harm: is the participant protected from physical, emotion or any other kind of harm? (Fontana \& Frey, 2005, p. 715).

To ensure the participants were fully informed about the purpose and use of the information they provided, each participant received an information sheet before their involvement in the interviews, observations or the focus group meetings. Each participant signed a consent form allowing data they provided to be used in the study. The information sheet, consent form and all interview and focus group topics and questions were approved by the Pipitea Human Ethics Committee at the Victoria University of Wellington before initiating the data collection phases of the study.

All opinions and vignettes have been reported using pseudonyms, protecting the participants' identities. All material, both physical and digital, is kept in protected 
locations (locked filing cabinet and password-protected digital files) and access is restricted to the researcher and the supervisors. Also, the researcher will retain field notes and notes from the focus group meetings as well as all audio and video recordings for five years after the conclusions of the study in case questions come up in an examination, or later that can only be resolved by re-examining the data. The regional offices of Te Puni Kōkiri and Inland Revenue involved in this research have also not been identified to protect the participants' identities.

In the course of this study, participants were not put in harm's way, physically, emotionally or in any other way. However, to minimise any concerns participants might have had, the researcher ensured that the focus group participants understood that any opinions they provided would be reported in aggregated form in such a way that individuals or the agencies where focus group participants worked are not identifiable.

\subsection{Chapter summary}

In summary, this chapter discusses the ontological premises that inform the research process and the epistemological basis for the methodological choices made in conducting this study. A qualitative case study research strategy is applied during the data gathering process to collect information on the recordkeeping behaviours of New Zealand front-line public servants. Conducting this case study in the interpretive paradigm aligns with the nature of the research question.

Case research fieldwork collected data to develop the three case studies that observed the recordkeeping behaviours of individuals within New Zealand public agencies and a series of focus group meetings were conducted. The combination of these research methods provides a rich set of information from which to derive an understanding of both the recordkeeping behaviours of New Zealand front-line 
public servants in ICT-enabled environments and the governance implications of these recordkeeping behaviours of New Zealand front-line public servants.

The following three chapters of this dissertation present the three case studies in the order that they were conducted, using vignettes to describe specific situations observed during the fieldwork. Chapter 7 presents the findings from the focus groups. 


\section{Recordkeeping behaviours at Environmental Risk Management Authority}

This chapter describes the first of the three case studies, conducted with the New Organisms unit of the Environmental Risk Management Authority (ERMA New Zealand) between November and December 2010. This chapter introduces the Wellington-based agency, including its role, its main functions as well as the organisational makeup, with a specific focus on outlining the activities of the New Organisms unit. The case study then describes the recordkeeping behaviours demonstrated by the participants.

\subsection{The Environmental Risk Management Authority}

In 2010, at the time of the fieldwork, ERMA New Zealand was a crown entity, established under the Hazardous Substances and New Organisms (HSNO) Act 1996. The agency was responsible for the protection of the environment and the health and safety of people and communities in New Zealand through the prevention or management of adverse effects of hazardous substances and new organisms. ERMA New Zealand made decisions that controlled the transhipment, importation, manufacture, use, storage and disposal of hazardous substances and new organisms in New Zealand. The subject of this case study is ERMA New Zealand; however, the agency was disestablished on 30 June 2011, and at that date, the functions were incorporated into a newly established agency, the Environmental Protection Agency (EPA).

ERMA New Zealand had two main functions; the first was to consider applications for the approval of hazardous substances and new organisms. ERMA New Zealand applied specific conditions to manage any risks associated with approved hazardous substances and new organisms; if the risks could not be managed appropriately, the application for the importation of a new substance or organism was declined or 
removed (Environmental Risk Management Authority, 2010, p. 13). The second main function of ERMA New Zealand was to monitor and review the enforcement of the HSNO Act, which involved conducting inquiries into incidents involving hazardous substances and new organisms, as well as monitoring and reviewing the extent that the HSNO Act reduced adverse effects on people and the environment. ERMA New Zealand monitored the impact of these interventions by analysing the number and severity of incidents (Environmental Risk Management Authority, 2010, p. 13).

ERMA New Zealand carried out these two main functions under the leadership of the Chief Executive. Staff were organised into five units: Hazardous Substances, New Organisms, Kaupapa Kura Taiao (the Māori unit), Strategy and Analysis, and Corporate Services. A General Manager managed each of these units, who reported to the Chief Executive. At the time of the fieldwork, ERMA New Zealand employed 90 full-time equivalent staff located at a single Wellington office (Environmental Risk Management Authority, 2010, p. 34).

\subsubsection{Information management at ERMA New Zealand}

As public servants, ERMA New Zealand staff worked within an environment with specific responsibilities based on New Zealand's legislative framework for recordkeeping (described in Section 2.5.1). The responsibilities derived from this framework were set out within the agency's Code of Conduct, the records management policy, information management procedures, as well as procedures and guidelines for handling confidential information. ERMA New Zealand published these documents on the Intranet, and new staff received training on recordkeeping responsibilities as part of the induction process.

The ERMA New Zealand Code of Conduct, which all staff were required to agree to and sign at the start of their employment, set out the minimum standards of conduct 
for employees. It outlined expectations for staff relating to personal responsibilities, external relationships, customer service, conflict of interests, upholding the reputation of the agency, as well as the expectations for handling confidential information (Environmental Risk Management Authority, 2008).

The requirements set out by the Code of Conduct were further extended by the records management policy (Environmental Risk Management Authority, 2009). The purpose of this policy was to provide a framework and to assign responsibilities for ensuring that full and accurate records of ERMA New Zealand's business activities were created, maintained, accessed and disposed of in a controlled manner (Environmental Risk Management Authority, 2009). A participant described the records management policy at ERMA New Zealand as:

ERMA's policy reflects the laws, the government policy. You should keep all copies of important emails. In fact, you should be writing notes from telephone conversations because it is retrievable under the OIA [...] You're aware that you need to keep documents should they need to be retrieved.

(Sharon, ERMA New Zealand)

The HSNO Act stipulated requirements to ensure full and informed public participation in the approval process; however, there was a potential conflict between this objective and the commercial sensitivity of specific records that applicants may include as part of an application. The principles for handling confidential information provided as part of applications were set out within a section of the ERMA New Zealand Code of Conduct as well as in a policy concerning confidential information handling. These documents included requirements for advisors to enter confidentiality agreements, for confidential information to be stored in a separate locked cabinet and for limits to the inclusion of 
confidential information in any evaluation or review reports (Environmental Risk Management Authority, 2008).

\subsubsection{Information technology at ERMA New Zealand}

Since ERMA New Zealand's establishment, a series of different document and records management systems (both physical and digital) had been implemented, each of which had left a unique legacy. One of the participants described this situation:

\footnotetext{
Glenn: "I' $m$ in my third filing system since I've been here. And I think I, well we got training for each one, probably more training for this last round because it's been electronic and it's been a bit more complicated..." Interviewer: "How long have you worked at ERMA New Zealand?" Glenn: "Six years." Interviewer: "And, there have been three filing systems in six years?" Glenn: "Yep, the F drive, the $\mathrm{R}$ drive and the EDRMS. And plus having to learn the paper system, and now being almost totally electronic, apart from paper originals, which we still file hard copies of."
}

ERMA New Zealand implemented the electronic document and records management system (EDRMS) between 2009 and 2010 using the Microsoft SharePoint product suite, which integrated with other Microsoft products used by the agency. 


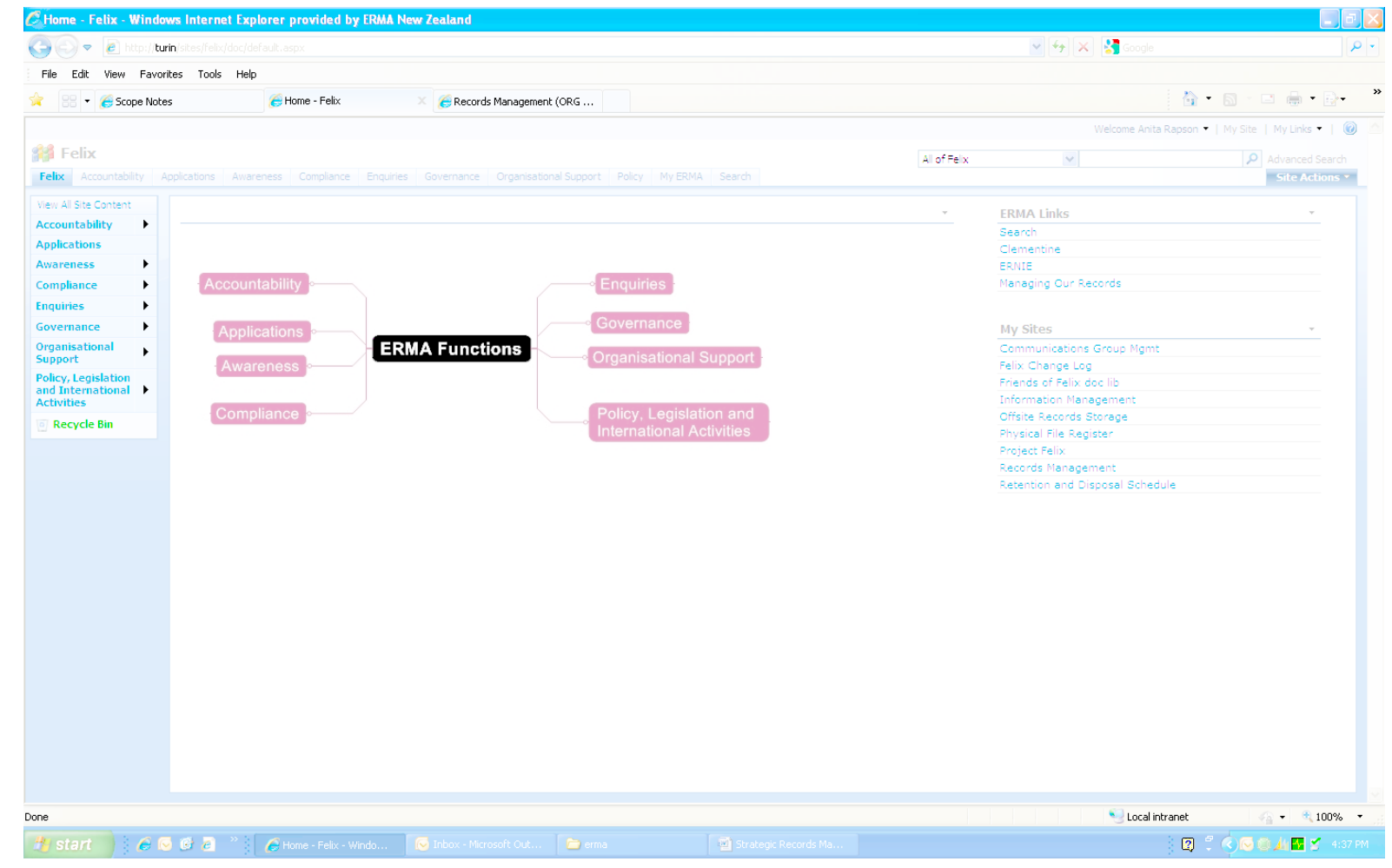

Figure 4.1: EDRMS homepage

The EDRMS was structured according to a functional classification structure, reflecting the functions and activities of the agency (refer to Figure 4.1 for a screenshot of the EDRMS homepage). Metadata was used to refine the classification relevant for each document further. Staff saved digital documents into one of the nodes of this classification, each of which corresponded to a SharePoint document library, and completed the metadata properties for each item. Depending on the area of the classification the item was created in, some metadata properties were prepopulated (refer to Figure 4.2 for a screenshot of how the metadata properties for a new blank document appeared when working in Microsoft Word). 


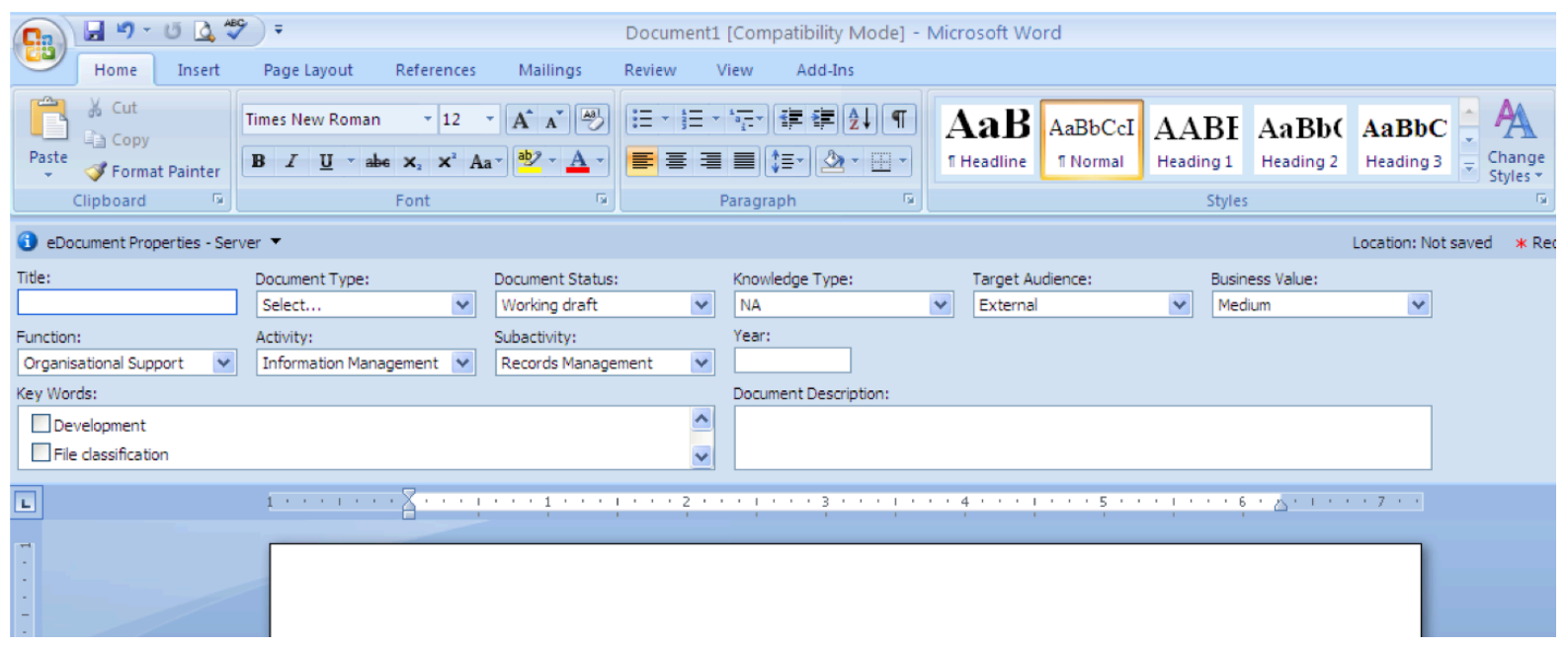

Figure 4.2: New document metadata profile, including default metadata

Staff used another system, Colligo, to save emails from Microsoft Outlook to the EDRMS. Using Colligo, staff were able to drag emails from Outlook and drop them into the EDRMS. During this process, Colligo automatically extracted some metadata from the email and populated relevant properties in the metadata profile for the email (refer to Figure 4.3 for a screenshot of the email metadata profile window that appeared when staff used Colligo to drag emails to the EDRMS).

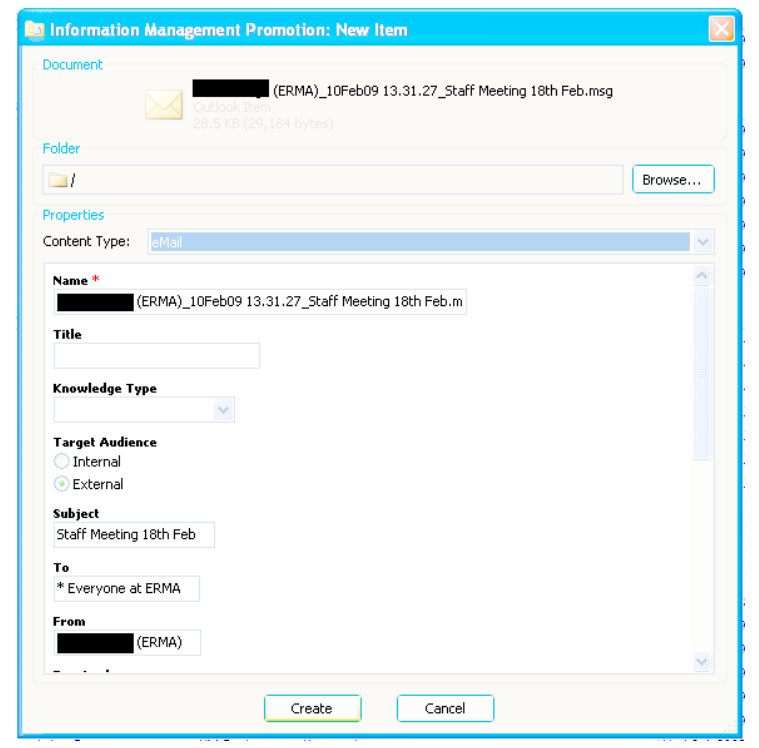

Figure 4.3: Example of email metadata profile in the EDRMS 
The EDRMS also integrated with the customer relationship management system (CRM), which was implemented at a similar time as the EDRMS. The CRM was designed to capture details about interactions between ERMA New Zealand staff and external parties. Staff lodged all enquiries and interactions with applicants in the CRM. The CRM allowed ERMA New Zealand to capture details on all interactions that individuals had with the agency, to follow a trail of interactions during an application process, to maintain contact details of the people who interact with ERMA New Zealand and to identify trends across enquiries and applications.

At the time the fieldwork was conducted, both the EDRMS and the CRM had been used by staff across the whole agency for just over six months; parts of the agency, including New Organisms, had been using the two systems for nearer to a year as they were involved in the project pilot implementation. These changes meant that while staff were using both of the systems and had received training, the systems and corresponding ways of working were still in the process of being fully incorporated into the agency's business processes.

\subsubsection{The New Organisms unit}

This case study is specifically focused on the New Organisms unit within ERMA New Zealand. The role of the New Organisms unit was to manage potential risks from organisms new to New Zealand. The unit considered applications for the approval of new organisms to New Zealand, including applications to import and release plants and animals and to conduct research in a laboratory.

Staff used different types of documents as part of the consideration of applications for new organisms, including documented interactions between ERMA New Zealand staff, applicants and submitters, research on the new organism mostly sourced from online scientific journal articles, as well as evaluation and decision 
reports. The decision-making could be queried at any time during the application and decision-making process or many years after the decision was made, and therefore throughout the application process, the organisation required that staff fully document business activities. During the evaluation of applications, the documents generated and received were managed in a combination of physical files and digitally in the EDRMS. The EDRMS, the CRM and the physical file used the same unique application number to identify the records.

The unit also was also responsible for monitoring and reviewing incidents involving new organisms. ERMA New Zealand defined an incident as an event involving a new organism where the controls may not have been followed correctly or where adverse effects to human health and safety or the environment occurred. For selected incidents, an inquiry would be undertaken to identify if there were any issues with ERMA New Zealand systems or processes. The New Organisms unit kept details of all incidents and any inquiries undertaken to determine the effectiveness of the regulation of new organisms. This function was responsible for maintaining comprehensive details relating to incidents and inquiries in case further incidents or inquiries occurred and a complete history of activities was required.

The New Organisms unit was comprised of the Group Manager, the Science Manager, the Applications Manager, science advisors and support staff. The majority of the staff in the New Organisms unit had a scientific background, including experience in one or more fields relating to the risk assessment and management of new organisms. Between them, New Organisms staff had conducted postgraduate research in molecular biology, immunology and pathology, plant molecular biology, genetics, plant pathology and virology, mammalian physiology, mycology, entomology, ecology, zoology, conservation and biodiversity (Environmental Risk Management Authority, 2011). 


\subsection{Recordkeeping behaviours}

This case study focuses on recordkeeping behaviours demonstrated by the participants as they went about their daily work from the perspective of the two main functions of the New Organism's unit: considering applications, and monitoring and reviewing incidents relating to new organisms.

\subsubsection{Consider applications}

This section of the case study discusses in more detail the recordkeeping behaviours involved in three of these process steps: processing an enquiry from an applicant, formally receiving applications and evaluating applications. Table 4.1 outlines the entire the application process, although depending on the type of application the specific steps in the process varied.

Table 4.1: ERMA New Zealand application process

\begin{tabular}{|l|l|}
\hline \multicolumn{1}{|c|}{ Process } & \multicolumn{1}{c|}{ Description } \\
\hline $\begin{array}{l}\text { Contact from an } \\
\text { applicant }\end{array}$ & $\begin{array}{l}\text { The first contact with ERMA New Zealand was usually an enquiry. } \\
\text { As part of processing the enquiries, staff would help determine if } \\
\text { an application was required. Each enquiry was assigned a unique } \\
\text { enquiry number. }\end{array}$ \\
\hline Pre-application period & $\begin{array}{l}\text { The pre-application period began when applicants confirmed they } \\
\text { would submit an application. Applicants sent a draft application to } \\
\text { ERMA New Zealand for comment, ERMA New Zealand generated } \\
\text { a unique application number (e.g. ERMA200xxx) and assigned the } \\
\text { application to an advisor. }\end{array}$ \\
\hline $\begin{array}{l}\text { Formal receipt of the } \\
\text { application }\end{array}$ & $\begin{array}{l}\text { An application was formally received once a completed signed } \\
\text { physical and a digital copy were submitted and the application fee } \\
\text { was paid. }\end{array}$ \\
\hline $\begin{array}{l}\text { Public notification (for } \\
\text { notified applications } \\
\text { only) }\end{array}$ & $\begin{array}{l}\text { Some applications were required to be publicly notified. Notices } \\
\text { were placed on the ERMA New Zealand website, in the four major } \\
\text { New Zealand newspapers and in the Gazette informing the public } \\
\text { that the application had been received. This notification occurred } \\
\text { within ten working days of the formal receipt (unless a waiver has } \\
\text { been received) and the public had 30 working days to lodge } \\
\text { submissions. }\end{array}$ \\
\hline
\end{tabular}




\begin{tabular}{|l|l|}
\hline $\begin{array}{l}\text { Evaluation and review of } \\
\text { the application }\end{array}$ & $\begin{array}{l}\text { The advisors evaluated and reviewed the application and } \\
\text { submissions (where relevant) resulting in a report or a draft } \\
\text { decision. The risks and benefits of the substance or organism and } \\
\text { its proposed use were assessed in the document. }\end{array}$ \\
\hline $\begin{array}{l}\text { Hearing (for notified } \\
\text { applicants only) }\end{array}$ & $\begin{array}{l}\text { Where an application was publicly notified, a hearing might be } \\
\text { held, allowing those people who provided a written submission to } \\
\text { have an opportunity to present their submission in person. The } \\
\text { Decision-Making Committee used the hearings to obtain further } \\
\text { details. }\end{array}$ \\
\hline $\begin{array}{l}\text { Consideration by the } \\
\text { Cocision-Making }\end{array}$ & $\begin{array}{l}\text { The Decision-Making Committee met to consider the application } \\
\text { and decided whether to approve, not approve, or decline the } \\
\text { application, or whether more details were required before they } \\
\text { could make a decision. }\end{array}$ \\
\hline Notify the applicant & $\begin{array}{l}\text { Once a decision was made, ERMA New Zealand published a } \\
\text { summary of the decision and a copy of the decision document on } \\
\text { the ERMA New Zealand website. }\end{array}$ \\
\hline
\end{tabular}

\subsubsection{Enquiry to ERMA New Zealand}

Often, the first step to apply to introduce a new organism to New Zealand was an enquiry to ERMA New Zealand. Enquiries were initiated by members of the public, research organisations or tertiary institutions and could be made to an email address or by telephone, with New Organisms' advisors who were rostered on for specific times to manage responses to these enquiries.

Vignette 4.1 describes a New Organisms advisor, Janet, who was rostered on to respond to enquiries during the observation, registering details of an email enquiry from a member of the public into the CRM, a regular activity for all New Organisms advisors. Janet worked in the New Organisms open plan office area, alongside other New Organisms advisors. She had a number of notebooks, sticky notes and piles of paper on her desk. She had an offsite storage archive box behind her desk, which she explained she had recalled from the offsite storage provider to collate information for an OIA response. The enquiry she was working with was an email from a member of the public relating to the importation of a specific type of fruit tree into 
New Zealand. The process of registering the enquiry details into the CRM required the advisor firstly to create the enquiry entry in the CRM, then to save all email conversations to the appropriate location within the EDRMS as well as any supporting documentation. The records were linked digitally through the use of a unique enquiry number generated by the CRM, which was added as metadata to the items saved in the EDRMS.

\section{Vignette 4.1: Registering an email enquiry}

Janet opens the CRM and selects the option to create a new enquiry. An online form opens and she copies the subject line from an email in her Sent Items folder and pastes this into the enquiry description field. She clicks 'Save', which closes the enquiry form and as a result automatically generates a unique enquiry number.

Next, she resizes the EDRMS and Outlook windows open on her screen and she drags the email she sent in response to the enquiry and drops it into the location in the EDRMS for all enquiry documentation. A profile window opens displaying the metadata properties to be completed. She changes the first metadata property, which is a drop down box, from 'Document' to 'Email'; this changes the metadata properties to those that are email specific. She renames the title to include the recipient's name, the subject and the date she sent the email. Then she clicks 'OK', which closes the profile window and saves her sent email, along with the metadata, to the EDRMS. She opens the CRM enquiry form she previously created and copies the enquiry number and clicks ' $O K$ '. Returning to the EDRMS, she then pastes the enquiry number to the description field for the document she just saved. 
Next, Janet opens an email in her inbox, which includes a reference article as an attachment. She drags this attachment to the location in the EDRMS for enquiry documentation and a dialogue box opens, prompting her to complete the document properties. The title she gives this document is based on the full article title, which requires her to return to Outlook and open the pdf article; she returns few times to this pdf to check she is typing the title correctly. When all of the metadata properties are completed, she clicks 'Save'. She returns to the CRM enquiry form and she changes the enquiry status to completed.

This process to capture details of the enquiry into the CRM and to save the related attachment to the EDRMS took approximately 20 minutes, excluding the time that Janet spent composing her email response before the observation. This vignette highlights the time and effort necessary to complete this administrative task, especially when some of the details appear trivial. Much of this process involved Janet manually copying details and pasting it between the CRM and the EDRMS, including the enquiry number. If an error was introduced during this data entry process, some details might be lost.

Vignette 4.1 also illustrates where staff members made allowances for idiosyncrasies and limitations of the systems, for example, Janet created and saved the enquiry form in the CRM, and then she reopened and edited to copy the enquiry number as the CRM did not allow staff to copy this field until they saved the enquiry. This system limitation created the possibility of human error; if a staff member forgot to copy the enquiry number and paste it to this metadata property in the EDRMS, it would result in an incomplete record of the enquiry and with records 'orphaned' in the EDRMS. 
Some enquiries were more complex than others; potentially involving multiple interactions with members of the public as well as the use of multiple communication channels, with each interaction needing to be lodged by staff into the CRM. During his interview, Glenn explained:

\footnotetext{
If I'm dealing with an enquiry which in theory has a beginning and an end, all of the documents are saved in that name... labelled one to whatever, one to 20, and that's based on the chronological order. It means you end up with a lot of files called one or two, but then the string after that is different, so... you can slot them together, through the CRM because there'll be a CRM number associated with it, and that will give you a link to the CRM number, and you can pull up the rest.

(Glenn, ERMA New Zealand)
}

This convention, devised internally by the New Organisms team, ensured the single enquiry number linked all of the emails and the evidence of interactions with external parties and maintaining the sequence of these interactions. Maintaining the sequence of interactions was important to allow advisors to recreate interactions if necessary. The ability to present the enquiry interactions in the sequence they occurred was not available in either the CRM or the EDRMS systems and is an example of the team identifying a limitation of the system, developing an internal process and of staff consistently following the steps to make allowances for this system limitation.

\subsubsection{Formal receipt of an application}

When ERMA New Zealand received applications for new organisms, they assigned them an application number, different to an enquiry number. The application was acknowledged as formally received once applicants submitted a completed signed copy of the application in both physical and digital formats, and paid the application fee. Once an application was formally received, this started the period for ERMA New Zealand advisors to evaluate and review the applications. 
The second vignette describes Danielle managing a completed New Organism application form and an email about the payment of the required application fees as part of the process to receive that application formally. Danielle was an advisor, in a similar role to Janet. She described this role as:

\footnotetext{
I am an Environment Risk Advisor, and that means I deal with applicants and also write reports and things for the decision-making committees. And sometimes we have to deal with submitters and things like that. But mostly it's applicants or potential applicants that I deal with.

(Danielle, ERMA New Zealand)
}

Danielle had two piles of reference material on her desk; one was labelled 'to read', while the other was a small pile of journals from ERMA's internal library. Behind her was a small filing cabinet, which was open, showing that she had organised copies of previous decisions and legislation into folders.

\section{Vignette 4.2: Managing a completed New Organism application}

A paper version of the application form is laid out in front of Danielle on her desk. She is in the process of saving a digital version of the application to the EDRMS in the location for that application that support staff had previously created. An email from the ERMA New Zealand Finance team is open on one of her monitors, which she prints before saving it to the EDRMS using the preconfigured Colligo folders in her Outlook email account, allowing her to drag and drop the email into application folder in the EDRMS. An EDRMS metadata profile window pops up, and she changes the content type to email and renames the email to "Finance fm, application fee (2010 12 10)". She completes the metadata, including changing the status to final, the audience to internal, leaving the document type as blank and adding a 
keyword of correspondence. She also selects the year "2010/2011" from the drop down list. Once she has completed this and selected save, the metadata profile window closes and she gets up to collect the printed email from the printer.

Danielle then starts a new message to an ERMA New Zealand colleague, requesting the support staff member create a new physical file for this application. She opens the application document, which was attached to another email in her Outlook inbox and from here copies the details about the application, including the applicant's contact details, application number and application type. Once she sends this email, she lines up the paper version of the application and the printed email into a pile on her desk.

During the fieldwork and interviews, participants explained that since the introduction of the EDRMS, if the records were managed in the EDRMS system, it was deemed to be managed to sufficient level of care to be authoritative records. Accordingly, the main purpose of the physical files was to manage records that the agency had received only in print format. However, as this vignette demonstrates, staff continued to manage records in both physical and digital formats; in this vignette, an application form and related email. Danielle saved this email in both formats because she deemed it to be significant, as she did not follow this approach for all emails that she saved to the EDRMS. Danielle determined whether to capture records in both formats based on her own interpretation of the significance of the content. 
The process of registering enquiries and applications from external parties was a task performed by New Organism advisors on a regular basis throughout their working days. While the CRM and the EDRMS systems provided the mechanisms to register these enquiries, it was the responsibility of each advisor to collect the details and populate the systems with details about each enquiry-related interaction. However, not all enquiries were email-based correspondence. The CRM allowed for staff to enter details about a telephone interaction and complete a description field; however, for those enquiries that were not email-based, it was sometimes difficult for participants to know what specific details to capture. One participant asked members of the public who contacted her via the telephone to email their enquiry as she explained below:

\footnotetext{
Most of the time what I get people to do is email, 'so how about you email me and actually write down exactly what you want?' and that way we've got it formally down. I don't like having un... writing down my interpretation of what happened. I like to actually have some solid, concrete text.

(Kelly, ERMA New Zealand)
}

While Kelly described how she preferred dealing with enquiries that the enquirer documented, Vignette 4.1, Vignette 4.2 as well as other fieldwork conducted with the New Organisms advisors, suggest there were multiple opportunities for the advisors to use their discretion when saving enquiry-related records. Ultimately, advisors decided whether to save the interaction to the CRM, when to save the records to the system, what details from the interaction to save, the level of details captured regarding the interaction and whether the record was deemed sufficiently significant to be captured in both physical and digital formats. If advisors did not register their interactions with external parties, there would not be any documented evidence of that interaction created or captured by the agency. 


\subsubsection{Evaluation of applications}

After an application was formally received, one of the subsequent steps was for ERMA New Zealand advisors to evaluate and review the application, resulting in a draft decision report. The decision report assessed the risks and benefits of the organism and its proposed use. This report was made available to the DecisionMaking Committee, who decided whether to approve, not approve, decline the application, or whether more details were required before the decision could be made.

The following vignette describes Glenn as he prepared a draft decision report in response to an application to import and release up to eleven species of dung beetles to overcome the adverse effects caused by animal dung in New Zealand pastures. Glenn described his role as:

I am Science Manager, New Organisms. So in that role, I overview the science that goes into applications and assessing applications. And I manage a small group of advisors. (Glenn, ERMA New Zealand)

The New Organisms team worked in an open plan environment. Glenn's desk neighboured other New Organisms advisors, with high dividers on both sides and a view out to the Wellington Harbour. He had two monitors on his desk and some reference books and journals around the desk as well as large piles of reference articles around his work area. He also had a bookshelf beside his desk where the books were laid out in piles. There was an offsite storage archive box on the floor and another under his desk, next to a small credenza.

\section{Vignette 4.3: Editing a draft decision report}

Glenn is editing a draft decision report in Microsoft Word; he is using 
the 'track changes' feature to manage the changes as he makes them. He has a digital read-only copy of the application open on his second monitor and the first page of the application is printed and sitting on a tray above his keyboard.

As he is editing the report, he opens Google and conducts a search. From the results that are returned, he opens a pdf document and after a brief read, he saves this document to a folder on his $\mathrm{H}$ Drive called 'My References' and opens the document on his second monitor. He refers to this article as he drafts two paragraphs in the report.

He opens a new Google window and conducts another search. He selects a link from the search results, opens it, and quickly reads the web page.

He quickly brings up Outlook and writes a brief email to an internal colleague. When he clicks send, he receives a message that he has reached the limit of his email account and cannot send the email. The message offers the option to empty his deleted messages folder, which he does and, consequently, he can send the email.

After sending the email, he continues working on the report. Glenn conducts another Google search but doesn't appear to receive the results he desired, so he turns to his bookshelf for a book and at the same time, makes a sarcastic comment out loud about his "wonderful filing system". He found the book he was looking for immediately.

After referring to a specific section in the book, he adds some content to the report and adds the book's reference details. Glenn now conducts some further Google searches. The observation concludes as Glenn 
continues working on the report.

During this observation, Glenn saved a reference document he referred to in the decision report he was preparing to his personal drive, referred to within ERMA New Zealand as the H Drive. Glenn also demonstrated the use of his personal drive to store decision-related documents during a subsequent observation (Vignette 4.4), demonstrating that not all records he created and used when evaluating New Organisms applications and preparing the decision reports, were saved to the EDRMS.

\section{Vignette 4.4: Saving documents to personal drive}

Glenn begins reading an email about an external collaborative group meeting scheduled for February. He opens his Outlook calendar on his second monitor and creates a new meeting request to himself for the particular date. He then checks in his calendar for availability on the days either side of this meeting and replies to the message confirming his availability.

He saves the email in "My Work > Supporting Decision Making > Research", renaming it as part of the save process.

At this point, he locates a personal storage device from his credenza drawer, puts it into his computer and creates a new folder, “My Work > WRA Paper". Into this new folder, he adds the attachment from the email while saying, "that's my homework for the weekend." 
Vignette 4.3 and Vignette 4.4 describe how Glenn used personal information repositories, external to the EDRMS, to manage some work-related records. He explained that he used the personal drive on his work computer for current draft documents before saving the final versions in the EDRMS:

\footnotetext{
I have got a parallel filing system on my h drive where I tend to put the stuff... it's often where I'll sort material before it goes into the main files. Because I find that I don't want to end up filing stuff in the permanent files that turn out to be ephemeral, so I find it easier to let it accumulate in one place, organise it and then drop it into the filing system.

(Glenn, ERMA New Zealand)
}

Glenn explained that he tended to use his 'My Work' folder as a reference for records that he was working on, while he would capture records directly related to applications and final versions of records within the EDRMS. This practice is an example of how staff applied the discretion available to them in how they used the ICTs.

\subsubsection{Monitor and review the enforcement of the HSNO Act}

In addition to considering applications, the New Organisms unit was also responsible for monitoring incidents involving new organisms and reviewing the enforcement of the HSNO Act. This responsibility was smaller than that of considering applications for new organisms; involving just one staff member, Sharon. Sharon was responsible for collating details on any incidents involving new organisms and for managing the records used to review the enforcement of the HSNO Act. This section of the case study discusses the recordkeeping behaviours of Sharon as she went about her daily work to monitor and review the enforcement of the HSNO Act.

\subsubsection{Monitor new organisms' incidents}


ERMA New Zealand registered any incidents involving new organisms in an internal database to monitor the effectiveness of the regulatory system and the appropriateness of the controls set as part of the approval. Incidents relating to new organisms took the form of incidents involving zoo animals, new plants, new microorganisms and new animals held within invertebrate, vertebrate or field test containment facilities. The incident data was either collected by monitoring compliance with the controls that were attached to the approvals, reported to ERMA New Zealand by enforcement agencies or identified through media monitoring. Summaries of all incidents were published in the ERMA New Zealand Annual Report to Parliament and in the Annual Monitoring Report. All organisations involved in any incidents were informed before the release of these publications.

Sharon had been in this role for a number of years and did not have a scientific background like the New Organisms science advisors. Sharon was the primary point of contact in relation to New Organisms incidents, which could relate to current or historical events; she could be contacted by members of the public with a specific question, staff from containment facilities such as zoos or research facilities as well as staff from other agencies. During the observations, Sharon's main working relationships were with specific individuals from the Ministry for the Environment and the Ministry of Agriculture and Forestry (now part of the Ministry of Primary Industries). Sharon also communicated with staff from organisations involved in new organisms incidents, liaising with them for any additional details regarding the incident and before the release of the annual monitoring publications. During one observation period, Sharon prepared summaries of recent incidents to add to the internal incident database. It was her responsibility to complete a Microsoft Word form, which once completed she provided it to another member of the New Organisms unit, who added the details from the form into the incident database. This database was used to generate a report, which was printed and filed at the front 
of the "Incidents" physical file in a clear pocket and used in the preparation of the annual monitoring reports. In this case, the incident concerned a red panda that had escaped from its enclosure at Wellington Zoo. The red panda was recaptured the same day, and after a review of the incident, it was thought that weight of rain-water plus the weight of the red panda bent some tree branches down sufficiently for the red panda to jump out of the enclosure. As a result, the zoo pruned the vegetation in the enclosure.

\section{Vignette 4.5: Preparing summaries of recent incidents}

With the "Incidents" physical file open in front of her, Sharon navigates through the EDRMS to open the blank Incidents form, which is a Word document. Sharon spends a couple of minutes populating the form; she refers to documents in the physical file and documents in the EDRMS to confirm names, dates and details are correct.

Once the form is complete, she selects "Save As", navigates through the EDRMS to save the document in the appropriate digital location and renames the document to "Incident Category Classification Wildlife Reserve Non Compliance Perimeter Fence". Once saved, she prints the completed form.

There are two more incident forms, which Sharon completes in the same manner. Once she completes the forms, she walks to the printer and collects the three printed forms and takes them to a colleague's desk, where she leaves them. Back at her desk, Sharon sends this same colleague an email with links to the three saved forms in the EDRMS. 
Vignette 4.5 describes a situation where records were manipulated multiple times, both between colleagues and between systems. The forms were saved digitally in the EDRMS and printed; they were then to be re-entered digitally into other ICTs by another staff member to be printed to manage in the physical file. As already mentioned with the introduction of the EDRMS, if the records were managed within the EDRMS, the agency deemed that record to be managed to sufficient level of care to be an authoritative record and therefore not required to be printed. However, as Sharon explained during her interview, she preferred referring to physical records rather than digital records as she wanted to browse the incident records in the physical file. In this situation, staff had specific personal preferences for retrieving records; meeting these personal preferences introduced additional steps in the processing of these records.

Vignette 4.5, along with the other vignettes in this Chapter, illustrate the importance that the participants placed on completing the metadata profile for each item, in particular the title. As Sharon explained in her interview:

\footnotetext{
You have to not be slap-dash and you have to think carefully about... you know, in terms of titles. And there are I suppose formalities in terms of how you write your title and there are formal ways of... in terms of who you received the information from, what the information is about it, etc. Although the metadata does guide you in terms of keywords, dates etc. (Sharon, ERMA New Zealand)
}

For the most part, ERMA New Zealand staff used the EDRMS metadata for searching for items and to identify specific records when they were browsing the EDRMS document libraries.

\subsubsection{Review the enforcement of the HSNO Act}


Sharon was also responsible for collating and managing details used in the annual review of the enforcement of the HSNO Act. This culminated in the Annual Monitoring Report, which included four parts:

- a commentary on the overall performance of the HSNO Act in that financial year,

- a summary of the major conclusions of an investigation, different each year, on the impact of the HSNO Act in New Zealand,

- a presentation of statistics drawn from data from a range of financial years, and

- analysis of any incidents by location, severity and impact on people and the environment.

The following vignette, Vignette 4.6, describes Sharon preparing communications with staff from Scion, a Crown Research Institute based in Rotorua, specialising in forestry and wood-related research, science and technology development. Sharon had a field test report on her desk that she had received from Scion, which included handwritten comments, a signature and a date from one of the ERMA New Zealand New Organisms' managers, confirming they had reviewed the report. "This covers my arse that they were reviewed," she says. This vignette describes Sharon writing a confirmation letter that ERMA New Zealand received and accepted the report from Scion, which would be used in the development of the Annual Monitoring Report. To do this, she started by opening a letter sent in 2008 .

\section{Vignette 4.6: Preparing communications with external organisation}

Sharon opens the 2008 letter and saves a copy to her desktop, renaming 
the file to "Simpson I to re 2010 annual report GMF [number]/GMF [number]". Then she uploads the document into the EDRMS and reviews the automatically populated metadata properties without making any changes. On clicking save, an EDRMS message appears that the " /" in the filename is invalid and the file cannot be saved, so with an audible sigh, she renames it without the "/".

With the file saved in the EDRMS, she double clicks to open it. The content of the letter was retained from the original 2008 letter; she updates the date to today's date and reviews the remainder of the letter, without making any further changes. She saves the file and prints it. Once printed, she walks to the printer, signs the letter and scans it to generate a digital copy. Returning to her desk, she navigates to the shared folder containing scanned digital files, "Is this my one?" she asks out loud, looking at a long list of files with system-generated names. Once confirmed that it was the letter she scanned, she saves the file to her desktop, renaming it by suffixing the file name with "copy".

Sharon then uploads the scanned document into the EDRMS and completes the metadata before printing this document, which she collects from the printer. In the physical file in front of her, she puts the report from Scion in a clear plastic sheet along with the printed version of the signed letter. She puts the original signed copy of the letter into an envelope, which she addresses before getting up from her desk and putting it in the shared outward post tray.

She then starts a new email message to the Scion staff member and attaches a copy of the scanned, signed letter and informs them that she has posted a physical copy. She carbon copies the email to named 
Ministry of Agriculture and Fisheries staff.

Once she sends the email, she uses Colligo to drag and drop the sent email into the relevant EDRMS folder, renames the email, and updates the metadata indicating that the version is final. She also prints the sent email which she places in the physical file.

Vignette 4.6 illustrates Sharon's comprehensive approach to capturing and organising correspondence records. Every step of the process Sharon followed to confirm that ERMA New Zealand had received a document from another organisation she duplicated to ensure that all records were available in both the physical and the digital recordkeeping environments, regardless of their original format.

This vignette illustrates the differences in how Sharon creates and captures the two different formats of records. Sharon creates the initial letter by saving an existing digital record as a new item and then editing it, thus saving her considerable time than if she had to recreate the correspondence from a physical record. The capture process of the physical letter is considerably less time-consuming as Sharon puts the report from Scion in a clear plastic sheet along with the printed version of the signed letter. In comparison, capturing the digital format of the scanned letter in the EDRMS requires Sharon to upload the scanned document into the EDRMS and complete the EDRMS metadata profile. To capture that the scanned document has been emailed to the Scion and Ministry of Agriculture and Fisheries staff, Sharon uses Colligo to drag and drop the sent email into the relevant the EDRMS folder, renames the email, and updates the metadata indicating that the version is final; she prints and files a physical copy as no metadata is required. 
Vignette 4.6 also demonstrates the continuing importance of traditional communications channels within ICT-enabled public agencies; in this example, although Sharon could only email the Scion staff, she also sends a physical letter to them, while she also emails to Ministry of Agriculture and Fisheries staff.

\subsection{Discussion}

Drawing on concepts explored within the analytical framework, two significant themes were identified during the fieldwork at ERMA New Zealand:

- that front-line staff at ERMA New Zealand have the discretion to manage public records according to their personal understanding of their recordkeeping responsibilities; and

- that staff manage records within a hybrid digital-physical recordkeeping environment, which impacts how they create, manage and use records.

\subsubsection{Discretion}

ERMA New Zealand staff were responsible for deciding if, and when, to create a public record, what format to capture the record in, where to save the record, and as a consequence of where they stored the records, who would have access to it and how long the agency retained it. The ICT Manager and the Corporate Services Manager both stated that the agency did not monitor staff members' recordkeeping behaviours for compliance with the responsibilities set out in the ERMA New Zealand records management policy. ERMA New Zealand purposefully configured the EDRMS to allow staff certain aspects of discretion, as Julie $S$, the Corporate Services Manager responsible for recordkeeping and information management, explained: 
If you put something in that is so rigid that it makes it too difficult they will not do it [...] So it is a fine line - in allowing people if they want to put more information in to do that, getting the right amount of minimum information in there so that you can find something, so that anyone can find it, but not making it too onerous. So, um, I prefer and we tend to operate on a principle based system as an organisation and in terms of records management, um I think I would hate to see us going down the line of you know you must fill-in all of these boxes before you can file the email or whatever it is. (Julie S, ERMA New Zealand)

During the interviews, participants explained that they were confident in their abilities to identify public records and understood they had responsibilities for managing these public records. Participants mentioned the need to create and capture records to provide evidence that activity occurred or that they had obtained the necessary approval:

So if it comes back and bites me... I can say I've actually notified everyone.

(Sharon, ERMA New Zealand)

Participants also used their discretion to create records in both digital and physical formats, even when not required by the agency and although it increased the effort required for the staff members. For some staff members, duplicating records in both digital and physical formats was only observed when they deemed the records to be of particular importance, again using their discretion to determine this; while for other participants, engaging with the organisational recordkeeping systems was limited to capturing final versions of documents. Glenn, who was observed using his personal drive as well as personal storage devices to manage application-related records, explained that his concerns about using the EDRMS related to version control:

If you save minor versions into the system, you can't get them out; it would be ok if you could delete things but you can't. 
Discretion was also evident in the specific ways that participants engaged with the ICTs. As Glenn explained, he was concerned about the functionality available within the EDRMS whereby staff could configure options to be emailed based on certain actions, such as when a particular document was edited; "busy bodies use them to keep track of things". He explained that as some staff members had alerts set on certain document libraries, he purposely would not save anything into these locations until the record was finalised; allowing him to retain control over when people had access or visibility to the records.

\subsubsection{Managing records in a hybrid environment}

The hybrid digital-physical recordkeeping environment at ERMA New Zealand impacted how staff created, managed and used records. ERMA New Zealand employees regularly created and managed digital records such as reports and memos, spreadsheets and emails. They also created, received and managed physical records, such as letters signed by ERMA New Zealand staff and sent to external parties, physical copies of applications, faxes and letters received by the agency. As described in the vignettes (particularly Vignettes 4.2 and 4.6), some ERMA New Zealand employees managed the same records in both digital and physical formats and attempted to mirror the contents of the digital and physical locations.

This hybrid recordkeeping environment was exacerbated at ERMA New Zealand by the use of multiple historical recordkeeping systems, including two separate historical shared drives and the EDRMS, as well as the physical filing system. ERMA New Zealand managed each of these recordkeeping systems independently, had no intention to merge these separate recordkeeping systems, and in the case of the two 
historical shared drives, made them read-only so staff could not add new content, but all staff could read and access the existing contents.

As well as the efforts of some staff to save the same records in both the digital and the physical recordkeeping systems, this hybrid recordkeeping environment also introduced additional effort for staff when they were searching for and retrieving records:

If you've got to look in two places, then you're going to miss stuff.

(Glenn, ERMA New Zealand)

This situation was likely to be exacerbated as whether records were saved in a particular format was determined by the individual advisor and there were no consistent approaches followed by the team members.

Some participants indicated that it was not always obvious what documents they needed to save to the recordkeeping system or where to store them, and this was particularly true where the record was not directly related to an enquiry or application. Where participants did not know what to file or where to store it, they indicated this sometimes lead to situations where they saved records outside of the recordkeeping systems, on hard drives or personal drives, as described in Vignette 4.7:

\section{Vignette 4.7: Identifying locations to store digital records}

"What do I do with this?" Janet asks out loud as she looks at a folder called 'Survey' saved on her desktop.

The folder includes different versions of survey questions and findings report as well as versions of data analysis spreadsheets and the raw data 
for a client satisfaction survey. In addition to these files, Janet has a number of related email threads, which she has stored in her email account. The final report, completed several months prior, provides a summary of the survey findings and recommendations for $\mathrm{New}$ Organisms and ERMA New Zealand to improve the service provided to individuals and organisations that interact with them.

She opens a document library in the EDRMS; "there is so much stuff in this library, I don't want to add the survey documents here. It's so overwhelming".

The fieldwork suggested a varying degree of trust towards digital records and an aversion to risk amongst the participants. The EDRMS was still relatively new for many staff, and particularly for those who had been employed by the agency for a significant length of time, represented a significant change from their previous ways of working.

Furthermore, within this hybrid recordkeeping environment, staff replicated traditional physical recordkeeping approaches in the digital environment, without taking full advantage of the digital environment. For example, Sharon indicated that she preferred browsing the physical incidents file as she was able to store significant records in the front of the file, ensuring summaries of incidents were immediately visible. However, she had not attempted to replicate this approach within the EDRMS document libraries. Staff also relied on physical records for particular situations, for example, Kelly explained that she felt it was necessary at times to create a memo or file note signed by a manager to demonstrate that she had management support for her actions: 
Especially when we need sign-off, so I want managers to say I think you should do this, you guys agree with me please and so that's quite important... Especially as certain things have to be documented as part of the process. And that's how we... that's the easiest way to do it. You can kind of do it using email if you really wanted to, but it's not the best. It's best to get a signed... a sign-off.

(Kelly, ERMA New Zealand)

Interestingly, the ICT Manager described the EDRMS as "light-weight", explaining that ERMA New Zealand had not enabled some of the functionality traditionally available in an EDRMS in an effort to encourage widespread use of the system by staff:

There are, I think, some more things that need to be switched on with the EDRMS as it stands at the moment, it's very light-weight. We don't have versioning; we don't have check in / check out. So... it's very easy to use because that's not there but that needs to be switched on to avoid some of the issues with people editing the same document and versioning, they get a bit confused.

(Richard, ERMA New Zealand)

Overall, ERMA New Zealand staff worked within a recordkeeping environment where scientific records and business administration records were managed alongside customer relationship records, both in the physical recordkeeping environment and within, what was at the time, a relatively recent implementation of an EDRMS alongside multiple historical shared drive environments. This hybrid recordkeeping system led to duplication of effort by staff and records across digital and physical environments as staff attempted to ensure the records were easily retrievable and accurate. It is also highlighted the limited use of some EDRMS functionality by the participants (for example advanced searching based on metadata, control of multiple versions of the same record, or digitally linking related records), as staff tended to apply physical recordkeeping approaches that they were familiar with. 


\section{Recordkeeping behaviours at Te Puni Kōkiri}

This chapter describes the second case study conducted at one regional office of Te Puni Kōkiri, the Ministry of Māori Development, between May and August 2011. This chapter introduces Te Puni Kōkiri's purpose, core functions and organisational structure, with a specific focus on the role of the regional offices. This section describes the participants' recordkeeping behaviours, drawing on details provided during the interviews conducted with the regional staff.

\subsection{Te Puni Kōkiri}

Te Puni Kōkiri, under its various titles, played a number of roles within New Zealand over the past 160 years (Luxton, 2008, p. 5). The role of this agency has changed from a focus on communicating between Māori and Pākehā cultures to assisting in the development of farms, to providing welfare, and more recently returning to a developmental approach and the provision of a Māori perspective for the government (Luxton, 2008, p. 5).

At the time of the fieldwork, Te Puni Kōkiri's principal duties, as defined by the Ministry of Māori Development Act 1991, were to promote increases in Māori achievement across the four key social and economic areas of education, training and employment, health, and economic resource development (Ministry of Māori Development Act, 1991). Te Puni Kōkiri was also responsible for monitoring and liaising with agencies that provided services to, or for, Māori to ensure the adequacy of those services (Te Puni Kōkiri, 2011, p. 12). Te Puni Kōkiri was a policy-led ministry tasked with advising its Ministers and the government on the Crown's relationship with Māori, including opportunities and risks inherent in that relationship. Te Puni Kōkiri's advice was focused on improving outcomes for Māori 
through whānau ${ }^{3}$-centred approaches and Treaty of Waitangi-based partnership relationships between the Crown, hap $\overline{\mathrm{u}}^{4}$ and $\mathrm{iwi} \mathrm{i}^{5}$, placing it in a unique position within New Zealand central government (Luxton, 2008, p. 5).

To carry out its core roles and functions, Te Puni Kōkiri combined a head office of policy, programme coordination and support services personnel with a network of eighteen offices, including ten regional offices, across six regions. The organisational structure consisted of:

- The Chief Executive's Office

- Policy wāhanga ${ }^{6}$

- Relationships and Information wāhanga, including the ten regional offices

- Support Services wāhanga

- Whānau and Social Policy wāhanga

\footnotetext{
${ }^{3}$ Whānau is defined as "extended family, family group, a familiar term of address to a number of people - the primary economic unit of traditional Māori society" by the Māori Dictionary (online) at www.maoridictionary.co.nz (accessed 1705 2017).

${ }^{4}$ Hapū is defined as "kinship group, clan, tribe, subtribe - section of a large kinship group and the primary political unit in traditional Māori society. It consisted of a number of whānau sharing descent from a common ancestor, usually being named after the ancestor, but sometimes from an important event in the group's history. A number of related hapū usually shared adjacent territories forming a looser tribal federation (iwi)" by the Māori Dictionary (online) at www.maoridictionary.co.nz (accessed 1705 2017).

${ }^{5}$ Iwi is defined as "extended kinship group, tribe, nation, people, nationality, race - often refers to a large group of people descended from a common ancestor and associated with a distinct territory" by the Māori Dictionary (online) at www.maoridictionary.co.nz (accessed 1705 2016).

${ }^{6}$ Wāhanga is translated as "section, chapter, division, part, share, place, faculty, semester, compartment" by the Māori Dictionary (online) at www.maoridictionary.co.nz (accessed 1705 2017).
} 
At June 2011 when the fieldwork was conducted, Te Puni Kōkiri employed 336 fulltime equivalent staff; seventy percent of whom were Māori (State Services Commission, 2011, pp. 23-24).

\subsubsection{Information management at Te Puni Kōkiri}

As a government agency, Te Puni Kōkiri was required to comply with New Zealand recordkeeping related legislation. Te Puni Kōkiri's statutory roles and responsibilities were prescribed by the Ministry of Māori Development Act 1991; in addition, Te Puni Kōkiri was responsible for administering 29 Acts of Parliament as well as a collection of regulations (Ministry of Justice, 2012, pp. 67-68). However, these Acts contained no specific recordkeeping responsibilities in addition to, or significantly different, to those outlined within the PRA.

A records management policy was in place within Te Puni Kōkiri. Staff interviewed were aware to some extent that an information and records management policy existed and indicated they could find it on the Te Puni Kōkiri Intranet if they had any questions regarding their obligations, although they did not elaborate on their specific responsibilities for managing records as defined within this policy.

All new staff received training as part of the Te Puni Kōkiri induction programme. One of the participants, Jessica, provided inductions to new staff members joining the regional office; the induction comprised of some hands-on training on how to use the basics of the EDRMS and the funding management system. Jessica also assisted new staff and answered any technical questions as required, or directed them to the Te Puni Kōkiri IT helpdesk for further assistance:

Well, they [new staff members] get ten-minute training from them here [pointing in the direction of Jessica, the Administrative Assistant]. At the first opportunity, um [...] We don't change staff that often. 
(Whetu, Te Puni Kōkiri)

The Te Puni Kōkiri head office also provided recordkeeping support to the regional offices. This was delivered in the form of face-to-face training conducted by a trainer who travelled to each regional office quarterly throughout the year and provided instructions on how to the EDRMS and the funding management system.

\subsubsection{Information technology at Te Puni Kōkiri}

At the time of the fieldwork, Te Puni Kōkiri operated an EDRMS that was available throughout the organisation, including at all of the regional offices, to manage digital business-related documents. Te Puni Kōkiri implemented the EDRMS, from Open Text, throughout the organisation between 2003 and 2004.

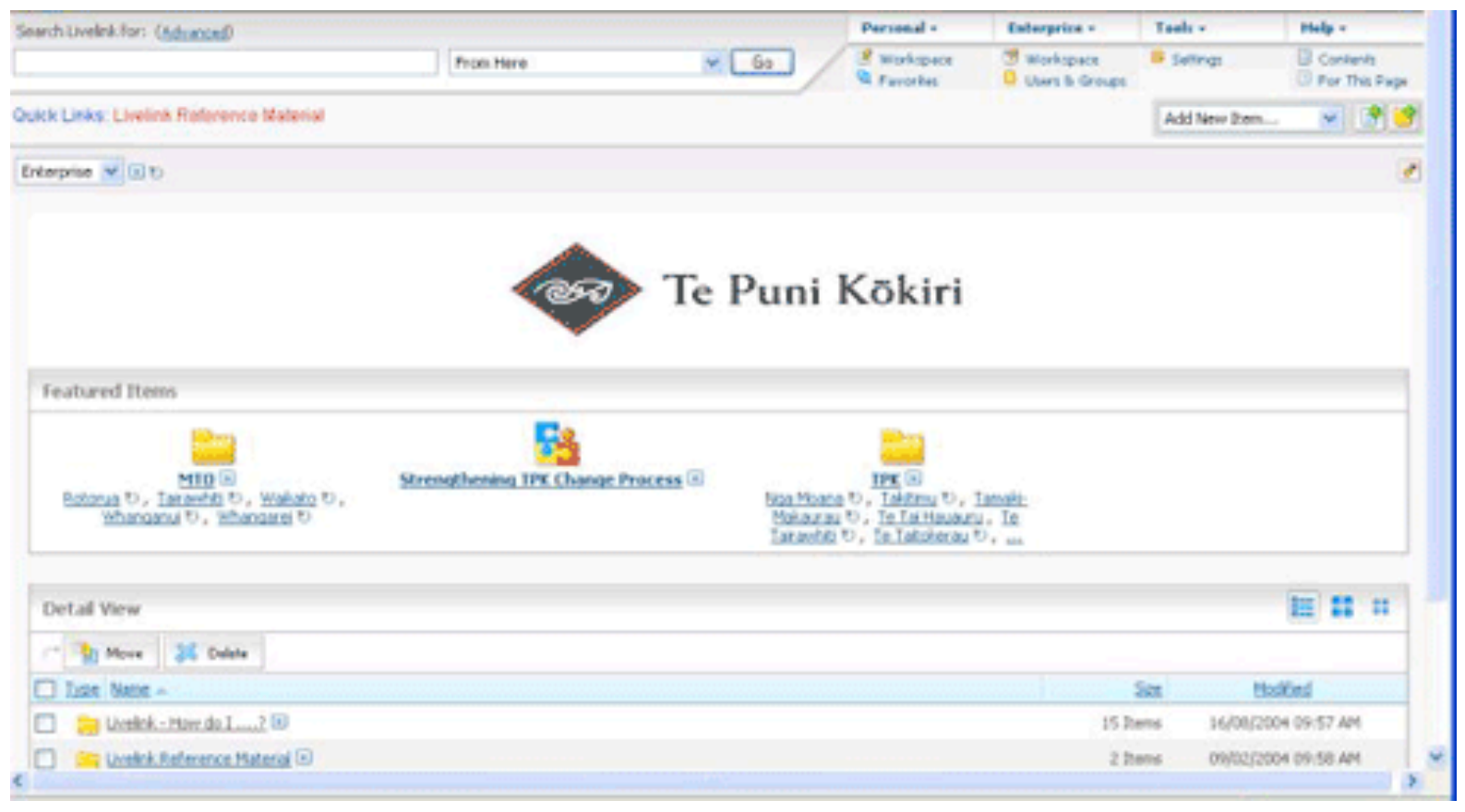

Figure 5.1: The EDRMS homepage (Department of Internal Affairs, 2005)

The EDRMS was structured according to Te Puni Kōkiri's six business functions: 
- Policy development: Contains all documentation created or received during activities and transactions relating to the development of policy. This functional area is structured by work streams or programmes.

- Relationship management: Activities relating to the development and management of relationships with Māori.

- Investing and intervening: Contains all documentation created or received during activities and transactions relating to investments from the Māori Potential Fund, and other funding initiatives.

- Monitoring and evaluation: Contains the inputs and outputs of activities and transactions undertaken in the course of monitoring compliance, entities and State Sector outcomes, as well as evaluating programmes and outcomes, and maintaining an internal audit programme.

- Strategic management and accountability: Records relating to Te Puni Kōkiri strategy and planning undertaken by the executive and senior management teams.

- Te Puni Kōkiri management: Records relating to the ongoing administration and management of Te Puni Kōkiri including personnel, financial management, property and equipment management, information management, communications, Ministerial, and legal records (Te Puni Kōkiri, 2016, p. 4).

The EDRMS used metadata to assist in the management of documents. Since 2008, every item saved to the EDRMS required metadata to be completed at the point of capture, some of which were mandatory, meaning the record could not be saved 
until these fields were populated, while other metadata properties were optional (Te Puni Kōkiri, 2016, p. 4). Te Puni Kōkiri training material explained that the primary purpose of metadata was to add the discovery and retrieval of records within the EDRMS; therefore, the more metadata applied, the higher the chances of authors, and other staff, discovering, and re-discovering, the records (Te Puni Kōkiri, n.d.).

Table 5.1: The EDRMS document metadata (Te Puni Kōkiri, n.d.)

\begin{tabular}{|c|c|}
\hline Metadata Property & Details \\
\hline $\begin{array}{l}\text { Author - Te Puni } \\
\text { Kōkiri }\end{array}$ & $\begin{array}{l}\text { Search for Te Puni Kōkiri staff members to identify those responsible } \\
\text { for creating the content }\end{array}$ \\
\hline Author - Other & Free text field to identify those responsible for creating the content \\
\hline Subject & $\begin{array}{l}\text { Required field. A drop-down list of topics e.g. aquaculture, resource } \\
\text { management. }\end{array}$ \\
\hline Subject Geographic & $\begin{array}{l}\text { A drop-down list of geographic regions and locations e.g. Waikato } \\
\text { region. }\end{array}$ \\
\hline Government Priority & $\begin{array}{l}\text { A drop-down list of current government key priorities e.g. economic } \\
\text { transformation. }\end{array}$ \\
\hline $\begin{array}{l}\text { Te Puni Kōkiri } \\
\text { Priorities }\end{array}$ & $\begin{array}{l}\text { A drop-down list of Te Puni Kōkiri priorities as defined in the } \\
\text { Statement of Intent e.g. maximising Māori assets. }\end{array}$ \\
\hline Priority Area & $\begin{array}{l}\text { Required field. Staff members select from a drop-down list of top } \\
\text { areas e.g. commercial enterprise. }\end{array}$ \\
\hline Priority Approach & $\begin{array}{l}\text { Required field. Staff members select from a drop-down list of } \\
\text { methods of approaches employed towards achieving work in priority } \\
\text { sectors e.g. asset management. }\end{array}$ \\
\hline Date Created & $\begin{array}{l}\text { Required field. Drop-down lists to record the creation date of the } \\
\text { document e.g. } 01062007 .\end{array}$ \\
\hline Document Type & $\begin{array}{l}\text { Required field. A drop-down list of known forms of documents e.g. } \\
\text { report, file note, correspondence. }\end{array}$ \\
\hline File Number & $\begin{array}{l}\text { Free text field for the file classification number of the physical file to } \\
\text { which a digital document relates e.g. NR } 5030 \text {. }\end{array}$ \\
\hline Security & $\begin{array}{l}\text { Required field. A drop-down list of security classifications. The } \\
\text { default value is 'none'. }\end{array}$ \\
\hline Access Status & $\begin{array}{l}\text { Required field. A drop-down list to facilitate public access to } \\
\text { documents deemed ready and appropriate e.g. published. }\end{array}$ \\
\hline
\end{tabular}


The Microsoft Office suite, including Word, Excel, PowerPoint, and Outlook, were used extensively by participants during the observations for staff; each of which integrated with the EDRMS. The integration of these systems allowed the automatic capture of some metadata, including specific required metadata properties; for example, the system automatically populated the staff member's name in the Author metadata property.

Staff also regularly used a funding management system, which was designed to manage all application and funding details. This system was configured to alert staff when milestones and funding reporting requirements were pending. Staff members used the funding management system to maintain contact details of external parties and to capture significant interactions (e.g. phone discussions, meetings, possible initiatives) regarding funding opportunities and applications. The funding management system was not integrated with the EDRMS.

\subsubsection{The regional offices}

Te Puni Kōkiri's ten regional offices provided a direct connection to iwi, hapū, whānau and Māori communities and offered the first point of call for enquiries about developing Māori communities, organisations or businesses. Te Puni Kōkiri assigned the regional offices with responsibilities for local-level facilitation and brokerage between Māori, the public, private and voluntary sectors, local-level consultation and issues reporting, and local-level management of the Ministry's social, cultural and economic development programmes and initiatives (Te Puni Kōkiri, 2011, p. 12). The regional offices provided support for entities applying to the Māori Potential 
Fund, which was designed to enable outcomes-based investments to help realise Māori potential.

One regional office participated in this research. Within this office, the Regional Director managed ten staff. The majority of personnel at this office were kaiwhakarite ${ }^{7}$, also known as regional facilitators or advisors. One of the participants described this role as:

It means, um, the one who works on the ground, has direct contact with our people, our clients.

(Whetu, Te Puni Kōkiri)

Kaiwhakarite responsibilities included specific accountabilities and deliverables relating to delivery of a work programme, stakeholder relationship management, capability development, investment advice and management, as well as information sharing (Te Puni Kōkiri, 2014). The information sharing responsibility specifically related to assisting to gather, maintain and disseminate information within the rohe ${ }^{8}$, manage the flow of information between stakeholders and the other wāhanga for programme evaluation and programme development, contribute to maintaining records of iwi, hapū and whānau and organisations, and provide regular briefings for the Regional Director on key issues and themes.

The regional office operated in an open plan work area. In addition to the open plan work area, there were a number of meeting rooms and a small kitchen area. In the middle of the office, there were large filing cabinets, which held current physical records relating to the work of the regional office. The regional office stored older,

\footnotetext{
${ }^{7}$ Kaiwhakarite is translated as "organiser, negotiator, agent, representative" by the Maori Dictionary (online) at www.maoridictionary.co.nz (accessed 1705 2017).

${ }^{8}$ Rohe is translated as "boundary, district, region, territory, area, border (of land)" by the Maori Dictionary (online) at www.maoridictionary.co.nz (accessed 1004 2017).
} 
non-current, physical files in the basement of the building. Jessica explained how for specific programmes of work involving contracts, she checked there was a signed, physical copy in the local file before sending the original signed contract to the Head Office in Wellington.

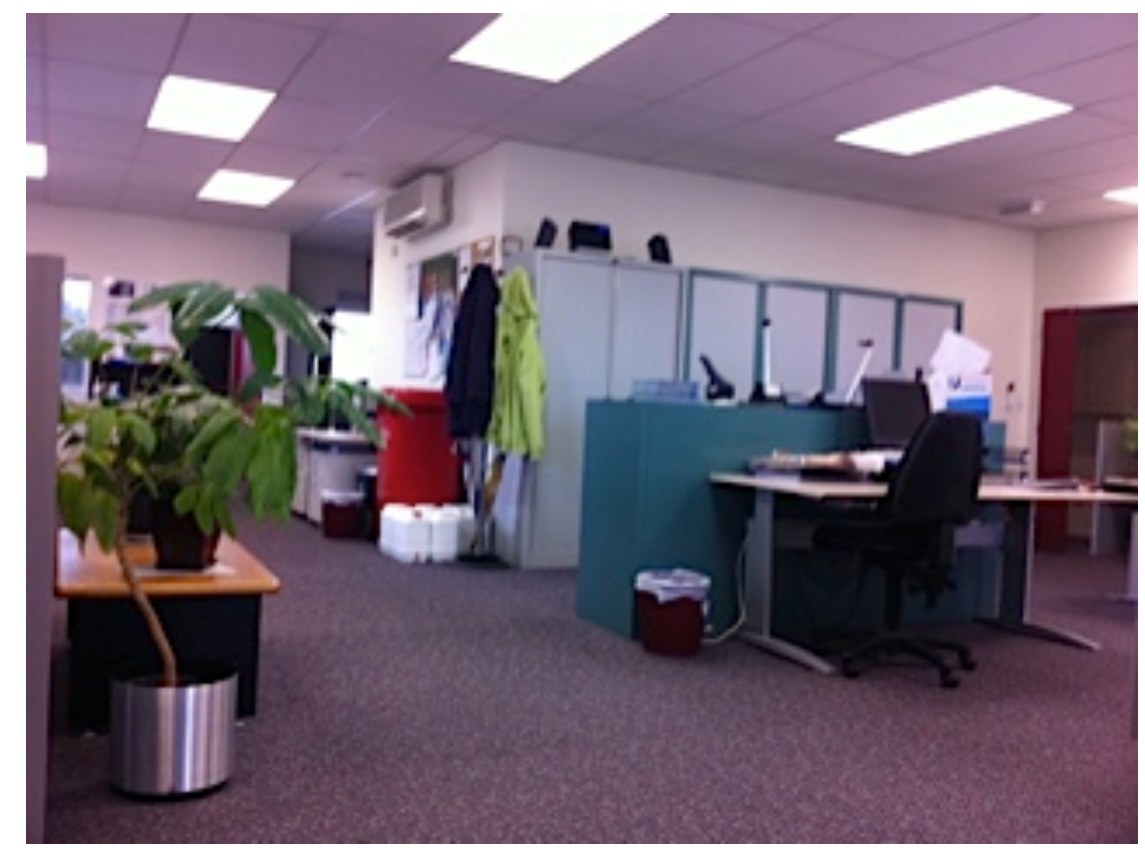

Figure 5.2: Te Puni Kōkiri work area, showing large filing cabinets

Within the regional office, the records that the team created and received were predominantly related to funds and relationships with iwi, hapū, whānau and Māori communities. Office management records were also created and received by the team, such as finance records, communications on office activities, details for Te Puni Kōkiri publications, and equipment management records.

\subsection{Recordkeeping behaviours}

This case study presents a series of vignettes to illustrate the recordkeeping behaviours demonstrated by staff within the regional office. The vignettes draw on 
data collected during the observations and interviews with the research participants as they conducted their daily work.

\subsubsection{Assist Māori to develop and implement initiatives}

One of the responsibilities of kaiwhakarite was to work with members of the Māori community to design and implement initiatives, including supporting and assisting them through the development and management of proposals for funding. As observed during the fieldwork, this involved two common behaviours, responding to queries from members of the public and using the funding management system to manage details submitted as part of a funding application.

The first vignette describes Whetu as she responds to an email query from a fund applicant early one afternoon. Whetu had been working as a kaiwhakarite in the regional office since 1997. She had a few folders on a shelf on her desks well as a filing tray on the corner of her desk. Behind the desk was a cupboard, which was closed with reference folders stacked along the top. She had two physical files on her desk, with coloured labels along the edge to indicate the specific funding applications they relate to. On this particular morning, she had been away for the morning and was returning to her desk after her lunch break. Within this vignette, Whetu responds to queries from members of the public as they develop a funding application, which involves her preparing and sending emails and describes how she uses the ICTs to perform tasks.

\section{Vignette 5.1: Responding to an email query}

Whetu is reading an email from a member of the public requesting an extension for a grant application. She clicks reply and takes some time as she composes her response. Once her response is written, she asks 
herself "Okay, have I answered everything?" as she scrolls up to the original part of the message. "Oh no, she wants copies of the application documents."

While Whetu is doing this, she is alerted to a new email message arriving by a pop-up window and a beep. She clicks on it to open the new email, reads it and clicks reply. She writes a brief response and when she sends her response, she selects the option to 'Send and Save', which opens an EDRMS screen for her to select where to save the email. After opening various folders, she clicks the button to cancel the save process and sends the email without saving it.

Whetu returns to the initial email to attach the necessary documents and murmurs to herself "oh no, where do I find these things? What am I looking for?" She returns to her desktop where she has the files saved. She opens one, "Oh, that's the wrong one." She right-clicks on another file and selects 'send to'. "Oh, that's not the way to do it." She returns to the email then back again to the desktop. She locates the correct items from her desktop and then returns to the email, clicks on the text, right clicks and selects paste, which puts a copy of the document in the email as an attachment. She completes this process separately for all four of the requested documents. Once she adds them, she clicks send and when prompted to save the message to the EDRMS, she selects 'Send without Saving'.

As described within this vignette, this staff member took a couple of attempts to complete some ICT-related processes, for example to attach documents from her desktop to an email. The researcher's presence may have influenced this behaviour; 
these behaviours may also have been influenced by Whetu's digital and recordkeeping literacy.

Te Puni Kōkiri provided training to all employees; staff members received basic training during their induction on how to use the EDRMS, and the EDRMS team based in Wellington travelled to the regional offices quarterly during the year to provide face-to-face EDRMS training. However, training on how to use standard Microsoft Office applications was not offered by the organisation and a job description for a Regional Advisor did not include reference to competencies or prerequisite skills relating to common ICTs. The organisation may have assumed that staff and job seekers were competent using Microsoft Office applications or that staff members would request technical assistance if required. Jessica explained that she had requested some specific training on Microsoft Office applications after an upgrade and had engaged with a training provider directly, approved and funded by Te Puni Kōkiri. Jessica also explained that as well as support from the organisation if they requested training, questions could also be raised with the organisational help desk; “we've also got our IT help-desk, which is an 0800 number we can always ring up."

When staff sent an email, the EDRMS automatically prompted them to save the email directly into the EDRMS, including any attachments. However, during this observation Whetu sent two emails to members of the public without saving either of them to the EDRMS; in one situation after navigating through the EDRMS folders, she cancelled the save action, possibly because she could not find an appropriate location. This behaviour suggests that while the organisation had implemented some processes to prompt staff to save email correspondence to the EDRMS, ultimately staff were responsible for deciding what records they captured in the EDRMS. Furthermore, similar to what was observed at ERMA New Zealand (Vignette 4.7), it 
was not always obvious for staff where to store documents, which resulted in records stored in information repositories external to the organisation's recordkeeping systems, in this case, the sent items folder in Outlook.

Whetu was also observed at another time working with the funding management system to retrieve and process fund-related details. Vignette 5.2 describes the process of entering application details into the funding management system from both digital and physical sources.

\section{Vignette 5.2: Entering information into the funding management system}

Whetu gathers papers about a proposal for a grant from a physical folder on her desk. She opens the funding management system. In the funding management system, Whetu enters the applicant name and application number in the search box.

The funding management system displays the details based on Whetu's search query. "Right, when was it entered?" “When did I get this?” She starts flicking through the proposal on her desk for the date submitted, eventually turning to her computer where she opens Outlook and searches for the applicant's name in her emails.

This search returns an email with the submitted application; she now returns to the funding management system and adds the details to the application profile, including the proposal details, the date received, the proposal name and a short description. She populates the Coordinator's name and she refers to a physical phone book on her desk to add in the Coordinator's phone number. The funding management system has prepopulated the Assessor field with her name. At this point, some visitors 
arrive in the office to see Whetu for a scheduled meeting, and the observation concludes.

This vignette highlights some practical ways that working in a hybrid recordkeeping environment manifested themselves at Te Puni Kōkiri. Whetu drew on details from both digital and physical records sources as she populated the applicant's profile in the funding management system; regardless of the format of the source, all of the details were manually entered into the ICTs. During the observations, records within the regional office were created and captured in both physical and digital formats, requiring kaiwhakarite and other staff to search for and to collate the various information into a single format, in this case, digitally.

This vignette emphasises the continuing role that physical records had within the daily work of the participants. While the organisation made significant investments in the ICTs infrastructure for recordkeeping and in supporting staff to use the infrastructure, funding applicants interacted with kaiwhakarite on a personal level; they knew their names, their personal interests and were invited to the office for face-to-face interactions, and submission of physical documents. This vignette also demonstrates that the agency was dependent on the efforts of staff to collate information. The functionality of the ICTs at the time of the fieldwork did not allow for the complete automation of the data collection, requiring kaiwhakarite to invest their efforts in data entry tasks. This manual approach to managing records created in both physical and digital formats allowed for the possibility of data entry errors and was time-consuming for the kaiwhakarite.

\subsubsection{Gather information to inform policy initiatives and developments}


Another responsibility of kaiwhakarite was to assist in the development of Te Puni Kōkiri policy initiatives. Vignette 5.3 describes the recordkeeping behaviours of Pamela during one observation. This participant worked with the policy team at the Te Puni Kōkiri head office in Wellington, specialising in Māori housing policy development. She travelled significantly for her role, which involved spending approximately half her working week at the Te Puni Kōkiri head office in Wellington or travelling to regional communities, including to rural and isolated areas, throughout the North Island. She did not have a permanent desk within the either Head Office or the regional office; instead, she used whatever space was available in the office or worked from home using a laptop. This vignette describes a day when she was working in the regional office, using a colleague's desk while he was not in the office. The desk that she was sitting at was clear of paperwork, although there were some personal items belonging to Pamela's colleague. Pamela was using a Te Puni Kōkiri laptop, positioned in front of the desktop computer on the desk.

\section{Vignette 5.3: Managing emails and reports}

Pamela is reviewing emails she has received on this morning. As she is doing this, the Regional Director walks past and asks “Did you get that email with those attachments - the supporting documents for that proposal? Have you done anything with them?", referring to an email sent from a member of the public.

“No, I haven't got to it yet."

"OK, I'll upload them into the EDRMS now" and he turns, returning to his office.

The next email Pamela opens is sent by the Te Puni Kōkiri communications team and contains a list of recent media articles. “Do I 
want to read it or can I catch up later?" she asks herself out loud. Pamela leaves it in her inbox and says "Now, what was I doing last night?". She explains that she couldn't sleep and got up at 2 am to review a funding proposal.

Pamela turns to pick up and open her handbag and finds a memory stick, which she connects to the laptop. Then, she navigates through the folder hierarchy (My Computer $>$ Stick $>$ MDFs $>$ Open Docs $>$ Funding Proposal) to locate the proposal she is working on, named "Application to Te Puni Kōkiri for funding v007.doc". Pamela also has a printed copy of this proposal, with some pencil edits, on the desk in front of her. She starts updating the document in Microsoft Word to reflect these pencil edits.

A pop-up message appears informing her that a new email has arrived. Pamela clicks on this to open the email message; it is the Regional Director with links to the documents that he has saved to the EDRMS. "Right, I'll delete that email now," she says as she navigates back to her inbox to find the relevant email with the attachments, which she deletes, along with the email from the Regional Director.

Vignette 5.3 describes how Pamela worked with the EDRMS and the influence of the mobile nature of her role. As she explained, her regular work meant she was often out of the office, sometimes working in remote areas without reliable Internet access; she also did not have an allocated desk in any Te Puni Kōkiri office, she had no routine travel arrangements as she could be in different parts of the country on different days and she was reliant on mobile technology, using a smartphone and laptop. During the interview with this participant, she explained how she used the 
EDRMS in conjunction with personal storage devices and how she relied on assistance from others to save documents into the EDRMS:

I have it [records] all on a stick and at the end of the year I go to the IT and say "Can you drop all that into the EDRMS please because I don't know how to?" ... I'm on the road, the connections aren't that strong and so you're trying to open a document or trying put a document in there, and it takes ages. But when I'm in the office I might do parts of it and drop them in and create the files and all that.

(Pamela, Te Puni Kōkiri)

Pamela further explained that she is often asked to review documents for colleagues, and her colleagues acknowledge challenges she has accessing the EDRMS when working remotely:

I either do track changes or comments and then I send it back to them and they'll incorporate it and then send it back as version whatever. And then they'll send me the link as to where it's saved. They've never asked me to go into the link and make the changes in there - but you can do because you can do your editing and all that. Because it's in the EDRMS and they know that I'm out and about it's easier for them to email it to me, me to make the comments on the document and they'll incorporate it.

(Pamela, Te Puni Kōkiri)

Te Puni Kōkiri granted staff discretion to manage records, including when and how staff created and captured records in the EDRMS. The EDRMS allowed staff to save records at opportunities that suited staff, not necessarily at the point that the record was created. Within this context and in light of her mobile work, Pamela devised an approach to capturing records that allowed her constant access to the records, regardless of her location. This was enabled by the organisation, as it was the IT team, which included the recordkeeping responsibility for the organisation, who saved the records on Pamela's behalf. Pamela's approach was not devised to avoid the EDRMS because she found it difficult to use or didn't have sufficient training or 
skills to use it independently: as she described "that system is quite good, because if you set them up right at the beginning with all the metadata as they call. It's quite easy to get in and out of". Pamela's approach was also not related to circumventing her recordkeeping responsibilities because during the interview with her, she explained that she understood the importance of recordkeeping within organisations as a result of her previous employment as a records clerk:

When I first started, years and years ago, at Housing Corporation it was called then, I was a records clerk. And even now I say, everyone should start in the records because you get a really good understanding of how the whole organisation operates... Really good grounding [...] And I mean, you can tell people who have never worked in records - they just don't care!

(Pamela, Te Puni Kōkiri)

This suggests that Pamela valued records as a core component of her regular work and had considered her approach to managing records she created, captured and used. None of the other Te Puni Kōkiri participants mentioned mobile work as a regular component of their roles; Pamela's requirements and circumstances were somewhat unique within the regional office.

\subsubsection{Liaise between Government and Māori organisations and communities}

A further task of kaiwhakarite was to liaise between the government and Māori organisations and communities. Vignette 5.4 describes Jessica, who worked in the regional office for over five years in an administration role, capturing details from a phone conversation with a member of the public. Jessica had three clearly labelled correspondence trays on her desk, as well as filing trays and a shelf housing a series of reference folders. The folders were labelled, for example, 'Waiata', 'Regional Stories', and 'Directorate'. On this particular morning, the office was nearly empty, 
and Jessica had spent time completing administrative tasks in preparation for the end of the month financial processing when she received a phone call from a member of the public. While Jessica was not in a kaiwhakarite role herself, this vignette describes how she captured details from the member of the public to pass on to one of the kaiwhakarite in the office.

\section{Vignette 5.4: Capturing details from a phone conversation with a} member of the public

Jessica answers a phone call. The conversation is loud enough to understand that it is a member of the public regarding the possibility of Te Puni Kōkiri providing business support for a local venture to grow vegetables. "Oh, okay, the person you need to talk to has gone for lunch. I'll get your details so she can get back to you."

Jessica takes a Client Enquiry form from the filing tray on the shelf on her desk and asks the caller for the details to complete the form starting at the top of the one-page form. "Right, so how would you summarise your query? ... Can you please give me your contact details - I need a phone number and a physical address? ... What's your current employment? ... Um, it's not hydro-related is it? That's one of the questions on the form... Is this to strengthen an existing business or regarding a new business? And last one, can you tell me how you heard about this service?"

“OK, thank you. I'll pass these details on to my colleague when she's back from lunch". With the physical form completed, Jessica walks to her colleague's desk where she leaves the completed form. 
During another observation, Jessica facilitated the capture of details from a member of the public, this time where the member of the public visited the office.

\section{Vignette 5.5: Capturing details from a member of the public's visit}

A member of the public comes up the stairs and stops at Jessica's desk, which is the first desk immediately upon entering the office. She has some reports to submit as part of an application and would like to talk to a particular staff member.

“She's not here right now, but let me just call her and see how far away she is" Jessica suggests. However, she gets no response when she tries to telephone her.

So, the visitor provides the reports to Jessica to pass on. Both of the reports are printed in colour, bound and in plastic cover sheets. Jessica stamps today's date on both. On one of these, she puts the stamp on the back of the last page as the first page has a lot of content on it and she did not want to cover any of it. Jessica writes brief details about the visit on a piece of sticky notepaper, which she attaches to the top report and assures the visitor she will pass them on. When the visitor leaves, Jessica walks to her colleague's desk, where she leaves the two reports.

These two vignettes highlight that although email and online interactions were common and provided important ways for members of the public to interact with kaiwhakarite, traditional methods of communication, including telephone and faceto-face interactions, remained common methods for members of the public to interact with this regional office. While unsurprising that multiple and ICT-enabled 
methods of communication provided significant ways for government and Māori organisations and communities to communicate, from an organisational perspective it required that both digital and physical recordkeeping systems be maintained and embedded within the organisation, and for both to be used and accessible by staff. This vignette illustrates how the transition to digital government was an ongoing and complex change that was dependent on many factors, including the behaviours of public servants, as well as the behaviours of the members of the public liaising with government agencies.

These two vignettes also reflect the important relationship that kaiwhakarite had with members of the public. While these two interactions involved the sharing of some personal details, in particular contact details of individuals, the nature of information contained within the funding applications could also likely contain records considered taonga ${ }^{9}$ by iwi, hapū, whānau and Māori communities requiring sensitive handling and management. The observations at Te Puni Kōkiri also reflect a unique relationship between members of the public and front-line public servants, where citizens often came to the office for informal visits, reinforcing the personal nature of the kaiwhakarite role as having direct contact with Māori people.

There was limited evidence of cross-agency information sharing during the fieldwork at Te Puni Kōkiri, although Whetu described a situation where nongovernment organisations within the region requested contact details of marae ${ }^{10}$ from the regional office:

\footnotetext{
${ }^{9}$ Taonga is defined as "treasure, anything prized - applied to anything considered to be of value including socially or culturally valuable objects, resources, phenomenon, ideas and techniques" by the Māori Dictionary (online) at www.maoridictionary.co.nz (accessed 1705 2017).

${ }^{10}$ Marea is defined as "the open area in front of the wharenui, where formal greetings and discussions take place. Often also used to include the complex of buildings around the marae" by the Māori Dictionary (online) at www.maoridictionary.co.nz (accessed 1504 2017).
} 
Some of the work we've done has been with marae and so they give us all their contact details and that's all they do, oh plus they answer specific questions for us. And we receive requests from the large [regional] entities for those details. Because they need to make contact with the marae and why not use what the crown has collected. So, um... after much, much to-ing and fro-ing our Regional Director decided that we can pass that information on, just their contact details, that's all. We can pass that on because it's all for the benefit of those people, that's how we look at it. (Whetu, Te Puni Kōkiri)

This explanation from Whetu demonstrates the unique position that the regional office holds within the broader region and the position of trust that both citizens on behalf of marae, and other organisations place it in. While there are specific requirements defined within the Privacy Act that control how details collected by one agency is shared with other organisations, the focus of this regional office and of the kaiwhakarite was on providing benefits to the Māori people of the region.

\subsection{Discussion}

This section explores two themes identified based on the fieldwork: that staff followed individual approaches to using the recordkeeping systems available, and that recordkeeping was partly ICT-enabled.

\subsubsection{Personal approaches to recordkeeping}

During the fieldwork, the participants demonstrated personal ways of working with public records that they created or received as part of their jobs. Each of the participants employed different recordkeeping behaviours based on various factors, including their roles and professions, work practices as well as technical skill levels. While the EDRMS was implemented to manage all digital business-related documents, the same recordkeeping requirements were set for all staff by the relevant organisational policies and all staff received the same training and support, 
participants had discretion to apply personal approaches to creating, managing and using records.

Staff decided what records to capture. For example, Jessica routinely saved emails in the EDRMS that appeared administrative, such as catering requests and booking confirmations, whereas Whetu was observed not saving emails into the EDRMS that included discussions with members of the public concerning details of their funding applications. Participants mentioned the need to create and capture records to provide evidence that a specific activity or interaction had occurred, and to provide a history of interactions between Te Puni Kōkiri and various entities over long periods of time, which they occasionally referred to:

Housing on Māori land anyway, it's quite a complex process and so I sort of refer back to where I was way back then and have we broken down barriers and how long has it taken? What was it like back then? What is it like now?

(Pamela, Te Puni Kōkiri)

Within the organisational context, staff also made decisions about where to capture records. Staff used a variety of ICTs, in addition to the EDRMS; Whetu was observed storing draft application documents on her personal desktop, and Pamela stored proposals and policy records on personal storage devices until the IT team imported the documents into the EDRMS on her behalf. While all participants were observed saving final versions of items to the EDRMS, similar to the ERMA New Zealand participants, Te Puni Kōkiri participants mentioned their concerns about when colleagues had access or visibility to the records and how they could retain control over this. 
Participants explained that if they considered the record 'important enough' then they would save it in the EDRMS, although there was no agreed definition or approach to defining the important records:

If it's a decision I suppose, that this is going to happen, we've agreed to this, then I'll keep it. But if it's just a to-ing and fro-ing about getting to a point, I don't keep it. (Pamela, Te Puni Kōkiri)

Similar to ERMA New Zealand, there was no regular or routine monitoring by the organisation of what records were captured by staff and what system they were consequently captured in.

The fieldwork also indicated that some staff resisted using the EDRMS. One participant, Jessica, suggested that some staff in the office had found the transition to ICT-enabled recordkeeping, and in particular the use of the EDRMS, difficult. She explained that it had been a significant change in mindset for staff, some of whom showed their displeasure by resisting using the EDRMS. She admitted that while it took her some time to become familiar with it, she is now an office EDRMS 'champion':

Yeah, it's a challenge for me, to try to lay down all the benefits of it, all the pros, but they just don't want to change, or they try... some are good.

(Jessica, Te Puni Kōkiri)

While the ICTs used by Te Puni Kōkiri staff to manage records included some mechanisms to encourage and remind staff to save records as part of the business process, the organisation allowed staff to bypass these; for example when Whetu selected the option to "Send without Saving" when she sent an email to funding applicants. As Whetu explained, this allowed staff to save the final email conversation, containing the complete conversation as one item, rather than as 
separate items in the EDRMS, thus improving search and retrieval of these items, as a complete conversation, in the future.

This varied use of the EDRMS and approaches to recordkeeping by the participants is interesting, particularly considering the extensive technical infrastructure and organisational framework for recordkeeping established to manage records within Te Puni Kōkiri, including policies, training, records support staff, physical filing infrastructure for both head office and regional offices, and the EDRMS. This approach to recordkeeping aligns with that recounted by White $(2007$, p. 230) who described a personal approach to recordkeeping within her analysis of the New Zealand Official Information Act, suggesting that public servants made judgments every day about what is worth keeping either for their immediate use or the longerterm departmental record. White (2007, p. 258) argued that an ad hoc and individual approach to recordkeeping is prevalent within the New Zealand public sector, beyond any single agency: public servants constantly delete trivial emails, throw incidental notes in the bin and make choices about which changes to a document they should save as a new draft and which can simply override the previous versions.

\subsubsection{An evolving ICT-enabled work environment}

This case study suggests that the ways public servants work are changing as a result of increasingly ICT-enabled processes. This situation is not unique to Te Puni Kōkiri; however, the empirical fieldwork highlights some of the more practical recordkeeping challenges faced by the participants.

During the observations, participants often used multiple information sources to collate details required to complete business tasks. For example, in Vignette 5.2, Whetu worked between digital records and physical records in files. When entering 
an applicant's details into the funding management system, she collated details from the physical papers in front of her, from emails stored in Outlook, from digital files stored in the EDRMS and checked a phone number in a physical phone book from her desk. While this process involved manual steps for Whetu to collate the relevant details together in the funding management system, this degree of integration from such disparate sources would have been far more time-consuming and complex in a purely physical environment. The recordkeeping systems in this regional office, were predominantly ICT-enabled including the use of the EDRMS, the funding management system and Outlook; however, paper remained a significant format to create, manage and use records. The importance of paper as a recordkeeping format was particularly relevant when dealing with members of the public; for example, when a member of the public visited Jessica at the Te Puni Kōkiri regional office to submit physical reports as part of an application.

While traditional communication channels remained important for relationship management between Te Puni Kōkiri staff and iwi, hapū, whānau as well as Māori communities, during the observations and interviews, participants made statements where one could conclude that their preference was for records to be created and managed in digital formats. For example, Jessica explained that if staff received business cards, they transposed the details into the shared digital address book and disposed of the physical business card. This digital address book was then shared amongst the team and contained details on over one thousand contacts. Previously, contact details had been managed in each staff member's physical personal information management environment.

Evidence of this evolving ICT-enabled work environment is also provided in the Te Puni Kōkiri Retention and Disposal schedule, approved by Archives New Zealand in 2016. This report quantified the digital and physical records managed by Te Puni 
Kōkiri, with significant numbers of records existing in both formats. In 2016, there were approximately two million born-digital and digitised records saved in the EDRMS, with approximately 175,000 records added each year (Te Puni Kōkiri, 2016, p. 4). At the same time, approximately 835 linear metres of physical records were held onsite at the Te Puni Kōkiri head office in Wellington, with approximately 1,245 linear metres of physical records held offsite at a specialist records storage company (Te Puni Kōkiri, 2016, p. 5).

The use of increasingly ICT-enabled processes and digital records assumes that staff have a basic level of technical skills. While training was provided to staff when Te Puni Kōkiri introduced any new systems, and system-specific training was provided every quarter at the regional offices throughout the year, the agency assumed, at least to some extent, that staff had necessary prerequisite ICTs skills. None of the three agencies involved in this research, including Te Puni Kōkiri provided staff with training on using common ICTs or on basic, prerequisite ICTs skills. This approach can be challenging with an ageing workforce and team, and was observed in Vignette 5.1 as Whetu struggled to complete a routine task using the available ICTs.

The training provided to Te Puni Kōkiri staff as portrayed by the participants, also appeared to be focused on the 'how to' components of recordkeeping, for example, how to save a document or how to apply metadata. Less emphasis was placed by the participants on the broader elements of recordkeeping, for example the organisational benefits, why metadata was important or their specific recordkeeping obligations according to Te Puni Kōkiri policies. 


\section{Recordkeeping behaviours at Inland Revenue}

This chapter describes the third and final case study conducted at a regional office of Inland Revenue between August 2014 and December 2014. This chapter introduces the agency's purpose, its primary functions and the recordkeeping environment, and describes the three Inland Revenue organisational units involved in the fieldwork. The chapter concludes by describing the participants' recordkeeping behaviours.

\subsection{Inland Revenue}

Inland Revenue is New Zealand's principal revenue agency established under the Inland Revenue Department Act 1974 and following the repeal of that Act, the Tax Administration Act 1994 (TAA), which provided for the continuation of Inland Revenue. Inland Revenue has two main functions; the first is to collect core Crown revenue as New Zealand's principal revenue agency. In 2013-14 Inland Revenue received $\$ 56.2$ billion in tax revenue as well as $\$ 1.1$ billion in other income (Inland Revenue, 2014b, p. 10). As part of this, Inland Revenue received and managed over one million individual annual tax returns, two million employer monthly schedules, over 380,000 company returns, and three million GST returns (Inland Revenue, $2014 b$, p. 10). The second primary function of Inland Revenue is to administer social policy programmes often conducted in conjunction with other agencies, including Working for Families tax credits, child support, KiwiSaver, student loans and paid parental leave (Inland Revenue, 2014b, p. 51).

A significant component of both of these core functions is engaging and communicating with members of the public. In 2013-2014 Inland Revenue facilitated 5.53 million customer service interactions, including customer enquiries on tax and social support programmes through digital channels, paper correspondence, telephone, personal appointments, community focused communication approaches 
including seminars as well as through the complaints management service (Inland Revenue, 2014b, p. 51).

In 2014, at the time the fieldwork was conducted, Inland Revenue was the third largest New Zealand government department (State Services Commission, 2014). At 30 June 2014, Inland Revenue employed 5,641 full-time equivalents, structured into the following functional areas across 17 locations:

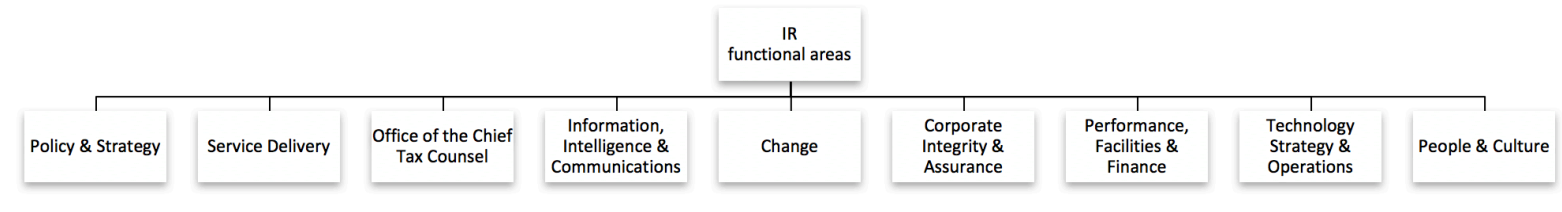

Figure 6.1: Inland Revenue's functional areas (Inland Revenue, 2014c, p. 9)

\subsubsection{Information management at Inland Revenue}

As well as complying with the legislative framework for recordkeeping, Inland Revenue had specific responsibilities relating to the collection of information under other legislation, including the TAA. Under the TAA, the powers of the Inland Revenue Commissioner and, by delegation, other Inland Revenue staff were extensive. For example, Section 17 of the TAA empowered the Commissioner to require any person to provide in writing any information and to produce for inspection any documents that are considered necessary or relevant to exercise the Commissioner's statutory functions (Inland Revenue, 2013). Section 17 of the TAA, which relates to requisitions for information, was one of Inland Revenue's primary information-gathering methods. Some holders of information, such as banks, required Inland Revenue to state its legal authority before they would release the information. Where Inland Revenue required information from persons other than 
the taxpayer and cooperation was likely, Inland Revenue would initially seek the information by a letter, which referred to obligations under Section 17 (Inland Revenue, 2013). Section 157 of the TAA concerns the use of statutory notices that Inland Revenue issue to banks requiring them to make deductions from their customers' accounts (Inland Revenue, 2011).

While Section 17 related to information collection powers, the TAA also allowed for extended retention periods of tax information. Section 163 of the TAA, stated that there is "No limitation of action to recover tax, including that no statute of limitations shall bar or affect any action or remedy for the recovery of tax" (Tax Administration Act 1994, 1994). The impact of this section was that Inland Revenue could retain all records relating to tax collection for as long as it considered the records had business value and for as long as those records might facilitate the ability for Inland Revenue to collect revenue, overriding any retention and disposal requirements set within Archives New Zealand schedules (Inland Revenue, 2009a, p. 2).

Adding to the complexity of the legislative environment at Inland Revenue, were the secrecy provisions of the TAA and the Child Support Act:

An important way in which the tax system's integrity is protected is by Inland Revenue not disclosing tax secret information it has received about a taxpayer to someone else. There are criminal sanctions that can be imposed if an Inland Revenue employee fails to comply with those secrecy obligations (Inland Revenue, 2012).

The exchange of certain information between Inland Revenue and other government entities was limited to specified purposes (CCH New Zealand Limited, 2013, pp. 6365). Section 81(4) of the TAA contained some exceptions that specifically allowed Inland Revenue to disclose certain information (Inland Revenue, 2012). The Privacy 
Act defined approved information sharing agreements between Inland Revenue and other New Zealand agencies (Privacy Act, 1993).

The relationship between the Privacy Act, the Official Information Act and the Tax Administration Act was complex. If an information request was made by or on behalf of a person for information about themselves, Inland Revenue considered it according to the Privacy Act. Other requests, including requests for information relating to someone else or requests from corporate organisations for information, are considered under the Official Information Act (Inland Revenue, 2012). The provisions of the Official Information Act and Privacy Act did not derogate from the secrecy provisions contained in the TAA. If the TAA precluded the disclosure, the reason for the refusal has to be one of the reasons stipulated based on specified sections of the Official Information Act or the Privacy Act (Inland Revenue, 2012).

The information management and recordkeeping requirements were communicated to staff primarily through the Code of Conduct and ongoing training for all staff. At the start of a staff member's employment within Inland Revenue, they were required to complete online Code of Conduct training and there were regular reminders throughout the year regarding staff obligations set within the Code of Conduct:

\footnotetext{
Refreshers, umm, a Code of Conduct thing that we do every couple of months in our team meetings, we choose a topic and just cover it to make sure everyone's on the same page and they have to sign off doing that. So it's really hammered into you, which is good because it needs to be. (Courtney, Inland Revenue)
}

In addition to the Code of Conduct, there were comprehensive policies and procedural guidelines for staff to assist in determining what information could be released to whom, at which point in time and what information was required to be captured: 
So we've got policies that say this is what can be released and then there's also, if you need to do anything, there are specific instructions and it's a literal step-by-step instruction for everything.

(Tracy, Inland Revenue)

\subsubsection{Information technology at Inland Revenue}

These recordkeeping requirements existed during a period of significant technological change for Inland Revenue. The Business Transformation Programme was a major component of this technological change. The aim of this programme was to modernise the revenue system through business process re-engineering (Inland Revenue, 2014a, p. 42). Approved by Cabinet in November 2014, the programme commenced in early in 2015 and, at that time, was expected to take ten years to complete, to be delivered in four stages.

One of the main focuses of the Business Transformation Programme was IR's core operating system, Future Inland Revenue Systems and Technology (FIRST). Primarily, FIRST was a revenue collection system, designed to support the administration of the tax regime. Implemented at Inland Revenue in 1991, FIRST identified and registered customers by number, calculated tax liabilities and social assistance entitlements and obligations, amounts owing or refunds due, handled returns, correspondence and recorded tax totals for Crown revenue purposes. As Inland Revenue described to the incoming minister in 2014, FIRST was limited in the current tax administration environment:

Using it [FIRST] to administer social assistance programmes such as Working for Families tax credits results in both policy and administrative tradeoffs being made to ensure that the policy and administrative framework can be accommodated. Furthermore, FIRST was designed for a paper-based system where information from the taxpayer's paper tax return was entered into the system with the output generated in paper form and sent to the customer. It therefore does not cope well with demands 
for online access and immediate responses expected by today's customers. This increases both compliance costs and administration costs (Inland Revenue, 2014a, p. $42)$.

Each entry in FIRST was automatically assigned a unique Document Lodgement Number (DLN). This 14-character number was used as the reference number for each information asset sent externally from Inland Revenue and provided the unique reference number for physical records.

Front-line staff regularly used an extensive array of ICTs in addition to FIRST, each of which had differing degrees of integration with FIRST (refer to Table 6.1 for details on the ICTs used by participants during the observations).

Table 6.1: Inland Revenue ICTs used by participants

\begin{tabular}{|l|l|}
\hline \multicolumn{1}{|c|}{ ICTs } & \multicolumn{1}{c|}{ Description } \\
\hline Data Warehouse & $\begin{array}{l}\text { Provided a search interface to a large data warehouse of customer } \\
\text { details. Once users performed the search, the system presented the data } \\
\text { in table form. }\end{array}$ \\
\hline EDSR & $\begin{array}{l}\text { Electronic Data Storage \& Retrieval system, which stored scanned } \\
\text { images of returns, cheque and remittance images and statements and } \\
\text { notices issued to customers. }\end{array}$ \\
\hline Express Client & A phone system, used by staff to make and receive calls. \\
\hline Knowledge Base & $\begin{array}{l}\text { A web-based repository of policies, procedures and supporting } \\
\text { materials designed to provide answers to employees' common } \\
\text { questions. }\end{array}$ \\
\hline $\begin{array}{l}\text { LOLA - Letters } \\
\text { online }\end{array}$ & $\begin{array}{l}\text { Generated letters based on templates. Staff populated details in the } \\
\text { template before LOLA automatically prepared the letter for } \\
\text { distribution, either digitally or physically for posting. }\end{array}$ \\
\hline eCase & $\begin{array}{l}\text { A case management solution that included the following functionality: } \\
\text { document management } \\
\text { search } \\
\text { diary management } \\
\text { targeted reporting } \\
\text { workflow management }\end{array}$ \\
\hline
\end{tabular}


SVOC

A phone system, which staff logged into to make and receive calls. This system also calculated statistics about the phone calls, including average handling time, talk times, and break times.

Within this legislative and ICTs environment, recordkeeping approaches were decentralised within the agency, with each business unit responsible for its recordkeeping. In the 2009 Appraisal Report submitted to Archives New Zealand, Inland Revenue described the recordkeeping environment as:

Currently, Inland Revenue has no centralised control over recordkeeping systems, practices or personnel. Each business unit is responsible for its own administrative and business records. A corporate unit within Head Office, however, is responsible for developing a wide range of standards, assisting with implementation, providing advice and, increasingly, monitoring compliance (Inland Revenue, 2009b, p. 5).

\subsubsection{The three units involved in the case study}

The case study investigation conducted at Inland Revenue involved three units, all situated within the broader Service Delivery functional area. The Service Delivery function provided information and services directly to IR's customers to help them meet their tax obligations and manage their social entitlements ( $\mathrm{CCH}$ New Zealand Limited, 2013). As a department, Service Delivery provided support to taxpayers using a multitude of channels, including face-to-face, phone, correspondence and online channels. Service Delivery also developed new customer products, managed investigations, returns and arrears, managed IR's litigation and was the main point of interactions for customers making payments and filing returns.

The three units involved in developing the Inland Revenue case study were Child Support, Debt 2010 and Community Compliance. Child Support's core business was to assess, collect and disburse payments from parents who no longer live with their 
children, to help financially support those children. The second unit involved in the case study was Debt 2010. This unit managed a ten-year debt collection programme within Service Delivery. The programme had between 170-200 Collection Officers, Customer Service specialists and support staff operating and supporting the programme. The third unit involved in the case study was Community Compliance. The role of the Community Compliance unit was to assist members of the public and businesses in meeting their obligations by providing information, reminders, selfassessment tools and online services (Inland Revenue, 2014d).

Each unit created, captured and managed different types of records; the following sections describe in more detail specific types of records managed by each unit. However, there were some categories of records consistently managed by the three units: taxpayer records in FIRST, which contained personal details; interaction history between the clients and Inland Revenue staff; copies of legislation, legal opinions and guidelines or references for specific applications of the legislation; as well as time and activity monitoring records. In particular, the taxpayer records were highly sensitive.

\subsection{Recordkeeping behaviours}

This case study focuses on recordkeeping behaviours demonstrated by the participants as they went about their daily work, representing the three business areas involved in the research: Child Support, Debt 2010, and Community Compliance.

\subsubsection{Child Support}

The Child Support unit operated according to the requirements of the Child Support Act 1991, one of the Acts administered by Inland Revenue. Inland Revenue accepted applications for child support from custodians, calculated how much support a 
liable parent was required to pay and collected the amount due (CCH New Zealand Limited, 2013, p. 588). Inland Revenue also collected and paid domestic maintenance, court-ordered child maintenance, and any maintenance payable under a voluntary agreement (CCH New Zealand Limited, 2013, p. 588).

The Child Support unit operated based on the following standard process:

1. The person caring for the child, the custodian, applied for child support, which involved completing a form and sending the signed copy of the completed form along with proof of the date of birth for each child to Inland Revenue.

2. Inland Revenue applied a standard formula to calculate how much child support must be paid by the paying parent, the parent who does not care for the child on an ongoing basis. The standard formula calculated the paying parent's taxable income, removed a standard living allowance, depending on their living arrangements, and multiplied the result by a percentage based on the number of children.

3. Inland Revenue divided the annual calculated amount into monthly amounts and informed the paying parent how much they needed to pay. Inland Revenue then notified the custodians of the child support payments they would receive.

4. Child Support collected payments from the paying parent and passed them on to the custodian to assist with the care of the child or the government if the custodian was receiving a sole parent benefit. 
From a recordkeeping perspective, Child Support officers created, managed and used details about the custodian and paying parents, such as name, address, date of birth, details of the children as well as income and spending details using the FIRST system. Child Support officers also captured details about each interaction with all parties in FIRST, so that Inland Revenue had evidence of these interactions.

\subsubsection{Notify custodians of child support payments}

This vignette describes an observation of a child support officer, Deanna, who had been in her role at Inland Revenue for less than six months and had previously worked in call centre roles in the private sector. At the time of the observation, Deanna was coming to the end of a morning two-hour phone shift. During her phone shift, Deanna used a spreadsheet saved on her desktop to monitor her work performance, including noting details about the customer, the call start and finish times, and any follow-up tasks she had to compete as a result of the conversation.

While Deanna's phone shift for this particular morning had not yet finished, she had received six phone calls, and according to the spreadsheet, her average call time was just over 12 minutes. This observation concerns interactions between Deanna and a custodial parent where there were changes occurring in benefit payments, and the custodial parent queried specific timeframes for the changes. Deanna worked with this customer to determine the child support payments and the timeframes for them; this corresponds to the third stage of the child support operating process, where Inland Revenue established the payment arrangements.

\section{Vignette 6.1: Responding to a query from a custodial parent}

Deanna is on the phone asking some specific questions to the customer about their situation and adding some brief notes to the spreadsheet that she has open on one of her monitors. When the call is complete, she 
clicks the 'Wrap Up Call' SVOC button open on her other monitor and then logs off the phone system, indicating the end of her phone shift.

She opens a FIRST screen and starts updating the details for this call, including adding a reference to another Document Lodgement Number (DLN). To do this, she has the two FIRST screens open on one monitor and copies and pastes the DLN number between the two.

She opens the Inland Revenue Knowledge Base on the Intranet; she then navigates to a particular page, which outlines a series of steps relating to changes in benefit payments and she starts reading each step. She also gets a little notebook from her shelf and opens this up to a particular page; it appears to be handwritten personal reference notes.

After adding some details in the FIRST screen, she turns around to tap her colleague on the shoulder, asking him to verify the update she has just made. She gives him a Post-it note with the DLN number on it. He takes this back to his desk, and after a brief time he returns the Post-it note and says to Deanna "all done". "OK I'm going to ring her back - I said I'd ring her back in a couple of days but I've worked it out, so I'll let her know now."

After a brief discussion, Deanna makes a note in her spreadsheet about a compliment from the customer that she is happy with the service. She updates a piece of paper sitting on her desk shelf that she uses to monitor her activities and time throughout the day; "that follow-up took about 20 minutes". 
This vignette provides some insight into the nature of the Child Support work; Child Support Officers follow prescribed instructions to complete their work activities. The combination of prescriptive work processes and dependence on ICTs to complete the work is evident from this observation, although it is also interesting to note that some of the prescribed work processes were not initiated or embedded within the ICTs. Deanna's colleague explained that because Deanna's updates involved benefit payments, he was required to perform a 'double check' on details entered by Deanna; "I'm checking that the details, the names, amounts, dates and codes are all correct". He further explained that there is a similar process for entering bank account details, but only two people in their team could verify bank account details, "this is to stop you putting your own number in". While important to ensure that these types of work processes are completed, the FIRST system did not prompt Deanna or initiate a process to ensure the check occurred; Deanna initiated the process for her colleague to review the changes in benefit payments for a custodial parent.

\subsubsection{Establish child support payment arrangements}

Vignette 6.2 also describes this prescriptive working environment. Vignette 6.2 describes Laura, who is another Child Support officer who worked on a different floor in a different Child Support team to Deanna. Laura worked in an open-plan environment, in a line of three desks with a further three desks directly opposite. The desks are positioned at the far end of the open-plan floor so there was a wall directly behind her, covered in colourful motivational posters. Laura has worked in the team for one year.

This vignette describes Laura, after a phone call with the custodian, as she updates client details where a shared care arrangement has ceased. This process involves updating FIRST and using LOLA to generate a letter to the custodian. 


\section{Vignette 6.2: Updating customer details}

Laura has two FIRST screens open. She copies text from a word document and pastes it into a FIRST screen and continues updating details in FIRST.

She opens and logs into LOLA. She selects a letter template from a dropdown list of templates she has recently used, which opens a pop-up window where she adds the customer's IRD number. This step opens a new screen displaying the new letter, addressed to the custodian; there are some radio buttons and text boxes on the left-hand side that Laura needs to complete. She opens a calculators page in the Inland Revenue Knowledge Base and opens the 'Count Days From' calculator. Laura returns to LOLA to add the resulting number to the letter before continuing to update further details on the left-hand of the screen. She clicks the 'Next' button; but is unable to continue as she missed one radio button, which is highlighted red. She completes the text required and selects the 'Next' button.

She then returns to updates the FIRST screen, adding further details: "A/N has confirmed that both children left her care as of [date]. Children are now in the care of their father. Child support ceased for the child, [name], at an earlier date of [date]."

She checks another FIRST screen to confirm the date, before returning to the letter in the LOLA system. She clicks some buttons a few times before creating a second letter; this one addressed to the paying parent. She adds some details, reviews it on screen and then clicks publish, before making some more updates in FIRST. 
Vignettes 6.1 and 6.2 illustrate Child Support officers using a range of different Inland Revenue ICTs to complete prescribed tasks. Use of these ICTs, with a high degree of familiarity, was critical as the officers required instant access to correct details in response to questions from members of the public. In addition, both officers used non-technical solutions to create and manage ephemeral documents during this observation, including using a Post-it note to communicate details to a colleague, a notebook with reference notes, and using pieces of paper to record time spent on work activities to be entered into the time recording system later in the day. While the use of ICTs to complete the Child Support work as observed was critical, the use of traditional physical records remained important, particularly for ephemeral, supportive details, specific to individual officers, with short-term value.

Deanna described the Child Support working environment as working with '12 lines of string'. Deanna described this situation based on a thirty minute period, "I just finished one person with those benefit payments. I started doing report work about an employer not making a child support payment. And I got a call about Shared Care work that I've been looking at." This '12 lines of string' metaphor is also reflective of the recordkeeping environment within Child Support, where staff created, managed and used various records, using both ICTs and non-technical systems, within the highly complex legislative environment.

\subsubsection{Debt 2010}

Debt 2010 is a ten-year debt collection programme operating as a unit within the Service Delivery function. One of the participants involved in this research, Courtney, described her role as a Collections Officer: 
It's basically recovery and enforcement... Me, personally I work a lot with the Campaign Coordinator, we work a lot with our Team Leader, our Area Manager and general public... sometimes Legal Technical Services, which is LTS. And apart from that, we have internal contacts if we have issues with anything that we come across. We do also have contact I suppose when we're trying to get further information, so anyone you can think of... like Births, Deaths and Marriages, banks, everything like that.

(Courtney, Inland Revenue)

The Debt 2010 vision of debt management encourages taxpayers to file tax returns and make tax payments on time, with multiple and varied interventions for those who do not comply (Organisation for Economic Co-operation and Development, 2014, p. 35). Some of the interventions employed by the programme include preventative messages, 'lighter touch' assistance for customers to achieve compliance, 'Just Pay' now letters, 40 to 75-day letters, text messages and deduction notices (Organisation for Economic Co-operation and Development, 2014, p. 35).

During the observations with participants from the Debt 2010 unit, significant amounts of time were spent on the phone with customers discussing their situations, making arrangements for repayments of significant debt owed to Inland Revenue, as well as preparing and researching customers' situations based on the details stored in the FIRST system.

\subsubsection{Research customer's tax and debt situations}

This vignette describes time spent observing Courtney while she was on a phone shift from $12.45 \mathrm{pm}$ to $4.30 \mathrm{pm}$. While Courtney was on the phone shift, unlike during the observations with Child Support officers, the calls were not continuous, allowing her opportunities to complete other tasks in-between calls. During this vignette, Courtney was drawing on sections of the TAA to request information from banks and Immigration New Zealand, about specific members of the public. 


\section{Vignette 6.3: Requesting information from organisations}

Courtney is updating a FIRST screen, which is open on one of her two monitors. She has Express Client open on the other monitor, the system used to make and receive phone calls. The status bar on Express Client is green, indicating she is available to answer calls.

There is a customer completed physical form in front of her - 'Notice to Deduct Amount Owed to Inland Revenue', which has some handwritten pen markings on it. From a letter tray on a shelf on her desk, she takes a page of pink barcode stickers, each reflecting a unique DLN number, and adds one sticker to the bottom of the first page of the physical form. She returns to FIRST to enter the details of the DLN in FIRST, which involves entering the number from the barcode into a FIRST screen and a description of the physical form. Once she has completed this, she moves the physical form to the side of her desk, ready to be put in the internal mail to send to the Processing Centre for filing.

Courtney brings up Outlook, where she has a recently received email from BNZ regarding a customer's name and IRD number that do not match. She returns to FIRST and navigates some windows to a page where she enters details that the Section 157 failed. Courtney also adds notes about sending a Section 17 request to Immigration as she cannot find an income source for this customer. She returns to Outlook and creates a new email message: "Could I please have an Immigration search on the following individual?" and she provides their name, IRD number, and date of birth. She sends this email to an Immigration New Zealand email address, rather than to a named individual.

She leans over and updates a little piece of paper on her desk to the side 
of her keyboard, which has tally marks representing work activities she has completed so far during the day.

Courtney returns to Outlook, where her inbox is displaying a collection of read emails with follow-up flags. She opens one of these, reads it and then opens the LOLA system and selects a letter template to request information from a bank. The new letter opens ready for Courtney to complete the details. FIRST is open on the other monitor, and she navigates through some screens before beginning to copy and paste the customer's details into the LOLA letter. Once she completes the necessary details, she selects the option to 'Publish', which finalised the letter and forwards it to another team to mail to the bank.

Courtney requested information from other organisations and government agencies, employing Section 17 of the TAA multiple times during the observations with her. Rachel K explained the process of using Section 17 of the TAA to request information relating to an individual:

It's a formal letter and it goes to a, like a central point that we've got a contact person, like it's all formal, so for each say government department or whatever, you'll have a contact person and an address as to where you have to send these to, and they all have to go through this central point of contact. And the letters are all recorded in our system [...] The letters are generated through LOLA but you can't view them through LOLA. You have to view them through EDSR. [...] You have to put in the letter the IRD number [and] as much identifying information as you can, so they can give you the information that you're requesting [...] And they have to comply with a Section 17 notice.

(Rachel K, Inland Revenue) 
This vignette also described Courtney preparing a physical record for filing. Courtney explained that the Debt 2010 officers received mostly physical records from external organisations in response to information requests. Inland Revenue manages the physical files in Processing Centres based in Wellington, Hamilton and Christchurch; staff sent physical records required for filing to the Processing Centres and staff could also request access to the files from the centres using the DLN.

The process that Courtney followed, and as described by Karen and Deanna, indicate that there was a clear delineation between the responsibilities of staff who received the physical records and the physical act of filing the physical records as well as any on-going management of that paper, such as retention and disposal or scanning of the physical records.

\subsubsection{Make arrangements for repayments of significant debt owed to Inland Revenue}

Vignette 6.4 describes a Collections Officer, Brenda, as she works on different tasks at the start of her afternoon phone shift. Brenda worked on the same team as Courtney, sitting on the same floor but not directly near her. She had been in the role for less than a year, but employed by Inland Revenue for over thirteen years, previously working in a team dealing with student loans. This observation describes Brenda, as she reviews the financial transactions of a customer who is in debt and may meet the hardship criteria set by Inland Revenue, while on a phone shift, waiting for calls from customers.

\section{Vignette 6.4: Reviewing a customer's situation}

Brenda has a Disclosure of Financial Statements calculator open on one monitor and she is populating this with details from a physical application form, laid out in front of her. There are several printed bank 
statements stapled to this physical application form.

Brenda prints the Disclosure of Financial Statement, which includes the details she has just entered, and locks the PC as she goes to the printer, which is two desks away. As she returns seconds later, she says, "I'm paper-based, if I don't have something to put notes on, I'm shocking".

Brenda chats to her colleague in the desk across from her, asking about the recent phone activity. They discuss how they have not been very busy; he has received six calls in the previous three hours.

She opens SVOC and selects the option that 'Agent Status' is 'Available', indicating she is available to receive calls from members of the public. She then brings up FIRST where she returns to reviewing details concerning this particular customer; "what's his debt?". "Ahh", she says out loud after reviewing several FIRST screens, "he got paid Working for Families from Work and Income and from IRD for one year, that's how he's in debt". She continues reviewing the notes in FIRST, including his partner's details. She makes some notes in pencil on the Disclosure of Financial Statement document she had printed earlier.

"Sometimes something really jumps out," she says and points out that his income for one month in 2016 looked high compared to other months, but the tax that he paid was not much higher. She checks the employer details in FIRST. She opens the EDSR application, looking for tax returns.

While waiting for EDSR to open, she starts to clear some papers on her desk. However, at this point, the PC she is using suddenly turns itself off. She calls a colleague over to help her change her phone status to 
unavailable while she restarts the PC.

While waiting for the PC to restart, Brenda starts highlighting the customer's income on the physical bank statements attached to the application form. She spots a payment to an airline, "I wonder where the mother lives". Meanwhile, her PC has restarted; she starts by logging into FIRST.

At that point the phone rings, but when she answers it, there is no one there, "Oh they hung up. I thought I was going to get one". She redials, "Oh now it's engaged".

She returns to searching the tax returns in EDSR. Returning to the FIRST system, she sees that the customer lives in Auckland and the mother of his children lives in Wellington; she makes a note on the Disclosure of Financial Statements to justify the airline payments. She continues checking the customer's bank details highlighting his rent payments. "He's not an excessive spender".

Once she has reviewed all his bank statements, she explains "that's about it really. I can't talk to him until I'm off phones".

Both Vignette 6.3 and Vignette 6.4 illustrate the multiple systems and sources of records that the Debt 2010 staff used. While the dominant source of records was digital, mostly managed in FIRST, in Vignette 6.4 Brenda indicated that she preferred to work with physical records for note-taking and analysis. Both vignettes also demonstrate that although the primary source of records was digital, the systems they worked with required manual processes to integrate details between the ICTs, for example, copying and pasting DLN numbers in different FIRST screens 
and to LOLA for generating new letters. During the observations, on any one occasion, participants would have ten or more different FIRST screens open, navigating through each screen to locate different details. Each interaction with members of the public was entered in a separate FIRST screen, authoritative details about the customer was a different screen, as were details on payment history, relationship histories, and other personal details, each interaction also generating unique DLNs. As described by Laura:

We have a system called FIRST, which is, it's quite, it's like really basic, but not basic. I don't know how to describe it. Like you look at it and think it's really simple, but it's not. It contains a lot. And to be able to input so much information, yeah.

(Laura, Inland Revenue)

The following vignette, Vignette 6.5, describes the process of entering details from an interaction. Officers required comprehensive details about all interactions, but the time to enter the details is limited, and it could be difficult to summarise complex and lengthy conversations. The front-line staff have set time after each call to enter notes from the call into FIRST, which is referred to as 'Wrap up' time. 'Wrap up' time is accounted for separately from the actual call time and from 'Follow-up' time, which is used to complete any necessary tasks in response to the call and staff note this differently in the work activity monitoring system. Vignette 6.5 describes Courtney during a phone shift, the call that she receives is a member of the public who has some Inland Revenue debt, and during the conversation, Courtney sets up an arrangement for repaying the debt.

\section{Vignette 6.5: Arranging debt repayments}

A phone call comes through, which Courtney answers straight away using the SVOC system. "Recovery Team, Courtney speaking”. The IRD number of the customer did not display in the SVOC system so she 
manually enters it as the person states it and asks a series of validation questions, she then selects the 'Passed' option in the SVOC system. She makes some brief notes on paper and is flicking between various FIRST screens on her computer very fast as the person is speaking to her. She also uses the calculator on her desk.

Once Courtney confirms the payment arrangements, the person has another question for which she needs to transfer the individual to a different IRD team for an answer, although she is not sure which team. So she puts the customer on hold and calls the Inland Revenue Business team. She explains the situation. "Oh that was lucky, they could help," she says out loud as she transfers the customer.

She then returns to FIRST and starts updating the 'Action Summary' screen. The phone call took approximately 10 minutes, including validating the individual and putting them on hold briefly. It takes Courtney approximately 5 minutes to write up the notes in FIRST, where she documents a detailed and comprehensive summary of the call, based the handwritten notes that she made during the conversation.

As described by Courtney and Brenda during their interview, the details captured in FIRST is dependent on the efforts of the Inland Revenue staff member, and there can be variations between officers, which can cause frustrations for front-line staff:

If you get a copy of bank statements, some people will put every transaction in... and others will put in sources of income, bank account and address. An immigration reply, like if we sent a Section 17 request to Immigration, I always put in all the passport numbers, all the departure dates, and all of the arrival dates, but some people think only the last few are relevant, so they only put in the last few. 
(Brenda, Inland Revenue)

\subsubsection{Community Compliance}

The third unit involved in the Inland Revenue case study was Community Compliance. The role of the Community Compliance unit was to support people and businesses to meet their tax obligations by providing information, reminders, selfassessment tools and online services (Inland Revenue, 2014d). The Community Compliance Officers offered free tax education and advice to any individual or organisation in business, anyone considering setting up in business, and non-profit organisations. Community Compliance Officers also identified potential noncompliance with tax requirements and implemented remedies to address the situation (Inland Revenue, 2014d). In 2013-2014, Community Compliance Officers engaged in 41,896 customer contacts, including visiting 118 mobile office sites and delivering 3,858 seminars, expos, workshops and appointments (Inland Revenue, 2014b, p. 17).

One of the participants, Rachel K, described her role as a Community Compliance Officer:

I do a variety of things. So, I do education, like seminars, one to one advisory so educating people on their tax obligations like for a range of GST, PAYE, income tax... And then I also do enforcement work, so that's like investigation type work. So people who aren't complying with their tax obligations, umm... encourage them to make voluntary disclosures. And then I manage a portfolio of tax agents.

(Rachel K, Inland Revenue)

\subsubsection{Identify potential non-compliance with tax requirements}

Rachel $\mathrm{K}$ had been in her role for a little over one year at the time of the observation, before this role she had been an Investigator, and prior to that position, had worked 
in an Inland Revenue call centre. She had worked for Inland Revenue in the regional office for five years in total. There was a team of four people from the Community Compliance team within the regional office working on this initiative, and they planned to visit the companies the following Tuesday, in pairs. Before any visits, they collated details about each company and completed pre-check forms, which provided a snapshot of relevant details, including directors details, financial and tax returns and any previous tax-related investigations. The officers would not show the pre-check form to the customers or take them into the customer's premise; they would review the details immediately before the visit and afterwards, when they would add comments. The officers would then scan the completed forms, save the files to a folder on the shared drive and print them; the officers would attach a sticker with a DLN number to each physical copy, sending them to the Processing Centre for filing. Each officer would also add details relating to the visit in the eCase system and register the DLN number and corresponding physical form in the FIRST system.

This vignette describes Rachel K working with a colleague, sitting at her desk, preparing for visits to local companies to review their tax situation.

\section{Vignette 6.6: Preparing information for company visits}

At Rachel K's colleague's desk, there is a spreadsheet open on one monitor, and on the other, they are searching in FIRST to identify IRD numbers. They are looking for one particular company but cannot confirm the IRD number, so make a note in the spreadsheet indicating this.

They move onto the final company listed on the spreadsheet and search for it by the name in FIRST. The search returns a number of results. They 
work through each to identify the company they are looking for, at which point Rachel K's colleague, who is 'driving' the PC, opens the Company's Office website to confirm the names of the directors. They check that the directors' details are the same on the Company's Office website and in FIRST and then update the 'Confirmation Date' field in FIRST. Rachel K's colleague comments that she always does this, so she "knows it has been done".

Rachel K explains that they have pre-check forms to complete, although for some of the companies they already have these prepared from a previous initiative earlier in the year. She directs her colleague to where they are on the shared drive and sends these scanned documents to the printer. They calculate that there are six pre-check forms to complete. They return to the shared drive to locate the pre-check form template, update the date in the footer of the document and then print six copies. They also print the spreadsheet with the list of companies the team will be visiting.

At this point, Rachel K's returns to her desk to complete the forms by searching FIRST and eCase for the necessary details. However, the forms did not print so Rachel K opens the form template and prints them again.

Once the forms are printed and collected from the printer, Rachel $\mathrm{K}$ logs into FIRST, searches for the first company on the list by IRD number and starts completing the pre-check form by hand.

This vignette describes how Rachel $\mathrm{K}$ and her colleagues planned to use tax-relevant records and customer details when travelling outside of the office, where they would 
not have access to FIRST or any other Inland Revenue business systems. This vignette also reinforces the role of paper as a format of ongoing importance, as the team would not have access to laptops or other devices and, therefore, access to Inland Revenue business data when visiting the companies. Hence, it was necessary to prepare these details in the physical format in advance of the scheduled company visits.

Related to the access to records when outside of the office, is the importance of protecting tax secret information. Staff are very limited with what customer details they discuss or share, including to colleagues, and this extends to records taken outside of the office. The pre-check forms that Rachel $\mathrm{K}$ and her colleague were preparing in Vignette 6.6 would not be taken physically into the customer's premises. Rachel K described this organisation-wide approach to secrecy in the interview with her as:

You're not even allowed to tell whoever you live with where you are going. You know you might be going away for work for you know a week or something and all you can kind of saying is I'm going away. You're sort of allowed to say I'm going to you know what place but you're definitely not allowed to say what you're doing [...] Like if you breached secrecy, you'd lose your job.

(Rachel K, Inland Revenue)

\subsubsection{Implement remedies to address a non-compliant situation}

This vignette describes an observation with Aroha, a Kaitakawaenga ${ }^{11}$ Māori officer; the focus of her role was as a liaison with Māori communities. During this particular observation, Aroha was preparing a submission document on behalf of a Māori nonprofit organisation to request adjustments to a penalty that was overdue. This vignette also describes Aroha preparing a report in Microsoft Word; unlike other

\footnotetext{
Kaitakawaenga is described as "mediator, arbitrator" by the Māori Dictionary (online) at www.maoridictionary.co.nz (accessed 1906 2017).
} 
units within Service Delivery, staff within the Community Compliance unit created documents outside of the LOLA system.

\section{Vignette 6.7: Preparing a submission report}

Aroha has a quick look around her desk and explains that she is checking that she does not have any customer details on her desk.

She has two monitors. On one monitor is the Microsoft Word document that Aroha is writing. The second monitor displays a pdf document. The Word document that she is working on is a report template with predetermined headings, each followed by explanatory notes in grey text to be removed.

She opens the Inland Revenue Knowledge Base and completes two searches, locating the item she is looking for on the second search - she opens it and checks the policy tab and then starts adding points to the Legislative References section of the Word document she is working on.

At one point, she clicks on a shortcut for 'Stickies', which opens some digital Post-it notes saved on her desktop. These are short pieces of information relating to, in this case, abbreviations for tax-related terms.

As well as demonstrating the clear desk policy, ensuring that no tax or customer details were left on her desk, this vignette also illustrates the approach that Aroha used to manage ephemeral documents. Unlike the other Inland Revenue participants who used pieces of paper and notebooks to note short, temporary details and reminders, Aroha used digital notes that she placed on her desktop. In the interview with Aroha, she explained that she preferred working with digital records, rather 
than physical records and this was further demonstrated when examining her physical working environment. Her desk appeared like she was sitting in a temporary desk, although she was not, as there was no paper on it at all. There was one empty in-tray to one side, labelled 'Inbox of Aroha. Please place inwards mail on top'. There was also a shelf on the other side of the desk, which contained one sheet of the pink DLN barcode stickers. Aroha explained that as part of her role she travelled extensively around the region, with access to a laptop, which contributed to her preference for digital records, rather than needing to carry physical records with her.

\subsection{Discussion}

Based on the recordkeeping behaviours observed during the fieldwork, two themes were identified:

- Inland Revenue front-line staff were highly motivated to manage and protect customer details; and

- Staff made concessions for the limitations of ICTs.

\subsubsection{Front-line staff were highly motivated to protect customer details}

One theme identified during the case study is that staff were highly motivated to manage and protect customer details. Staff described how they felt that they were required to protect customer details to a higher degree than other organisations, public or private, and that this influenced whom they discussed personal details with, what records they created and captured, how those records were captured. All participants reflected this sentiment. 
Karen described why she felt personal details held by Inland Revenue about customers needed a higher level of protection than any personal information managed by other organisations:

I think that with the type of work that we do, we have to be a little bit more aware because of the breaches of privacy. And we are seen as government employees to be held up, and looked at a little bit more harder than other employees... when we do something wrong, the implications are far greater.

(Karen, Inland Revenue)

Validating customers, to confirm whom the officers were speaking to, either through the voice identification system or manually using a series of questions (and registering the responses in the phone system) was standard behaviour during customer interactions. For example, during one observation Deanna needed to contact a customer, and although she had initiated the phone conversation with the customer, she still asked her a series of validation questions and explained it was important that she did this to confirm she was speaking to the right person. Validating customers to ensure staff were speaking to the relevant person and sharing appropriate details directly related to the requirements outlined in the Code of Conduct. Laura described these responsibilities as:

That's the first thing in your mind, as soon as you start talking to someone. Is this the right person? What am I actually telling them? Can I tell them that? It is really important. Because its people's lives, especially with child support. It's very sensitive issues for some people.

(Laura, Inland Revenue)

Staff appeared highly motivated to capture details about all engagements they had with customers. During the interviews, some participants mentioned how important it was to have extensive notes of interactions with customers, and similarly mentioned their frustrations if there wasn't sufficient detail in the customer's history 
to understand previous interactions. Many participants explained the need for comprehensive notes from a predominantly personal perspective, as it was difficult to understand what other officers may have previously said to the customer. Aroha also described the need for comprehensive notes of all customer interactions from a broader, organisational perspective:

If you've got a non-compliant customer, not just Joe Bloggs that makes mistakes, but a really non-compliant customer you know the Joe Public wants us to concentrate our efforts at him rather than targeting a small business person, so if there is a history recorded of this person, you know it makes it much easier to throw the full force of the Act at that person, but if we've been slack on the recordkeeping and details in the system... [fades away]

(Aroha, Inland Revenue)

Participants discussed how they captured details of customer interactions, in particular, that other parties were not mentioned by name, in case there was an OIA request:

You've got to be very careful about the company records, that you only record stuff relating to the company. A wife, you record what's relevant to her only, not her husband. And then husband under him.

(Brenda, Inland Revenue)

The focus on how customer details were captured also extended to ensure staff did not capture their personal opinion of the situation within the customer notes:

That's why we have to be mindful of what notes we put in the system [...] We can't go, "oh that guy's an arsehole and there's no way he's going to survive in business" because they could request information under the Official Information Act.

(Dawn, Inland Revenue) 
The fieldwork highlighted that participants focused on supporting the broad organisational aim of maintaining the integrity of the tax system and that the primary way they contributed to this was through their approach to managing customer details and upholding the secrecy provisions of the TAA.

\subsubsection{Staff made concessions for the limitations of ICTs}

The second theme identified during this case study relates to the way staff used the organisation's ICTs during interactions with customers and how this influenced their recordkeeping behaviours. One of the most significant implications for staff was that firstly, to complete many single tasks there were the multiple systems that they were required to use, and secondly that these multiple systems were not integrated. For example, staff used the LOLA system to generate and issue letters based on pre-defined templates populated with details maintained in FIRST or other Inland Revenue systems; staff then used the FIRST system to capture details that they had created the letter and that they had sent a letter to customers using LOLA; and, to view the letters that had been sent to customers staff used the EDSR system. These were separate systems, requiring separate login processes, had different interfaces with no, or limited, similarities between the functionality or the look and feel, and no integration of data.

As previously discussed, the core business system, FIRST, was an MS-DOS system implemented at Inland Revenue in 1991. As a result of the outdated system functionality, staff were required to interact with this system in manual ways. For example, staff selected details in one FIRST screen, they then copied details and pasted them between FIRST screens (e.g. IRD numbers, customer names, children's names). Staff often completed calculations using a physical calculator or referring to online calculators in the Knowledge Base, and then manually entered the details into FIRST. 
During one observation, as Brenda finished a call with a customer, she needed to enter a series of payment details for a debt in FIRST. Once she completed entering details of each of the monthly payments in FIRST, she printed the details. She then created a new letter using a LOLA template and entered the details from the printout of the FIRST screen. This process involved entering 28 different payment entries individually to the letter. Once Brenda completed this step, she then spent quite some time carefully checking the details were correct. The risks, particularly in relation to data and human error, associated with these manual methods and multiple systems are evident; in addition to the time taken by staff to manually complete and review the actions.

Participants also mentioned the use of email to communicate with customers; in particular how this was fraught with challenges. Courtney explained during an interview:

We also get emails from people who realise our email addresses, because as long as you've got that dot in between your name, we can get emails like that. Those ones we usually call and say look we can't respond via email, this is the number you need to ring.

(Courtney, Inland Revenue)

Inland Revenue staff could use MyIR online public-facing website to communicate with customers. However, the customers were required to initiate the correspondence. Moreover, as Brenda described, "they can't send attachments so if they need to send us forms or anything they'll send them to you directly". Sending tax sensitive records using email was not recommended by Inland Revenue as this situation introduced security concerns, so the front-line officers would explain the risk, offer alternative methods to the customer and officers would not respond via email. Courtney highlighted a further potential challenge using email to interact 
with external parties that: "the only gap in the net would probably be if people are receiving emails and they don't lodge them".

The 2014 Annual Report (Inland Revenue, 2014b, p. 134) listed privacy breaches, including where Inland Revenue staff sent account statements and customer details to the wrong tax agents. The report explained that the main cause of breaches was as a result of staff manually handling correspondence; although as outlined above, the ICTs environment necessitated staff to handle correspondence and other forms of customer details manually.

In summary, participants worked within an ICT-enabled environment where the technical infrastructure was outdated, unintuitive requiring significant induction training for new employees, and systems were not integrated requiring manual tasks to transfer information between systems, thus introducing potential for human errors. 


\section{Focus group findings}

\subsection{Introduction to this chapter}

The focus group is a qualitative data gathering technique whereby an interviewer systematically questions several people simultaneously (Fontana \& Frey, 2005, p. 703). This study conducted focus groups to explore macro-level perceptions associated with the individual recordkeeping behaviours identified during the case study fieldwork. The three focus group meetings occurred between July to August 2016 with 23 participants in total attending (refer to Section 3.4.2 for details on the focus group discussion protocol and invitation process and Appendix D for the list of participants).

Eight recordkeeping professionals attended the first focus group meeting, held in July 2016. The researcher facilitated this session as part of a monthly meeting of recordkeeping professionals in Wellington, New Zealand; the organisers publish open invitations each month to the meetings on the New Zealand recordkeeping email distribution list, and all interested can attend. As well as exploring macro-level perceptions of individual recordkeeping behaviours, this focus group meeting discussed specific aspects of the case study findings to ensure the findings were relevant and reflected the participants' perceptions of front-line public servants recordkeeping behaviours. The six women and two men in the first meeting all worked in Wellington, New Zealand, at different types of organisations: Public Service departments (3), Crown entities (3), and consultants (2).

The second and third meetings were designed primarily to explore the macro-level perceptions of individual recordkeeping behaviours and both of these meetings discussed the same set of topics (refer to Appendix E for the discussion topics). The majority of New Zealand public agency head offices are located in Wellington; 
therefore, the researcher conducted both of these meetings in Wellington, in August 2016.

The four women and three men who attended the second meeting were employed by organisations termed for this research as recordkeeping and information management authorities, which included public service departments (4) and tertiary education institutions (3). These participants taught or instructed others within the discipline on recordkeeping-related concepts, set policy directions for government recordkeeping or monitored recordkeeping behaviours for Archives New Zealand.

The five women and three men who attended the third meeting were managers of information or records management teams or senior recordkeeping professionals, with broad and extensive recordkeeping backgrounds. These participants were employed within a range of organisations, including public service departments (3), non-public service departments (2), state-owned enterprises (1), not-for-profit organisation (1) and consultant (1).

The first section of this chapter presents a summary of the discussion relating to the relevancy of the case study findings from the perception of the focus group participants. The chapter then presents seven overarching themes identified during the analysis of the discussions.

\subsection{Relevancy of fieldwork}

As mentioned, the initial focus group meeting discussed the relevancy of the case study findings, as some years had passed since conducting the fieldwork. During this conversation, the focus group participants agreed that the findings from the case studies remained valid and the participants found the descriptions of the fieldwork observations comparable to situations and the recordkeeping behaviours within 
their organisations. There were some comments amongst the group that while recordkeeping behaviours within the New Zealand state sector had not changed significantly, there had been some changes to the function and approach to EDRMS implementation within public agencies during the five years since the fieldwork commenced:

\begin{abstract}
When you went into organisations probably back in 2009 - 2010, they were still doing a lot of that paper-based stuff, a lot that of manual operational stuff where they were being done by paper-based physical systems, and we've at least moved on in the world now and we have the digital online stuff, which is actually helping - it's more accessible, it's easier, there's less quality checking that needs to be done, things get speed up more.
\end{abstract}

(Rachel S, Focus Group Meeting 2)

Although, as noted within the Inland Revenue case study conducted in late 2014, implementing technological change within large public agencies is time-consuming and expensive. For example, when approved by Cabinet in 2014, the Business Transformation Programme at Inland Revenue, which aimed to modernise the tax revenue system through business process re-engineering, it was expected to take ten years to complete (Inland Revenue, 2014a, p. 42). So, as Rachel S highlighted, while customer-facing forms and processes may be increasingly available online, the backend ICTs that public servants interact with may necessitate using out-dated technology and manual processes. Also, agencies are still required to maintain and support physical processes for those citizens who do not have access to computers, which as described in the vignettes, can reinforce a hybrid recordkeeping environment, introducing complexities for front-line staff.

One participant, Michelle, suggested that the approach agencies took to implement EDRMS had changed during the five years since conducting the initial case study fieldwork. Michelle's role involved implementing EDRMS and ICT-enabled 
recordkeeping systems in New Zealand public agencies, and at the time of the focus group meeting, she explained that she mostly dealt with EDRMS implementations that included integration with other business systems, whereas previously organisations implemented EDRMS as standalone recordkeeping systems:

It's just such a core component of putting a new system in there. People will not now put in standalone EDRMS systems; they have to be a component.

(Michelle, Focus Group Meeting 1)

In summary, the fieldwork findings discussed with the focus group participants were still relevant and comparable to the recordkeeping environment and individual behaviours that these participants understood from their respective agencies. However, there had been some developments regarding the increased and extended use of ICTs within public agencies, both as a means to engage and communicate with citizens and to implement EDRMS that integrate with other ICTs, rather than as stand-alone systems.

\subsection{Findings}

\subsubsection{The impact of ICTs}

All three of the focus groups extensively discussed the impact of ICTs on the recordkeeping behaviours within public sector agencies, and this section describes four sub-themes identified within the broader theme of the impacts of ICTs on public sector recordkeeping behaviours.

\subsubsection{The nature of recordkeeping in ICT-enabled environments}

Participants discussed how the nature of records and recordkeeping systems have become increasingly complex in ICT-enabled environments. The participants explored this concept by discussing some of their experiences where organisations 
had excluded records created and captured in business systems as part of their organisational recordkeeping system:

When we did the audit program, going in and explaining to record managers that it wasn't just their Objective system. I had one organisation who shall not be named, but they didn't want, they did the whole audit program, without including their GIS data, which was a large section of what they did and when it came down to it, and we asked the information, and they were horrified that we would even include that as being records management stuff.

(Rachel S, Focus Group Meeting 2)

Participants discussed how this opinion that organisations only manage digital records within the EDRMS and not in other ICT-enabled solutions also exists amongst recordkeeping staff:

Even the record managers say "No that's not my job". I am only responsible for this nicely controlled area.

(Gail, Focus Group Meeting 2)

Participants also discussed the ongoing impact of poor implementations of recordkeeping systems when organisations first introduced ICTs and began transitioning from purely physical recordkeeping systems. This discussion described situations similar to that described by the United Kingdom's Cabinet Office (2017, p. 8) where public servants saved digital information in their own ways, rather than using agency systems.

They had this team of people [...], and they managed these things, they knew where everything was, and they kept everything, and then I think that the technology came along and they went, "oh the technology will do it". And so everyone just kind of stopped and no-one did anything and, everyone managed their own in their own way with their own little, and all of a sudden people said: “oh, actually we don't even know what we've got anymore". 
(Sadie, Focus Group Meeting 3)

Sadie also suggested that there may be some relationship between these historical approaches to managing digital records and a consequential lack of trust by public servants towards digital records and digital recordkeeping systems:

And people don't trust only electronic files. So even if you set up processes and standards, "Oh but I'm still going to do my own" - "I don't believe you".

(Sadie, Focus Group Meeting 3)

Julie U suggested that there are considerable differences between physical files and digital equivalents, reinforcing her opinion that organisations and individuals approach physical and digital records differently:

I mean if you take an all paper file, what astounds me is how beautifully organised it is. And there's no crap in there, excuse the French. You take an equivalent electronic, and there is mess, and there's no - the context is all over the place, and it might be in date order from the time they put it in; doesn't necessarily mean it's the correct date order, and so you know you're not dealing with the same thing.

(Julie U, Focus Group Meeting 3)

Participants also discussed the tangible nature of physical records, which they viewed as a strength of physical records, an element missing from digital recordkeeping:

There is nothing quite as satisfying as perusing the files, is there? Well, you know you print it out, the 2-hole punch, put it on the shelf.

(Mark, Focus Group Meeting 1)

Participants felt the changing nature of recordkeeping could partly be attributed to the tangible nature of physical records and historical approaches to digital 
recordkeeping resulting in public servants not trusting digital recordkeeping and ICTs solutions. Also, participants discussed the lack of clarity received by public servants from organisations and recordkeeping professionals about what is a record, as well as where and how they should be managed.

\subsubsection{Digital and recordkeeping literacy}

There was an interesting discussion amongst the participants at the second focus group meeting regarding the digital literacy of public servants, which the participants felt impacted individuals' abilities to conduct recordkeeping behaviours. Gail explained that it could be difficult for recordkeeping professionals to assess the digital skills of staff:

\footnotetext{
It's actually got much more difficult to actually assess people's level of digital literacy competency understanding because the interfaces have got so much better and people can do things but actually whether they understand the consequences of what they are doing with, where they're saving things, is just another matter of time.

(Gail, Focus Group Meeting 2)
}

Participants also discussed that, beyond the technical abilities to use the systems, some public servants do not understand why they are expected to use different ICTs within the workplace and at times are not provided with the appropriate time to fully appreciate the role of the ICTs within the scope of their day-to-day work. The focus of many ICTs implementations is on 'how-to' training, without reinforcing the broader objectives of using particular ICTs, and that this has consequences for recordkeeping behaviours:

I don't think people have the time to get to grips with these new big systems that are put in, they are sort of touted as being really simple but people don't find them simple and they don't have the time to really embrace them and come to grips with them and have the confidence with them. 
(Helen, Focus Group Meeting 2)

Participants also discussed that digital literacy is different to recordkeeping literacy:

Well, I mean a classic example is text messages, and people's lack of understanding that if they write the business text message it has actually become a record.

(Rachel S, Focus Group Meeting 2)

I have found that a lot of those who are digital literate, so you know, your tech, sort of IT experts, etc., still have this block that recordkeeping, information management, etc. is more than just IT. They still don't quite get it and still come up with, "oh yeah, the system will do that". But, there is a whole heap of other stuff around process, behaviour and culture and all manner of things you actually need to factor in here. (Henry, Focus Group Meeting 2)

Participants discussed that the rate of change in relation to ICTs in comparison to approaches to recordkeeping have potentially impacted public servants understanding of their recordkeeping responsibilities:

Is one of the things the fact that like there's sort of processes around the paper records was quite, sort of the same for quite a long time but technology changes really quickly? So the moment you've just found a good way to do something, there's a different, better way and so you start battling with that, something else is different and you've got a mix of the processes.

(Sadie, Focus Group Meeting 3)

To a large extent, participants agreed that the digital and recordkeeping literacy of public servants impacts on the resulting recordkeeping behaviours of individuals. Participants suggested that contributing factors to digital and recordkeeping literacy includes the lack of organisational focus on change management and cultural support when implementing ICTs, staff not fully appreciating recordkeeping implications of ICTs behaviours, as well as rapid and ongoing technological changes. 


\subsubsection{Changing nature of public sector work and the use of ICTs}

As discussed in the literature review, ICTs have introduced changes to the working environment of public servants, including the delegation of responsibilities to create and manage public records to individuals. Participants discussed that some public servants might resent that responsibilities to create, manage and use records have been delegated to individuals, whereas previously secretaries, records clerks and records managers would have been responsible for these processes on behalf of the public servants:

Staff feel that that's not their business. They don't do records management - they do their job.

(Anna, Focus Group Meeting 2)

Michelle also discussed the changing responsibilities of public servants to manage records in ICT-enabled organisations, as she relayed a situation at an organisation she worked with. The organisation was identifying who was responsible for capturing details from a specific type of business activity into ICTs. This particular business activity occurred between public servants and members of the public outside of the workplace:

We actually sort of went back to basics and said well, first of all, they struggle to use a computer. So is it fair to expect them to? [...] So it came right down to responsibility and you know if these guys are producing the information, is it also their responsibility to manage it? We had lots of big discussions about it. It was quite interesting. In the end, we went back to "no, it is their responsibility" because there is some interpretation required, you know, you can't just hand it over like a bit of typing. Because they know what the information is, they understand where it has come from; they understand the context. You know, so it was actually their responsibility to process it, store it manage it properly, get it into the right place.

(Michelle, Focus Group Meeting 1) 
In addition to changing responsibilities to include the creation, management and use of records, participants also discussed how public servants' use of ICTs at home could influence their recordkeeping behaviours and attitudes towards ICTs in the workplace:

In the home environment they are very much relying on the technology that is coming to them from service providers that is the latest technology, whereas, in any organisation, this isn't just government, of course, any organisation, you're constrained by technology of that organisation, which may or may not be as up-to-date as what you have at home.

(Henry, Focus Group Meeting 2)

As a further consequence of how public servants' use ICTs at home, public servants may begin to use emergent technologies at the workplace without the necessary approval or input from the ICTs staff. Informal introduction and use of ICTs has implications for both IT managers and records managers:

The technology bottleneck used to be an opportunity for information managers to get involved if they heard about it, but now even that bottleneck is beginning to disappear, and you hear - it's very interesting, you hear, my IT colleagues saying the kind of stuff you would have heard an IM manager saying five or 10 years ago: “Ah, how have they gone off and do this without consulting us?".

(Scott, Focus Group Meeting 3)

In summary, the focus group participants agreed that recordkeeping responsibilities have changed with the introduction of digital records, where tasks that may have been conducted by office support staff are now required to be performed by the public servants who created the records or conducted the business activity. The focus group participants also felt that the ICTs that staff may access and use at home influenced behaviours in the workplace and that this may result in expectations placed on organisations to make emerging ICTs available in the workplace. The 
process to initiate the use of emergent ICTs, sometimes without input from technical or recordkeeping professionals within the organisations, also needs to be considered.

\subsubsection{Designing and implementing ICTs}

Participants at the three focus group meetings discussed ways in which the design of the ICTs and the implementation of these ICTs can impact the recordkeeping behaviours within organisations and across the broader public sector in general:

We've gradually digitised various bits of that system, but at different times for different purposes and it doesn't fit together properly. We still have that data layer at the top, but all the content is managed really badly, often, and so if it's like where we've got to build - we've got a shanty town. I mean I think it is probably applied to the whole public service, there are shanty town systems. Some bits of it work really, really well. And the more structured the data probably the better the system works, but it's not a cohesive system.

(Perry, Focus Group Meeting 3)

This led to further discussion within the third focus group regarding how the design and implementation of ICTs can support recordkeeping within public agencies:

But you know, it's designed from the ground up to be mobile, to be digital and you know, recordkeeping is built into that, but that's just a wee part of it. It's actually - it's not just recordkeeping. The whole service has been designed, has been rethought... That to me that's the future; that's how things will work.

(Perry, Focus Group Meeting 3)

Scott raised a further point about the use of multiple recordkeeping systems across the New Zealand state sector:

Hong Kong uses one system. China, with all its population, uses one system. New Zealand agencies are just fragmented.

(Scott, Focus Group Meeting 3) 
Participants also discussed significant differences between how the context of physical and digital records is captured, which has significant design implications for ICT-enabled recordkeeping systems:

The context has to be consciously applied in the way that it didn't quite have to be, it was embedded within the process and paper processes; that has not been embedded within digital processes in quite the same way, it has to be conscious, it has to be applied through metadata and process and other means, and that process has been delaminated from the content layer. What happens is you get content creation without the context layer having been applied, and in fact sometimes the context layers didn't exist or haven't been recorded anywhere.

(Scott, Focus Group Meeting 3)

Ryan also suggested that where ICTs are not designed or implemented effectively for the end user, public servants can be complacent about where they store documents in the workplace, potentially storing records external to the recordkeeping system:

And there is a reliability brought about in, with technology, especially with personal desktops where I think you can get little complacent about storing stuff locally on your hard drives instead of in a content management system.

(Ryan, Focus Group Meeting 2)

The focus group participants agreed that the approach to designing and implementing ICTs has impacts on how public servants create, manage and use digital records. The description of the New Zealand state sector's approach to recordkeeping systems, including at a broad 'all-of-government' level and at an organisational level as fragmented and a 'shanty town' by recordkeeping professionals is interesting, especially as the public servants expected to use these ICTs on a regular basis must also be challenged by the fragmented nature of these ICTs. 


\subsubsection{Managing records within the public sector}

The second overarching theme identified during the analysis of the discussions was differences between recordkeeping responsibilities in the public and private sectors in New Zealand. There was much discussion during all three focus group meetings regarding the nature of the public sector and fundamental approaches to recordkeeping:

Mainly they [the private sector] see the value of information much easier than we do in public sector.

(Henry, Focus Group Meeting 2)

The other thing with private sector is they actually monitor and they report, and they do all those sorts of things on their information and what they doing with that, and I think the private sector they actually understand better that they're creating information for their organisation, whereas, as we've just discussed - public sector "it is mine. It's mine I am not letting it go".

(Rachel S, Focus Group Meeting 2)

There was further consideration amongst the participants that the drivers for recordkeeping were different between the two sectors:

Well, if you don't manage your information correctly then you're not going to get ahead of your competitor, so therefore you are not going to make any money. (Rachel S, Focus Group Meeting 2)

The groups also discussed the nature and culture of the public service and the suggestion that recordkeeping is a core responsibility of all public servants:

And I think there's almost a breakdown of 'I'm a public servant and I somehow have some kind of responsibilities and my stuff is owned by the Crown'. I've been running of seminars for some new hires, and they have no concept. And "I'm like, oh gosh" so I have to do sort of the public servant 101 [training]. Because "It's mine, I created this 
email, no it's mine". "No, it's not actually". And you know, right, that's basic? So, if they think it's theirs they might consider it their Outlook, they think they can save stuff in the cloud, they think they can do whatever they like with their information so that the whole concept of 'you're part of this crown-funded public service' is just gone. (Julie U, Focus Group Meeting 3)

There was also some discussion about challenges sharing information between government agencies, beyond the technical considerations of system interoperability and metadata:

Yes, technology can do it, but our processes, our way of thinking about the information, how it can be used, our way of actually creating in the first place so can be used in multiple ways. We haven't gone onto that, we have not moved on yet. (Henry, Focus Group Meeting 2)

The participants also discussed that the ways that governments interact with citizens and businesses is changing, mostly as a result of the increasing use of ICTs:

You know when we think about maybe intellectual property systems or something like [agency name]; you know that's a real example of how an information system could actually work, can actually transform the way citizens interact with government, or businesses interact with government.

(Perry, Focus Group Meeting 3)

In summary, the focus group participants agreed that there were fundamental differences between what it meant to create, manage and use records in the private sector and in the public sector. There was a consensus that while the recordkeeping behaviours of individuals and the recordkeeping systems may be similar between the two sectors, the sectors were underpinned by differing drivers for recordkeeping: competition in the private sector and compliance in the public sector.

\subsubsection{How public servants manage records}


The third theme identified during the analysis of the focus group discussions was that the nature of recordkeeping within the public service had changed, including the composition of the recordkeeping teams within public agencies:

I mean that's the other way that it has changed, over the years you had very separate processes for doing records management and nowadays it is actually intermingled and we are encouraging it to be intermingled within those business processes so that you don't have a forty man records management team anymore.

(Rachel S, Focus Group Meeting 2)

Mark suggested that differences in work styles within the public sector influenced recordkeeping behaviours, particularly in relation to process-driven roles where recordkeeping behaviours have the potential to be embedded into business processes:

There's two types of office environments, office culture or office workers. There's the very process-driven... passports, they're very process driven. There's steps you need to take just like that. Everything's sort of embedded, and then there's I don't know, policy analysts and stuff like that, who... If they chuck the report in at the end, that's good enough and won't touch the EDRMS for weeks on end, that sort of thing. But their work environment is very different. You know, their work process is very different.

(Mark, Focus Group Meeting 1)

Participants suggested that certain roles or personalities have different approaches to recordkeeping, in particular within ICT-enabled work environments:

You have got a lot of old-school people. Especially in my organisation we have a lot of solicitors. So legal personalities, they love paper. They would print off emails, so every email would be printed off and we [also] do have an enterprise vault so it archives any emails that come in and out of our organisation.

(Jennifer, Focus Group Meeting 1) 
Participants at the third focus group meeting discussed public servants as representing a unique subset of employees and suggested that the core values of public servants, or what it means to be a public servant in New Zealand, is breaking down:

\footnotetext{
There's little concept within New Zealand of the public sector. It seems people are more about working on their own. In some countries, people will often, you know on the departure cards, put themselves down as public servants. Whereas in New Zealand, you're less likely to consider yourself a public servant, the organisation or even your team is the most important component.

(Scott, Focus Group Meeting 3)
}

Overall, participants provided interesting illustrations of the ways in which the nature of the public service and the behaviours of public servants influence recordkeeping behaviours, including based on specific types of work, roles and personalities. Although, the participants also questioned how relevant this is within the current public sector environment, suggesting that the values of public servants are changing.

\subsubsection{The influence of records managers on staff recordkeeping behaviours}

Participants discussed their ability, as recordkeeping professionals, to affect the recordkeeping behaviours of staff within their respective organisations, reflecting the fourth analytical theme. One of the factors raised was the impact of technology, where organisations devolved recordkeeping responsibilities to all staff enabled through the use of ICTs:

And we know how it should and could be, but how to get it there and all of those people, because they're all doing it in their own way, in their own - it's just, you don't have the same actual level of control that you somehow did have. People behaved better with paper for odd reason. 
(Sadie, Focus Group Meeting 3)

One of the participants, Jennifer, discussed how staff printed records as well as storing them digitally in the organisational EDRMS and how she influenced a change relating to this behaviour:

When I first started, they [staff] were very heavily printer-based, and because I was the only one there, I couldn't keep up with the amount of filing ... and I'd say, "why are you keeping this printing?" I said, "we have got an EDRMS", and so, yeah it has taken me about five years, but I've got them to stop handing over paper filing. So we have kind of come to an agreement, anybody who hands me something, I'll go "Is this saved on the document management system?" and they will say, "Yes". I'll go "Well we don't actually need to keep this". So they have gradually learned that they can keep their own records on their desk, and I'm happy with that.

(Jennifer, Focus Group Meeting 1)

\section{Another participant discussed how a previous manager of hers had built relationships with specific parts of the organisation to influence recordkeeping behaviours with senior managers:}

I had a manager some years back who got inside with the company secretary. I just thought that was most amazing information strategy because what does this company value the most? And that's what she spent her time on ... it's how you're using your time and how you apply your expertise.

(Karen, Focus Group Meeting 1)

There was also discussion regarding the use of policies and procedures to communicate recordkeeping behaviours to staff:

I think it's often because there are policies, but they are not granular enough, or they don't... there is a grand policy statement, but people find it difficult to actually translate how does that apply to this email or this document. So I think there is definitely a gap for people in interpreting policy and how it actually relates to the 
actual information that they deal with on a day-to-day basis.

(Michelle, Focus Group Meeting 1)

Participants raised questions about the ability of the recordkeeping staff to influence recordkeeping behaviours:

Well, if the repercussions are going to come back to them. You know in the team that I have got things going best with, I still have people who won't do anything until it actually comes down and bites them in the bum and then they will do something about it. There has got to be something in it for them.

(Janice, Focus Group Meeting 1)

Overall, participants felt constrained as to their ability to ensure all public servants within their agencies performed the recordkeeping behaviours required of them, particularly in ICT-enabled workplaces. While the recordkeeping professionals listed numerous tools available to them to influence the public servants' recordkeeping behaviours, including policies, training, monitoring and feedback, as Jennifer explained in her situation, it was personal, repeated discussions about specific behaviours that had the greatest influence on changing individual recordkeeping behaviours.

\subsubsection{The role and nature of the governance framework}

The fifth theme identified through analysis of the focus group empirical data is the role and nature of the governance framework for recordkeeping. While there was consensus amongst the participants that New Zealand required legislation to define and regulate recordkeeping requirements, the participants suggested that the current legislation required some updates:

The Public Records Act needs some TLC [tender loving care] and seriously. And probably I won't make it public records right now and the scheme of things, I would 
make something along 'information management'.

(Rachel S, Focus Group Meeting 2)

Henry suggested that the government needed to clearly set expectations for recordkeeping and information management, before determining any specific updates to existing legislation or introducing changes to the broader governance framework:

But I think the challenge isn't in the legislative framework, it's in the policy settings beforehand... The government gets to set that legislation, so for them and I think where we are at the moment, is getting those policy settings right, understanding what the role of information is, and its value and how it needs to be protected at the same time, so the dual balancing action. I think that really does need some serious work. That will then drive, you know, cohesive changes to legislation, rather than ad hoc changes. (Henry, Focus Group Meeting 2)

The discussion relating to specific updates to the PRA primarily centred around how the PRA relates to other pieces of legislation, including the Copyright Act 1994, the Official Information Act 1982, the Privacy Act 1993, and the Statistics Act 1975:

And that is... the biggest issue. Each piece of those of those Acts, including the ETA, which doesn't really marry up, and some over trump others, and some you really can't work it out because it is too much detail. And, as anyone knows to try and get an update or an amendment within an Act can take anywhere from 5 to 10 years. (Rachel S, Focus Group Meeting 2)

Also discussed was that each agency might have specific pieces of legislation controlling aspects of their recordkeeping environment, in addition to the requirements defined by the legislative framework for recordkeeping outlined within this study. These additional pieces of legislation introduce complexity on the recordkeeping environment unique to each agency. The groups discussed how this 
can introduce concerns over which section of a piece of legislation applies in certain situations:

Yep, there's a few of them out there. Even some little agencies that you wouldn't even think. Like [agency name], they're a classic example, their legislation says something about disposal, I can't remember what and of course, which one trumps which one. (Rachel S, Focus Group Meeting 2)

Participants also discussed how public servants do not need to know specific details about the PRA:

Well, I think the Privacy Act and the OIA have a lot to do with it, and they are well used and well talked about and all the rest of it. The ETA and the Public Records... "what are they?".

(Rachel S, Focus Group Meeting 2)

We purposefully don't mention the concept of records or PRA in any training sessions with staff. They either have lots of preconceived ideas about what records are and what recordkeeping is or get quite flummoxed by it all.

(Julie U, Focus Group Meeting 3)

There was also discussion by participants about the influence of the OIA on the recordkeeping behaviours of front-line staff, reinforcing that staff are aware of the OIA and consequences of information that they capture in organisational recordkeeping systems, more so than the requirements of the PRA:

The OIA changes expectations of public sector employees. Does this mean more or less information is captured? Probably less. Public servants are much more aware that whatever is captured in the system is recoverable under the OIA. We all know that dealing with one or two difficult citizens will change the behaviours of public servants. (Perry, Focus Group Meeting 3) 
In summary, participants agreed that legislation to regulate recordkeeping within public agencies was required, although there were some discussions about specific changes required to make the legislation applicable in ICT-enabled agencies and in relation to other, related pieces of legislation. The groups also discussed that while they may personally recognise that there are updates that could be made to the PRA, the nature of setting legislation is that any changes would take significant time to complete. The key element for the participants as recordkeeping professionals within public agencies was how to communicate specific obligations and requirements to staff in meaningful, easy to follow ways that ensured compliance without necessarily knowing or understanding the underpinning legislation.

\subsubsection{The role of organisational culture}

Organisational culture was a further theme identified during the focus group analysis. Participants within the first focus group meeting explored whether the perspectives of individual public servants altered as a result of the increasing use of ICTs, replacing physical recordkeeping systems and consequently, impacted the organisation's overall approaches to recordkeeping:

\footnotetext{
I don't want to go back to the physical [recordkeeping environment] but walking into a file room and seeing all this stuff was a really good demonstration of you're just one tiny bit in the $\mathrm{cog}$, and everything fits in with the rest of it $[\ldots]$ when you had to take your hand-written piece of paper to the typing pool to be typed, it wasn't yours, it was the organisations.
}

(Anna, Focus Group Meeting 1)

Participants discussed the role of organisational culture to influence staff during the implementation of ICTs, suggesting that insufficient focus is placed on the cultural changes potentially introduced by new ICTs: 
So in designing the implementation of these new systems, how much focus is actually given to that change management, to the culture change, to the processes, to tying it in as much as possible to the workflows, of how people work so they don't have to think in a separate way, how much of... of lack of that may be.

(Henry, Focus Group Meeting 2)

Michelle and Karen both discussed the role of senior management to influence recordkeeping behaviours of individuals within an organisation:

Culture is another thing. People can be doing things great, but as soon as they see one person not doing it, then they all start to wander off as well. You know how it is - if it's tolerated that some people don't have to do it, or the Chief Executive doesn't have to do it then, "Is it a thing, is it not a thing, do I have to do this?" So it becomes unclear and then individual practices come into play a lot more.

(Michelle, Focus Group Meeting 1)

It comes down to the internal culture of what the managers have valued over, over a period of time.

(Karen, Focus Group Meeting 1)

Participants also suggested that there were differences between how public servants within head offices of agencies and those staff in regional offices managed records:

But you guys will know this like, if you are wandering the floor, people will grab you and go "I've got this question, I've been wondering about this for a long time." So you can help them, but if it is regional and we are not there they don't pick up the phone to call you.

(Jennifer, Focus Group Meeting 1)

You know they do not have access to us at their fingertips and wandering around.

(Edith, Focus Group Meeting 1) 
Anna suggested that the size of the organisation and the associated culture may also impact the recordkeeping behaviours of individuals, in particular, the sharing or removing restrictions on records created and captured by one team, so others within the organisation can view the records:

I wonder whether there's an element too about the size of the organisation, whether private sector they're smaller and it is more of a very large team approach, not for every company obviously, but it's a very large team approach, whereas in the public sector, I keep coming across this "but it is our team information why should anyone else look at it?", and all I'm saying is "Well, why shouldn't they look at it?".

(Anna, Focus Group Meeting 2)

Ryan returned to the discussion about differences between private and public sector to explore organisational cultural influences on recordkeeping:

\footnotetext{
I have worked six and half years in public service now and before that in the private sector for three years, and both organisation there were about 30 members of staff, and not once did I think about records [in the private sector role]. Because I had a clear 'raison d'etre' and my 'raison d'etre' in one of the organisations was doing first and second line support for an IT firm creating risk management software and the second job was a software developer. Someone else, I imagine, took care of the recordkeeping. (Ryan, Focus Group Meeting 2)
}

In summary, participants at all three focus group meetings acknowledged the important role that organisational culture had on the recordkeeping behaviours of individuals. Specific factors discussed in relation to organisational culture included the increasing use of ICTs and how these ICTs are designed and implemented, the influence of senior managers, the location of the team in the agency head office or within a regional office, the size of the organisation, as well as the position of the organisation within the broader public sector.

\subsubsection{Recordkeeping and risk}


The risks associated with recordkeeping was another theme identified during the analysis of the three focus group data:

It's a total concern with my organisation because I've worked in plenty of organisations where this is a huge factor. I think there is a tendency to overprotect.

(Edith, Focus Group Meeting 1)

Participants discussed challenges communicating risks associated with recordkeeping within their organisations:

It doesn't matter how many times they tell people, 'if you get hit by a bus tomorrow, we're stuffed', and they don't believe it's gonna happen.

(Edith, Focus Group Meeting 1)

A DHB I worked at, risk was again a big thing. But it came in waves, so when there was a leak, then it became a huge big thing. But it was never by fear or intimidation, it was just more of 'remember you're a professional'.

(Michelle, Focus Group Meeting 1)

Sadie recounted how some public servants managed records in an extremely riskadverse manner, to some extent reflecting the culture within the organisation:

And in our organisation, some people still think absolutely everything has to exist in paper as well as electronic, and some don't. So - and so there's almost like a triplication of paper just in case, because I don't trust anybody, and then sometimes there's nothing at all.

(Sadie, Focus Group Meeting 3)

Participants also discussed how the public service acknowledges risks associated with recordkeeping and as suggested by participants, risk is treated differently in the public service to the private sector: 
Well, risk management, in general, is only really starting to flourish across government at the moment, in the last decade probably less maybe, much less maybe. But that understanding of the risk and risk-based approaches just hasn't been a central part of how each agency has run itself were I think is much more visible in a private sector context.

(Henry, Focus Group Meeting 2)

And there is a fiscal bottom line, there is a risk bottom line... and I don't think, I mean the public service has risks definitely, but each individual public servant doesn't have that same level of risk as I think you have in private sector, where if you screw up or lose things you are on the line.

(Anna, Focus Group Meeting 2)

To a large extent the participants, as recordkeeping professionals employed within the public sector, appreciated the relationship between recordkeeping and risk. However, the ways that organisations and individual public servants interpreted their roles within this relationship varied. As Sadie suggested, some public servants spend considerable amounts of time ensuring that records are created and captured in multiple formats to mitigate any risks occurring, whereas for other public servants risk is not a factor and they pay little attention to records or their recordkeeping responsibilities during their daily work activities.

\subsection{Chapter summary}

In summary, while a number of years passed between conducting the fieldwork and the focus groups, the focus group participants agreed that the findings from the case studies remained valid and the participants found the descriptions of the fieldwork observations comparable to situations and the recordkeeping behaviours within their organisations.

Analysis of the focus group discussions identified the following themes: 
- the impact of ICTs,

- there are differences between recordkeeping responsibilities in the public and private sectors in New Zealand,

- the nature of recordkeeping within the public service has changed,

- recordkeeping professionals affect the recordkeeping behaviours of staff,

- the governance framework for recordkeeping, while necessary to set expectations at an agency level, ,

- the role of organisational culture, and

- the relationship between recordkeeping and risk.

The focus group discussions highlighted the links between the transactional, administrative, oftentimes invisible, individual tasks associated with recordkeeping, such as creating documents, naming digital files, deleting digital files, etc, and the overall functioning of business teams, organisations, and ultimately government. 


\section{Discussion and analysis}

\subsection{Introduction to this chapter}

This chapter integrates the findings from the three case studies with those from the focus groups, examining, in turn, the three theoretical concepts that make up the analytical lens for this study: recordkeeping informatics, personal information management strategies and the influencing factors on front-line public servants' decision-making behaviours. This chapter also examines the individual, organisational and the New Zealand public sector perspectives on governance in relation to recordkeeping.

The focus of analysis discussed within this chapter shifts from the microrecordkeeping behaviours of individuals explored within the case studies, to the organisational context for recordkeeping and the broader societal approaches towards recordkeeping.

\subsection{Recordkeeping informatics}

The recordkeeping informatics (refer to Section 2.4.2) provides a framework for addressing recordkeeping recognising technological, organisational and societal contexts. Recordkeeping informatics has records continuum and recordkeeping metadata as its two foundational building blocks and specifies three key facets of analysis: organisational culture, business process and archival access.

\subsubsection{Organisational culture}

The first facet, organisational culture, emphasises the importance to recognise and understand the values, attitudes and behaviours influencing information management in organisations, which ultimately reflects the information culture (Oliver et al., 2010, p. 44). 
Participants at both ERMA New Zealand and at Te Puni Kōkiri had the discretion to apply personal values, attitudes and behaviours to their recordkeeping tasks, for example what content staff included within the records and whether staff created records in digital or physical formats, or both. ERMA New Zealand staff placed significant importance on creating accurate records, particularly when interacting with members of the public, where details discussed with members of the public or other organisations could be required by other staff, requested as part of OIA responses or legal enquiries, or potentially misinterpreted due to the scientific nature of the discussions. Participants were observed creating and managing records in both digital and physical formats, as well as digitising physical records. As explored in Vignettes 4.2 and 4.6 of the ERMA New Zealand case study, participants were observed creating the same record in both physical and digital formats when they defined that particular record as important. However, the identification of records to manage in both formats was inconsistent between participants within the same team, and was based on criteria defined informally by each individual.

Resistance to capturing records in the EDRMS was also discussed by participants at both ERMA New Zealand and at Te Puni Kōkiri. Some participants explained that they felt that others within the agency tracked their work within the EDRMS. Consequently, some participants purposely did not save records in the EDRMS until the records were finalised instead using their desktop, personal drives or portable storage devices, thus retaining control over when others had access to those records and applying their personal information management values, attitudes and behaviours to public records (Vignette 4.3 and 4.4 describe Glenn saving records external to the EDRMS).

While all Inland Revenue front-line staff had responsibilities to create records, they had less discretion to apply personal values, attitudes and behaviours to their 
recordkeeping tasks. For example, Vignette 6.1 describes Deanna following prescriptive processes for creating records. There was no EDRMS was in place at Inland Revenue; however, the front-line staff participating in this study were less likely than participants at either ERMA New Zealand or Te Puni Kōkiri to create documents using word processing, spreadsheet, or presentation applications and more likely to use pre-defined templates and notes about customer interactions, which were created and managed within specific ICTs. Participants at Inland Revenue discussed their responsibilities to create accurate records as evidence of interactions with members of the public, and the need to ensure that records of interactions with members of the public were representative of the interactions. For example, Vignette 6.5 describes Courtney completing an Action Summary in FIRST, illustrating the effort she went to capture details of the interaction. Participants at Inland Revenue also attempted to create evidence of interactions with members of the public as soon as they could after the interaction; as described in Vignette 6.5. Both 'wrap up' and 'follow-up' times were actively monitored by managers; if call centre staff took longer than allocated to either document the notes or to complete tasks, managers would address this with the staff. The records created in FIRST were monitored; specific staff were responsible for reviewing the quality of records created by front-line staff. This monitoring activity involved reviewing the completeness of details in the record, whether incorrect or inappropriate private details were included in the record, that staff used the correct codes and that details were created in the appropriate ICTs. If trends appeared in the quality control monitoring where a number of staff repeatedly made the same errors or urgent mistakes were identified, managers provided targeted training at regular team meetings.

Focus group attendees suggested that the use of ICTs raised influenced the information management values, attitudes and behaviours of front-line public 
servants, particularly emergent ICTs that did not integrate with existing organisational recordkeeping systems. The specific example discussed by the first focus group was online chat messages where members of the public were able to engage with front-line public servants using an online messaging tool. The attendees suggested that staff treat these conversations as an equivalent to a phone call and either request the member of the public to email a summary of their query or create a file note entry after the interaction, although both of these approaches place additional effort on either the part of the citizen or on the front-line public servant. Without integration between ICTs and the recordkeeping system, while this type of emergent technology makes it easy to interact with members of the public, it can introduce additional effort for staff to capture the records, which, in turn, may lead to decreased engagement in recordkeeping tasks by staff members.

The second focus group also discussed attitudes towards digital records, with particular reference to staff not appreciating "that if they write the business text message, it has actually become a record" (Rachel, Focus Group 2). Similar to the online chat example raised by the first focus group, this technology makes the creation of records simple for staff, but has implications for recordkeeping, in particular how to integrate with existing recordkeeping systems without becoming burdensome for staff. Also discussed by the focus group attendees was the level of trust that front-line staff have towards ICTs, which can influence whether they save records to the appropriate information repositories. Furthermore, focus group attendees suggested that digital literacy of front-line staff had implications for the capture of digital records in appropriate systems (as observed during the fieldwork in Vignette 5.1).

The third focus group also discussed the influence of OIA legislation on the information management values, attitudes and behaviours of front-line public 
servants within the state sector, suggesting that as a result of the discoverable nature of records captured within recordkeeping systems, fewer records are captured. This scenario, where public servants avoided creating a record, was not observed during the fieldwork, although as discussed the fieldwork participants were aware that details captured in records could be requested by members of the public and participants described the care they took to reflect the interaction in an accurate and formal manner.

\subsubsection{Business process}

The second facet of analysis within the recordkeeping informatics approach is a review of business processes in ways that identify the ongoing use and the adequate capture of recorded information at appropriate points in business processes (Upward et al., 2013, p. 45).

At all three case study organisations, manual involvement from the participants was necessary to capture and manage records, whether this was inputting details, completing the metadata, or categorising the records. At both ERMA New Zealand and Te Puni Kōkiri, participants had the discretion to decide what records to capture within the recordkeeping systems. The focus of this decision was primarily based on what details they or their immediate team might require to complete the relevant piece of work, but there was also awareness from some staff of their responsibilities to capture records from an organisational perspective. Participants explained that if they did not complete the process at the point of capture, for example by completing metadata or providing meaningful file names, then it was unlikely that they would return to do this at a later point.

Inland Revenue call centre staff had little discretion about what records to capture as some of the organisational ICTs they used initiated the capture process. For example, 
when participants answered the phone, details about the call, such as date, time, duration and the IRD number of the member of the public were automatically recorded in the system. In addition to front-line staff having little discretion about what records to capture, business processes and ICT configuration determined where participants captured records. All records relating to interactions with customers were captured within the FIRST system, while other ICTs capturing records by front-line staff had specific pre-determined uses, for example, LOLA, which generated letters based on templates and prepared them for distribution.

The focus group attendees discussed an intention to embed record capture activities as much as possible within business processes. The focus groups discussed how this required a 'ground-up' redesign of business processes and supporting ICTs, where recordkeeping was only one, relatively minor, component. However, this was challenging; as discussed by the focus groups, many existing initiatives tended to be team or workgroup focused solutions, such as annual key performance indicators for recordkeeping set for one or two teams within an organisation, and access to particular ICTs for specific business activities.

The focus group attendees discussed the business process dimension from their perspectives as public servants with responsibilities for recordkeeping across organisations. Their perspectives on the business process dimension reflected challenges within organisations to manage records within hybrid recordkeeping systems, which required ensuring that the recordkeeping systems provided the necessary technological and business functionality and fully supported relevant business processes, reflecting the organisational culture. According to the focus group attendees, IT staff designed and implemented ICTs with little focus on the recordkeeping functionality or recordkeeping behaviours of staff; in many ways, anticipating that the ICTs would provide comprehensive functionality, without 
necessarily focusing on the culture or behaviours of staff interacting with the systems.

The focus group attendees also suggested that organisations and some records managers don't appreciate the entire recordkeeping system within organisations, focusing instead on the EDRMS or the physical filing as the only records repository. The focus group attendees also discussed that technologies change rapidly, and in some organisations may be introduced without awareness from either the IT or recordkeeping staff; organisations struggle to identify solutions and provide the necessary recordkeeping advice and support to staff creating records using emergent ICTs. Similarly, the focus group attendees highlighted challenges in setting organisational policies for recordkeeping; while these may align with the legislative framework, they may not be applicable or meaningful for staff conducting recordkeeping on a daily basis.

\subsubsection{Archival access}

The third facet of analysis within the recordkeeping informatics framework is archival access; one of the most challenging aspects of the recordkeeping agenda, exposing the connections between recordkeeping informatics with political and social concerns (Upward et al., 2013, p. 46).

Some participants were aware of the longevity of records as a result of their use of non-current records. This raised specific concerns about create and capture recordkeeping behaviours, such as identifying which versions of digital records to retain. The prior experiences of front-line ERMA New Zealand participants accessing non-current records influenced their recordkeeping behaviours, for example by ensuring that final versions of records included all signatures and were clearly identified as the final version. 
Participants at Te Puni Kōkiri did not mention accessing non-current records on a regular basis as part of their roles. Some Te Puni Kōkiri participants mentioned they were motivated to manage records to support the broader social environment, and, in particular, acknowledged the concept of collective memory, broader than the agency that they worked in. In a practical sense, this awareness was observed when staff created and captured digital records, which necessitated providing metadata that would remain meaningful beyond the immediate workgroup's activities. Information sharing between Te Puni Kōkiri staff and other government agencies was not observed, although Whetu described a situation where the regional office shared information with a community organisation within the region. This situation demonstrated awareness by staff at the regional office of the broader social environment that information held by the office had within the wider community, particularly with Māori communities. The focus of capturing records was targeted towards the relevant piece of work and sharing records predominantly with staff within the regional office, but there was also awareness from some Te Puni Kōkiri participants of their responsibilities to capture records from an organisational and broader transparent governance perspective. Whetu explained:

\footnotetext{
Because you know for the next generation, who come in after me sort of thing. Because it becomes a historical document that everybody should have access to.

(Whetu, Te Puni Kōkiri)
}

Inland Revenue followed a decentralised approach to recordkeeping where individual business units were responsible for their recordkeeping. All Inland Revenue front-line public servants observed during the fieldwork appreciated the records that they created and captured could be requested as part of an OIA or privacy response, although they were not personally involved in collating the responses. 
The focus group discussions highlighted challenges faced by recordkeeping and information management authorities within the public sector to influence and coordinate organisations' recordkeeping behaviours with social and political concerns. Challenges relating to interoperability of ICTs, including within organisations, placed practical limitations on the extent that records could be deployed beyond one organisation or sometimes beyond one workgroup or one ICT system. Information sharing between agencies was practically limited, for example by bespoke ICTs in place, file formats, and metadata schemas. The focus group attendees also discussed broader societal contexts for recordkeeping, including the underpinning policy settings for recordkeeping within the government. Without clearly articulated goals and foundational principles for recordkeeping, and broader information management aspects across government, the focus group attendees suggested that individual agencies may struggle to be sufficiently motivated to shift towards recordkeeping that acknowledged the social and political concerns beyond individual recordkeeping behaviours, particularly as this shift would require agencies to commit effort and resources to address interoperability challenges.

\subsubsection{Summary of recordkeeping informatics}

Consideration of the three facets of recordkeeping informatics allows for comparative positioning of recordkeeping activities in the three case studies, in relation to each other, while also incorporating the positions discussed within the focus groups.

Participants paid little attention to the relationship between recordkeeping tasks and business processes. Within ERMA New Zealand and Te Puni Kōkiri, participants applied their personal information management values, attitudes and behaviours to recordkeeping tasks; whereas for Inland Revenue participants the analysis to ensure recorded information was captured at appropriate points in business processes had 
already been embedded in ICTs. At the time of the fieldwork, there was little evidence of consideration for the connections between recordkeeping informatics with political and social concerns by front-line public servants participating in this study. However, this awareness was raised by the focus group participants, who discussed the need for broader cross-government approaches to recordkeeping, for example, the focus on information governance incorporating recordkeeping as well as other, broader information management considerations and all-of-government approaches to risk.

\subsection{Personal information management strategies}

As described in the literature review, the personal information management strategies of filing and piling provide a framework to understand how people manage information in the workplace (Malone, 1983); while this framework was developed in a purely physical environment, Henderson (2009) expanded this framework to include a third strategy of structuring and updated descriptions of the strategies to reflect personal information management behaviours in digital contexts. This section explores the organisational contexts for personal information management, based on the strategies of piling, filing and structuring.

\subsubsection{Piling}

Malone (1983, p. 106) defined piling as a personal information management strategy where individual elements (for example, papers and folders) were not necessarily titled, and they were generally not arranged in any particular order. Henderson (2009, p. 133) developed a persona description of a 'piler' in a hybrid, physical and ICT-enabled, environment:

Alex doesn't really file his documents, he just lets them pile up on his desktop until it is full and he dumps them into a folder. Because of this he has a fairly shallow and 
broad file system, with a higher proportion of documents at the top level of his structure. He doesn't really use the tree (since he doesn't really have many folders organised into a hierarchy) and is more likely to search for lost files if he can't find them using chronology.

No 'pilers' as described above were observed during the fieldwork; all three organisations had some measures in place to limit individuals' abilities to pile records, although these measures were more tightly enforced and monitored at Inland Revenue, motivated by the culture of managing private details of members of the public.

The technical environments in place at the three case study organisations encouraged staff to manage records in structured environments, either using the EDRMS, the CRM or the organisation's physical filing system, all of which actively discouraged the concept of 'piling' records. The three organisations employed digital controls to discourage piling digital records, including automated reminders that staff received when sending emails to save the emails in the EDRMS, as well as size restrictions set on Outlook and personal drives. At Inland Revenue, there were few opportunities to store digital records in areas external to the organisational recordkeeping systems and staff members' approaches to 'piling' records, either digital or physical, were actively monitored and followed up.

Inland Revenue participants were not observed using their desktops, personal drive or piling work-related emails; in addition the front-line staff were discouraged from interacting with customers using email, which significantly reduced the volume of emails created or received. USB ports on Inland Revenue computers were disabled, removing the ability for staff to use portable storage devices. 
On the other hand, as previously described by Glenn and as explained by Janet below, some ERMA New Zealand and Te Puni Kōkiri participants did take a piling approach when managing draft or ephemeral records, particularly digital records:

I use my desktop [...] it's like stuff that I'm working on. When it's finished or significant copies, then it goes into the EDRMS. Because it just gets a bit messy for everybody else. (Janet, ERMA New Zealand)

The participants at ERMA New Zealand and Te Puni Kōkiri were also observed saving records to portable storage devices for working out of the office (for example, Vignettes 4.4 and 5.3).

Staff at all three case study organisations were seated at small physical work areas often with a single credenza and a shared bookshelf in an open-plan office. During the observations, participants at ERMA New Zealand and Te Puni Kōkiri stored small amounts of work-in-progress documents on their desks, as well as storing physical reference information, such as books and reference articles on nearby shelves (for example, in Vignette 4.3 Glenn refers to a book on a shelf in his immediate work area). Inland Revenue staff followed strict guidelines on the use and management of paper records, whereby if they completed an interaction with a customer, they sent any physical records received to the records storage and destroyed any physical notes made using confidential bins straight away.

The focus group attendees discussed the piling strategy from their perspectives as managers responsible for organisation-wide approaches to recordkeeping, and thus tended to either dismiss the piling strategy as something out of their control that was difficult to manage, or assumed the piles of physical records were duplicates of records stored elsewhere in the organisational recordkeeping system: 
We have kind of come to an agreement and anybody who hands me something, I'll go "Is this saved on the document management system?" and they will say "Yes". I'll go "Well we don't actually need to keep this". So they have gradually learned that they can keep their own records on their desk, and I'm happy with that. They can keep their own physical filing.

(Jennifer, Focus Group 1)

One challenge discussed by the focus group attendees was their lack of visibility of the extent that staff might be piling digital records. It was possible for them to see piles of physical records on or around staff members desks, and while they may receive reports on the size of staff members Outlook or personal drives, or have organisational limits set for all staff on the maximum size of personal repositories, as in place at ERMA New Zealand and Te Puni Kōkiri, opportunities remained for staff to pile digital records locations without knowledge or oversight by the organisation's recordkeeping staff.

\subsubsection{Filing}

A filing strategy, where people save digital files to folders and store physical records in codafiles, is a more organised approach to managing documented information than the piling strategy. Henderson's (2009, pp. 245-246) description of Linda as a 'filer' provides a rich description of this strategy:

[Linda] customises her workspace to suit herself, putting the documents she uses most within easy reach, and those she rarely uses on her top shelf, and in the awkward-toaccess filing cabinet in the corner under the window. It doesn't bother her if things pile up a bit, but eventually every few months the size of the piles will get too high and she'll have a burst of tidying and put everything away where it belongs. She tries to do pretty much the same thing on her computer, tending to have folders for major projects, topics or responsibilities, with all the files related to that task in the folder. Sometimes if a folder gets too big so she has to scroll a lot, she might consider splitting it. 
Similar to the piling approach, all three organisations had some measures in place to limit individuals' abilities to file records in personal filing structures.

During observations at both ERMA New Zealand and Te Puni Kōkiri, participants faced situations where they intended to save a record to the EDRMS, but were unable to locate an appropriate location within the classification structure, and therefore they saved the items in a location external to the EDRMS, in a personal filing location or did not save them. As outlined in Vignette 4.7, Janet, from ERMA New Zealand, explained that when she was unsure of where to save a particular enquiry, she would save it to her desktop. Similarly, in Vignette 5.1, Whetu was observed trying to save an email to the EDRMS, clicking through the classification hierarchy, but when she was unable to identify an appropriate location to save the item, she sent the email without saving it to the EDRMS, while retaining the sent email in her Outlook. These two examples demonstrate that while the EDRMS at the respective organisations provided a structured approach to recordkeeping, when that structure did not accommodate a specific record, participants employed their personal information management approach to manage the items.

Participants at all three organisations worked with ephemeral information; those documents and data with a relatively short shelf life and that may not be a record as they do not provide evidence of business activity, copies of records, brief notes prior to being transcribed into one of the organisational ICTs, or, as described in Vignette 6.7, the use of digital ephemeral notes by Aroha. Participants were responsible for identifying what was ephemeral, and in the cases of ERMA New Zealand and Te Puni Kōkiri, staff often kept this information on their desks or in credenzas near them. On the other hand, Inland Revenue staff were encouraged to destroy this information regularly; Tracy, from Inland Revenue, explained how she used a notebook to document key discussion points while on the phone with customers; 
where this ephemeral document contained personal details of members of the public, she would use a confidential destruction box to destroy that page at the end of the day.

The focus group attendees had concerns over the extent of any personal information management approach employed by staff, particularly approaches which involved storing records external to the organisation's recordkeeping system. However, the focus group attendees did acknowledge that some staff had personal information management approaches to managing, often digital, records within the workplace. This created challenges when trying to implement hierarchical filing structures for the entire organisation, as staff requested their personal filing structure to be reflected in organisational structures:

Frankly if I hear one more person say they want an intuitive system, I'm going to scream. I say "we can do logical but we can't do intuitive ". You know "Does your colleague agree with your filing system? "No". "Well, who's intuition are we going to use?"

(Anna, Focus Group 2)

Focus groups attendees discussed that some staff considered records that they

created, captured and used during their work to belong to them personally, without consideration of the records from the organisational or broader public sector perspective. As a result, these staff considered that their personal approaches were an improvement on the organisational approaches and therefore, continued to use them, resisting the use of the organisational recordkeeping systems.

\subsubsection{Structuring}

People who adopt a structuring personal information management strategy intensively organise their files, creating deep and meaningful document structures, 
often before there are documents to put in them (Henderson, 2009, p. 239). Henderson (2009, pp. 247-248) described the personal information behaviours of Matthew, a persona representing the structuring strategy:

\begin{abstract}
At work he tends to have a pretty clean desk. He has several organiser boxes on his shelves and paper trays on his desk. He prefers to always have everything in its place so he knows exactly where to find it. Matthew likes to be just as organised on his computer. His desktop is clean - just shortcuts to the one or two items that he accesses all the time, which he changes depending on what he is currently working on. He tries to give every file a descriptive name, sometimes with codes to indicate the year, project or task, and placing it in its proper place in the hierarchy.
\end{abstract}

By their nature, EDRMS support a structuring approach to managing records: metadata is used to categorise records, and a hierarchical classification reflective of the entire organisation is in place. Therefore, at least in relation to digital records, structuring approaches were observed at all three case study organisations.

In relation to physical records, participants, as front-line public servants, were not directly responsible for physical recordkeeping. At all three case study organisations, staff provided physical records to a team who was responsible for managing the physical files. Although at ERMA New Zealand, some staff completed this responsibility themselves, especially where they were the primary users of a physical file and had the file on their desks or nearby. During the observations with Inland Revenue, no participants were observed using physical files to access information; participants sent physical records for filing and were observed accessing digital copies of physical records that had been scanned using the EDSR system. The structuring of physical records, including the digitisation process, was performed by recordkeeping staff and Inland Revenue staff generally had very little physical records on or around their desks, or in surrounding work areas. 
The focus group attendees discussed the structuring strategy with relation to ICTs used by staff within their organisations, suggesting that staff preferred to work within the line of business systems that they used most often to conduct their daily work. Attendees raised the lack of integration between business systems and EDRMS that required staff to manually save records from one system to the other, which as a result discouraged staff from doing so. Therefore, integrating recordkeeping requirements into business systems would ensure that records were structured appropriately, and that they were captured in a meaningful way for staff. Similar to points raised in relation to the piling and filing personal information management strategies, recordkeeping professionals discouraged any structured approaches that were external to the organisation's approved recordkeeping system.

\subsubsection{Summary of personal information management strategies}

The working environment facilitated by organisations significantly influenced frontline staff members' abilities to apply personal information management strategies, in most situations the working environment limited staff members ability to apply any personal approach. The majority of participants were observed working with ICTs and physical files in a structured manner, where digital records had specific metadata applied and both physical and digital records were classified according to an organisational structure. By their nature, EDRMS support this structured approach to managing records. However, it is important to note that while following a structured approach according to Henderson's (2009, pp. 247-248) description, this did not reflect a personal information management approach to recordkeeping; participants did not decide to manage information in a structured way; rather, the ICTs embedded this approach. The ability for participants to make decisions about how to work with the ICTs was limited. Participants were unable to customise or personalise the ICTs, for example by changing the interface to suit how they want to 
create, manage and use the records, applying personal categories or tags to items, by identifying 'favourites', or specifying shortcuts to items they referred to regularly.

The participants at ERMA New Zealand and Te Puni Kōkiri were observed piling or filing records in repositories external to the organisational recordkeeping environment (such as desktops, email, portable storage devices). The participants at Inland Revenue had the least scope to employ personal information management approaches, as they had fewer opportunities to apply discretionary behaviours when creating and capturing records, or to use alternative repositories.

Attendees at all three focus groups discouraged personal information management approaches to recordkeeping, seeking and implementing controls to encourage staff to align their recordkeeping approaches with the organisational policies, procedures and systems. Controls discussed during the focus groups to limit personal information management approaches included both 'soft-system' solutions such as training, inductions, procedures and change management initiatives, as well as 'hard-system' initiatives such as reporting on statistics of ICTs use, placing size limitations on repositories external to the organisational recordkeeping system, and disabling USB ports to restrict use of personal storage devices. There was no discussion by the focus group attendees about how to incorporate personal information management approaches within organisational recordkeeping approaches, for example, the ability for staff to customise and personalise systems.

\subsection{Influencing factors on front-line public servants' decision- making behaviours}

Loyens and Maesschalck (2010, p. 72) outlined four categories of factors that influenced front-line public servants' decision-making behaviour: 
- individual decision-maker characteristics,

- organisational characteristics,

- client attributes, and

- the broader community.

This section examines each of these four categories, reviewing the recordkeeping behaviours observed during the three case studies and the focus groups discussions.

\subsubsection{Individual characteristics}

While difficult to measure the degree of influence of individual characteristics in isolation of other influencing factors, within each of the organisations and as discussed by focus group attendees, participants highlighted ways that their individual characteristics, including their professional backgrounds and previous experiences, and attitudes to ICTs influenced their recordkeeping behaviours.

Participants explained how their profession influenced their recordkeeping behaviours, for example the ERMA New Zealand participants had scientific backgrounds. One of the ERMA New Zealand senior managers further described the influence of employees profession on the recordkeeping behaviours within the organisation by suggesting that as many staff were scientifically trained, they were experienced researchers, and thus preferred to manage records in ways that enabled the records to be retrieved. One Te Puni Kōkiri participant, Pamela, explained that she had begun her working career as a records clerk within the New Zealand public sector and she felt that this previous experience influenced her approach to recordkeeping as it had provided her with a thorough understanding of how the 
broader organisation functioned as well as the importance of access to accurate records.

Where participants responded to information requests or had to address the impact of poor recordkeeping as part of their roles, these experiences also influenced public servants' recordkeeping actions. At ERMA New Zealand, participants explained how their involvement in responding to large and complex legal proceedings and OIA requests led them to be more aware of the need to manage the information at the point of creation or capture, rather doing this retrospectively, acknowledging that this required more time and effort from them during the creation and capture processes. Inland Revenue participants described experiences where colleagues had not sufficiently documented interactions with customers, which led to a lack of details and challenging conversations with members of the public. As a result of these experiences, participants attempted to capture comprehensive and accurate details of all interactions they had with members of the public.

The level of trust that participants had in the recordkeeping systems also influenced how they used the ICTs and managed records within them; S. G Shaw et al. (2013, p. 81) also identified issues with trust as a constraint to using EDRMS. Danielle from ERMA New Zealand explained how she often saved emails into the EDRMS as well as retaining them in her Outlook because she was concerned that she wouldn't be able to locate them again in the EDRMS due to search limitations and previous experiences.

The focus group attendees discussed a number of individual characteristics that they viewed as influencing the recordkeeping behaviours of public servants within their respective organisations, including the profession and age of staff, the nature of the work being conducted, as well as the digital environment of staff at home and the 
ICTs in place at work. Michelle, from the first focus group meeting, reflected that poor recordkeeping behaviours were often attributed to the usability and functionality of ICTs; "I just wondered if it is more the person rather than the tools", suggesting that the influence of individual characteristics on recordkeeping behaviours may be just as important as the ICTs in place. Attendees also suggested that age may be a factor in how staff manage records; the attendees discussed that while older staff may understand the concepts of recordkeeping and the benefit of managing full and accurate records, they may struggle to complete their digital recordkeeping responsibilities due to their digital literacy, preferring instead to print and file records.

There was also consideration amongst focus group attendees that the nature of the work conducted by staff influenced their recordkeeping behaviours, in particular, that structured process work required different recordkeeping behaviours to the recordkeeping behaviours of analysts and advisors, or knowledge workers, where the business processes tended to be less prescriptive. The attendees suggested that where work is process-driven at least some recordkeeping behaviours can be embedded into the business processes and the relevant ICTs.

\subsubsection{Organisational characteristics}

This section outlines how organisational characteristics, including the organisational structure, staff workloads and training provided to staff, influenced the recordkeeping behaviours of participants.

While ERMA New Zealand comprised of a single location, the organisational structure of Te Puni Kōkiri included a head office and ten regional offices. Pamela, who travelled around the country for her role often visiting other regional offices, explained that the recordkeeping procedures in each office were consistent, meaning 
that she could retrieve physical files easily regardless of the office she was visiting as all of the regional offices used the same coding system. However, one participant suggested that while the recordkeeping procedures were similar between head office and the various regional offices, as a result of the different types of records dealt with at the head office, the volume of the records and the limited number of staff, the recordkeeping behaviours differed. Similarly, participants at Inland Revenue had mixed opinions on whether recordkeeping behaviours were similar within a head office and regional offices, although participants suggested that where there were discrepancies between offices, efforts were in place to create alignment as much as possible, recognising the different types of work and records handled at each office.

The case study participants described organisational initiatives designed to influence their recordkeeping behaviours, in particular, the training they had received. Participants explained that the recordkeeping training occurred as part of the implementation of the EDRMS and for new employees. Participants at all three organisations described how the focus of the training was technical; how to use the EDRMS and other systems to capture and manage records. Inland Revenue call centre staff received comprehensive staff inductions and the regular training; in addition, organisational posters were observed around the regional office, reminding staff of their responsibilities handling customer information:

\section{Vignette 8.1: Organisational poster for 'Keeping Our Information Safe'}

There is a poster on a nearby pillar for Integrity Awareness Week 8-12 July, which displays a traffic light image with the text:

Keeping Our Information Safe: 
STOP Am I dealing with customer information?

THINK Are my actions going to keep the customer's information safe?

GO Yes, I'm sure the information I'm responsible for is safe

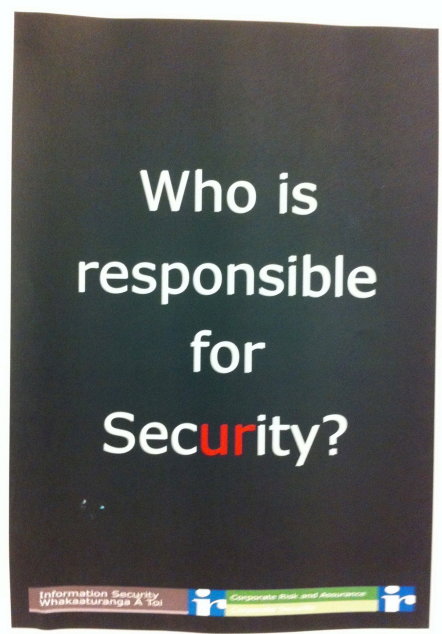

Figure 8.1: Information security poster at Inland Revenue

Each of these messages reinforced the security and privacy responsibilities of call centre staff dealing with personal details of members of the public; the training and change management initiatives at Inland Revenue strongly emphasised these aspects of the legislative framework, rather than staffs' recordkeeping responsibilities, consequently, the participants were unaware of their responsibilities according to the PRA.

ERMA New Zealand participants explained that their workload influenced their ability to perform the recordkeeping responsibilities expected of them; if they were busy, then managing records became a lower priority task. This is similar to the finding by S.G. Shaw et al. (2013) that inconvenience and prioritisation of tasks 
limited the use of the EDRMS. Some ERMA New Zealand participants felt that completing recordkeeping related tasks was a requirement in addition to their primary role and something to do when they were not busy:

I am Science Manager, New Organisms. So in that role, I overview the science that goes into applications and assessing applications. And I manage a small group of advisors. And that takes all my time - there's no time for filing.

(Glenn, ERMA New Zealand)

While not directly related to client attributes, some Te Puni Kōkiri staff were required to work out of the office, and they indicated and demonstrated how this impacted the creation, management, and use of records (for example, in Vignette 5.3). Te Puni Kōkiri participants explained that the lack of reliable internet access in some areas of New Zealand restricted staff member's ability to access the EDRMS and other ICTs, which necessitated the use of portable storage devices, the hard drive on laptops as well as printing records in preparation for offsite meetings.

The focus group attendees also discussed organisational characteristics that influenced the recordkeeping behaviours of staff. At all three meetings, attendees raised the influence of managers and colleagues on individual recordkeeping behaviours:

People can be doing things great, but as soon as they see one person not doing it, then they all start to wander off as well. You know how it is, if it's tolerated that some people don't have to do it, or the Chief Executive doesn't have to do it then, "Is it a thing, is it not a thing, do I have to do this?" So it becomes unclear and then individual practices come into play a lot more.

(Michelle, Focus Group 1)

The role of managers as those responsible for allocating budgets and resources to support recordkeeping was also explored. 
The focus group attendees also suggested that the policies and training provided by the organisation influenced the recordkeeping behaviours, although not always in a positive way. For example, some attendees discussed situations where organisational policies set overarching statements, but they were not sufficiently granular to inform how individuals were required to deal with records on a daily basis. A further organisational characteristic raised by the focus group attendees was the potential impact of the size of the organisation, particularly in relation to security of information. In larger organisations, the attendees suggested that staff were more likely to challenge having open access to records within the larger work unit or across the organisation; whereas, from an organisational perspective, open access to information is a significant benefit of a recordkeeping system.

\subsubsection{Client attributes}

Loyens and Maesschalck (2010, p. 73) suggested that the most varied strand of research concerned the potential influence of the third category, 'client attributes'. This section outlines how communicating complex or personal details with members of the public had some influence on individual recordkeeping behaviours, although participants placed less emphasis on this influencing factor than the other factors.

Where ERMA New Zealand participants communicated complex scientific information to members of the public, they explained that they preferred to interact with members of the public in writing. This approach influenced the recordkeeping behaviours of both the ERMA New Zealand advisor and the citizen, as staff requested the citizen to create a record and the advisor to capture the record of the initial conversation as well as the related email correspondence. Inland Revenue participants also raised concerns about situations where customers misunderstood or misinterpreted messages, and it motivated staff to capture sufficient evidence of conversations to avoid this situation. 
Within Inland Revenue, as discussed previously, significant amounts of personal information about members of the public were created, managed and used, sometimes over long periods of time. Within this agency, there was evidence of a culture where front-line staff followed specific behaviours to protect customer's personal information, beginning with the information creation process:

\footnotetext{
We have to be really careful about notes because it can't contain anything personal in case the director requests information and we've referred to an associate by name - it can get a bit tricky.

(Courtney, Inland Revenue)
}

Privacy was not a significant concern for ERMA New Zealand staff, although there were some specific considerations advisors needed to follow when handling confidential information provided as part of HSNO applications. However, handling confidential information submitted to ERMA New Zealand as part of an application was not observed during the fieldwork or discussed by the participants, suggesting this did not occur often.

There was a little discussion by the focus group attendees on the influence of client attributes on recordkeeping behaviours. In the first focus group, the attendees discussed the influence of clients on recordkeeping behaviours from a different perspective, suggesting that organisations must address the ICTs in place for members of the public to interact with government, particularly as the use of ICTs to engage with government is increasing. Rachel S suggested that members of the public will "get frustrated because they are using certain type of technology that sometimes government department aren't quite up at that level."

\subsubsection{The broader community}


Loyens and Maesschalck (2010, p. 73) explained that factors external to the organisation also impacted on the decision-making of front-line public servants. In an exploration of recordkeeping behaviours, extra-organisational factors identified through analysis of the three cases and the focus group discussions included the legislation and information sharing between government agencies.

As mentioned in relation to organisational training, Inland Revenue staff had significant knowledge on legislation requirements relating to privacy and tax legislations, but little in relation to the PRA. Participants at ERMA New Zealand had some knowledge of the existence of the PRA, but had varying levels of understanding of any specific requirements defined by the legislative framework for recordkeeping. This lack of knowledge of the PRA legislation by participants at all three of the case study agencies does not indicate that staff were not complying with their obligations set out by the legislation. Julie S, the Corporate Services manager at ERMA New Zealand responsible for recordkeeping and information management, explained that the agency looked to embed controls within the EDRMS to support legislative compliance, such as removing the ability for individuals to delete records. The focus for her was to ensure compliance with legislation was as simple as possible for staff.

All three agencies had relationships with other government agencies, requiring staff to work with other public servants. ERMA New Zealand also worked closely with other agencies, in particular, the former Ministry of Agriculture and Forestry, and while the information shared between these agencies was not about individuals, it was potentially sensitive particularly when still in draft form, as described in the following vignette:

\section{Vignette 8.2: Information sharing between agencies}


Janet is on the phone to a Ministry of Agriculture and Forestry staff member and they are updating each other on specific pieces of work. Janet says to the Ministry of Agriculture and Forestry employee that she is willing to send her a confidential email on a not yet publicly released decision on a species of orchids. Janet explains that the document has been released to some Ministry of Agriculture and Forestry staff already, but she specifically asks this person to treat the information carefully.

This sharing of information between ERMA New Zealand and Ministry of Agriculture and Forestry was ad hoc where the individual staff members defined the information sharing conditions based on their personal expectations and their relationships with staff at the receiving agencies. Sharing information between public agencies was also a discussion point raised by front-line staff within Inland Revenue. Brenda from Inland Revenue explained that as front-line staff interacting with members of the public, they may become aware of certain situations or need information from other agencies that they did not have access to. The Inland Revenue information sharing obligations were embedded in formal templates and organisational processes, whereby staff had little opportunity to deviate from these.

The focus group attendees highlighted the role of legislation as well as expectations for all-of-government recordkeeping and information management set by ministers when discussing the influence of the broader community on individual recordkeeping behaviours.

And there is a behavioural shift to happen but that is only at the beginning and there is a long way to go before actually gets the benefits that ministers want. (Henry, Focus Group 3) 
This point was further expanded upon by Henry, when he discussed the role of the legislative framework, suggesting that the policy settings underpinning the legislation were critical as these defined what the role of records and information was within the public sector, its value and how it needed to be open, but also protected. The focus group attendees also discussed the nature of the public sector as an influencing factor on the recordkeeping behaviours of public servants. In general, all attendees agreed that the nature of recordkeeping is different in the public sector to that of the private sector. As discussed in Section 7.3.3, the focus group attendees explained that the public sector, and the associated types of work, roles and personalities, as well as legislative framework, influenced the recordkeeping behaviours. Although, the discussion also questioned how relevant these differences are within the current state sector agencies, suggesting that some values of public servants are not as prevalent as previously. "Is bureaucracy actually separate to what public servants do? This what a public service job requires", Anna asked of the second focus group, arguing that recordkeeping is a core responsibility of public servants, reinforcing the broader social context that front-line public servants recordkeeping occurs within.

\subsubsection{Summary of influencing factors on front-line public servants' decision-making behaviours}

There are numerous influencing factors on the recordkeeping behaviours of individuals, some of which are encouraged and supported by recordkeeping professionals within organisations, such as setting policies, providing training, initiating change management measures, and embedding specific procedures and templates into ICT-enabled business processes. While other influencing factors identified through this analysis may not be traditionally considered part of the recordkeeping environment, such as the professional backgrounds and previous experiences of staff or the workloads of staff. Overall, there was less evidence of 
client attributes influencing recordkeeping behaviours of individuals, suggesting that the create, manage and use recordkeeping behaviours remain consistent regardless of the customers.

\subsection{Recordkeeping and governance}

The literature review identified five significant ways that recordkeeping contributes to governance: recordkeeping supports accountability, supports compliance with legislation, provides evidence of business activities, ensures transparency and protects secure and restricted records (Dikopoulou \& Mihiotis, 2012; Iacovino, 2005; Willis, 2005). This section explores the empirical evidence from the fieldwork, utilising these five significant themes as a framework for discussion and to assist in the identification of governance implications of recordkeeping for individuals, organisations and the broader public sector, while also examining the roles that both legislation and ethics play in the recordkeeping behaviours of front-line public servants.

\subsubsection{Recordkeeping supports accountability}

While there are many definitions and multiple approaches to accountability, for the purposes of this analysis accountability refers to answering for the things one does (Willis, 2005, p. 89).

Accountability as a specific motivating factor for individual recordkeeping behaviours was not discussed by the case study participants, and there was limited evidence that the requirement to demonstrate accountability motivated participants to create or capture records. One participant, Sharon from ERMA New Zealand, explained that she can be concerned about capturing specific types of records or records from specific people or institutions to demonstrate accountability. However, 
as Sharon describes, this approach is ad hoc, depending on judgements that she makes based on the content and context of each record:

It depends, if I send something to someone internal, like Legal, for comment... no, I'd probably file their [response] if it is especially important and sets a precedent and outlines how I should proceed with things I'd file that too. And... it depends on who it is. So if it's internal discussion around things then or management sign-off on a copy... yeah, it depends how important it is. It depends on whether I need to cover my arse or not.

(Sharon, ERMA New Zealand)

None of the case study organisations provided advice or details to staff about identifying specific records that should be kept for accountability purposes, or how to consequently create, manage and use accountability records.

Discussions during the focus groups suggested that attitudes towards recordkeeping for accountability, particularly from a risk perspective, may be different between the private and public sector:

There is a risk bottom line... and I don't think, I mean the public service has risks definitely, but each individual public servant doesn't have that same level of risk as I think you have in private sector, where if you screw up or lose things you are on the line.

(Anna, Focus Group Meeting 2)

Accountability is included as one of the main purposes of the PRA (Public Records Act, 2005). The evidence from the fieldwork suggests that while recordkeeping within the public sector is critical as a means of being able to answer for the things one does and is articulated in the legislation as a core purpose of records and recordkeeping, accountability was not often considered as a factor impacting public servants' recordkeeping behaviours by individuals or by organisations. 


\subsubsection{Recordkeeping supports compliance with legislation}

From an individual perspective, this research found that front-line public servants are not highly motivated by specific pieces of recordkeeping legislation or agency recordkeeping policies, and they may not have detailed knowledge of either. This finding that recordkeeping legislation does not motivate individuals to perform specific recordkeeping behaviours is supported by Lips, Rapson and Hooper (2008, p. 595) who found that the decisions made by New Zealand central government employees when managing email reflected personal email management requirements, rather than an understanding of their legal, democratic or managerial requirements.

The focus groups discussed that although the recordkeeping legislation in New Zealand is principle-based, challenges still exist for agencies to set policies and procedures that staff can understand and follow within the context of their work. There are no responsibilities for individual public servants set out within the legislative framework for recordkeeping or in the state servants' code of conduct, which can make it difficult for public agencies to communicate individual recordkeeping responsibilities and for public servants to apply the legislation in their daily work situations. Conversely, for example, in the United Kingdom, public servants are formally responsible for keeping a record of their activities, as the "Integrity" section of the Civil Service Code states that individuals should keep accurate official records and handle information as openly as possible within the legal framework (Cabinet Office Digital Records and Information Management Team, 2017, pp. 15-16).

\subsubsection{Recordkeeping provides evidence of business activities}


Recordkeeping provides ongoing evidence or proof of a particular activity (Iacovino, 2005, pp. 255-256). As outlined by Archives New Zealand (2016a, p. 4), records serve as evidence of business activity and contribute to the corporate memory of an organisation. For the fieldwork participants, creating and maintaining records as evidence of business activities they were involved in was the primary means that their individual recordkeeping behaviours impacted on the governance of their immediate team, organisation and the public sector. This sentiment was repeated with participants from all three case studies: they created records and captured them in the organisational recordkeeping system to ensure the records were available as evidence of completed activities or decisions made.

Participants also discussed implications if evidence in the form of records were unavailable; for example, Inland Revenue call centre staff speaking to members of the public, attempting to confirm previous statements made by Inland Revenue colleagues. Focus group participants also discussed the need to capture evidence of staff members' activities to ensure continuity of business activities.

The evidence from the fieldwork suggests that providing evidence of business activities through appropriate recordkeeping is considered as a factor impacting public servants' recordkeeping behaviours and generally occurs as part of the regular daily work of front-line public servants. However, in many cases, the creation of records to provide evidence was not a conscious behaviour by participants; part of the daily work of front-line public servants necessitated the creation of records as evidence, for example the decision-making reports at ERMA New Zealand (Vignette 4.3), the capturing of details surrounding Te Puni Kōkiri funding applications (Vignette 5.2) and calls between Inland Revenue front-line staff and members of the public (Vignettes 6.1 and 6.5). 


\subsubsection{Recordkeeping ensures transparency}

While there are many definitions and multiple approaches to transparency, for the purposes of this analysis transparency refers to doing things in a way which is open to appropriate scrutiny (Willis, 2005, p. 89).

As Sharon highlighted, the need for transparency of business activities depended on the nature of the records used:

\footnotetext{
It depends on what it is. If it's a big formal thing like reviewing a standard or revising a standard, then I would tend to track the progress of it because that's quite important. But if it's just a document on a particular issue in a set time I don't think I'd be going through the versions.

(Sharon, ERMA New Zealand)
}

However, as Sharon describes, this approach is dependent on how she judges the need for transparency, based her understanding of the content and context of each record at the point of creation. Transparency was also inferred by Whetu from Te Puni Kōkiri, when she described records captured in the EDRMS as being available to all Te Puni Kōkiri staff, and for future generations. In this way, Whetu acknowledged that records have a role beyond their immediate creation and can be used by others to be able to scrutinise how decisions were made, activities conducted and policies or standards defined.

From an open data perspective, the participants involved in all three case studies were aware of their responsibilities to create and capture records according to the requirements of the OIA and the Privacy Act. Both pieces of legislation influenced the content of records created by participants (for example, Inland Revenue participants did not include any names or details of others when capturing details of interactions with members of the public), and the case study organisations, 
particularly Inland Revenue, provided significant guidance to staff on their responsibilities to create and capture records of interactions with citizens, ensuring information was available if requested. From a cross-government perspective, there is a shift towards open data, as outlined in the 2011 Declaration on Open and Transparent Government, whereby the New Zealand government committed to actively releasing high-value public data (Cabinet Office, 2011). Although, the processes for sharing records between agencies varied from informal and casual to prescribed, formal approaches.

The evidence from the fieldwork suggests that ensuring records are available for others to scrutinise is considered as a factor impacting public servants' recordkeeping behaviours, but in relation to compliance with OIA and Privacy Act legislation, rather than driven by recordkeeping requirements.

\subsubsection{Recordkeeping protects secure and restricted records}

Protecting secure and restricted records was not a topic discussed to a great extent by the fieldwork participants or the focus group attendees, with the exception of Inland Revenue front-line staff. Information privacy and security were not significant concerns for ERMA New Zealand or Te Puni Kōkiri participants due to the nature of information collected, although for ERMA New Zealand there were some specific considerations that advisors needed to follow when handling confidential information provided as part of HSNO applications. On the other hand, Inland Revenue participants created, managed and used significant amounts of personal information about members of the public and this greatly influenced the culture of the Service Delivery functional area, and the entire agency. As well as contributing to the specific organisational culture, protecting personal information did influence recordkeeping behaviours within Inland Revenue, including the 
formats used to create records, and limiting the use of email to communicate with members of the public.

The three case study organisations each had specific guidelines regarding protecting the security and privacy of information; in the most part, these related to upholding the requirements of the Privacy Act, as well as legislation and standards that established specific information and recordkeeping requirements for the agencies, such as the Tax Administration Act for Inland Revenue. The three case studies also had the infrastructure in place to support the creation, management and use of private and secure information according to information handled by the agency classified by the New Zealand Government Security Classification System (Department of Prime Minister and Cabinet, 2014).

The evidence from the fieldwork suggests that protecting secure and restricted records through appropriate recordkeeping is considered as a factor impacting public servants' recordkeeping behaviours for particular records (for example, confidential information provided as part of ERMA New Zealand applications) or organisations (for example, Inland Revenue).

\subsection{Chapter summary}

This chapter shifted the attention from the micro-recordkeeping behaviours observed during the fieldwork to the broader organisational context for recordkeeping and social environment.

The recordkeeping informatics (refer to Section 2.4.2) provides a framework for addressing recordkeeping recognising technological, organisational and societal contexts. While an emerging approach, the focus on organisational culture, business process and archival access underpinned with continuum thinking and metadata 
provide a relevant and thought-provoking extension of records continuum. Within ERMA New Zealand and Te Puni Kōkiri, participants applied their personal information management values, attitudes and behaviours to recordkeeping tasks; whereas for Inland Revenue participants the ability to ensure recorded information was captured at appropriate points in business processes had already been embedded in ICTs. In general, participants from all three case study organisations paid little attention to the relationship between recordkeeping tasks and business processes; similarly, at the time of the fieldwork, participants paid little consideration the connections between recordkeeping informatics and political and social concerns. However, awareness of both of these facets was raised by the focus group participants, who discussed the need for broader cross-government approaches to recordkeeping, for example, the focus on information governance incorporating recordkeeping as well as other, broader information management considerations and all-of-government approaches to risk.

The personal information management strategies of piling, filing and structuring provide useful ways to describe and compare individual's recordkeeping behaviours. However, the extent that any individual employed within the state sector can apply personal information management strategies is limited as demonstrated by the controls implemented by agencies that encourage the use of organisational recordkeeping systems and discourage and restrict storing records in personal information management environments. Controls observed during the fieldwork included 'soft-system' initiatives such as training, inductions, policies and procedures. 'Hard-system' initiatives were also observed, such as reporting on statistics of ICTs use, placing size limitations on repositories external to the organisational recordkeeping system, disabling USB ports to remove the ability to use of personal storage devices and removing individuals' abilities to delete digital records from ICTs. Personal information management strategies do not 
acknowledge the broader use of records beyond the individual. For this reason, this study found personal information management strategies unable to contribute to the development of an understanding the recordkeeping behaviours of New Zealand front-line public servants in ICT-enabled environments.

Factors influencing the decision-making behaviours of front-line public servants are not typically used to describe or interpret recordkeeping behaviours; however, they have shown to provide a useful lens through which to understand recordkeeping behaviours. This chapter explored the ways that individual decision-making characteristics, organisational characteristics, and the broader community influenced the recordkeeping behaviours of front-line public servants in ICT-enabled public agencies. This study did not attempt to measure the impact of these influencing factors; therefore, it is not possible to say that one carried more weight than another. However, the fieldwork identified that client attributes had little influence on the recordkeeping behaviours of the front-line public servants, thus based on the empirical evidence of this study, the recordkeeping behaviours of individuals remained consistent regardless of the individual customer.

The exploration of the influencing factors on public servants' decision-making behaviours as a component of this study leads to the theoretical proposition that factors influencing recordkeeping behaviours of front-line public servants include personal characteristics, organisational characteristics and the broader community; and exclude client attributes. 


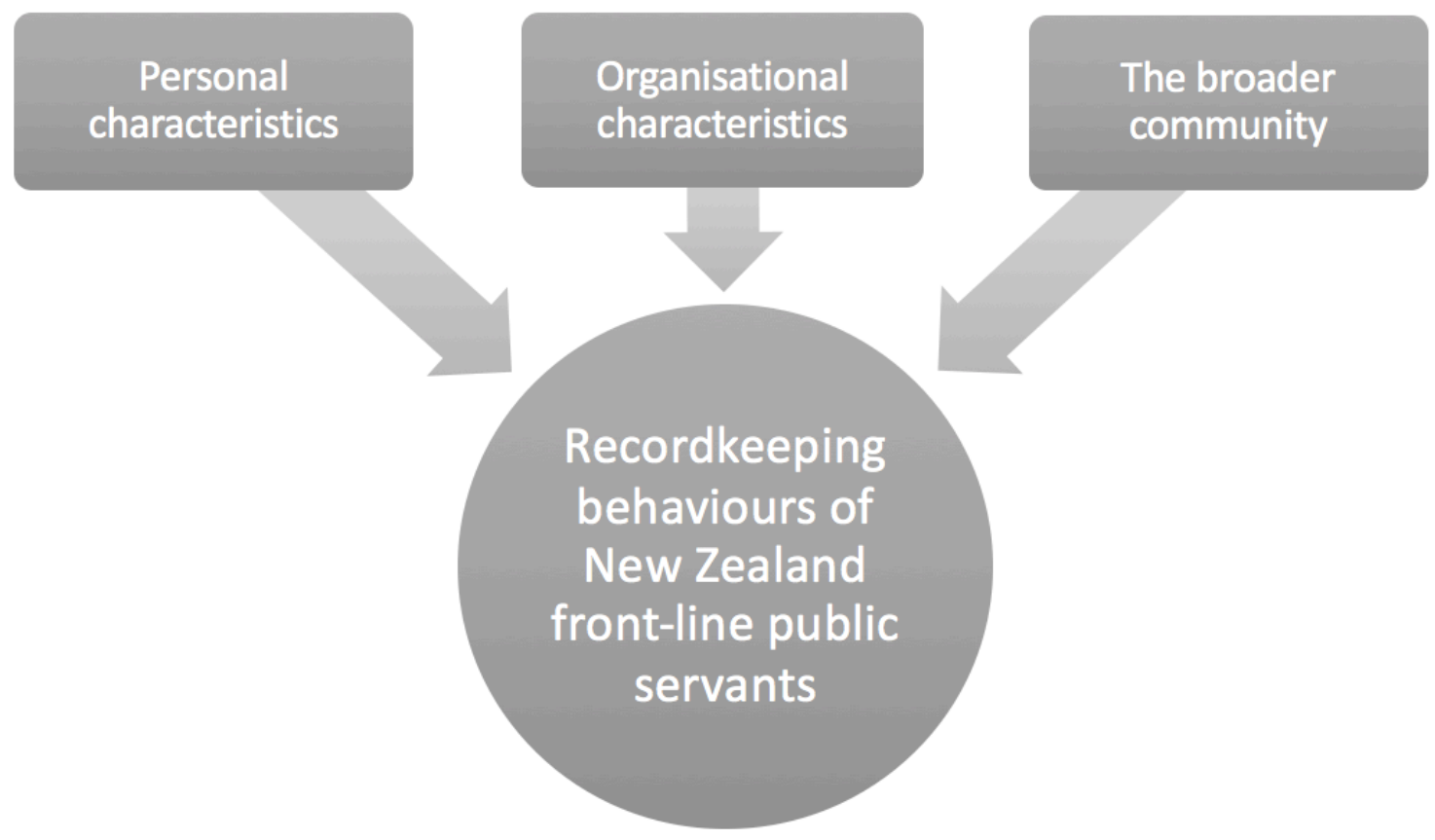

Figure 8.2: Influencing factors on recordkeeping behaviours of New Zealand front-line public servants in ICT-enabled environments

Examples of personal characteristics that influenced recordkeeping behaviours included the professional background, and previous experiences of individuals. Examples of organisational characteristics observed and discussed during the fieldwork concentrated on organisational structure and training provided to staff, which was mostly technical training. The broader community influenced the recordkeeping behaviours observed during the fieldwork, in particular, the nature of the public sector, which provides a specific working environment for public servants.

These findings further culminate in relation to street-level bureaucracy theory, which was developed by Lipsky (1980). Lipsky (1980) described front-line public servants as 'street-level bureaucrats' and argued that the decisions of street-level bureaucrats, the routines they established and the devices they used to cope with 
uncertainties and work pressures effectively become the public policies that they carried out (1980, p. xii). Bovens and Zouridis (2002, p. 180) proposed that streetlevel bureaucrats were increasingly being replaced with a network of screen-level bureaucracies, whereby the decision-making process was routinised and contacts with members of the public always ran through or were in the presence of a computer screen. Gradually, Bovens and Zouridis (2002, p. 181) suggested this would change into a system-level bureaucracy, where system-level bureaucrats had the discretionary power to convert legal frameworks into concrete algorithms, decision trees, and modules. However, recent empirical research argues that there is little evidence that front-line public servants are operating in a system-level bureaucracy, with few wide-spread examples of screen-level bureaucracy (Hansen et al., 2016; Schuppan, 2015).

This research proposes that the influencing factors relating to recordkeeping behaviours impact on the potential applicability of screen- and system-level bureaucracy. At Inland Revenue, where roles and processes are generally prescriptive and staff are employed for their abilities to follow instructions, the ICTs can be used to predetermine particular ways of working, operating in a screen-level bureaucracy manner, with the potential to shift to a system-level bureaucracy; consequently, the creation and capture of records, at least to some extent, can occur as part of standard business processes embedded within ICTs. On the other hand, at ERMA New Zealand and Te Puni Kōkiri, where business activities and the corresponding pieces of documented evidence were less prescriptive and knowledge workers were employed based on their experiences and depth of knowledge in specific areas, there were only some elements of screen-level bureaucracy apparent and limited evidence of system-level bureaucracy. At both ERMA New Zealand and Te Puni Kōkiri the human intervention element was fundamental in all interactions and business activities, particularly as interactions with citizens were unable to be 
reproduced and the steps involved in business activities were difficult to prescribe or predict, requiring extensive and in-depth knowledge, reducing the ability for ICTs to predetermine or prescribe particular way of working. 


\section{Conclusions}

\subsection{Introduction to this chapter}

Recordkeeping is a ubiquitous task within the public sector; public agencies rely on evidence of activities, in the form of public records, to coordinate activities, track resources, establish precedents, monitor performance as well as maintain consistency and continuity. While the public sector would struggle to function without the creation and management of recorded information as evidence, public agencies delegate responsibilities for the creation and management of records to public servants. However, to date, little is known about the recordkeeping behaviours of front-line public servants, particularly in ICT-enabled agencies.

The purpose of this study is to explore the recordkeeping behaviours of New Zealand front-line public servants within ICT-enabled state sector agencies to understand these behaviours and their associated governance implications. Employing a case study approach, which involved collecting data from semistructured interviews, observations, and documentary sources, contributes rich insight into the recordkeeping behaviours of front-line public servants within ICTenabled state sector agencies in New Zealand and resulted in the development of a framework. This framework incorporates the individual characteristics of the decision-maker, the organisational characteristics as well as the broader community as factors that influence the recordkeeping behaviours of front-line public servants. This approach, supported by the second data collection phase, which involved a series of three focus groups, facilitated further understanding of the broader governance implications of the recordkeeping behaviours of front-line public servants, culminating in a framework for interpreting influences on individuals' recordkeeping behaviours. 
This final chapter returns to the research questions posed in Chapter 1. This chapter also discusses the contributions of this study and explores the benefits to academics and the practical implications for practitioners, managers and technical staff. This chapter concludes by identifying limitations of the study and discussing potential areas for future research.

\subsection{Research questions}

This section responds to the three research questions outlined in the introduction to this study.

\subsubsection{What recordkeeping behaviours do New Zealand front-line public servants demonstrate in an ICT-enabled environment?}

Lewellen (2015, p. 27) suggested that recordkeeping actions occurred as close as possible to the moment of creation. However, findings from this study suggest that the creation and capture actions for participants were in part pre-determined by the business processes and ICTs in place, as well as partly the personal motivations for recordkeeping displayed by the participants; did they understand recordkeeping as a core component of their role or did they view recordkeeping as an extra task required of them? In general, the participants at ERMA New Zealand and Te Puni Kōkiri, as knowledge workers, had greater discretion to decide when, how and where to capture records.

The behaviours that participants undertook to use the records captured in the recordkeeping systems also varied between the three agencies. In many ways, these varied behaviours were a direct consequence of the recordkeeping systems prevalent at each agency, which enforced particular ways of working with the records.

However, if the organisations' pre-determined approaches to recordkeeping did not align with participants' expectations or requirements, and if they had the discretion 
to do so, then some participants delayed using the EDRMS until they finalised the record, or looked for ways to overcome system limitations. Where participants delayed using the EDRMS, they retained personal control over the use of and access to the records.

The focus group attendees suggested that the approach whereby public servants retained control over some records had increased as a consequence of the introduction of ICTs to manage records and reflected a general focus by public servants on personal work contributions, as opposed to appreciating the value of the records for the organisation and the broader public sector.

This study also acknowledges that while digital recordkeeping is typically associated with the use of an EDRMS, with records going into and out of the EDRMS, the reality for the participants was that the organisational recordkeeping system was considerably broader than just a single EDRMS, involving multiple ICTs, as well as the traditional paper-based records, engaging with public servants in other parts of the organisation and for some, external to their agency, as well as multiple means of interacting with members of the public, including ICT-enabled, telephone and face-to-face contact. Each of these aspects impacted the resultant recordkeeping behaviours by the individual.

\subsubsection{How can these recordkeeping behaviours be understood?}

In the absence of existing empirical research, this study aimed to develop an understanding of the recordkeeping behaviours of front-line staff within the context of the New Zealand ICT-enabled state sector agencies.

Within ERMA New Zealand and Te Puni Kōkiri, participants applied their personal information management values, attitudes and behaviours to recordkeeping tasks; 
whereas for Inland Revenue participants the ability to ensure recorded information was captured at appropriate points in business processes had already been embedded in ICTs. In general, participants from all three case study organisations paid little attention to the relationship between recordkeeping tasks and business processes; similarly, at the time of the fieldwork, participants paid little consideration the connections between recordkeeping informatics and political and social concerns. Only some participants acknowledged the role of records beyond their immediate team: for the organisation and the broader public sector. The participants were primarily motivated to create and manage records based on their personal need for the record to complete a specific work activity. This finding aligns with Atherton's (1985, p. 49) statement that: “Records are not created to serve the interests of some future archivist or historian, or even to document for posterity some significant decision or operation. They are created and managed to serve immediate operational needs". However, awareness of recordkeeping beyond the individual and their immediate teams, from the perspectives of business processes and archival access was raised by the focus group participants, who discussed the need for broader cross-government approaches to recordkeeping, for example, the focus on information governance incorporating recordkeeping as well as other, broader information management considerations and all-of-government approaches to risk.

While this study identified significant weaknesses in the application of personal information management strategies to understand the recordkeeping behaviours of public servants, the study examined factors influencing front-line public servants' recordkeeping behaviours. In particular, individual decision-maker characteristics, organisational characteristics and the broader community were observed influencing the recordkeeping behaviours of participants. 
Individual decision-maker characteristics identified during the case studies and the focus groups highlighted that the previous experiences of participants impacted how they created and managed records. For example, involvement in responding to large and complex legal proceedings and OIA requests, as well as the impact of incomplete or inaccurate records on staff's ability to complete a task, resulted in participants placing more effort on populating metadata and taking comprehensive notes of interactions.

This study also demonstrated that organisational characteristics influenced the recordkeeping behaviours of New Zealand front-line public servants, including the internal structure of the organisation, staff's workload pressure, and the policies, training and change management provided by the organisation. Challenges arose for participants if policies were not sufficiently granular to inform how individuals needed to deal with records on a daily basis, or where training was focused on technical know-how without supporting details on what records to capture, when, where to save them and why these behaviours were important. The workload allocated by the organisation for specific roles and the impact of managers also influenced the recordkeeping behaviours of participants. Participants were less likely to create and capture records in the recordkeeping systems if they were pressured for time or did not see their managers or senior managers within the organisation actively using the recordkeeping system. In addition, aspects relating to the ICTs influenced the recordkeeping behaviours of the participants, including the trust that individuals had in the system. Within the case studies, observations highlighted the evolving nature of ICTs and that much of the responsibility to address inadequacies or system limitations fell on public servants.

This study also demonstrates that client attributes did not influence the recordkeeping behaviours of New Zealand front-line public servants to the same 
extent as the other influencing factors outlined by Loyens and Maesschalck (2010). Communicating complex or personal details with members of the public as well as conducting face-to-face interactions with people outside of the agency had some influence on individual recordkeeping behaviours, although there was less focus on this influencing factor than the other factors by both the case study participants and the focus group attendees. Overall, there was a little evidence collected during the fieldwork and minimal discussion by the focus group attendees on the influence of client attributes on recordkeeping behaviours, suggesting that the recordkeeping behaviours of front-line public servants are not influenced by the client they are interacting with at any one time; the create, manage and use recordkeeping behaviours remain consistent regardless of the customers.

This study demonstrates that the broader community influenced the recordkeeping behaviours of New Zealand front-line public servants, including legislation and information sharing between government agencies. For example, sharing information while becoming increasingly common within the New Zealand public sector, between individuals within agencies this may be ad hoc based on individuals' personal expectations and their relationships with staff at the receiving agencies; or it can be tightly embedded within business processes and involving multiple monitoring opportunities by management. Either of these approaches has significant effects for the recordkeeping behaviours undertaken by the front-line public servants involved.

To further understand the recordkeeping behaviours of front-line public servants, this study reviewed the empirical findings according to the characteristics of streetlevel, screen-level and system-level bureaucracies proposed by Bovens and Zouridis (2002, p. 180). This study observed participants creating, managing and using records within elements of a 'screen-level bureaucracy'; that is, an environment 
where ICTs have a leading role in interactions between front-line public servants and members of the public. As well as impacting the relationship between government and citizens, from a recordkeeping perspective, the shift to screen-level bureaucracy is particularly applicable for clearly defined processes, whereby the recordkeeping behaviours can be embedded within the ICTs.

\subsubsection{What are the governance implications of the recordkeeping behaviours of New Zealand front-line public servants?}

While governments are reliant on records to function and the legislative framework specifies recordkeeping statements for public agencies, it is the behaviours of public servants interacting with members of the public who ultimately create, manage and use public records.

The literature review identified five significant ways that recordkeeping contributes to governance:

- records support accountability;

- records support compliance with legislation;

- records provide evidence of business activities;

- records ensure transparency; and

- records protect secure and restricted records (Dikopoulou \& Mihiotis, 2012; Iacovino, 2005; Willis, 2005). 
This study examined each of these themes to further understand the governance implications of the recordkeeping behaviours of New Zealand front-line public servants.

Participants in the fieldwork conducted as part of this research did not consider supporting accountability and ensuring transparency as factors that influenced their recordkeeping behaviours. While this may be appropriate as front-line public servants focus on their daily tasks and role specific outcomes; therefore, they may not have considered their role in supporting accountability and ensuring the transparency of decision-making and business activities within the public sector. However, the relationship between these themes and governance were also not demonstrably considered by the organisations themselves or by the focus group participants. None of the case study organisations provided advice or details to staff about how to identify specific records that should be kept for accountability or transparency purposes, and neither the PRA nor Archives New Zealand guidance documentation included transparency as a purpose or benefit of creating and maintaining records.

On the other hand, the two themes of providing evidence of business activities and protecting secure and restricted records did influence the recordkeeping behaviours of the participants. Providing evidence of business activities through appropriate recordkeeping was considered a factor impacting participants' recordkeeping behaviours and occurred as part of participants regular daily work. However, in many cases, the creation of records to provide evidence was not a conscious behaviour by participants as the business processes were designed to create and capture the records, removing personal decision-making as much as possible from the individuals. Protecting secure and restricted records influenced participants' recordkeeping behaviours for particular records (for example, confidential 
information provided as part of ERMA New Zealand applications) and for units within organisations (for example, Child Protection within Inland Revenue). Protecting secure and restricted records impacted how the participants created records, where they captured records, how they used the information contained in the records and how they shared information with members of the public, with public servants in other agencies, and with colleagues internal to the organisation.

With this understanding of how governance themes influence the actual recordkeeping behaviours of front-line public servants in mind, the current governance environment is one where the primary piece of recordkeeping legislation, the PRA, does not align effectively with other pieces of information management legislation or with some agency specific pieces of legislation. This situation occurs within a governance environment where there is an increasing focus for agencies to share information across organisational borders and to look at big data opportunities.

The main responsibilities set out within the PRA are directed at public offices rather than to public servants, yet it is the public servants who create, manage and use the records that the government is reliant upon. The Standards of Integrity and Conduct for state servants in New Zealand (State Services Commission, 2017) states that public servants must treat information with care and use it only for proper purposes. This statement relates most closely to security and privacy concerns and does not explicitly require public servants to understand how to create records, capture and maintain records or to not destroy them. Without allocating responsibilities for individual public servants within the legislative framework for recordkeeping, it can be difficult for public agencies to communicate individual responsibilities for recordkeeping and for public servants to apply the legislation in their daily work situations. This situation was apparent during the fieldwork; case study participants 
and focus group meeting attendees described how they viewed recordkeeping as an expectation for them to complete in addition to the requirements of the role they were employed for.

On this basis, a core implication for governance in relation to recordkeeping is the identification of the requirement to allocate formal responsibility for New Zealand public servants to create and capture records of their activities. Identifying this responsibility could be a component of an update to the legislative framework for recordkeeping or within the Standards of Integrity and Conduct issued by the State Services Commission; regardless this requirement should be set for all public servants and not dependent on the individual agencies to specify.

\subsection{Contributions of this research}

The findings of this study make original contributions to the body of knowledge relating to the recordkeeping behaviours of front-line public servants.

\subsubsection{Academic value of the research}

There are two predominant features of this study that provide academic value and demonstrate a unique contribution to knowledge.

This study developed a framework to understand factors that influence the recordkeeping behaviours of front-line public servants, which incorporates:

- Individual characteristics of the decision-maker: for example, the professional background, the age and previous experiences of individuals, as well as the digital environment of staff at home. 
- Organisational characteristics: for example, the policies and procedures in place, the software, hardware and digital records systems used to create, manage and make accessible the records as well as the physical equivalents.

- Broader community: for example, the nature of the public sector and the legislative framework for recordkeeping and information management.

Secondly, this study reviewed the governance implications of recordkeeping behaviours of New Zealand front-line public servants. This study identified that participants did not consider supporting accountability, ensuring transparency or compliance with recordkeeping legislation as factors that influenced their personal recordkeeping behaviours. On the other hand, providing evidence of business activities through appropriate recordkeeping and protecting secure and restricted records both impacted participants' recordkeeping behaviours. Understanding the recordkeeping behaviours of front-line public servants from a governance perspective provides an empirical understanding of the role that both legislation and ethics play in the recordkeeping behaviours of front-line public servants.

\subsubsection{Practitioner value of the research}

The rich vignettes that describe the reality of front-line public servants' recordkeeping behaviours included in this study provide value to recordkeeping professionals through an informed awareness of the potential implications of the introduction of new ICTs, as well as of the working reality of the changing behaviours of front-line public servants and the changing nature of public records. This study confirms that individuals create and capture records mostly for their immediate needs with little or no understanding of the legislative or organisational policy requirements. In addition, formal responsibility for New Zealand public servants to create and capture records of their activities is not defined within the 
legislative framework for recordkeeping or at a government-wide level. On this basis, a significant challenge for recordkeeping practitioners is how to introduce and encourage an awareness by the front-line public servants of the organisational and public sector benefits for creating, managing and using records, thus ensuring that records receive the necessary focus at a strategic governance level within New Zealand public sector organisations. Furthermore, addressing the lack of formal responsibility for public servants to create and capture records of their activities within the legislative framework would ensure consistency within the public sector and remove the dependency on individual agencies.

This study also provides value to recordkeeping practitioners through the descriptions and analysis of the use of ICTs by front-line public servants as well as the role and nature of recordkeeping within these ICTs. The opportunities to embed recordkeeping functionality into ICTs are broad, as are opportunities to automate the application of the business context to the records created and captured, providing unique opportunities for recordkeeping practitioners to investigate unobtrusive ways to meet organisational and legislative requirements for recordkeeping. The opportunities to introduce mechanisms for staff to personalise and customise the EDRMS interface to suit their preferred ways of working would also further support recordkeeping behaviours of front-line public servants.

\subsection{Limitations of this research}

Every attempt was made to identify and address limitations at the outset of the research, as well as where possible to strengthen and plan the research design and methodology to reduce the impacts of any limitations. However, two fundamental limitations are worthy of discussion. 
Issues associated with identifying and securing cases restricted the number of case studies conducted; involvement from further organisations would have provided additional findings for analysis and comparison, along with different perspectives. Some agencies did not respond to the initial research request, and several organisations declined to participate in the research. Consequently, there was only a small number of agencies interested in participating from which to select the case sites. The involvement of additional participants within the organisations involved, the possibility of follow-up interviews with participants after the observations and extended observation time in the field would have also brought with it additional data for analysis to enrich the case studies further. Interaction with participants during and immediately after the observations was not possible due to the arrangements with the organisations; this would have provided further data and deeper understandings of the observed behaviours. Also, the case study participants volunteered to be involved in the research, potentially introducing bias as these participants may have made themselves available as a result of their knowledge, understanding and interest in recordkeeping.

As a researcher, my background will have inevitably introduced limitations and bias to the interpretations and analysis of the case studies. I have spent over 15 years working and studying in the recordkeeping and information management profession; as such it is inevitable that my cultural and professional background has influenced the overall study.

\subsection{Future research opportunities}

There are many aspects of the recordkeeping behaviours of front-line public servants that this study did not explore due to time, resource and scope limitations. The multidisciplinary and inductive nature of this study introduces many opportunities for future research, including: 
- This research identified three influencing factors, including the personal characteristics of individual staff, the organisational characteristics as well as the broader community; testing the extent of these factors in a comprehensive manner would provide further value to both academics and practitioners.

- Empirical research into cultural differences between Māori and Pākehā approaches to recorded information. While the literature suggests that there are cultural differences between Māori and Pākehā approaches to recordkeeping (Jacobs \& Falconer, 2004; King, 1978; Lai, 2014; McCauley, 2010; Stevenson \& Callaghan, 2008), empirical research in this area is lacking. Benefits from an increased understanding of these differences would have academic and practical implications at both a governmental-wide perspective as well as for individual government agencies.

- Extending the research to investigate recordkeeping behaviours of individuals from other countries, with different recordkeeping legislation and contexts for comparison with those findings from New Zealand would assist in understanding what makes recordkeeping environments unique. In addition, extending the research to incorporate different types of organisations would provide richer perspectives on creating and capturing evidence of agency activities, for example, local government agencies, nondesk bound public servants such as immigration officials, police officers, Department of Conservations rangers. Further investigation into the recordkeeping behaviours of different roles within the public service, for example, managers and senior managers would also further enhance the empirical understandings of New Zealand recordkeeping behaviours, useful in the development of guidelines, setting policy and ICTs design and implementation. 
- Exploring and developing a comparative basis on the individual recordkeeping behaviours of public sector employees and those of private sector employees would provide valuable insights into the motivations for recordkeeping within different legislative environments.

- Developing an analytical lens that incorporates the information culture framework. As Sundqvist and Svärd (2016, p. 14) argued, the question remains whether there is a tangible correlation between information cultures and records management behaviours. While this study identified that aspects of information culture such as information-related competencies and trust in recordkeeping systems did influence to some extent the recordkeeping behaviours of front-line public servants, the objectives of this research were not to evaluate or analyse recordkeeping behaviours and the information culture framework. However, as there is a lack of empirical research exploring the impact of cultural aspects on recordkeeping (Sundqvist \& Svärd, 2016, p. 14), particularly addressing how information is created, captured and preserved, this would be a valuable area to pursue.

- The research conducted for this study was qualitative in nature. Extending this research by incorporating quantitative research methods would assist in confirming and extending the framework for recordkeeping behaviour identified, potentially to develop detailed personas to illustrate particular recordkeeping approaches.

Each of the above points reflects worthy areas for further research, particularly within the current environment where the relationships between public administration, ICTs and recordkeeping continue to merge and introduce new, 
emergent ways of working for front-line public servants interacting with members of the public.

This study highlights the fundamental importance of the recordkeeping behaviours of individuals within the public sector and provides unique insights into how frontline public servants create, manage and use recorded information as evidence. The study provides a framework for interpreting influences on individuals' recordkeeping behaviours and understanding the broader governance implications of these behaviours, but there remains a significant need for researchers to develop and extend studies into this phenomenon. 


\section{Appendix A: Case study participants ${ }^{12}$}

\begin{tabular}{|c|c|c|c|c|c|}
\hline & Gender & Role & Organisation & Location & Involvement \\
\hline Julie S & Female & Manager & $\begin{array}{l}\text { ERMA New } \\
\text { Zealand }\end{array}$ & Head Office & $\begin{array}{l}\text { Interview } \\
\text { only }\end{array}$ \\
\hline Richard & Male & Manager & $\begin{array}{l}\text { ERMA New } \\
\text { Zealand }\end{array}$ & Head Office & $\begin{array}{l}\text { Interview } \\
\text { only }\end{array}$ \\
\hline Danielle & Female & $\begin{array}{l}\text { Advisor/ } \\
\text { Analyst }\end{array}$ & $\begin{array}{l}\text { ERMA New } \\
\text { Zealand }\end{array}$ & Head Office & $\begin{array}{l}\text { Interview \& } \\
\text { observation }\end{array}$ \\
\hline Janet & Female & $\begin{array}{l}\text { Advisor/ } \\
\text { Analyst }\end{array}$ & $\begin{array}{l}\text { ERMA New } \\
\text { Zealand }\end{array}$ & Head Office & $\begin{array}{l}\text { Interview \& } \\
\text { observation }\end{array}$ \\
\hline Leanne & Female & Manager & $\begin{array}{l}\text { ERMA New } \\
\text { Zealand }\end{array}$ & Head Office & $\begin{array}{l}\text { Interview } \\
\text { only }\end{array}$ \\
\hline Glenn & Male & Manager & $\begin{array}{l}\text { ERMA New } \\
\text { Zealand }\end{array}$ & Head Office & $\begin{array}{l}\text { Interview \& } \\
\text { observation }\end{array}$ \\
\hline Kelly & Female & $\begin{array}{l}\text { Advisor/ } \\
\text { Analyst }\end{array}$ & $\begin{array}{l}\text { ERMA New } \\
\text { Zealand }\end{array}$ & Head Office & $\begin{array}{l}\text { Interview \& } \\
\text { observation }\end{array}$ \\
\hline Sharon & Female & $\begin{array}{l}\text { Advisor/ } \\
\text { Analyst }\end{array}$ & $\begin{array}{l}\text { ERMA New } \\
\text { Zealand }\end{array}$ & Head Office & $\begin{array}{l}\text { Interview \& } \\
\text { observation }\end{array}$ \\
\hline Jessica & Female & $\begin{array}{l}\text { Administrato } \\
\mathrm{r}\end{array}$ & $\begin{array}{l}\text { Te Puni } \\
\text { Kōkiri }\end{array}$ & $\begin{array}{l}\text { Regional } \\
\text { Office }\end{array}$ & $\begin{array}{l}\text { Interview \& } \\
\text { observation }\end{array}$ \\
\hline Pamela & Female & $\begin{array}{l}\text { Advisor/ } \\
\text { Analyst }\end{array}$ & $\begin{array}{l}\text { Te Puni } \\
\text { Kōkiri }\end{array}$ & $\begin{array}{l}\text { Regional } \\
\text { Office }\end{array}$ & $\begin{array}{l}\text { Interview \& } \\
\text { observation }\end{array}$ \\
\hline Whetu & Female & $\begin{array}{l}\text { Advisor/ } \\
\text { Analyst }\end{array}$ & $\begin{array}{l}\text { Te Puni } \\
\text { Kōkiri }\end{array}$ & $\begin{array}{l}\text { Regional } \\
\text { Office }\end{array}$ & $\begin{array}{l}\text { Interview \& } \\
\text { observation }\end{array}$ \\
\hline George & Male & Manager & $\begin{array}{l}\text { Te Puni } \\
\text { Kōkiri }\end{array}$ & Head Office & $\begin{array}{l}\text { Interview } \\
\text { only }\end{array}$ \\
\hline Lynn & Female & Record staff & $\begin{array}{l}\text { Inland } \\
\text { Revenue }\end{array}$ & Head Office & $\begin{array}{l}\text { Interview } \\
\text { only }\end{array}$ \\
\hline Courtney & Female & $\begin{array}{l}\text { Advisor/ } \\
\text { Analyst }\end{array}$ & $\begin{array}{l}\text { Inland } \\
\text { Revenue }\end{array}$ & $\begin{array}{l}\text { Regional } \\
\text { Office }\end{array}$ & $\begin{array}{l}\text { Interview \& } \\
\text { observation }\end{array}$ \\
\hline
\end{tabular}

12 Pseudonyms have been used for all fieldwork participants to protect their identities. 


\begin{tabular}{|c|l|l|l|l|l|}
\hline Deanna & Female & $\begin{array}{l}\text { Advisor/ } \\
\text { Analyst }\end{array}$ & $\begin{array}{l}\text { Inland } \\
\text { Revenue }\end{array}$ & $\begin{array}{l}\text { Regional } \\
\text { Office }\end{array}$ & $\begin{array}{l}\text { Interview \& } \\
\text { observation }\end{array}$ \\
\hline Brenda & Female & $\begin{array}{l}\text { Advisor/ } \\
\text { Analyst }\end{array}$ & $\begin{array}{l}\text { Inland } \\
\text { Revenue }\end{array}$ & $\begin{array}{l}\text { Regional } \\
\text { Office }\end{array}$ & $\begin{array}{l}\text { Interview \& } \\
\text { observation }\end{array}$ \\
\hline Aroha & Female & $\begin{array}{l}\text { Advisor/ } \\
\text { Analyst }\end{array}$ & $\begin{array}{l}\text { Inland } \\
\text { Revenue }\end{array}$ & $\begin{array}{l}\text { Regional } \\
\text { Office }\end{array}$ & $\begin{array}{l}\text { Interview \& } \\
\text { observation }\end{array}$ \\
\hline Tracy & Female & $\begin{array}{l}\text { Advisor/ } \\
\text { Analyst }\end{array}$ & $\begin{array}{l}\text { Inland } \\
\text { Revenue }\end{array}$ & $\begin{array}{l}\text { Regional } \\
\text { Office }\end{array}$ & $\begin{array}{l}\text { Interview \& } \\
\text { observation }\end{array}$ \\
\hline Laura & Female & $\begin{array}{l}\text { Advisor/ } \\
\text { Analyst }\end{array}$ & $\begin{array}{l}\text { Inland } \\
\text { Revenue }\end{array}$ & $\begin{array}{l}\text { Regional } \\
\text { Office }\end{array}$ & $\begin{array}{l}\text { Interview \& } \\
\text { observation }\end{array}$ \\
\hline Karen $\mathbf{Y}$ & Female & $\begin{array}{l}\text { Advisor/ } \\
\text { Analyst }\end{array}$ & $\begin{array}{l}\text { Inland } \\
\text { Revenue }\end{array}$ & $\begin{array}{l}\text { Regional } \\
\text { Office }\end{array}$ & $\begin{array}{l}\text { Interview \& } \\
\text { observation }\end{array}$ \\
\hline Rachel K & Female & $\begin{array}{l}\text { Advisor/ } \\
\text { Analyst }\end{array}$ & $\begin{array}{l}\text { Inland } \\
\text { Revenue }\end{array}$ & $\begin{array}{l}\text { Regional } \\
\text { Office }\end{array}$ & $\begin{array}{l}\text { Interview \& } \\
\text { observation }\end{array}$ \\
\hline Dawn & Female & $\begin{array}{l}\text { Advisor/ } \\
\text { Analyst }\end{array}$ & $\begin{array}{l}\text { Inland } \\
\text { Revenue }\end{array}$ & $\begin{array}{l}\text { Regional } \\
\text { Office }\end{array}$ & $\begin{array}{l}\text { Interview \& } \\
\text { observation }\end{array}$ \\
\hline
\end{tabular}




\title{
Appendix B: Case study interview questions
}

\author{
Interview Discussion Topics - The Recordkeeping Behaviours of New Zealand Government \\ Employees
}

\section{Research: Anita Rapson, School of Government, Victoria University of Wellington}

The interviews and observations are intended to collect data necessary to develop the ethnographic study. The interviews are semi-structured discussions.

The following are the topics covered in each of the interviews:

- Role and responsibility of participants

- Role of information (physical and digital) in participant's daily work

- Information management practices - creating, saving, finding, modifying, managing versions and retaining information

- Management of physical and digital records

- Management of information based on the value of that information - archival, working and ephemeral

- Knowledge of information related legislation and organisational policies 


\section{Appendix C: Codes}

\begin{tabular}{|c|c|}
\hline $\begin{array}{l}\text { Behaviours - } \\
\text { Recordkeeping }\end{array}$ & $\begin{array}{l}\text { Defining records } \\
\text { Deleting } \\
\text { Naming } \\
\text { Printing } \\
\text { Retention } \\
\text { Scanning } \\
\text { Searching } \\
\text { Sharing } \\
\text { Versioning }\end{array}$ \\
\hline $\begin{array}{l}\text { Decision-making } \\
\text { influencing factors }\end{array}$ & $\begin{array}{l}\text { Individual characteristics } \\
\text { Organisational characteristics } \\
\text { Client attributes } \\
\text { Broader community }\end{array}$ \\
\hline Governance & $\begin{array}{l}\text { Ensure transparency } \\
\text { Protecting secure and restricted records } \\
\text { Provide evidence of business activities } \\
\text { Support accountability } \\
\text { Support compliance with legislation }\end{array}$ \\
\hline Information status & $\begin{array}{l}\text { Archived } \\
\text { Ephemeral } \\
\text { Working }\end{array}$ \\
\hline $\begin{array}{l}\text { Legislative } \\
\text { Framework }\end{array}$ & $\begin{array}{l}\text { Official Information Act } \\
\text { Privacy Act } \\
\text { Public Records Act }\end{array}$ \\
\hline $\begin{array}{c}\text { Personal information } \\
\text { management }\end{array}$ & $\begin{array}{l}\text { Piling } \\
\text { Filing } \\
\text { Structuring }\end{array}$ \\
\hline Records continuum & $\begin{array}{l}\text { Create } \\
\text { Capture } \\
\text { Organise } \\
\text { Pluralise }\end{array}$ \\
\hline $\begin{array}{l}\text { Recordkeeping } \\
\text { informatics }\end{array}$ & $\begin{array}{l}\text { Organisational culture } \\
\text { Business process } \\
\text { Archival access }\end{array}$ \\
\hline
\end{tabular}




\section{Appendix D: Focus group participants ${ }^{13}$}

Focus Group Meeting 1:

\begin{tabular}{|c|c|c|c|}
\hline & Gender & Role & Type of Organisation \\
\hline Edith & Female & $\begin{array}{l}\text { Records Manager - } \\
\text { sole charge }\end{array}$ & $\begin{array}{l}\text { Public Service } \\
\text { Department }\end{array}$ \\
\hline Heather & Female & Records Manager & Consultant \\
\hline Janice & Female & $\begin{array}{l}\text { Records Manager - } \\
\text { sole charge }\end{array}$ & Crown Entity \\
\hline Jennifer & Female & $\begin{array}{l}\text { Records Manager - } \\
\text { sole charge }\end{array}$ & Crown Entity \\
\hline Karen C & Female & Records Manager & $\begin{array}{l}\text { Public Service } \\
\text { Department }\end{array}$ \\
\hline Mark & Male & $\begin{array}{l}\text { Advisor at } \\
\text { recordkeeping and } \\
\text { information } \\
\text { management } \\
\text { authority }\end{array}$ & $\begin{array}{l}\text { Public Service } \\
\text { Department }\end{array}$ \\
\hline Michelle & Female & Records Manager & Consultant \\
\hline Thomas & Female & $\begin{array}{l}\text { Records Team } \\
\text { Member }\end{array}$ & Crown Entity \\
\hline
\end{tabular}

${ }^{13}$ Pseudonyms have been used for all fieldwork participants to protect their identities. 
Focus Group Meeting 2:

\begin{tabular}{|c|c|c|c|}
\hline & Gender & Role & Type of Organisation \\
\hline Anna & Female & Lecturer & $\begin{array}{l}\text { Tertiary education } \\
\text { institution }\end{array}$ \\
\hline Ed & Male & Lecturer & $\begin{array}{l}\text { Tertiary education } \\
\text { institutions }\end{array}$ \\
\hline Gail & Female & Lecturer & $\begin{array}{l}\text { Tertiary education } \\
\text { institutions }\end{array}$ \\
\hline Helen & Male & $\begin{array}{l}\text { Advisor at } \\
\text { recordkeeping and } \\
\text { information } \\
\text { management } \\
\text { authority }\end{array}$ & $\begin{array}{l}\text { Public Service } \\
\text { Department }\end{array}$ \\
\hline Henry & Male & $\begin{array}{l}\text { Advisor at } \\
\text { recordkeeping and } \\
\text { information } \\
\text { management } \\
\text { authority }\end{array}$ & $\begin{array}{l}\text { Public Service } \\
\text { Department }\end{array}$ \\
\hline Rachel S & Female & $\begin{array}{l}\text { Advisor at } \\
\text { recordkeeping and } \\
\text { information } \\
\text { management } \\
\text { authority }\end{array}$ & $\begin{array}{l}\text { Public Service } \\
\text { Department }\end{array}$ \\
\hline Ryan & Male & $\begin{array}{l}\text { Advisor at } \\
\text { recordkeeping and } \\
\text { information } \\
\text { management } \\
\text { authority }\end{array}$ & $\begin{array}{l}\text { Public Service } \\
\text { Department }\end{array}$ \\
\hline
\end{tabular}


Focus Group Meeting 3:

\begin{tabular}{|c|c|c|c|}
\hline & Gender & Role & Type of Organisation \\
\hline Julie U & Female & Records Manager & $\begin{array}{l}\text { Public Service } \\
\text { Department }\end{array}$ \\
\hline Kirsten & Female & Records Manager & Consultant \\
\hline Perry & Male & Records Manager & $\begin{array}{l}\text { Public Service } \\
\text { Department }\end{array}$ \\
\hline Philip & Male & $\begin{array}{l}\text { Records Team } \\
\text { Member }\end{array}$ & $\begin{array}{l}\text { Non-public Service } \\
\text { Department }\end{array}$ \\
\hline Portia & Female & Records Manager & $\begin{array}{l}\text { Public Service } \\
\text { Department }\end{array}$ \\
\hline Rebecca & Female & $\begin{array}{l}\text { Records Manager - } \\
\text { sole charge }\end{array}$ & Charity organisation \\
\hline Sadie & Female & Records Manager & $\begin{array}{l}\text { Non-public Service } \\
\text { Department }\end{array}$ \\
\hline Scott & Male & Records Manager & $\begin{array}{l}\text { State Owned } \\
\text { Enterprise }\end{array}$ \\
\hline
\end{tabular}




\section{Appendix E: Discussion topics discussed at focus group 1}

- ERMA New Zealand: Managing records in both physical and digital recordkeeping systems introduced complexities for staff.

- Te Puni Kōkiri: Staff followed ad hoc and individual approaches to using recordkeeping tools.

- Inland Revenue: The culture of the organisation prioritised privacy and information security. 


\section{Appendix F: Organisations contacted for focus group 2}

\begin{tabular}{|c|c|c|}
\hline Organisations & Website & Purpose \\
\hline $\begin{array}{l}\text { Archives and Records } \\
\text { Association of New } \\
\text { Zealand (ARANZ) }\end{array}$ & www.aranz.org.nz & $\begin{array}{l}\text { ARANZ is an incorporated society, } \\
\text { established in 1976, with the aim of } \\
\text { promoting the understanding and } \\
\text { importance of records and archives in New } \\
\text { Zealand. }\end{array}$ \\
\hline $\begin{array}{l}\text { Archives New Zealand, } \\
\text { Department of Internal } \\
\text { Affairs }\end{array}$ & www.archives.govt.nz & $\begin{array}{l}\text { Archives New Zealand, Te Rua Mahara o } \\
\text { te Kāwanatanga is the official guardian of } \\
\text { New Zealand's public archives. }\end{array}$ \\
\hline $\begin{array}{l}\text { Government Chief } \\
\text { Privacy Officer } \\
\text { (GCPO), Department of } \\
\text { Internal Affairs }\end{array}$ & $\begin{array}{l}\text { www.ict.govt.nz/gover } \\
\text { nance-and- } \\
\text { leadership/the-gcio- } \\
\text { team/government- } \\
\text { chief-privacy-officer }\end{array}$ & $\begin{array}{l}\text { The GCPO is responsible for four main } \\
\text { functions: } \\
\text { - providing leadership by setting the } \\
\text { vision for privacy across government, } \\
\text { - building capability, } \\
\text { - } \quad \text { assurance, and } \\
\text { engaging with the Office of the Privacy } \\
\text { Commissioner, and citizens. }\end{array}$ \\
\hline $\begin{array}{l}\text { Government } \\
\text { Information Services, } \\
\text { Department of Internal } \\
\text { Affairs }\end{array}$ & $\begin{array}{l}\text { www.ict.govt.nz/gover } \\
\text { nance-and- } \\
\text { leadership/the-gcio- } \\
\text { team/government- } \\
\text { information-services }\end{array}$ & $\begin{array}{l}\text { Government Information Services leads } \\
\text { delivery of government information } \\
\text { online, through www.govt.nz, web } \\
\text { presences such as data.govt.nz and } \\
\text { jobs.govt.nz, the provision of advice about } \\
\text { Web standards and domain integrity, and } \\
\text { other initiatives to improve the quality of } \\
\text { government's digital presence. }\end{array}$ \\
\hline $\begin{array}{l}\text { Office of the } \\
\text { Ombudsman }\end{array}$ & $\begin{array}{l}\text { www.ombudsman.parl } \\
\text { iament.nz }\end{array}$ & $\begin{array}{l}\text { The Ombudsman helps the community in } \\
\text { its dealings with government agencies, } \\
\text { including handle complaints against } \\
\text { government agencies and undertake } \\
\text { investigations and inspections. }\end{array}$ \\
\hline $\begin{array}{l}\text { Open Government } \\
\text { Data New Zealand }\end{array}$ & $\begin{array}{l}\text { www.data.govt.nz/tool } \\
\text { kit/open-data-in-new- } \\
\text { zealand/open-data-nz }\end{array}$ & $\begin{array}{l}\text { Open Data NZ's work includes: } \\
\text { working with government agencies to } \\
\text { support, inform, troubleshoot and advise } \\
\text { on their release of open government data, } \\
\text { and working with users of open } \\
\text { government data to understand and } \\
\text { represent their data needs, troubleshoot } \\
\text { and connect them with relevant } \\
\text { government contacts. }\end{array}$ \\
\hline
\end{tabular}




\begin{tabular}{|c|c|c|}
\hline $\begin{array}{l}\text { Records and } \\
\text { Information } \\
\text { Management } \\
\text { Professionals } \\
\text { Australasia }\end{array}$ & www.rimpa.com.au & $\begin{array}{l}\text { RIM Professionals Australasia offers a } \\
\text { wide range of activities supporting the } \\
\text { profession of records and information } \\
\text { management. }\end{array}$ \\
\hline $\begin{array}{l}\text { The Open Polytechnic } \\
\text { of New Zealand }\end{array}$ & $\begin{array}{l}\text { www.openpolytechnic. } \\
\text { ac.nz }\end{array}$ & $\begin{array}{l}\text { The Open Polytechnic of New Zealand } \\
\text { offer a variety of information, library and } \\
\text { record-keeping programmes ranging from } \\
\text { the introductory level through to higher- } \\
\text { level qualifications which will support a } \\
\text { career in these fields. }\end{array}$ \\
\hline $\begin{array}{l}\text { Victoria University of } \\
\text { Wellington }\end{array}$ & www.victoria.ac.nz & $\begin{array}{l}\text { The School of Information Management- } \\
\text { in Victoria Business School - offers an } \\
\text { interdisciplinary approach to managing } \\
\text { technology, information and people. }\end{array}$ \\
\hline
\end{tabular}




\section{Appendix G: Slide deck for focus groups 2 and 3}

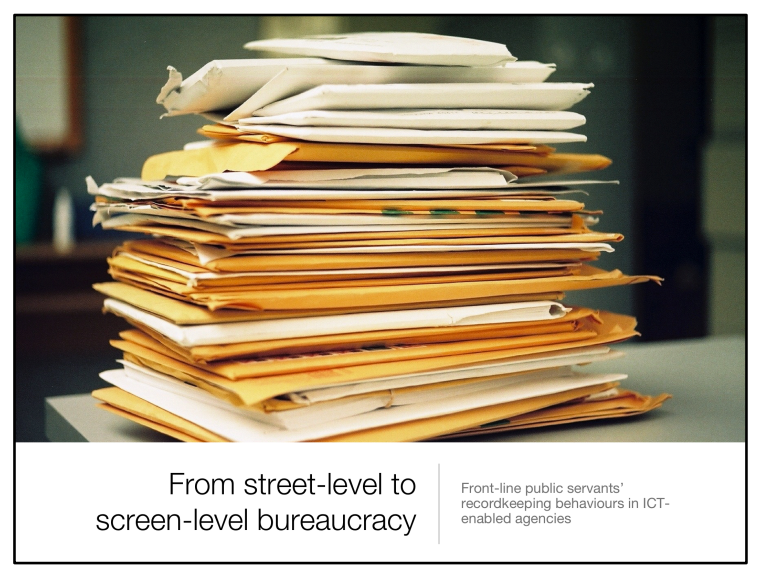

Slide 1

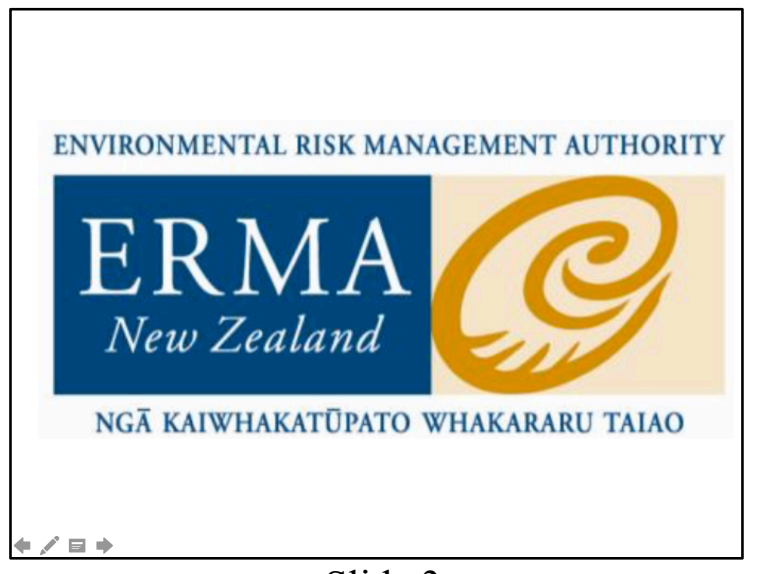

Slide 3

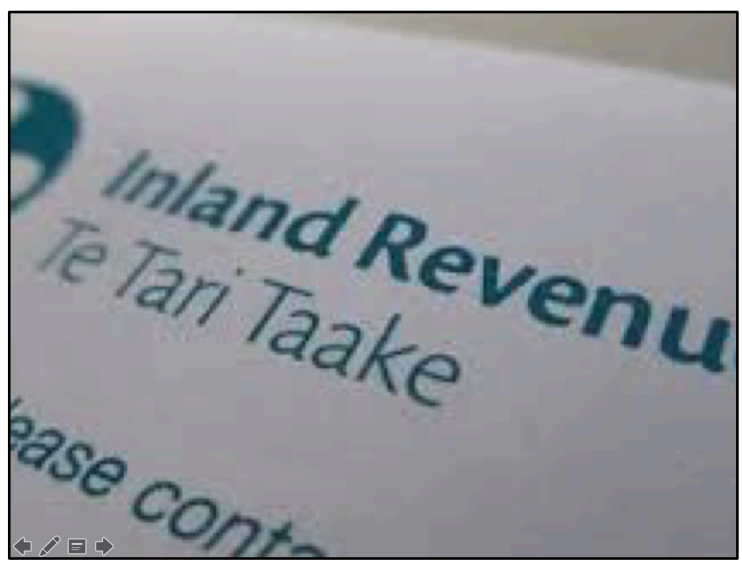

Slide 5

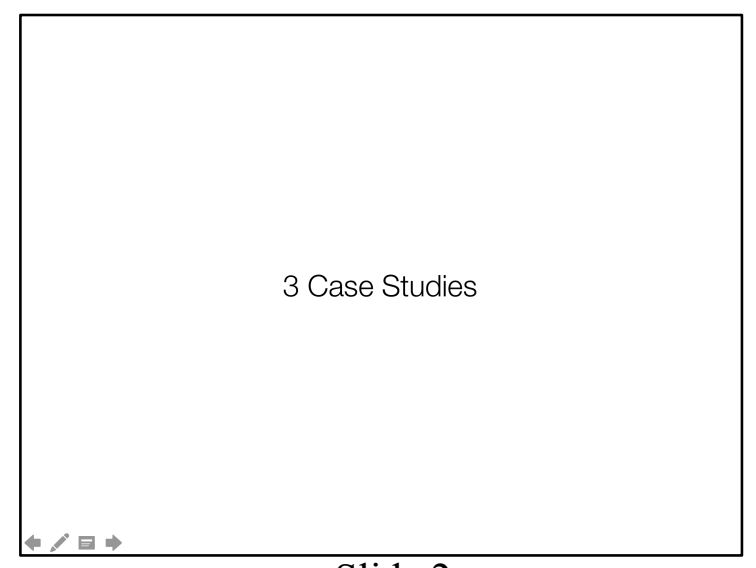

Slide 2

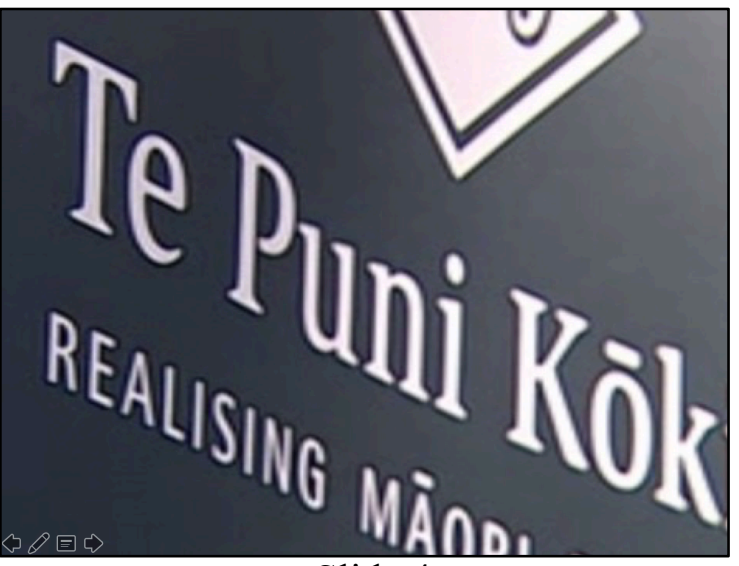

Slide 4

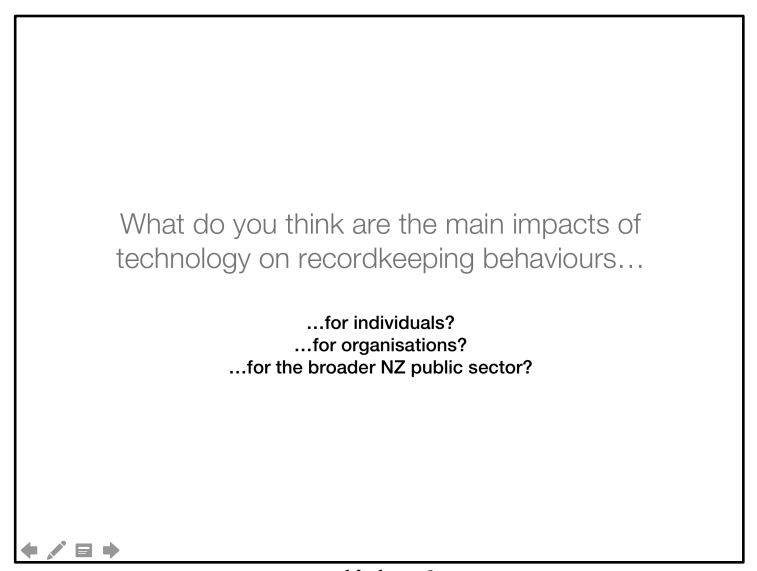

Slide 6 


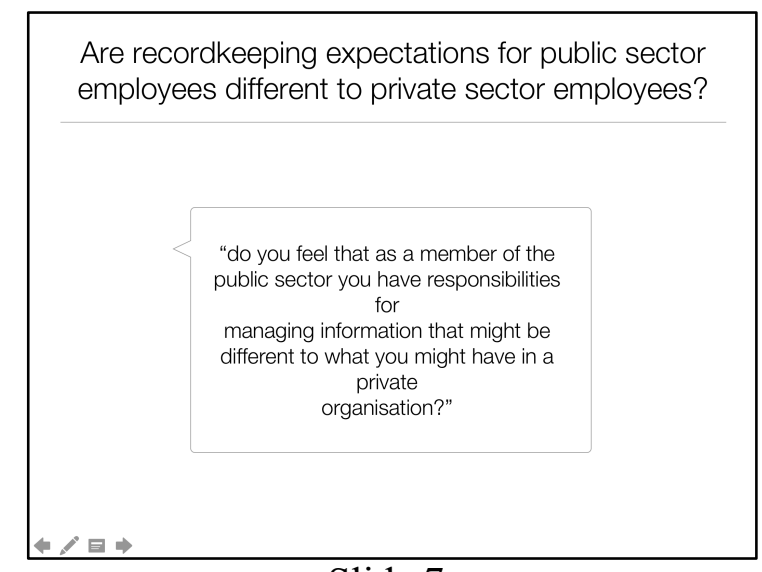

Slide 7

The Role of the Legislative Framework

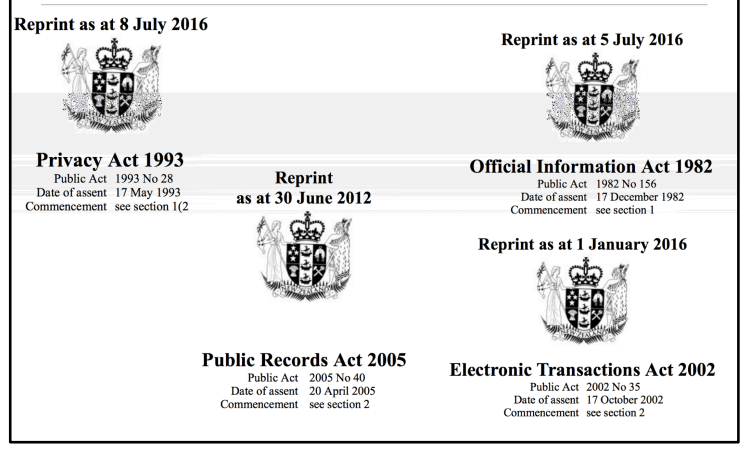

Slide 9

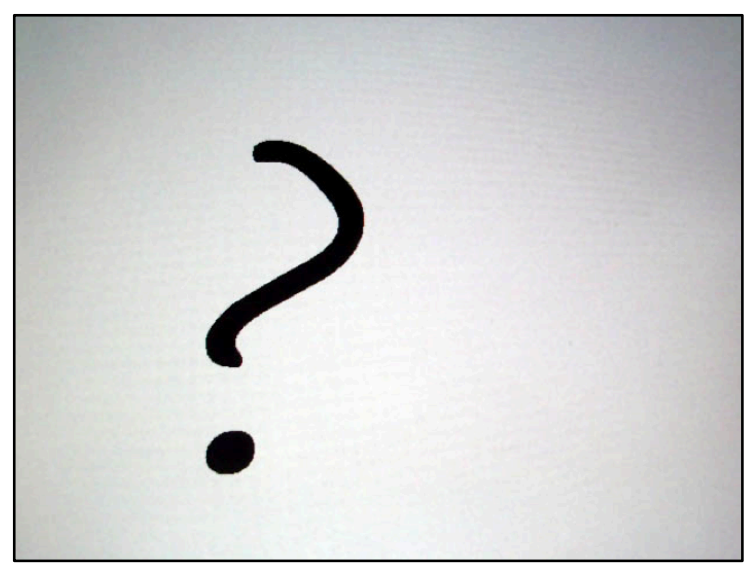

Slide 11
Are recordkeeping expectations for public sector employees different to private sector employees?

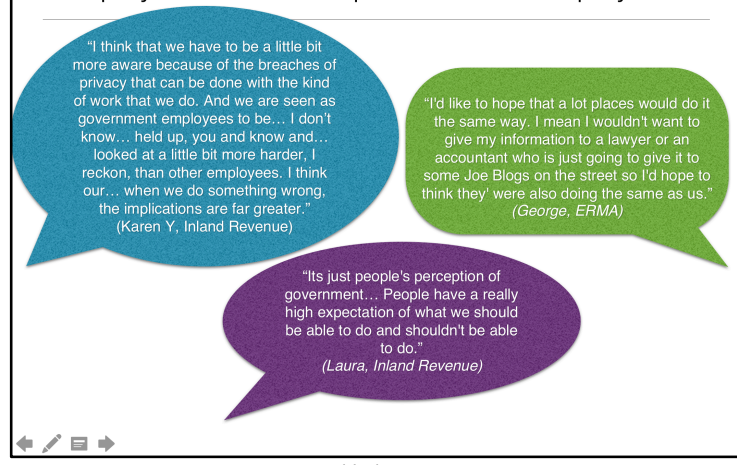

\section{Slide 8}

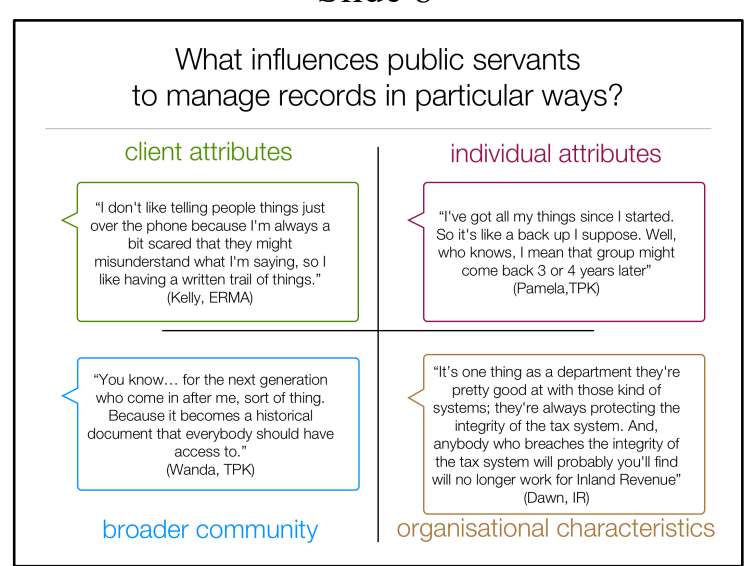

Slide 10 


\section{References}

Abrams, D., Baecker, R., \& Chignell, M. (1998). Information archiving with bookmarks: Personal web space construction and organization. In Proceedings of the SIGCHI Conference on Human Factors in Computing Systems (pp. 41-48). Los Angeles, CA: ACM Press. https://doi.org/10.1145/274644.274651

Adu, K. K., \& Ngulube, P. (2017). Key threats and challenges to the preservation of digital records of public institutions in Ghana. Information, Communication \& Society, 20(8), 1127-1145. https://doi.org/10.1080/1369118X.2016.1218527

Allan, S. A. (2015). Review of government digital records (Independent report). London, England: Cabinet Office. Retrieved from https://www.gov.uk/government/publications/government-digital-records-andarchives-review-by-sir-alex-allan

Andersen, J. (2015). Genre theory in information studies.

Anderson, C., \& Robey, D. (2017). Affordance potency: Explaining the actualization of technology affordances. Information and Organization, 27(2), 100-115. https://doi.org/10.1016/j.infoandorg.2017.03.002

Archives New Zealand. (2015). State of government recordkeeping and Public Records Act 2005 audits 2014/15. Wellington, New Zealand: Archives New Zealand. Retrieved from http://archives.govt.nz/sites/default/files/report__state_of_government_recordkeeping_and_public_records_act_2005_audits_201415_pdf_final.pdf

Archives New Zealand. (2016a). Information and records management standard (A mandatory standard issued under Section 27 of the Public Records Act 2005 by the Chief Archivist No. 16/S1). Wellington, New Zealand: Archives New Zealand. Retrieved from http://records.archives.govt.nz/assets/Guidance-new-standard/16-S1Information-and-records-management-standard-Pdf.pdf 
Archives New Zealand. (2016b, July). Metadata for information and records. Retrieved 14 June 2017, from http://records.archives.govt.nz/resources-and-guides/metadata-forinformation-and-records/

Atherton, J. (1985). From life cycle to continuum. Some thoughts on the records management - archives relationship. Archivaria, 21(Winter), 43-51.

Baarspul, H. C., \& Wilderom, C. P. M. (2011). Do Employees Behave Differently In PublicVs Private-Sector Organizations? Public Management Review, 13(7), 967-1002. https://doi.org/10.1080/14719037.2011.589614

Bälter, O. (2000). Keystroke level analysis of e-mail message organization. Presented at the Conference on Human Factors in Computing Systems (CHI 2000).

Barreau, D. (1995). Context as a factor in personal information management systems. Journal of the American Society for Information Science, 46(5), 327-339.

Barreau, D. (2008). The persistence of behavior and form in the organization of personal information. Journal of the American Society for Information Science and Technology, 59(2), 307-317.

Barreau, D., \& Nardi, B. (1995). Finding and reminding: file organization from the desktop. SIGCHI Bulletin, 27(3).

Barry, R. (2010). Opinion piece - electronic records: now and then. Records Management Journal, 20(2), 157-171. https://doi.org/10.1108/09565691011064304

Bearman, D. (1994). Electronic evidence: Strategies for managing records in contemporary organizations. Pittsburgh, PA: Archives and Museum Informatics.

Benbasat, I., Goldstein, D. K., \& Mead, M. (1987). The case research strategy in studies of information systems. MIS Quarterly, 11(3), 369. https://doi.org/10.2307/248684

Bjerregaard, T., \& Klitmoller, A. (2010). Frontline Problem Solvers: The Structuring of Frontline Service Work. International Journal of Public Administration, 33(8-9), 421-430. https://doi.org/10.1080/01900692.2010.483351

Blaikie, N. (2007). Approaches to social enquiry (Vol. 2). Cambridge: Polity Press. 
Boardman, R., \& Sasse, M. A. (2004). Stuff goes into the computer and doesn't come out: a cross-tool study of personal information management. Presented at the Conference on Human Factors in Computing Systems (CHI 2004).

Boll, K. (2015). Deciding on tax evasion - front line discretion and constraints. Journal of Organizational Ethnography, 4(2), 193-207. https://doi.org/10.1108/JOE-07-20140018

Bovens, M., \& Zouridis, S. (2002). From street-level to system-level bureaucracies: how information and communication technology is transforming administrative discretion and constitutional control. Public Administration Review, 62(2), 174-184.

Buffat, A. (2015). Street-level bureaucracy and e-government. Public Management Review, 17(1), 149-161. https://doi.org/10.1080/14719037.2013.771699

Cabinet Office. (2011). Open government (Cabinet Minute of Decision No. CAB Min (11) 29/12). Wellington, New Zealand: Cabinet Office. Retrieved from https://www.ict.govt.nz/assets/Uploads/Documents/CAB-Min-8-August-2011.pdf

Cabinet Office Digital Records and Information Management Team. (2017). Better information for better government (Policy paper). London, England: Cabinet Office. Retrieved from https://www.gov.uk/government/publications/better-information-forbetter-government

Carroll, B. W., \& Siegel, D. (1999). Service in the field: The world of front-line public servants. Montreal, Canada: McGill-Queen's University Press.

Cavaye, A. L. M. (1996). Case study research: A multi-faceted research approach for IS. Information Systems Journal, 6(3), 227-242.

CCH New Zealand Limited. (2013). New Zealand Master Tax Guide (2013th ed.). New Zealand: CCH New Zealand Limited. Retrieved from http://books.google.co.nz/books?id=aZkwAAAAQBAJ\&pg=PA32\&lpg=PA32\&dq=i nland + revenue + service + delivery\&source $=$ bl\&ots $=$ Xd10eO87Pz\&sig $=J d N B a S q V s k s B$ ilelUB5XHcZkAXo\&hl=en\&sa=X\&ei=_6ZeVIuFOePdmAWp_IHICw\&ved=0CEY Q6AEwBzgU\#v=onepage \&q=inland $\% 20$ revenue $\% 20$ service $\% 20$ delivery $\& \mathrm{f}=$ false 
Childs, S., McLeod, J., \& Hardiman, R. (2011). Accelerating positive change in electronic records management: headline findings from a major research project. Archives and Manuscripts, 39(2), 65-93.

Choo, C. W., Bergeron, P., Detlor, B., \& Heaton, L. (2008). Information culture and information use: An exploratory study of three organizations. Journal of the American Society for Information Science and Technology, 59(5), 792-804. https://doi.org/10.1002/asi.20797

Contract and Commercial Law Act (2017). Retrieved from http://www.legislation.govt.nz

Cook, T. (1994). Electronic records, paper minds: the revolution in information management and archives in the post-custodial and post-modernist era. Archives and Manuscripts, $22(2), 300-328$.

Cook, T. (1997). What is past is prologue: a history of archival ideas since 1898, and the future paradigm shift. Archivaria, 43, 17-63.

Cooper, D. (2016). House proud: an ethnography of the BC Gay and Lesbian Archives. Archival Science, 16(3), 261-288. https://doi.org/10.1007/s10502-015-9250-8

Cooper, J., Lewis, R., \& Urquhart, C. (2004). Using participant or non-participant observation to explain information behaviour. Participant observation, Nonparticipant observation, Information behaviour, Hospital pharmacists, Older people. Information Research: An International Electronic Journal, 9(4), 184.

Copyright Act (1994). Retrieved from http://www.legislation.govt.nz/

Creswell, J. (2003). Research design: Qualitative, quantitative, and mixed methods approaches. Thousand Oaks, CA: Sage Publications.

Creswell, J. (2009). Research design: Qualitative, quantitative, and mixed methods approaches (2nd ed.). Thousand Oaks, CA: Sage Publications.

Creswell, J. (2014). Research design: Qualitative, quantitative, and mixed methods approaches (4th ed.). Thousand Oaks, CA: Sage Publications.

Cullen, R., \& Reilly, P. (2008). Information Privacy and Trust in Government: A CitizenBased Perspective from New Zealand. Journal of Information Technology \& Politics, 4(3), 61-80. https://doi.org/10.1080/19331680801915066 
Cumming, K. (2010). Ways of seeing: contextualising the continuum. Records Management Journal, 20(1), 41-52.

Curry, A., \& Moore, C. (2003). Assessing information culture - an exploratory model. International Journal of Information Management, 23(2), 91-110. https://doi.org/10.1016/S0268-4012(02)00102-0

Davis, F. D. (1986). A technology acceptance model for empirically testing new end-user information systems: theory and results. Massachusetts Institute of Technology, Sloan School of Management, Massachusetts. Retrieved from http://hdl.handle.net/1721.1/15192

deLeon, L. (2003). On acting responsibly in a disorderly world: individual ethics and administrative responsibility. In B. G. Peters \& J. Pierre (Eds.), Handbook of Public Administration. London: Sage Publications. Retrieved from internal-pdf://deLeon, L. (2003). On acting responsibly in a disorderly world individual ethics and administrative responsibility-2349494016/deLeon, L. (2003). On acting responsibly in a disorderly world individual ethics and administrative responsibility.pdf

Department of Internal Affairs. (2005). Document management in Te Puni Kōkiri - the tool. Wellington, New Zealand: Department of Internal Affairs. Retrieved from http://archive.ict.govt.nz/plone/archive/resources/networks/tpk-doc-manage200408/chapter3.html

Department of Prime Minister and Cabinet. (2014, December 18). New Zealand government security classification system. Retrieved 24 May 2017, from https://protectivesecurity.govt.nz/home/information-security-managementprotocol/new-zealand-government-security-classification-system/

Dikopoulou, A., \& Mihiotis, A. (2012). The contribution of records management to good governance. The TQM Journal, 24(2), 123-141. https://doi.org/10.1108/17542731211215071

Douglas, J. (2010). The identification, development and application of information culture in the Western Australian public sector. Edith Cowan University, Perth, Australia. Retrieved from http://ro.ecu.edu.au/theses/1882 
Environmental Risk Management Authority. (2008). Code of conduct. Environmental Risk Management Authority.

Environmental Risk Management Authority. (2009). Records management policy.

Environmental Risk Management Authority.

Environmental Risk Management Authority. (2010). Environmental Risk Management Authority Statement of Intent for the year 2010/2011 (Statement of Intent) (p. 50).

Wellington: Environmental Risk Management Authority. Retrieved from http://www.epa.govt.nz/Publications/ERMA-NZ-Statement-of-Intent\%2020102011.pdf

Environmental Risk Management Authority. (2011). Who we are. Retrieved 18 March 2011, from http://www.ermanz.govt.nz/about-us/who-we-are/Pages/default.aspx

Evans, J., Reed, B., Linger, H., Goss, S., Holmes, D., Drobik, J., ... Henbest, S. (2014).

Winds of change: A recordkeeping informatics approach to information management needs in data-driven research environments. Records Management Journal, 24(3), 205-223. https://doi.org/10.1108/RMJ-01-2014-0006

Findlay, C. (2013). People, records and power: What archives can learn from WikiLeaks. Archives and Manuscripts, 41(1), 7-22. https://doi.org/10.1080/01576895.2013.779926

Fontana, A., \& Frey, J. H. (2005). The interview: from neutral stance to political involvement. In N. K. Denzin \& Y. S. Lincoln (Eds.), The Sage Handbook of Qualitative Research (Vol. Third, pp. 695-727). Thousand Oaks: Sage Publications.

Foscarini, F. (2013). Record as social action: understanding organizational records through the lens of genre theory. Information Research: An International Electronic Journal, 18(3). Retrieved from http://www.informationr.net/ir/183/colis/paperC08.html\#.Wyr-Vy-B1PM

Furnham, A. (2005). The psychology of behaviour at work: the individual in the organization (2nd ed). Hove [England]; New York: Psychology Press.

Gibson, A., Crothers, C., Smith, P., Aguirre, A., \& Bell, A. (2012). Online engagement with government: Insights from the World Internet Project New Zealand. Auckland, New 
Zealand: Institute of Culture, Discourse \& Communication, AUT University. Retrieved from www.wipnz.aut.ac.nz

Gibson, A., Miller, M., Smith, P., Bell, A., \& Crothers, C. (2013). The Internet in New Zealand 2013. Auckland, New Zealand: Institute of Culture, Discourse \& Communication, AUT University. Retrieved from www.wipnz.aut.ac.nz

Gillespie, A., \& Michelson, M. R. (2011). Participant observation and the political scientist: Possibilities, priorities, and practicalities. PS: Political Science \& Politics, 44(02), 261-265. https://doi.org/10.1017/S1049096511000096

Ginmen, M. (1987). Information culture and business performance. In IATUL Proceedings (Vol. 17). Purdue University. Retrieved from https://docs.lib.purdue.edu/iatul/1987/papers/15/

Gracy, K. (2004). Documenting communities of practice: making the case for archival ethnography. Archival Science, 4, 335-365.

Gregory, R. (1982). Understanding public bureaucracy. Public Sector, 4(2/3), 3-12.

Gwizdka, J. (2004). E-mail task management styles: the cleaners and the keepers. Presented at the Conference on Human Factors in Computing Systems (CHI 2004).

Hamel, J., Dufour, S., \& Fortin, D. (1993). Case study methods. Newbury Park, CA: Sage Publications.

Hammersley, M., \& Atkinson, P. (2007). Ethnography: Principles in practice (3rd ed.). London, England: Routledge.

Hansen, H.-T., Lundberg, K., \& Syltevik, L. J. (2016). Digitalization, street-level bureaucracy and welfare users' experiences. Social Policy \& Administration, 1-24. https://doi.org/10.1111/spol.12283

Hardgrave, B. C., \& Johnson, R. A. (2003). Toward an information systems development acceptance model: The case of object-oriented systems development. IEEE Transactions on Engineering Management, 50(3), 322-336. https://doi.org/10.1109/TEM.2003.817293

Harries, S. (2009). Managing records, making knowledge and good governance. Records Management Journal, 19(1), 16-25. 
Henderson, S. (2009). How do people manage their documents? An empirical investigation into personal document management practices among knowledge workers $(\mathrm{PhD}$ Thesis). University of Auckland, Department of Information Systems and Operations Management, Auckland, New Zealand.

Herring, E. P. (1936). Public Administration and the Public Interest. New York: McGrawHill Book Company, Inc.

Huvila, I., Eriksen, J., Häusner, E.-M., \& Jansson, I.-M. (2014). Continuum thinking and the contexts of personal information management. Information Research: An International Electronic Journal, 19(1). Retrieved from http://www.informationr.net/ir/19-1/paper604.html\#.W4R8Ai2B2b

Iacovino, L. (2005). Recordkeeping and juridical governance. In S. McKemmish, M. Piggott, B. Reed, \& F. Upward (Eds.), Archives: Recordkeeping in Society (pp. 255-276). Wagga Wagga: Centre for Information Studies, Charles Sturt University.

Inland Revenue. (2009a). Appraisal report. Wellington, New Zealand: Inland Revenue. Retrieved from http://www.archway.archives.govt.nz/ViewEntity.do?code=DA418 Inland Revenue. (2009b). Briefing for the Incoming Minister of Revenue - 2008. Retrieved from http://www.ird.govt.nz/aboutir/reports/briefing/briefing-2008/bim-08/bim-2008admin.html

Inland Revenue. (2011). Compulsory deductions from bank accounts (No. SPS 11/04). Wellington, New Zealand: Inland Revenue. Retrieved from http://www.ird.govt.nz/technical-tax/standard-practice/returns-debt/sps-11-04compulsory-deductions-from-bank-accounts.html

Inland Revenue. (2012). Application of discretion in section 81(1B) of the Tax Administration Act 1994 - the secrecy provisions (No. SPS 11/07). Wellington, New Zealand: Inland Revenue. Retrieved from http://www.ird.govt.nz/technical-tax/standardpractice/general/sps-1107-application-of-discretion.html Inland Revenue. (2013). Inland Revenue Annual Report 2013 (Annual Report) (p. 147). Wellington, New Zealand: Inland Revenue. Retrieved from http://www.ird.govt.nz/aboutir/reports/annual-report/annual-report-2013/ 
Inland Revenue. (2014a). Briefing for the Incoming Minister of Revenue - 2014:

Administration opportunities. Retrieved from

http://www.ird.govt.nz/aboutir/reports/briefing/briefing-2014/bim-14/bim-2014-

administration-opportunities.html

Inland Revenue. (2014b). Inland Revenue Annual Report 2014 (Annual Report) (p. 136).

Wellington, New Zealand: Inland Revenue. Retrieved from

http://www.ird.govt.nz/resources/3/8/382b8962-6441-4e0c-81bd-

e2b9e2961bfa/annual-report-2014.pdf

Inland Revenue. (2014c). Inland Revenue Statement of Intent 2014-2018 (Statement of

Intent) (p. 37). Wellington, New Zealand: Inland Revenue. Retrieved from

http://www.ird.govt.nz/resources/4/f/4febb0e0-f764-4017-bcfc-

c5bb4eeaf6bf/soi2014.pdf

Inland Revenue. (2014d, March 17). Understanding Compliance. Retrieved 9 November 2014, from http:/www.ird.govt.nz/taxagents/compliance/understanding/

International Council on Archives. (2008). Principles and functional requirements for records in electronic office environments - module 2: Guidelines and functional requirements for electronic records management systems. Paris, France: International Council on Archives. Retrieved from http://www.ica.org/en/node/38970

Isa, A. M. (2009). Records management and the accountability of governance ( $\mathrm{PhD}$ Thesis). University of Glasgow, Glasgow, Scotland. Retrieved from http://theses.gla.ac.uk/1421/1/2009matisaphd.pdf

ISO. (2001). ISO 15489-1:2001 records management. Geneva, Switzerland: ISO.

Jacobs, T., \& Falconer, S. (2004). Ka Mua, Ka Muri; walking backwards into the future.

Paths towards managing Māori Information in Archives. Archifacts, October, 1-19.

Jorna, F., \& Wagenaar, P. (2007). The 'iron cage' strengthened? Discretion and digital discipline. Public Administration, 85(1), 189-214.

Joseph, P. (2010). EDRMS search behaviour: implications for records management principles and practices ( $\mathrm{PhD}$ Thesis). The University of Western Australia, Perth, Australia. Retrieved from http://research- 
repository.uwa.edu.au/en/publications/edrms-search-behaviour-implications-forrecords-management-principles-and-practices(cd09efd9-d97c-4f5e-bf26fc4f19a27a22).html

Kallberg, M. (2013). Issues with contact centres - as a new interface between public organisations and citizens. Records Management Journal, 23(2), 90-103. https://doi.org/10.1108/RMJ-01-2013-0002

Kargbo, J. A. (2010). The connection between good governance and record keeping: the Sierra Leone experience. Journal of Society of Archivists, 30(2), 249-260.

Katsonis, M., \& Sullivan, H. (2014). The 21st century public servant. Public Administration Today, 37(January / March), 16-18.

Keiser, L. R. (2010). Understanding street-level bureaucrats' decision making: determining eligibility in the Social Security Disability program. Public Administration Review, March/April, 247-257.

King, M. (1978). Tihe mauri ora: Aspects of maoritanga. Wellington, New Zealand: Methuen New Zealand Ltd.

Lai, J. C. (2014). Indigenous cultural heritage and intellectual property rights: Learning from the New Zealand experience? New York, NY: Springer.

Lea French, R., \& Williamson, K. (2016). The information practices of welfare workers: Conceptualising and modelling information bricolage. Journal of Documentation, 72(4), 737-754. https://doi.org/10.1108/JDOC-08-2015-0100

Lee, A. (2014). Keynote address. Presented at the 18th Pacific Asia Conference on Information Systems (PACIS 2014), Chengdu, Sichuan, China.

Leidner, D. E. (1988). Understanding information culture: Integrating knowledge management systems into organizations. INSEAD.

Lewellen, M. J. (2015). The impact of the perceived value of records on the use of electronic recordkeeping systems ( $\mathrm{PhD}$ Thesis). Victoria University of Wellington, Wellington, New Zealand.

Librett, M., \& Perrone, D. (2010). Apples and oranges: ethnography and the IRB. Qualitative Research, 10(6), 729-747. https://doi.org/10.1177/1468794110380548 
Lips, M., Eppel, E. A., Sim, D., Barlow, L., \& Lofgren, K. (2014). Kiwis Managing their Online Identity Information (Interim Report - Survey Findings) (p. 118). Wellington, New Zealand: Victoria University of Wellington. Retrieved from http://www.victoria.ac.nz/sog/researchcentres/egovt/research-projects/research2011/KOI_Interim_Report_19March2014v2.pdf

Lips, M., O’Neill, R., \& Eppel, E. (2009). Improving information sharing for effective social outcomes. Retrieved from http://researcharchive.vuw.ac.nz/handle/10063/1581

Lips, M., Rapson, A., \& Hooper, T. (2008). E-mail records management in 21st century New Zealand government. Wellington, New Zealand: Victoria University of Wellington. Retrieved from http://hdl.handle.net/10063/1592

Lips, M., Taylor, J. A., \& Organ, J. (2009). Managing Citizen Identity Information in EGovernment Service Relationships in the UK: The emergence of a Surveillance State or a Service State? Public Management Review, 11(6), 833-856. https://doi.org/10.1080/14719030903318988

Lipsky, M. (1980). Street-level bureaucracy: dilemmas of the individual in public services. New York: Russell Sage Foundation.

Lomas, E. (2013). An autoethnography exploring the engagement of records management through a computer mediated communication focused co-operative inquiry $(\mathrm{PhD}$ Thesis). Northumbria University, Northumbria.

Loyens, K., \& Maesschalck, J. (2010). Toward a theoretical framework for ethical decision making of street-level bureaucracy: existing models reconsidered. Administration and Society, 42(1), 66-100.

Lundberg, K. G., \& Syltevik, L. J. (2016). Everyday interaction at the front-line: The case of the Norwegian all-in-one bureaucracy. Journal of Organizational Ethnography, 5(2), 152-166. https://doi.org/10.1108/JOE-12-2015-0026

Luxton, J. (2008). The Ministry of Maori Development - Te Puni Kōkiri. Te Oranga o te Iwi Maori: a study of Maori economic and social progress (No. Working Paper 4) (p. 20). New Zealand Business Roundtable. Retrieved from http://www.nzbr.org.nz/site/nzbr/files/publications/te_puni_kokiri.pdf 
Mackay, W. E. (1988). Diversity in the use of electronic mail: A preliminary inquiry. $A C M$ Transactions on Information Systems, 6(4), 380-397.

https://doi.org/10.1145/58566.58567

Malone, T. W. (1983). How do people organize their desks? Implications for the design of office information systems. ACM Transactions on Office Information Systems, 1(1), 99-112.

McCauley, D. (2010). Tauranga City Libraries: Meeting the needs of local Iwi? Biculturalism and Maori cultural intellectual property. New Zealand Library \& Information Management Journal, 51(4), 248-268.

McDonald, J. (1995). Managing records in the modern office: taming the wild frontier. Archivaria, 39.

McKemmish, S. (1997). Yesterday, today and tomorrow: a continuum of responsibility. Presented at the Records Management Association of Australia 14th National Convention. Retrieved from http://infotech.monash.edu/research/groups/rcrg/publications/recordscontinuumsmckp2.html

McKemmish, S. (2001). Placing records continuum theory and practice. Archives and Museum Informatics, 1(4), 333-359.

Meehan, A. J. (1986). Record-keeping practices in the policing of juveniles. Journal of Contemporary Ethnography, 15(1), 70-102. https://doi.org/10.1177/0098303986015001003

Meijer, A. J. (2001). Electronic records management and public accountability: Beyond an instrumental approach. The Information Society, 17, 259-270.

Meijer, A. J. (2003). Transparent government: Parliamentary and legal accountability in an information age. Information Polity, 8, 67-78.

Meijer, A. J., \& Homburg, V. M. F. (2008). Introduction: Zooming in and zooming out on electronic government. International Journal of Public Administration, 31(7), 707710. https://doi.org/10.1080/01900690701690700 
Meijer, A. J., \& Torenvlied, R. (2016). Social media and the new organization of government communications: An empirical analysis of twitter usage by the Dutch police. The American Review of Public Administration, 46(2), 143-161. https://doi.org/10.1177/0275074014551381

Miles, M. B., \& Huberman, A. M. (1994). An expanded sourcebook: qualitative data analysis (Vol. 2). Thousand Oaks: Sage Publications.

Ministry of Justice. (2012). Directory of official information 2013: Listings $S$ to $U$. Wellington, New Zealand: Ministry of Justice.

Ministry of Māori Development Act (1991). Retrieved from http://www.legislation.govt.nz/ Moran, J. (2017). Born digital in New Zealand: Report of survey results. Wellington, New Zealand: Alexander Turnbull Library, National Library of New Zealand. Retrieved from https://natlib.govt.nz/librarians/reports-and-research/born-digital-in-new-zealand

Moss, M. S., Endicott-Popovsky, B., \& Dupuis, M. J. (2018). Is digital different?: how information creation, capture, preservation and discovery are being transformed. Retrieved from https://doi.org/10.29085/9781783302376

Mosweu, O., Bwalya, K., \& Mutshewa, A. (2016). Examining factors affecting the adoption and usage of document workflow management system (DWMS) using the UTAUT model: Case of Botswana. Records Management Journal, 26(1), 38-67. https://doi.org/10.1108/RMJ-03-2015-0012

Myers, M. D. (2009). Qualitative research in business and management. Thousand Oaks: Sage Publications.

New Zealand Government Chief Information Officer. (2016a). Government ICT strategy 2015. Retrieved 3 August 2016, from https://www.ict.govt.nz/strategy-and-actionplan/strategy/

New Zealand Government Chief Information Officer. (2016b). New Zealand data and information management principles. Retrieved 25 August 2016, from https://www.ict.govt.nz/guidance-and-resources/open-government/new-zealand-dataand-information-management-principles/ 
Noordegraaf, M. (2016). Reconfiguring professional work: Changing forms of professionalism in public services. Administration \& Society, 48(7), 783-810. https://doi.org/10.1177/0095399713509242

Noordegraaf, M., \& Stewart, R. (2000). Managerial behaviour research in private and public sectors: distinctiveness, disputes and directions. Journal of Management Studies, $37(3), 427-443$.

Official Information Act (1982). Retrieved from http://www.legislation.govt.nz/

Oliver, G. (2005). Information in organisations: a comparative study of information cultures ( $\mathrm{PhD}$ Thesis). Monash University, School of Information Management \& Systems, Melbourne, Australia.

Oliver, G. (2008). Information culture: exploration of differing values and attitudes to information in organisations. Journal of Documentation, 64(3), 363-385.

Oliver, G. (2011). Organisational culture for information managers. Chandos Publishing.

Oliver, G., Evans, J., Reed, B., \& Upward, F. (2009). Achieving the right balance: Recordkeeping informatics, Part 1. IQ: The RIM Quarterly, 25(4), 18-21.

Oliver, G., Evans, J., Reed, B., \& Upward, F. (2010). Achieving the right balance: Recordkeeping informatics, Part 2. IQ: The RIM Quarterly, 26(1), 42-45.

Oliver, G., \& Foscarini, F. (2014). Records management and information culture: tackling the people problem.

Oliver, G., Foscarini, F., Sinclair, C., Nicholls, C., \& Loriente, L. (2018). Ethnographic sensitivity and current recordkeeping: Applying information culture analysis in the workplace. Records Management Journal, 28(2), 175-186. https://doi.org/10.1108/RMJ-08-2017-0021

Omar, K., Stockdale, R., \& Scheepers, H. (2014). Social media use in local government: An Australian perspective. International Journal of Public Administration, 37(10), 666675. https://doi.org/10.1080/01900692.2014.903270

O’Meara, E., \& Tuomala, M. (2012). Finding balance between archival principles and reallife practices in an institutional repository. Archivaria, Spring(73), 81-103. 
O’Neill, R. (2009). E-government: transformation of public governance in New Zealand? (PhD Thesis). Victoria University of Wellington, School of Government, Wellington, New Zealand.

Organisation for Economic Co-operation and Development. (2014). Working smarter in tax debt management. Paris, France: OECD Publishing. Retrieved from http://dx.doi.org/10.1787/9789264223257-en

Orlikowski, W. J. (2006). Material knowing: the scaffolding of human knowledgeability. European Journal of Information Systems, 15, 460-466.

Orlikowski, W. J. (2007). Sociomaterial practices: exploring technology at work. Organization Studies, 28, 1435-1448.

Orlikowski, W. J., \& Baroudi, J. J. (1991). Studying information technology in organizations: research approaches and assumptions. Information Systems Research, 2(1), 1-28.

Owens, I., Wilson, T. D., \& Abell, A. (1995). Information and business performance: a study of information systems and services in high-performing companies. Information Research, 1(1). Retrieved from http://informationr.net/ir/1-2/paper5.html

Pare, F. X. (2011). Personal information management among office support staff in a university environment: An exploratory study ( $\mathrm{PhD}$ Thesis). McGill University, Montreal.

Pors, A. S. (2015). Becoming digital - passages to service in the digitized bureaucracy. Journal of Organizational Ethnography, 4(2), 177-192. https://doi.org/10.1108/JOE08-2014-0031

Privacy Act (1993). Retrieved from http://legislation.govt.nz

Public Records Act, Pub. L. No. 40 (2005). Retrieved from http://www.legislation.govt.nz

Reddick, C. G., Abdelsalam, H. M., \& Elkadi, H. (2011). The influence of e-government on administrative discreation: The case of local governments in Egypt. Public Administration and Development, 31(5), 390-407. https://doi.org/10.1002/pad.615

Reed, B. (2007). Reading the records continuum. Archives and Manuscripts, 33(1), 18-43.

Rhodes, R. A. W. (2007). Understanding governance: ten years on. Organization Studies, 28(8), 1243-1264. 
Ridley, D. (2008). The literature review: a step by step guide for students. London: Sage Publications.

Robbins, S. P., \& Judge, T. (2008). Essentials of organizational behavior (9th ed). Upper Saddle River, N.J: Pearson/Prentice Hall.

Rohr, J. A. (1988). Bureaucratic morality in the United States. International Political Science Review, 9(3), 167-178.

Schuppan, T. (2015). Service workers on the electronic leash? Street-level bureaucrats in emerging information and communication technology work contexts. In P. Hupe, M. Hill, \& A. Buffat (Eds.), Understanding street-level bureaucracy (pp. 243-260). Bristol, England: Policy Press.

Scott, P. G. (1997). Assessing determinants of bureaucratic discretion: an experiment in street-level decision making. Journal of Public Administration Research and Theory, $35(23)$.

Shankar, K. (2004). Recordkeeping in the production of scientific knowledge: an ethnographic study. Archival Science, 4(3-4), 367-382.

Sharman, J. C. (2009). Privacy as roguery: Personal financial information in an age of transparency. Public Administration, 87(4), 717-731. https://doi.org/10.1111/j.14679299.2009.01785.x

Shaw, R., \& Eichbaum, C. (2008). Public policy in New Zealand (Vol. 2). North Shore: Pearson Education.

Shaw, S. G., Pedersen, S., Cooley, D., \& Callingham, R. (2013). Intentions and behaviours: Record-keeping practices of pre-service teachers during professional experience. Australian Journal of Teacher Education, 38(6), 71-87. https://doi.org/10.14221/ajte.2013v38n6.3

Simon, H. A. (1976). Administrative behavior : a study of decision-making processes in administrative organization (Third Edition). New York: Free Press.

Snellen, I. (1998). Street level bureaucracy in an information age. In I. Snellen, W. B. H. J. van de Donk, \& J. P. Baquiast (Eds.), Public Administration in an Information Age: A Handbook (pp. 497-504). Amsterdam, Netherlands: IOS Press. 
Spinuzzi, C. (2003). Tracing genres through organizations: a sociocultural approach to information design. Cambridge, Mass: MIT Press.

Stake, R. E. (2005). Qualitative Case Studies. In N. K. Denzin \& Y. S. Lincoln (Eds.), The Sage Handbook of Qualitative Research (Vol. Third, pp. 443-466). Thousand Oaks: Sage Publications.

State Services Commission. (2011). Human resource capability (HRC) survey of public service departments as at 30 June 2011. Wellington, New Zealand: State Services Commission. Retrieved from http://www.ssc.govt.nz/sites/all/files/hrc-survey2011.pdf

State Services Commission. (2014). Human resource capability (HRC) survey (workforce statistics). Wellington, New Zealand: State Services Commission. Retrieved from http://www.ssc.govt.nz/workforce-stats

State Services Commission. (2015). Human resource capability (HRC) in the New Zealand state sector 2015. Wellington, New Zealand: State Services Commission. Retrieved from https://www.ssc.govt.nz/sites/all/files/HRCReport-2015.pdf

State Services Commission. (2017). Standards of Integrity and Conduct. Retrieved 25 May 2017, from http://www.ssc.govt.nz/appendix-1-standards-integrity-and-conduct-stateservices

Statistics Act (1975). Retrieved from http://www.legislation.govt.nz/

Stevenson, A., \& Callaghan, S. (2008). Digitisation and matauranga Māori. Wellington, New Zealand: Victoria University of Wellington. Retrieved from http://hdl.handle.net/10063/608

Strong, D., Volkoff, O., Johnson, S., Pelletier, L., Tulu, B., Bar-On, I., ... Garber, L. (2014). A theory of organization-EHR affordance actualization. Journal of the Association for Information Systems, 15(2), 53-85. https://doi.org/10.17705/1jais.00353

Sundqvist, A., \& Svärd, P. (2016). Information culture and records management: a suitable match? Conceptualizations of information culture and their application on records management. International Journal of Information Management, 36(1), 9-15. https://doi.org/10.1016/j.ijinfomgt.2015.08.004 
Svärd, P. (2014). The impact of information culture on information/records management: A case study of a municipality in Belgium. Records Management Journal, 24(1), 5-21. https://doi.org/10.1108/RMJ-04-2013-0007

Tax Administration Act 1994, 1994 No 166 § (1994). Retrieved from http://www.legislation.govt.nz/act/public/1994/0166/latest/DLM348343.html?search= ta_act_T_ac\%40ainf $\% 40$ anif_an $\% 40 \mathrm{bn} \% 40 \mathrm{rn} \_25 \_\mathrm{a} \& \mathrm{p}=3$

Te Puni Kōkiri. (2011). Te Puni Kōkiri Statement of Corporate Intent 2011-2014 (Statement of Intent) (p. 50). Wellington, New Zealand: Te Puni Kōkiri. Retrieved from http://www.tpk.govt.nz/en/in-print/our-publications/corporate-documents/statementof-intent-2011-2014/download/tpk-soi-2011-en.pdf

Te Puni Kōkiri. (2014). Te puni hononga ā rohe - regional partnerships job description. Te Puni Kōkiri. Retrieved from http://www.tpk.govt.nz/docs/vacancies/AdvisorRegional-Job-Description.pdf

Te Puni Kōkiri. (2016). DA646: Appraisal report. Wellington, New Zealand: Te Puni Kōkiri. Retrieved from https://www.archway.archives.govt.nz/ViewEntity.do?code=DA646 Te Puni Kōkiri. (n.d.). LiveLink - 2. adding metadata to documents. Te Puni Kōkiri. The Law Commission. (2012). The public's right to know: Review of the official information legislation (No. NZLC R125). Wellington, New Zealand: The Law Commission. Retrieved from http://r125.publications.lawcom.govt.nz

Tope, D., Chamberlain, L. J., Crowley, M., \& Hodson, R. (2005). The benefits of being there: Evidence from the literature on work. Journal of Contemporary Ethnography, 34(4), 470-493. https://doi.org/10.1177/0891241605276692

Tough, A. (2006). Records and the transition to the digital. In A. Tough \& M. Moss (Eds.), Record Keeping in a Hybrid Environment: Managing the Creation, Use, Preservation and Disposal of Unpublished Information Objects in Context (pp. 1-25). Chandos Publishing.

Trace, C. B. (2002). What is recorded is never simply 'what happened': record keeping in modern organizational culture. Archival Science, 2(1-2), 137-159. 
Travica, B. (2008). Influence of information culture on adoption of a self-service system. Journal of Information, Information Technology, and Organizations, 3. Retrieved from

http://link.galegroup.com.helicon.vuw.ac.nz/apps/doc/A199195144/AONE?u=vuw\&s id $=$ AONE $\&$ xid $=8 \mathrm{a} 9593 \mathrm{e} 6$

Upward, F. (1996). Structuring the records continuum - part one: Postcustodial principles and properties. Archives and Manuscripts, 24(2), 268-285.

Upward, F. (2000). Modelling the continuum as paradigm shift in recordkeeping and archiving processes, and beyond - a personal reflections. Records Management Journal, 10(3), 115-139.

Upward, F. (2010). Request for permission to use the continuum diagram.

Upward, F., \& McKemmish, S. (2006). Teaching recordkeeping and archiving continuum style. Arch Sci, 6, 216-230.

Upward, F., Reed, B., Oliver, G., \& Evans, J. (2013). Recordkeeping informatics: Refiguring a discipline in crisis with a single minded approach. Records Management Journal, 23(1), 37-50.

Venkatesh, V., \& Bala, H. (2008). Technology acceptance model 3 and a research agenda on Interventions. Decision Sciences, 39(2), 273-315. https://doi.org/10.1111/j.15405915.2008.00192.x

Venkatesh, V., \& Davis, F. D. (2000). A theoretical extension of the technology acceptance model: four longitudinal field studies. Management Science, 46(2), 186-204.

Venkatesh, V., Morris, M. G., Davis, G. B., \& Davis, F. D. (2003). User acceptance of information technology: toward a unified view. MIS Quarterly, 27(3), 425-478.

Vinzant, J., \& Crothers, L. (1996). Street-level leadership: rethinking the role of public servants in contemporary governance. The American Review of Public Administration, 26, 457-475.

Viseu, A., Clement, A., \& Aspinall, J. (2004). Situating privacy online: complex perceptions and everyday practices. Information, Communication \& Society, 7(1), 92-114. 
Walsham, G. (1995). Interpretive case studies in IS research: Nature and method. European Journal of Information Systems, 4(2), 74-81. https://doi.org/doi:10.1057/ejis.1995.9

Wareham, E. (2002). From explorers to evangelists: archivists, recordkeeping, and remembering in the Pacific Islands. Archival Science, 2(3), 187-207.

White, N. (2007). Free and frank: Making the Official Information Act 1982 work better. Wellington: Victoria University of Wellington.

Whittaker, S., \& Sidner, C. (1996). E-mail overload: exploring personal information management of e-mail (pp. 276-283). Presented at the Conference on Human Factors in Computing Systems (CHI '96), Vancouver, Canada.

Widén-Wulff, G. (2000). Business information culture: a qualitative study of the information culture in the Finnish insurance business. Information Research, 5(3). Retrieved from http://informationr.net/ir/5-3/paper77.html

Willis, A. (2005). Corporate governance and management of information and records. Records Management Journal, 15(2), 86-97. https://doi.org/10.1108/09565690510614238

Winget, M. A., Chang, K., \& Tibbo, H. (2007). Personal email management on the university digital desktop: User behaviors vs. archival best practices. Proceedings of the American Society for Information Science and Technology, 43, 1-13. https://doi.org/10.1002/meet.14504301127

Wright, K. (2014). Broadening the record and expanding the archives. Archives and Manuscripts, 42(2), 219-221. https://doi.org/10.1080/01576895.2014.911693

Wright, T. (2013). Information culture in a government organization: Examining records management training and self-perceived competencies in compliance with a records management program. Records Management Journal, 23(1), 14-36. https://doi.org/10.1108/09565691311325004

Yakel, E. (1997). Recordkeeping in radiology: the relationship between activities and records in radiological processes ( $\mathrm{PhD}$ Thesis). The University of Michigan, Ann Arbor. 
Yakel, E. (2001). The social construction of accountability: radiologists and their recordkeeping practices. The Information Society, 17(4), 233-245.

Yin, R. K. (2009). Case study research: design and methods. Thousand Oaks: Sage Publications.

Yin, R. K. (2014). Case study research: Design and methods (5th ed.). Los Angeles, CA: Sage Publications.

Zhang, J. (2012). Original order in digital archives. Archivaria, Fall(74), 167-193.

Zuboff, S. (1988). In the age of the smart machine. New York, NY: BasicBooks. 